\title{
Microvascular and blood-brain barrier dysfunction in Alzheimer's disease
}

Citation for published version (APA):

van de Haar, H. J. (2016). Microvascular and blood-brain barrier dysfunction in Alzheimer's disease: New insights from quantitative magnetic resonance imaging. [Doctoral Thesis, Maastricht University]. Maastricht University. https://doi.org/10.26481/dis.20161110hh

Document status and date:

Published: 01/01/2016

DOI:

10.26481/dis.20161110hh

Document Version:

Publisher's PDF, also known as Version of record

\section{Please check the document version of this publication:}

- A submitted manuscript is the version of the article upon submission and before peer-review. There can be important differences between the submitted version and the official published version of record.

People interested in the research are advised to contact the author for the final version of the publication, or visit the DOI to the publisher's website.

- The final author version and the galley proof are versions of the publication after peer review.

- The final published version features the final layout of the paper including the volume, issue and page numbers.

Link to publication

\footnotetext{
General rights rights.

- You may freely distribute the URL identifying the publication in the public portal. please follow below link for the End User Agreement:

www.umlib.nl/taverne-license

Take down policy

If you believe that this document breaches copyright please contact us at:

repository@maastrichtuniversity.nl

providing details and we will investigate your claim.
}

Copyright and moral rights for the publications made accessible in the public portal are retained by the authors and/or other copyright owners and it is a condition of accessing publications that users recognise and abide by the legal requirements associated with these

- Users may download and print one copy of any publication from the public portal for the purpose of private study or research.

- You may not further distribute the material or use it for any profit-making activity or commercial gain

If the publication is distributed under the terms of Article $25 \mathrm{fa}$ of the Dutch Copyright Act, indicated by the "Taverne" license above, 


\section{Microvascular and blood-brain barrier dysfunction in Alzheimer's disease}

New insights from

quantitative magnetic resonance imaging 
(c) Harm van de Haar, Maastricht 2016

All rights reserved. No part of this book may be reproduced or transmitted in any form or by any means, without prior permission in writing by the author, or when appropriate, by the publishers of the publications.

Layout and Cover: Harm van de Haar

Printing: Ipskamp Printing B.V., Enschede 


\title{
Microvascular and blood-brain barrier dysfunction in Alzheimer's disease
}

\author{
New insights from \\ quantitative magnetic resonance imaging
}

\section{PROEFSCHRIFT}

ter verkrijging van de graad van doctor aan de Universiteit Maastricht, op gezag van de Rector Magnificus, Prof. dr. Rianne M. Letschert, volgens het besluit van het College van Decanen, in het openbaar te verdedigen

op donderdag 10 november 2016 om 12.00 uur

door

Harmen Jan van de Haar

Geboren 21 januari, 1987, te Ede 


\section{Promotores}

Prof. Dr. Ir. W.H. Backes

Prof. Dr. F.R.J. Verhey

\section{Copromotores}

Dr. J.F.A. Jansen

Dr. Ir. M.J.P. van Osch

\section{Beoordelingscommissie}

Prof. Dr. J.E. Wildberger (voorzitter)

Dr. M.P.J. van Boxtel

Dr. J.A.H.R. Claassen (Radboud Universitair Medisch Centrum)

Prof. Dr. R.J. van Oostenbrugge

Prof. Dr. H.E. de Vries (Vrije Universiteit Medisch Centrum)

The printing of this thesis was financially supported by Bayer Healthcare.

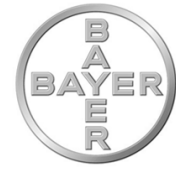




\section{Table of Contents}

\section{Chapter 1}

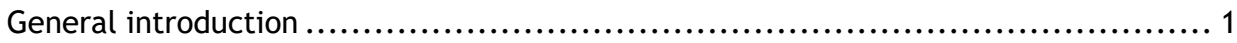

Chapter 2 - Neuroscience and Biobehavioral Reviews (2015)

Blood-brain barrier impairment in dementia: current and future in vivo assessments .13

Chapter 3 - Radiology (2016)

Blood-brain barrier leakage in early Alzheimer's disease

\section{Chapter 4 - Submitted for publication}

Subtle blood-brain barrier leakage rate and spatial extent:

considerations for dynamic contrast-enhanced MRI

Chapter 5 - Neurobiology of Aging (2016)

Neurovascular unit impairment in early Alzheimer's disease measured with MRI

Chapter 6 - Submitted for publication

The effect of white matter hyperintensities on hippocampal structural connectivity

in Alzheimer's disease

\section{Chapter 7}

General discussion

Summary 148

Samenvatting

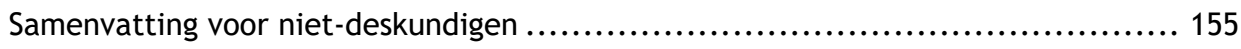

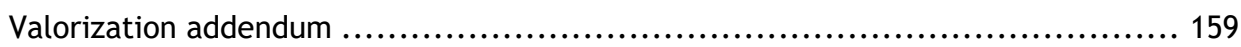

Dankwoord ................................................................. 163

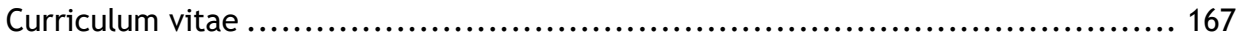

List of publications ...................................................... 169 



\section{Chapter 1}

General introduction 


\section{Alzheimer's disease}

At the $37^{\text {th }}$ Meeting of the Society of Southern German Psychiatrists in November 1906, dr. Alois Alzheimer described a series of pathological changes in the brain of a patient who was suffering from dementia ${ }^{1}$. He described a disease that is now known as "Alzheimer's Disease" (AD). AD is the leading cause of cognitive decline and dementia in the elderly, and has a severe impact on the lives of millions ${ }^{2}$. The slow cognitive deterioration coupled with the insidious onset affects not only the patient, but also their loved ones ${ }^{3}$. But besides the cognitive decline, dr. Alzheimer also described several histopathological changes to the brain of his patient, including neuritic plaques and neurofibrillary tangles, which are now still recognized as hallmark symptoms. However, over 100 years of research have not provided conclusive evidence of the etiology of $A D$, nor is a cure currently available.

\section{The pathophysiology of $A D$}

The research into the underlying pathology of $A D$ has mostly focused on the plaques and tangles, which are abnormal accumulations of insoluble proteins amyloid $B$ and tau, typically present in $\mathrm{AD}^{4}$. The popular "amyloid hypothesis" states that these proteins are key to the pathogenesis of $A D^{5}$. According to this hypothesis, an imbalance of production versus clearance of amyloid $B$ causes accumulation of this protein, which is thought to have toxic effects ${ }^{6}$. Subsequently occurring complex molecular and cellular processes are thought to lead to neurodegeneration, atrophy and gliosis, causing cognitive decline and dementia ${ }^{7}$. Although the exact processes, including the role of the tau protein, are still unclear, research into the amyloid hypothesis has provided substantial evidence for an association of these proteins with $A D$. The amyloid hypothesis has also provided biomarkers for $A D$, which are means to detect $A D$ before the symptoms are fully expressed. Biomarkers for $A D$ include an elevated relative amyloid $B$ concentration in the cerebrospinal fluid and hippocampal volume loss on structural Magnetic Resonance Imaging (MRI) scans ${ }^{3,8}$. It has also allowed for the generation of a functional biomarker model of $A D$, which helps to stage the disease and possibly to examine the effects of potential drugs on different aspects of the disease (Figure 1.1) ${ }^{7}$. However, given the enormous current and expected future impact of dementia, there is an urgent need for means that either cure or delay the disease process. To find such means, early diagnosis of $A D$ is very important, as early detection of the disease process allows for investigation of the processes causing cognitive decline and dementia and earlier intervention. Therefore, new and more accurate biomarkers are needed. 
Unfortunately, the amyloid hypothesis has so far not been able to provide either a cure, or an reliable, early biomarker.

The amyloid hypothesis does not include vascular components in the description of the disease evolution, and (cerebro)vascular pathology is even an exclusion criterion for $\mathrm{AD}^{3}$. However, more recent evidence suggests that patients diagnosed with $A D$ often have mixed pathology, exhibiting both classical AD symptoms and vascular pathology ${ }^{9}$. Furthermore, vascular pathology also exacerbates cognitive decline in AD patients (Figure 1.2). This, combined with evidence showing that amyloid deposition is not specific for $A D$, has given rise to new hypotheses regarding the pathophysiology occurring in $A D$ patients ${ }^{10}$. Increasing evidence supports the vascular hypothesis, which states that cerebrovascular pathology plays a key role, and that amyloid $B$ is not the cause of $A D$, but a part of a much larger pathological cascade leading to dementia ${ }^{11}$. 


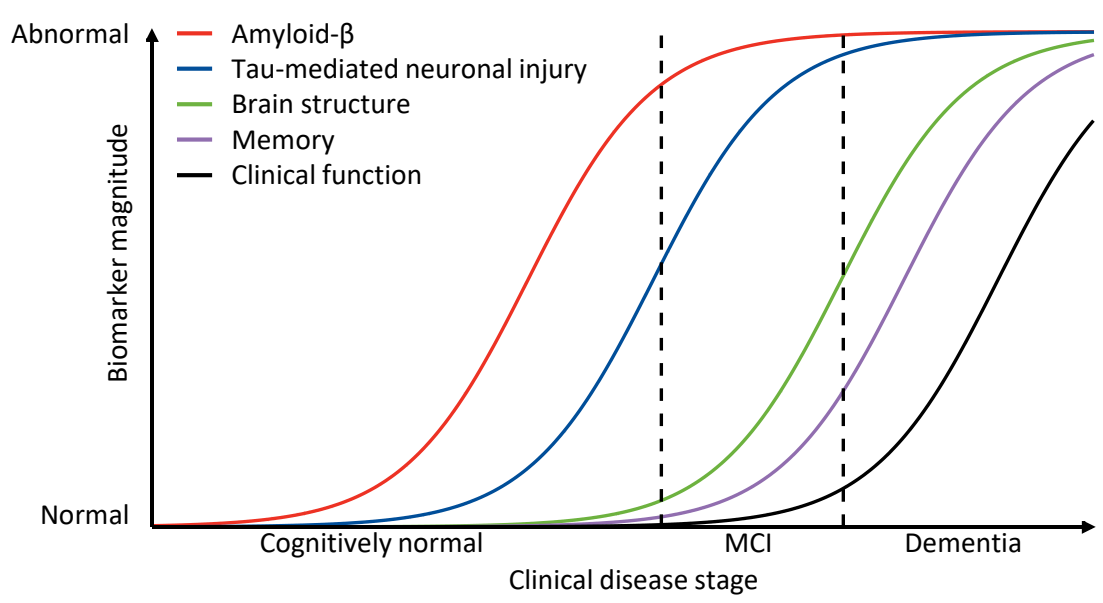

Figure 1.1: Hypothetical biomarker model for the different stages of Alzheimer's disease ${ }^{7}$. Note that the progression of cerebrovascular pathology is still unknown, and therefore not included in this model.

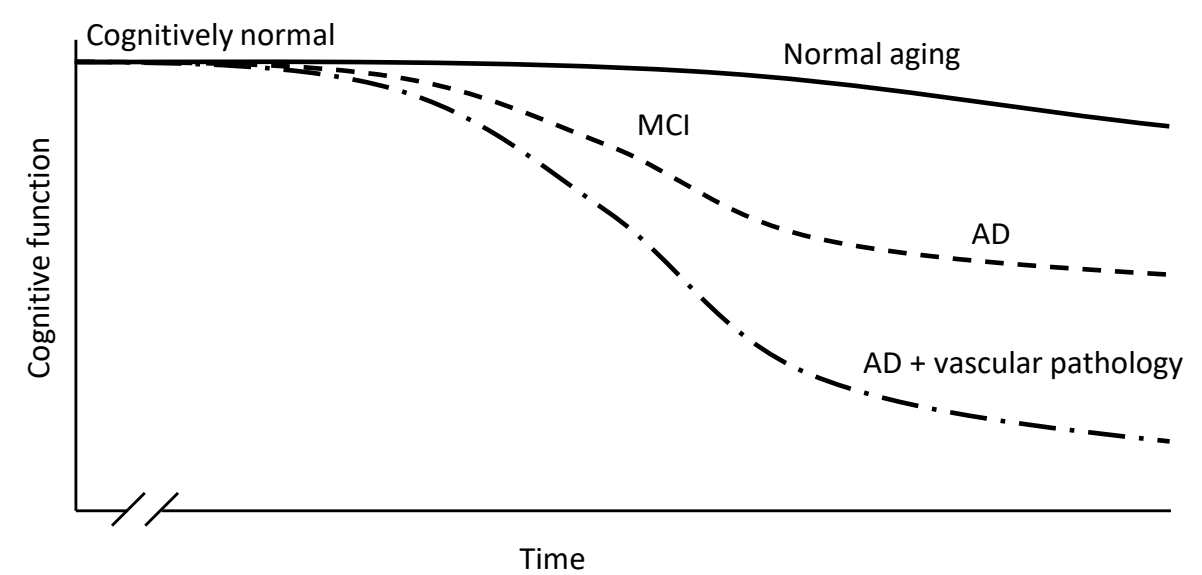

Figure 1.2: Hypothetical model of cognitive function during normal aging and AD. A combination of $A D$ with (cerebro)vascular pathology increases the rate of cognitive decline. The graph is based on multiple sources ${ }^{25-28}$. 
The vascular hypothesis for $A D$ states that vascular risk factors, such as hypertension and atherosclerosis, start a cascade of microvascular events which interact with $A D$ pathology ${ }^{10,12}$. An overview of the difference between the pathways of the amyloid and vascular hypotheses is shown in Figure 1.3. The vascular dysfunction occurs mainly at the microvascular level, where the blood vessels are strictly regulated by the so-called neurovascular unit ${ }^{13}$. The neurovascular unit is the collection of cellular and subcellular structures that work together to maintain homeostasis and support and protect the neurons and neuronal environment. An important part of the neurovascular unit is the blood-brain barrier (BBB), which prevents neurotoxic substances circulating in the blood from exiting the blood space while allowing nutrients to pass and to support the energy demand of the neurons. The neurovascular unit also regulates blood flow according to local demand and plays a role in the cerebral immune system. There are several findings that point towards involvement of vascular pathology in $A D^{14}$. For example, cerebrovascular pathology, including microbleeds, infarctions and lacunes, in people with dementia is quite common ${ }^{10,15,16}$. Also, it is very common in AD to find White Matter Hyperintensities (WMHs) on MRI images, which are thought to be ischemic in nature, and caused by vascular damage ${ }^{17-19}$. WMHs are especially of interest as they increase the risk to develop $A D$ and are associated with an increased rate of cognitive decline $e^{20,21}$. 


\section{Amyloid hypothesis}

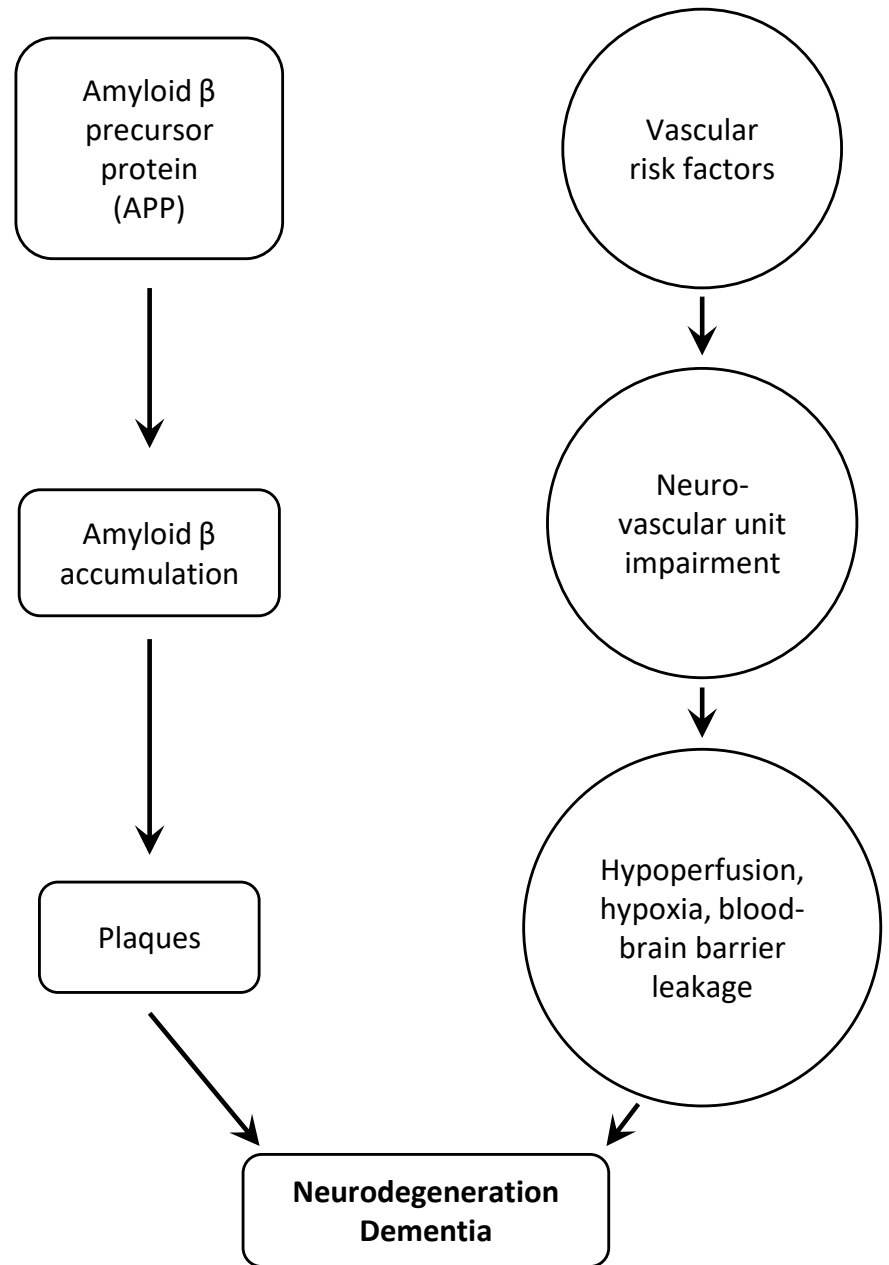

Vascular hypothesis 


\section{Neuroimaging}

Diagnosis of $A D$ can be difficult, and neuroimaging can help with this as it is used to exclude other potential causes of the cognitive impairment, such as a brain tumor or a subdural hematoma. It can also be used to provide further proof for $A D$, by measuring hippocampal atrophy using MRI or the distribution of amyloid $B$ using Positron Emission Tomography (PET) combined with an amyloid tracer ${ }^{22}$. However, hippocampal atrophy is a late marker, often observed quite some time after initial onset of the disease, when the cognitive impairment has already started (Figure 1.1). A disadvantage of PET is the necessity of using a radioactive tracer, and, combined with the fact that amyloid deposition also occurs in cognitively healthy subjects and in other diseases than AD, amyloid imaging may not be the most optimal choice for a biomarker ${ }^{23}$.

MRI is commonly used in $A D$ to detect atrophy and exclude other causes for the dementia. It also offers several promising techniques which may serve to further investigate different possible features of the vascular hypothesis.

\section{DCE-MRI}

Impairment of the BBB and the local blood volume can be measured using Dynamic Contrast-Enhanced (DCE) MRI. By injecting a (gadolinium-based) contrast agent while continuously acquiring images, the distribution of the contrast agent can be measured over time. Because a healthy BBB prevents the contrast agent from leaving the blood vessels, there should be no extravascular contrast agent. Therefore, the presence of contrast agent outside the blood pool can be considered proof of an impaired BBB. DCEMRI cannot only be used to diagnose an impaired $B B B$, it can also provide quantitative measures of the leakiness of the BBB.

\section{ASL}

Local cerebral blood flow can be measured using Arterial Spin Labeling (ASL). This technique requires no exogenous contrast agent, but instead uses magnetically labeled water (protons) in the arterial blood as an endogenous "contrast agent". By mapping the accumulation of labeled water in brain tissue, the spatiotemporal distribution of the blood flow can be established. By analyzing the difference between images with labeled protons and images without labeling (the control images), local cerebral blood flow can be quantified. Because the blood flow is tightly regulated by the neurovascular unit, local blood flow differences may indicate neurovascular unit impairment by proxy. 


\section{Diffusion MRI}

Diffusion MRI is a technique that allows for measurement of proton movement due to the application of diffusion-weighting gradients into the MRI sequence. The brain white matter is composed of axons, which connect different parts of the cortex. This organization changes the diffusion of protons from being completely random, as in a reservoir of water, into an anisotropic process due to restricted diffusion in directions perpendicular to the white matter fibers. This allows for assessment of the integrity of the white matter, and the reconstruction of the geometry of white matter bundles. Since WMHs are associated with demyelination, it is likely that they affect these white matter tracts, which may be studied using diffusion $\mathrm{MRI}^{24}$.

\section{Aim and outline of this thesis}

By expanding our knowledge of the underlying pathophysiology of $A D$, new biomarkers and new targets for treatments may be found. As vascular pathology is hypothesized to be an important factor in the pathophysiology of $A D, M R I$ can be considered a promising imaging modality, since it can be used to measure different aspects of the vascular integrity in the brain of living humans. Technological advances have enabled the use of neuroimaging techniques to measure the integrity of the $\mathrm{BBB}$, which constitutes an essential part of the neurovascular unit. Because it can be measured in vivo, there is no need to perform the investigations after death or to obtain post-mortem tissue. As AD is a slowly progressing degenerative disease, death usually occurs when the disease has progressed to an advanced stadium, which makes it difficult to study the disease at an earlier stage. Modern neuroimaging methods overcome this limitation, opening up new lines of research. Therefore, the first research questions of this thesis are:

In what ways can the integrity of the BBB be non-invasively measured in vivo?

and

What techniques have already been used to measure BBB integrity in early $A D$ in vivo?

In Chapter 2, we have reviewed the literature on available technologies that enable the in vivo assessment of the condition of the BBB. The review results showed that it is possible to examine the $B B B$ in vivo, but that it is not often performed in $A D$, which lead to the next question:

Is there BBB impairment in early $A D$ ? 
In Chapter 3, we discuss the findings of measuring BBB impairment by applying a dedicated DCE-MRI sequence in patients with early $A D$ and healthy controls. Because WMHs are indicative of vascular damage, the locations which most often have WMHs offer the best chance at finding BBB impairment. Therefore, the field-of-view of the DCE-MRI sequence was positioned to provide optimal coverage of the area around the lateral ventricles, which is where the WMHs are most often encountered.

We found that using DCE-MRI to measure the subtle BBB leakage of early AD is possible but difficult. This leads to the question:

How can the sensitivity of a DCE-MRI experiment be improved to detect subtle BBB leakage?

In Chapter 4, we investigated this by combining the DCE-MRI data with computer simulation experiments, to analyze the influence of several methodological alterations on the ability to detect BBB leakage.

Besides the BBB, other aspects of the neurovascular unit may also be impaired in early $A D$, such as the local blood flow, which raises the questions:

Is the local blood perfusion in early $A D$ altered?

and

Is there a correlation in early $A D$ between blood flow impairment and other functional elements of the neurovascular unit?

Chapter 5 describes the measurement of the local cerebral blood flow using an ASL sequence. Because the DCE-MRI and ASL sequences were used in the same subjects, we also analyzed the correlation between BBB impairment and perfusion differences.

Besides vascular pathology, WMHs are also associated with demyelination. This indicates that WMHs also disrupt the normal connectivity patterns of the brain, which may also contribute to the cognitive decline. Therefore, the last main question of this thesis is:

What is the impact of WMHs on the brain white matter in terms of structural connectivity?

Chapter 6 describes the result of using diffusion MRI to reconstruct the brain white matter tracts and investigate the influence of WMHs on the volume of these tracts. Since 
the hippocampus plays a major role in the pathophysiology of $A D$, this chapter focuses on the white matter tracts connecting the hippocampi with the rest of the cerebrum.

In Chapter 7, we present our conclusions and the answers to the above questions. Furthermore, the results of the different studies are combined and discussed in a broader perspective. 


\section{References}

1 Alzheimer A. Uber eine eigenartige Erkrankung der Hirnrinde. Allg Zeitschrife Psychiatr 1907; 64: 146-148.

Alzheimer's Association. 2015 Alzheimer's disease facts and figures. Alzheimers Dement 2015; 11: 332-84.

McKhann GM, Knopman DS, Chertkow H, Hyman BT, Jack CR, Kawas CH et al. The diagnosis of dementia due to Alzheimer's disease: Recommendations from the National Institute on AgingAlzheimer's Association workgroups on diagnostic guidelines for Alzheimer's disease. Alzheimer's Dement 2011; 7: 263-269.

Hardy J. The amyloid hypothesis for Alzheimer's disease: A critical reappraisal. J Neurochem 2009; 110: 1129-1134.

Selkoe DJ. The Molecular Pathology of Alzheimer's Disease. Neuron 1991; 6: 487-498.

Selkoe DJ. Alzheimer's disease results from the cerebral accumulation and cytotoxicity of amyloid beta-protein. J Alzheimers Dis 2001; 3: 75-80.

Jack CR, Knopman DS, Jagust WJ, Shaw LM, Aisen PS, Weiner MW et al. Hypothetical model of dynamic biomarkers of the Alzheimer's pathological cascade. Lancet Neurol 2010; 9: 119-128.

Handels RL, Aalten P, Wolfs CA, OldeRikkert M, Scheltens P, Visser P et al. Diagnostic and economic evaluation of new biomarkers for Alzheimer's disease: the research protocol of a prospective cohort study. BMC Neurol 2012; 12: 72.

Echávarri C, Burgmans S, Caballero MC, García-Bragado F, Verhey FRJ, Uylings HBM. Co-occurrence of different pathologies in dementia: Implications for dementia diagnosis. J Alzheimer's Dis 2012; 30 : 909-917.

10 De La Torre JC. Is Alzheimer's disease a neurodegenerative or a vascular disorder? Data, dogma, and dialectics. Lancet Neurol 2004; 3: 184-190.

11 Drachman DA. The amyloid hypothesis, time to move on: Amyloid is the downstream result, not cause, of Alzheimer's disease. Alzheimer's Dement 2014; 10: 372-380.

12 Zlokovic BV. Neurovascular mechanisms of Alzheimer's neurodegeneration. Trends Neurosci 2005; 28: 202-208.

Stanimirovic DB, Friedman A. Pathophysiology of the neurovascular unit: disease cause or consequence? J Cereb Blood Flow Metab 2012; 32: 1207-1221.

14 Benarroch EE. Neurovascular unit dysfunction: A vascular component of Alzheimer disease? Neurology. 2008; 70: 1941.

Gorelick PB, Scuteri A, Black SE, DeCarli C, Greenberg SM, ladecola C et al. Vascular Contributions to Cognitive Impairment and Dementia: A Statement for Healthcare Professionals From the American Heart Association/American Stroke Association. Stroke 2011; 42: 2672-2713.

Schneider JA, Arvanitakis Z, Bang W, Bennett DA. Mixed brain pathologies account for most dementia cases in community-dwelling older persons. Neurology 2007; 69: 2197-2204.

Shim Y, Morris J, Cairns N, Benzinger T, Xiong C. Pathological correlates of white matter hyperintensities on magnetic resonance imaging. Alzheimer's Dement 2011; 7: S706. hyperintensities and normal-appearing white matter integrity in the aging brain. Neurobiol Aging 2015; 36: 909-918. 
Wardlaw JM, Valdés Hernández MC, Muñoz-Maniega S. What are white matter hyperintensities made of? Relevance to vascular cognitive impairment. J Am Heart Assoc 2015; 4: 001140.

Frisoni GB, Galluzzi S, Pantoni L, Filippi M. The effect of white matter lesions on cognition in the elderly--small but detectable. Nat Clin Pract Neurol 2007; 3: 620-7.

Defrancesco M, Marksteiner J, Deisenhammer E, Kemmler G, Djurdjevic T, Schocke M. Impact of white matter lesions and cognitive deficits on conversion from mild cognitive impairment to Alzheimer's disease. J Alzheimers Dis 2013; 34: 665-72.

Ashraf A, Mehta P, Edison P. Amyloid imaging in dementia. Rev Clin Gerontol 2012; 23: 43-60.

Eisenmenger LB, Huo EJ, Hoffman JM, Minoshima S, Matesan MC, Lewis DH et al. Advances in PET Imaging of Degenerative, Cerebrovascular, and Traumatic Causes of Dementia. Semin Nucl Med 2016; 46: 57-87.

Englund E. Neuropathology of White Matter Changes in Alzheimer's Disease. Dement Geriatr Cogn Disord 1998; 9: 6-12.

Kalaria RN. The role of cerebral ischemia in Alzheimer's disease. Neurobiol Aging 2000; 21: 321-330.

Ferreira LK, Tamashiro-Duran JH, Squarzoni P, Duran FL, Alves TC, Buchpiguel C a et al. The link between cardiovascular risk, Alzheimer's disease, and mild cognitive impairment: support from recent functional neuroimaging studies. Rev Bras Psiquiatr 2014; 36: 344-357.

Roberts RO, Geda YE, Knopman DS, Cha RH, Pankratz VS, Boeve BF et al. Cardiac disease associated with increased risk of nonamnestic cognitive impairment. JAMA Neurol 2013; 70: 374-382.

Musicco M, Palmer K, Salamone G, Lupo F, Perri R, Mosti S et al. Predictors of progression of cognitive decline in Alzheimer's disease: The role of vascular and sociodemographic factors. J Neurol 2009; 256: 1288-1295. 


\section{Chapter 2}

\section{Blood-brain barrier impairment}

in dementia: current and future in vivo assessments

Harm J van de Haar, Saartje Burgmans, Paul AM Hofman, Frans RJ Verhey, Jacobus FA Jansen, Walter H Backes

Neuroscience and Biobehavioral Reviews (2015). doi: 10.1016/j.neubiorev.2014.11.022 


\section{Abstract}

Increasing evidence indicates that blood-brain barrier (BBB) impairment may play a role in the pathophysiology of cognitive decline and dementia. In vivo imaging studies are needed to quantify and localize the BBB defects during life, contemplating the circulatory properties. We reviewed the literature for imaging studies investigating BBB impairment in patients suffering from dementia. After selection, 11 imaging studies were included, of which 6 used contrast-enhanced magnetic resonance imaging (MRI), 2 used contrast-enhanced computed tomography (CT), and 3 positron emission tomography (PET). Primarily the MRI studies hint at a subtle increasing permeability of the $\mathrm{BBB}$, particularly in patients already exhibiting cerebrovascular pathology. More elaborate studies are required to provide convincing evidence on BBB impairment in patients with various stages of dementia with and without obvious cerebrovascular pathology. In the future, dynamic contrast enhanced MRI techniques and transport specific imaging using PET may further detail the research on the molecular nature of BBB defects. 


\section{Introduction}

Dementia is characterized by progressive cognitive decline and has a severe impact on the ability for independent living. It is an umbrella term and can have several causes. Most investigations of the pathophysiology of dementia have focused on Alzheimer's disease $(A D)$ and in particular its two biological markers: the senile plaques, which are accumulations of insoluble amyloid $B(A B)$ protein, and the neurofibrillary tangles, which are composed of the tau protein ${ }^{1}$. However, after decades of research, the pathophysiology of Alzheimer's dementia is still unclear and no effective cure has been found. Moreover, there is increasing doubt as to whether further investigation of the amyloid hypothesis, which states that $A B$ is the main cause of Alzheimer's dementia, is the right way to move forward ${ }^{2-4}$. As a result, interest in other potential causes of dementia has increased over the last years.

Special attention has been paid to the impact of vascular pathology. Multiple common findings in dementia suggest the presence of a strong link between dementia and vascular pathology, including the high incidence of cerebral amyloid angiopathy ${ }^{5}$, the vascular origin of most of the risk factors ${ }^{2}$, and the notion that treatment of these risk factors decelerates cognitive decline ${ }^{2,6}$. Also, vascular abnormalities such as white matter lesions (of presumed vascular origin), microbleeds, and infarctions (see Figure 2.1), are commonly found in brains of demented patients ${ }^{2}$. However, the underlying

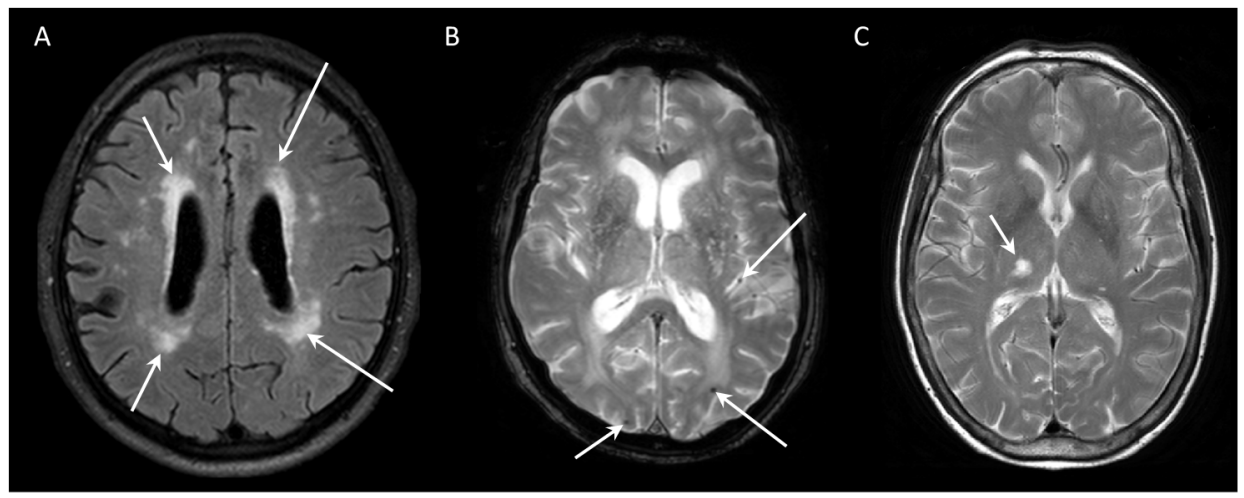

Figure 2.1: An overview of different types of cerebrovascular abnormalities commonly found in patients with dementia using MRI. A: a FLAIR image showing white matter lesions (arrows). B: a T2* weighted image showing microbleeds (arrows). C: A T2 weighted image showing a lacunar infarction (arrow). 
mechanism of such - often small vessel - pathologies and the link with dementia is not completely understood.

An important component of the cerebral microvasculature is the blood-brain barrier (BBB), which regulates the transport of molecules between the blood space and the parenchyma. Investigation of the cerebral microvasculature in AD has shown signs of BBB dysfunction. For example, there is evidence of vascular pathology involving the endothelial cells and the basement membrane, which are important components of the $\mathrm{BBB}^{7}$. Also, high levels of the albumin protein in the brain tissue were found which indicates BBB dysfunction ${ }^{8}$. Finally, the clearest example is the extravascular extracellular accumulation of $A B$, forming the senile plaques. $A B$ should be regulated by the $\mathrm{BBB}$ and accumulation probably indicates failure of the regulatory process. Therefore, the BBB in dementia is a promising target for research.

$A B$, and its relationship to Alzheimer's disease, has received a lot of attention over the years, including its interaction with the $\mathrm{BBB}^{9,10}$. However, there is also the possibility of other, still unknown, mechanisms through which BBB dysfunction may contribute to the pathogenesis of $A D$ and other forms of dementia. Insights into the pathologic mechanisms and role of the BBB in dementia require effective, preferably noninvasive, in vivo methods that are able to localize the $B B B$ impairment. This review will first give a brief overview of the BBB and literature on its general role in dementia. Subsequently, this review will evaluate the current in vivo imaging methods that can be used to investigate the $\mathrm{BBB}$, and will also discuss future promising methods.

\section{The Blood-Brain Barrier}

The main components of the BBB are specialized endothelial cells, which are supported by a basal membrane, pericytes and astrocytic endfeet. These constitute the capillary wall in the central nervous system (see Figure 2.2). The BBB prevents toxic compounds circulating in the blood from entering the central nervous system and removes waste products from the brain tissue, while allowing essential molecules to selectively pass. This maintains homeostasis and is accomplished through passive and active transport mechanisms (see Figure 2.3) ${ }^{11}$. The specialized endothelial cells are concatenated by tight junctions, and thus form a continuous layer at the luminal side of the capillaries. At the parenchymal side of the endothelium, pericytes and astrocytic endfeet are located, surrounded by a basement membrane. 


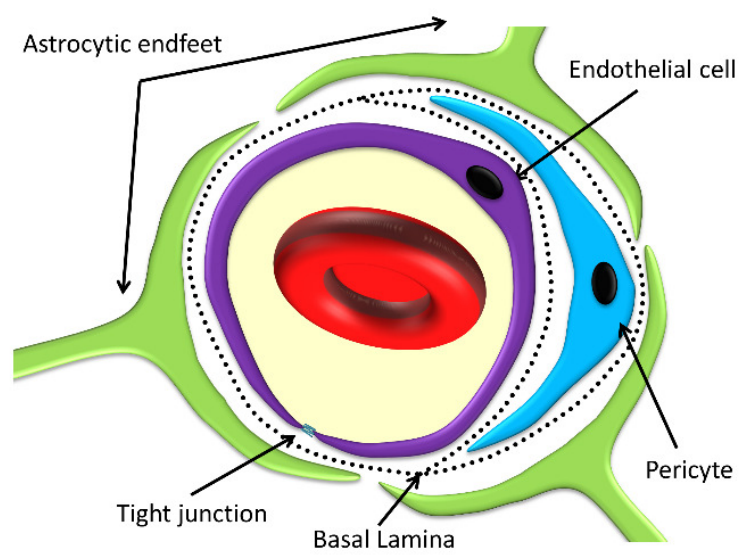

Figure 2.2: Schematic overview of the different components of the capillary neurovascular unit which houses the blood-brain barrier. The capillary wall is formed by endothelial cells, supported by pericytes and astrocytic endfeet. A basal lamina surrounds the endothelial cells and pericytes. Tight junctions close the gaps between endothelial cells, regulating transport through the gaps between the cells.

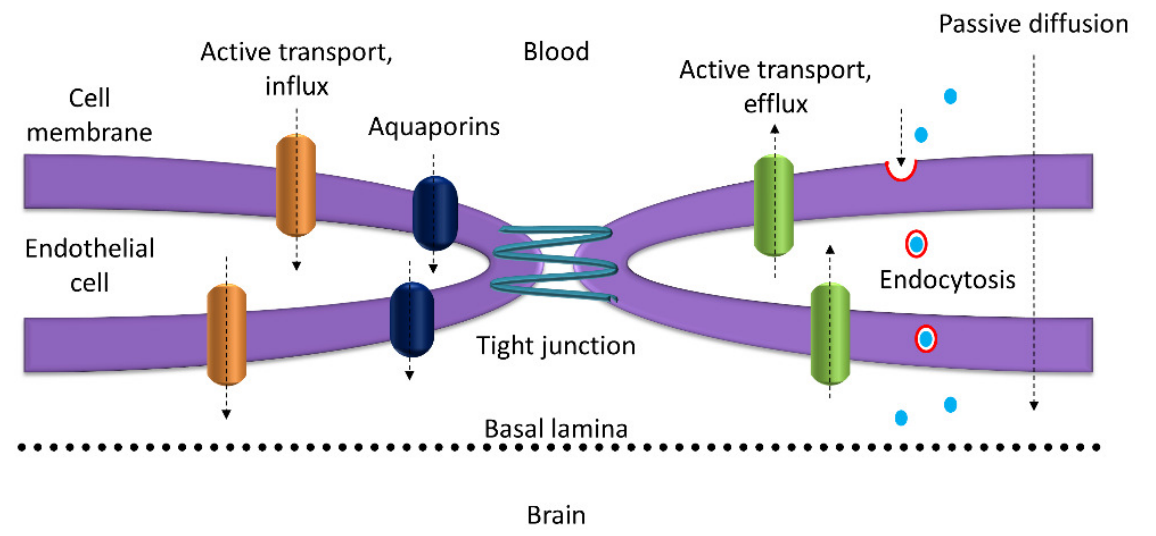

Figure 2.3: Schematic overview of different transport mechanisms through which compounds can cross the BBB. Small molecules such as $\mathrm{CO} 2$ and $\mathrm{O} 2$ cross the blood-brain barrier freely. Other compounds are strictly regulated via tight junctions, active transport pumps (efflux and influx), aquaporins and endocytosis (receptor-mediated and adsorptive) ${ }^{11}$. 
Due to its complex nature, various mechanisms may impair the function of the BBB. Loss of BBB function may cause damage to neuronal cells or worsen their condition, which in turn may lead to neurodegenerative disorders ${ }^{5}$. In the case of impaired tight junctions, the $\mathrm{BBB}$ becomes more permeable to compounds with increasing size and lipophilicity ${ }^{12,13}$. This is a passive process, which shall be referred to as BBB permeability or leakage in this review. Furthermore, it should be noted that the loss of tight junctions also reduces the polarity of the cells ${ }^{14}$, which alters the expression of transporters. Consequently, passive and active transport cannot be strictly separated. In addition, active transport mechanisms may also become impaired, by which different effects may occur. Damage to influx transporters will cause a deficit in the brain, while damage to efflux transporters will lead to a buildup of a certain substance. As this is a disruption of an active process, this shall be referred to as BBB dysfunction in this review. BBB impairment may either be due to increased permeability or due to dysfunction of active transport mechanisms.

The metabolism of the brain also depends on the circulation of CSF, which is produced in the choroid plexus in the lateral ventricles and is absorbed by the arachnoid granulations into the venous sinuses. At the interface between blood and CSF in the choroid plexus resides the blood-CSF barrier, and at the brain and CSF interface exists the brain-CSF barrier, both of which also exhibit tight junctions to limit transport of molecules to and from the brain tissue ${ }^{14}$. Although impairment of these barriers is not within the scope of this review, it is important to state that neurotoxic compounds may also flow from the blood into the CSF and subsequently into the brain.

\section{BBB dysfunction in dementia}

Different hypotheses exist on the specific role of the BBB in $A D$. A popular candidate for finding the link between the $B B B$ and $A D$ is $A B^{15}$. An intact $B B B$ will properly regulate $A B$ using active transport mechanisms to control its in- and outflow ${ }^{16}$. The effect of $A B$ in $A D$ and its specific effect on the BBB has been described in detail in a recent review by Erickson and Banks ${ }^{10}$. They concluded that $A B$ accumulation only partially explains $B B B$ impairment in Alzheimer's dementia. It was hypothesized that $A B$ is neurotoxic and enters the brain through an impaired BBB, and subsequently induces neurodegeneration ${ }^{17,18}$. As described in another review by Burgmans et al. ${ }^{9}$, BBB dysfunction and accumulation of $A B$ in the brain tissue may reinforce each other through different processes: $A B$ accumulation causes $B B B$ dysfunction through endothelial toxicity and an 
increase in monocyte adhesion. On the other hand, $B B B$ dysfunction causes $A B$ accumulation through different mechanisms: through leakage of $A B$ through the $B B B$, through an insufficient clearance of $A B$ from the brain tissue, and through induced $A B$ precursor protein cleavage ${ }^{19}$. BBB dysfunction and $A B$ accumulation would then cause vascular pathology and $A B$ depositions, respectively, which leads to neurodegeneration and dementia ${ }^{4}$. Although the exact role of the BBB in the pathogenesis of dementia remains unclear, there is substantial evidence for BBB dysfunction in dementia.

\section{Evidence from non-imaging studies}

\section{Postmortem evidence}

The earliest evidence for microvascular pathology in dementia comes from the postmortem histological examination of brain tissue, which has also become the golden standard for diagnosis of the disease causing the dementia. Using this technique, Alois Alzheimer already described arteriosclerosis, neurofibrillary tangles, and plaques in the brain of one of his patients, who had severe memory impairment before death. With respect to the $\mathrm{BBB}$, more recent postmortem investigations of dementia showed mixed results, with some finding $B B B$ damage ${ }^{8,20-29}$, while others did not finding any evidence of a compromised $\mathrm{BBB}^{30-33}$. Additional evidence comes from studies which have investigated the claudin expression profile of $A D$ patients. Claudin is a protein that is essential for the formation and functioning of the tight junctions ${ }^{34}$. An overexpression of claudin in the brain of patients with $A D$ was found, which could indicate a response to cellular stress or vascular dysfunction, and likely has an effect on the $\mathrm{BBB}^{35,36}$.

Although postmortem measures can provide valuable insight into the underlying cause of $A D$, only in vivo imaging methods can show us the extent and severity of the problem during life, while dealing with the effects of the circulation and homeostasis. Postmortem methods can be able to accurately pinpoint the site and extent of BBB dysfunction. However, postmortem methods have two main disadvantages. First, BBB dysfunction may be a postmortem effect, resulting from the absence of circulation and homeostasis ${ }^{31}$. Second, acquiring brains suffering from $A D$ at an early stage is difficult, as patients usually live for years after initial onset of the symptoms and aging has a significant impact on BBB function ${ }^{37}$. Therefore, postmortem studies generally look at an end stage of the disease, while investigation of dementia at an early stage and during life may prove more informative. 


\section{Animal studies}

There are different animal models available which mimic the human AD phenotype quite closely ${ }^{38}$. Common examples are transgenic mouse models which develop a familial form of $A D$. These mice often also display cerebrovascular pathology ${ }^{39}$, indicating interplay between $A B$ pathology and the cerebral blood vessels. Such models, in combination with different techniques for evaluation of the BBB (see also Wunder et al. ${ }^{40}$ ), have been used to show different pathways through which the $B B B$ and $A B$ interact with each other'. The animal studies have supplied a sizeable portion of the evidence in support of the hypothesized importance of BBB dysfunction in dementia. However, even though animal studies supply the possibility of validation of BBB assessment techniques and invasive imaging, the translation of results to human pathology remains difficult, especially since the cause of cognitive decline in dementia is probably multifactorial ${ }^{2}$.

\section{Albumin ratio}

A commonly used in vivo measure for BBB impairment is the albumin ratio, which is defined as the concentration of albumin found in the cerebrospinal fluid (CSF) divided by the concentration of serum albumin. Albumin is a relatively large molecule (molecular weight, $67 \mathrm{kDa}$ ) that circulates in the blood and can only enter the CSF by crossing the BBB. The amount of albumin crossing the healthy BBB is strictly regularized. Therefore, an increased albumin ratio is considered to be a measure of BBB impairment ${ }^{41,42}$. CSF is obtained using a lumbar puncture, a common, but somewhat burdening, procedure.

An overview of different studies utilizing the albumin ratio to investigate the BBB is given in Table 2.1. The listed studies on AD provide convincing evidence of albumin leakage. In the literature we found 9 studies on $A D$ supporting leakage of albumin into the $\mathrm{CSF}^{43-50}$. The higher albumin ratio was also found in studies investigating patients with vascular dementia ${ }^{46,48,49,51-53}$. However, two studies could not find a significant difference between demented patients and healthy controls ${ }^{54,55}$. Together, the majority of findings suggest that a higher albumin ratio is often present in patients suffering from dementia, especially those exhibiting vascular pathology. However, a disadvantage of the albumin ratio is that it does not provide insight into the localization of leakage, as it is a global measure. Also, it does not necessarily reflect BBB impairment. For instance, the leakage of albumin could also come from blood-CSF barrier defects, while the BBB could remain intact. Additionally, the albumin ratio may be increased when there is 
albumin leakage through spinal cord vessels, subarachnoid vessels or the spinal cord, which cannot be differentiated from BBB leakage.

\section{Evidence from imaging studies}

So far, postmortem, animal model, and albumin ratio investigations have provided substantial information for $B B B$ dysfunction and on the role of the $B B B$ in the pathophysiology of $A D$. This evidence is mainly indirect however, without proof of the exact location of BBB defects, and illustrates the need for methods that examine the $\mathrm{BBB}$ in vivo and in a spatially resolved way. Both of these requirements can be met by using imaging techniques performed with a contrast agent, which is why the literature was searched for applications of such methods in patients with dementia.

\section{Search criteria}

In this study, we investigated the hypothesis of BBB dysfunction in dementia through a literature review. In particular, we focused on in vivo imaging methods to assess the BBB in dementia.

Two sources, PubMed and Web of Science, were used to search for literature on the subject of localized in vivo BBB integrity measurements in people with dementia.

We searched PubMed for articles which had the following terms in either the title, abstract or as a keyword: "blood-brain barrier", "Alzheimer", "mild cognitive impairment", "dementia“, "permeability", "integrity” and "function". From the Web of Science we included articles which had the following terms as a topic: "blood-brain barrier", "Alzheimer", "mild cognitive impairment", "dementia“, "permeability", "integrity" and "function", excluding articles which used animals or in vitro techniques. 
Table 2.1: Summary of albumin ratio studies assessing the BBB in dementia

\begin{tabular}{|c|c|c|c|}
\hline $\begin{array}{l}\text { Investigated } \\
\text { disorder, group size }\end{array}$ & $\begin{array}{l}\text { Mean age }(y) \text { of } \\
\text { patients, } \\
\text { controls }\end{array}$ & Main findings & Reference \\
\hline $32 \mathrm{AD}$ vs. $22 \mathrm{HC}$ & 69,67 & Higher albumin ratio & $\begin{array}{l}\text { Elovaara et al., } \\
1987\end{array}$ \\
\hline $\begin{array}{l}64 \mathrm{AD} \text { vs. } 17 \mathrm{VaD} \text { vs. } \\
52 \mathrm{HC}\end{array}$ & $65,68,43$ & $\begin{array}{l}\text { No higher albumin ratio in } A D \text {, } \\
\text { higher in } \mathrm{VaD}\end{array}$ & $\begin{array}{l}\text { Leonardi et al. } \\
1985\end{array}$ \\
\hline $\begin{array}{l}22 \mathrm{AD} \text { vs. } 29 \mathrm{VaD} \text { vs. } \\
22 \mathrm{HC}\end{array}$ & $67,68,67$ & $\begin{array}{l}\text { Higher albumin ratio in } A D \text { and } \\
\text { VaD compared to controls but } \\
\text { not between } A D \text { and VD }\end{array}$ & $\begin{array}{l}\text { Elovaara et al., } \\
1987\end{array}$ \\
\hline $31 \mathrm{AD}$ vs. $14 \mathrm{HC}$ & 66,65 & No difference in albumin ratio & $\begin{array}{l}\text { Kay et al., } \\
1987\end{array}$ \\
\hline $53 \mathrm{VaD}$ vs. $30 \mathrm{HC}$ & 74,72 & Higher albumin ratio in $\mathrm{VaD}$ & $\begin{array}{l}\text { Wallin et al., } \\
1990\end{array}$ \\
\hline $118 \mathrm{AD}$ vs. $50 \mathrm{HC}$ & 72,72 & $\begin{array}{l}\text { Higher albumin ratio in } A D \\
\text { when combined with vascular } \\
\text { pathology }\end{array}$ & $\begin{array}{l}\text { Blennow et al., } \\
1990\end{array}$ \\
\hline $\begin{array}{l}13 \text { EOAD vs. } 33 \text { AD vs. } \\
20 \text { VaD vs. } 17 \mathrm{HC}\end{array}$ & $64,77,72,60$ & Higher albumin ratio in $\mathrm{VaD}$ & $\begin{array}{l}\text { Mecocci et al., } \\
1991\end{array}$ \\
\hline $25 \mathrm{AD}$ vs. $25 \mathrm{HC}$ & 69,69 & $\begin{array}{l}\text { Higher absolute albumin in } \\
\text { serum in } A D\end{array}$ & $\begin{array}{l}\text { Frölich et al., } \\
1991\end{array}$ \\
\hline $\begin{array}{l}45 \mathrm{AD} \text { vs. } 30 \mathrm{VaD} \text { vs } \\
23 \text { OND vs. } 27 \mathrm{HC}\end{array}$ & $69,70,63,63$ & $\begin{array}{l}\text { No higher albumin ratio } \\
\text { between the groups }\end{array}$ & $\begin{array}{l}\text { Mattila et al., } \\
1994\end{array}$ \\
\hline $\begin{array}{l}13 \text { AD vs. } 14 \text { VaD vs. } 2 \\
\text { other dementia vs. } 29 \\
\text { HC }\end{array}$ & $85,85,85,85$ & $\begin{array}{l}\text { Higher albumin ratio in } A D \text { and } \\
\operatorname{VaD}\end{array}$ & $\begin{array}{l}\text { Skoog et al., } \\
1998\end{array}$ \\
\hline $\begin{array}{l}14 \mathrm{AD} \text { vs. } 7 \mathrm{VaD} \text { vs } 7 \\
\mathrm{HC}\end{array}$ & $78,74,79$ & $\begin{array}{l}\text { Higher albumin ratio in } A D \text { and } \\
\operatorname{VaD}\end{array}$ & Wada, 1998 \\
\hline $42 \mathrm{AD}$ & 70 & $\begin{array}{l}\text { Higher albumin correlates with } \\
\text { hippocampal atrophy }\end{array}$ & $\begin{array}{l}\text { Matsumoto et } \\
\text { al., } 2007\end{array}$ \\
\hline $36 \mathrm{AD}$ & 71 & High albumin in $22 \%$ & $\begin{array}{l}\text { Bowman et al., } \\
\text { 2007b }\end{array}$ \\
\hline $157 \mathrm{AD}$ & 70 & $\begin{array}{l}\text { High albumin ratio in } 42 \% \text { of } \\
\text { men and } 13 \% \text { of women }\end{array}$ & $\begin{array}{l}\text { Algotsson and } \\
\text { Winblad, } 2007\end{array}$ \\
\hline $36 \mathrm{AD}$ & 70 & High albumin ratio in $22 \%$ & $\begin{array}{l}\text { Bowman et al., } \\
2012\end{array}$ \\
\hline
\end{tabular}

AD: Alzheimer's Disease; HC: Healthy Controls; VaD: Vascular Dementia; FLD: Frontotemporal Lobe Dementia; PDD: Primary Degenerative Dementia; MBs: Microbleeds; PgP: P-glycoprotein; EOAD: Early Onset Alzheimer's Disease; OND: Other Neurological Disorders 
We also excluded non-English articles, reviews, animal studies and articles from before 1985. Based on title and abstract, we excluded studies not focusing on (a form of or presumed pre-stage of) dementia and we only included in vivo human studies where BBB dysfunction was measured in the human brain in a way that the dysfunction was spatially definable. We cross-referenced other reviews to make sure we did not miss any studies $^{5,15,37}$.

The PubMed search resulted in 166 hits; the Web of Science search resulted in 302 hits. There was an overlap between the search results of 34 articles. After reading the title and abstract of each article, 11 studies remained.

\section{Search results}

From our literature search, we identified the following studies using in vivo imaging techniques to investigate the BBB: Bronge and Wahlund, 2000; Caserta et al., 1998; Wang et al., 2006; Dysken et al., 1990; Hanyu et al., 2002; Schlageter et al., 1987; Starr et al., 2009; Taheri et al., 2011a, 2011b; van Assema et al., 2012a, 2012b ${ }^{56-66}$. Of these studies, 6 used gadolinium-based contrast agents for MRI ${ }^{56,59,61-64}, 2$ used iodine-based contrast agents for $\mathrm{CT}^{57,58}$, and 3 used radioactive tracers for $\mathrm{PET}^{60,65,66}$. An overview of these studies can be found in Table 2.2, and the results are discussed in the following sections.

In all these studies, the BBB impairment was measured inside human brains by means of neuroimaging techniques using a contrast reagent; either a passively leaking contrast agent for CT or MRI, or an actively transporting radiotracer for PET. For CT or MRI, the choice of applicable contrast agent differs per imaging modality. Moreover, the suitability differs per type of contrast agent as the BBB permeability depends, among other properties, on the size and lipophilicity of the compound ${ }^{12,13}$. Ideally, a contrast agent should not or barely pass an intact BBB, but should easily cross a damaged (i.e. leaky) BBB. As there are large differences between the analyses used in the studies, a brief overview of common contrast-enhanced imaging methodology is given in the following paragraphs.

Dynamic contrast-enhanced imaging relies on the assessment of a temporal gradient in contrast agent concentration, by which the transfer rate of the contrast agent from the intravascular space into the extravascular extracellular space can be obtained as a measure of BBB permeability. There are different ways to perform this assessment. The most straightforward method is to investigate the relative signal enhancement in a 
region of interest (ROI), which was used in 4 of the $6 \mathrm{MRI}$ studies. A stronger signal enhancement of the ROI inside the brain indicates a higher concentration of contrast agent, which is interpreted as leakage.

Another method is to use a pharmacokinetic model to analyze the contrast agent dynamics that cause specific enhancement of the brain tissue. This method was used in 1 of the 2 CT studies, 2 of the 6 MRI studies and all 3 of the PET studies. With modeling, the BBB leakage may be determined in a more quantitative manner. The numerously available models differ in complexity and the number of assumptions under which they can be applied ${ }^{67}$. The most important difference between models is the amount of compartments that are considered. Commonly, a two-compartment model is used (see Figure 2.4), which assumes that each ROI contains a vascular compartment and an extravascular extracellular space compartment. Also important to note is that some models (such as the Patlak model ${ }^{68}$ ) do not take reflux of the contrast agent into account, which could lead to misinterpretation of the data. Significant reflux is very likely in high leakage scenarios such as in high-grade tumors (for an example see Figure 2.5). The application of these methods in the included studies is discussed in the following paragraphs. 
Table 2.2: Summary of in vivo imaging studies assessing the BBB in dementia

\begin{tabular}{|c|c|c|c|c|}
\hline $\begin{array}{l}\text { Investigated } \\
\text { disorder, } \\
\text { group size }\end{array}$ & $\begin{array}{l}\text { Mean age } \\
\text { (y) of } \\
\text { patients, } \\
\text { controls }\end{array}$ & Type of assessment & Main findings & Reference \\
\hline $\begin{array}{l}\mathrm{AD} / \mathrm{MCl} / \mathrm{SCl} / \\
\mathrm{VaD} / \mathrm{FLD}, 5 \\
\text { with } \\
\text { elevated AR } \\
\text { vs. } 5 \text { with } \\
\text { normal AR }\end{array}$ & 73,72 & $\begin{array}{l}\text { CE-MRI, signal } \\
\text { enhancement } \\
\text { ratio, max } 25 \text { mins }\end{array}$ & $\begin{array}{l}\text { No increased signal } \\
\text { enhancement in WMLs } \\
\text { compared to normal } \\
\text { appearing white matter } \\
\text { both in elevated AR } \\
\text { and normal AR }\end{array}$ & $\begin{array}{l}\text { Bronge and } \\
\text { Wahlund, } 2000\end{array}$ \\
\hline $\begin{array}{l}17 \text { BD vs. } 10 \\
\text { ICE vs. } 14 \\
\text { HC }\end{array}$ & $78,77,79$ & $\begin{array}{l}\text { CE-MRI, T1 } \\
\text { relaxation time } \\
\text { measurements, } \\
\text { max } 15 \text { mins }\end{array}$ & $\begin{array}{l}\text { BBB leakage only in } \\
\text { WMLs in patients with } \\
\text { BD }\end{array}$ & $\begin{array}{l}\text { Hanyu et al., } \\
2002\end{array}$ \\
\hline $\begin{array}{l}11 \mathrm{MCl} \text { vs. } \\
10 \mathrm{HC}\end{array}$ & 74,74 & $\begin{array}{l}\text { CE-MRI, signal } \\
\text { enhancement } \\
\text { ratio, max } 5 \text { mins }\end{array}$ & $\begin{array}{l}\text { BBB leakage in } \\
\text { hippocampus }\end{array}$ & $\begin{array}{l}\text { Wang et al., } \\
2006\end{array}$ \\
\hline $\begin{array}{l}15 \text { AD vs. } 15 \\
H C\end{array}$ & 74,73 & $\begin{array}{l}\text { CE-MRI, signal } \\
\text { enhancement, } \\
\text { NEX=9, max } 30 \\
\text { mins }\end{array}$ & $\begin{array}{l}\text { signal enhancement } \\
\text { indicative of different } \\
\text { blood-brain-CSF } \\
\text { compartment kinetics }\end{array}$ & $\begin{array}{l}\text { Starr et al., } \\
2009\end{array}$ \\
\hline $\begin{array}{l}45 \mathrm{VCl} \text { vs. } \\
20 \mathrm{HC}\end{array}$ & 64,51 & $\begin{array}{l}\text { CE-MRI, modified } \\
\text { Patlak model, max } \\
23 \text { mins }\end{array}$ & $\begin{array}{l}\text { Selective BBB leakage } \\
\text { in } \mathrm{VCl}\end{array}$ & $\begin{array}{l}\text { Taheri et al., } \\
\text { 2011b }\end{array}$ \\
\hline $\begin{array}{l}60 \mathrm{VCl} \text { vs. } \\
17 \mathrm{HC}\end{array}$ & 63,44 & $\begin{array}{l}\text { CE-MRI, modified } \\
\text { Patlak model, max } \\
23 \text { mins }\end{array}$ & $\begin{array}{l}\text { BBB leakage compared } \\
\text { to controls }\end{array}$ & $\begin{array}{l}\text { Taheri et al., } \\
\text { 2011a }\end{array}$ \\
\hline $\begin{array}{l}26 \text { PDD vs. } \\
15 \mathrm{HC}\end{array}$ & 67,62 & $\begin{array}{l}\text { CE-CT, washout } \\
\text { curves, max } 35 \mathrm{~s}\end{array}$ & $\begin{array}{l}\text { No BBB leakage } \\
\text { observed }\end{array}$ & $\begin{array}{l}\text { Dysken et al., } \\
1990\end{array}$ \\
\hline $\begin{array}{l}14 \text { AD vs. } 9 \\
H C\end{array}$ & 87,83 & $\begin{array}{l}\text { CE-CT, two } \\
\text { compartment } \\
\text { model, max } 60 \\
\text { mins }\end{array}$ & $\begin{array}{l}\text { No BBB leakage } \\
\text { observed }\end{array}$ & $\begin{array}{l}\text { Caserta et al., } \\
1998\end{array}$ \\
\hline $\begin{array}{l}5 \mathrm{AD} \text { vs. } 5 \\
\mathrm{HC}\end{array}$ & 66,68 & $\begin{array}{l}\text { PET, [68Ga]EDTA } \\
\text { tracer, Patlak } \\
\text { model }\end{array}$ & $\begin{array}{l}\text { No BBB leakage } \\
\text { observed }\end{array}$ & $\begin{array}{l}\text { Schlageter et } \\
\text { al., } 1987\end{array}$ \\
\hline $\begin{array}{l}13 \text { AD vs. } 14 \\
H C\end{array}$ & 65,62 & $\begin{array}{l}\mathrm{PET},(\mathrm{R})- \\
{\left[{ }^{11} \mathrm{C}\right] \text { verapamil }} \\
\text { tracer for PgP } \\
\text { function, max } 60 \\
\text { mins }\end{array}$ & $\begin{array}{l}\text { PgP function decreased } \\
\text { in } A D\end{array}$ & $\begin{array}{l}\text { van Assema et } \\
\text { al., 2012b }\end{array}$ \\
\hline $\begin{array}{l}12 \mathrm{AD} \text { vs. } 6 \\
A D+M B s\end{array}$ & 66,63 & $\begin{array}{l}\text { PET, }(\mathrm{R})- \\
{\left[{ }^{11} \mathrm{C}\right] \text { verapamil }} \\
\text { tracer for PgP } \\
\text { function, max } 60 \\
\text { mins }\end{array}$ & $\begin{array}{l}\text { No increased PgP } \\
\text { dysfunction observed in } \\
A D+M B s \text { vs. } A D\end{array}$ & $\begin{array}{l}\text { van Assema et } \\
\text { al., 2012a }\end{array}$ \\
\hline
\end{tabular}

AD: Alzheimer's Disease; BD: Binswanger's Disease; ICE: Ischemic Cerebrovascular Events; CE: Contrast Enhanced; WMLs: White Matter Lesions; MCI: Mild Cognitive Impairment; SCI: Subjective Cognitive Impairment; VaD: Vascular Dementia; FLD: Frontotemporal Lobe Dementia; AR: Albumin Ratio; HC: Healthy controls; PDD: Primary Degenerative Dementia; MBs: Microbleeds; PgP: Palvcoprotein: NEX: Number of EXcitations 


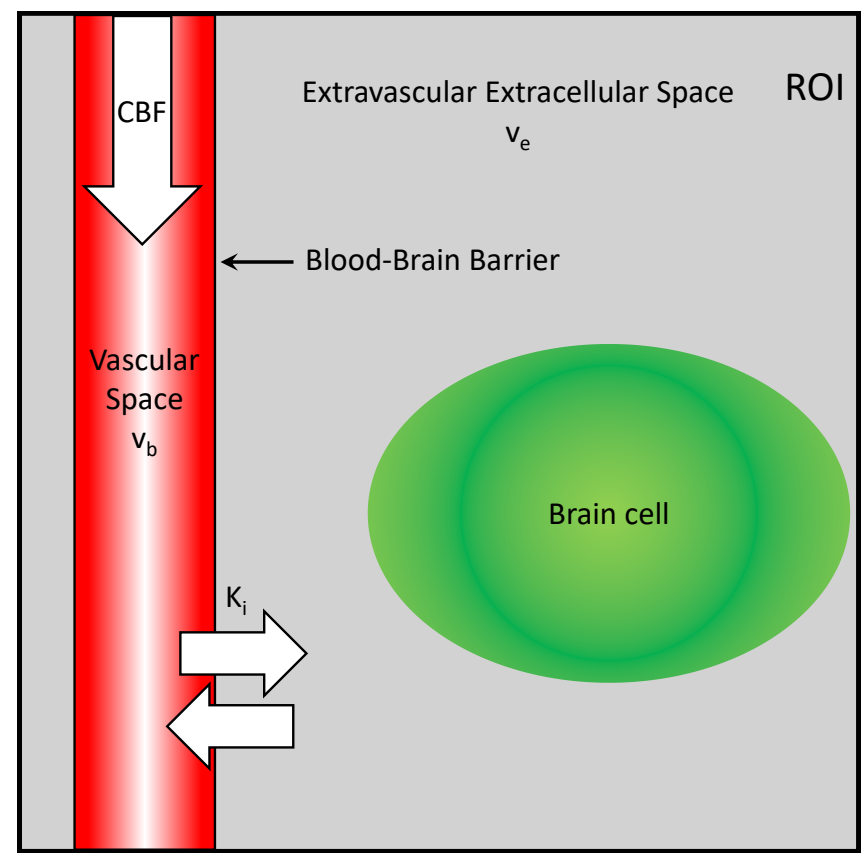

Figure 2.4: Schematic overview of a two-compartment model in brain tissue. The region of interest (ROI) can either be a voxel or a multitude of voxels. Each ROI contains a blood compartment with volume $v_{b}$, and an extravascular extracellular compartment with volume $v_{e}$, into which the contrast agent leaks. These two compartments are separated by the blood-brain barrier. The cerebral blood flow (CBF) is the amount of blood per time unit entering and exiting the ROI. The transfer rate of the contrast agent into the extravascular extracellular compartment is denoted as $\mathrm{K}_{\mathrm{i}}$.

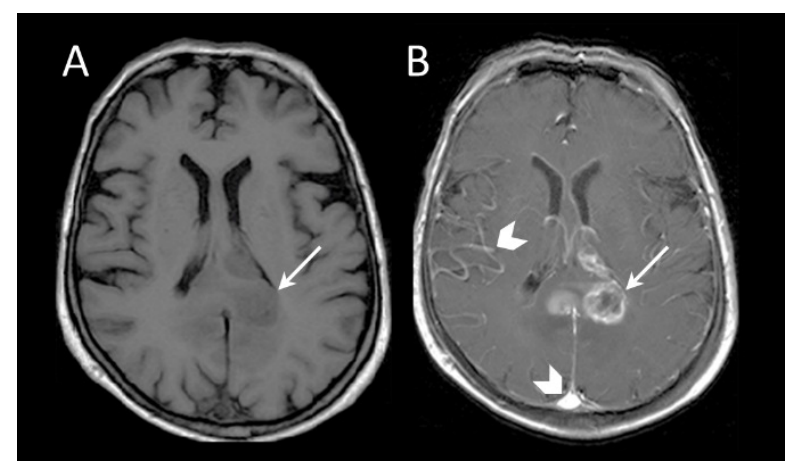

Figure 2.5: T1 weighted images of a patient with a tumor (arrow) before (A) and after contrast injection (B), illustrating the signal enhancement both in the tumor and in the blood vessels such as the sagittal sinus and some arteries (arrowheads). 
Magnetic resonance imaging

Magnetic Resonance Imaging is an imaging technique that relies on the measurement of the electromagnetic energy absorption and relaxation behavior of protons (water molecules) in a magnetic field. By using paramagnetic contrast agents, such as gadolinium based compounds, the relaxation behavior of the protons is altered in such a way that the MRI signal changes. One of the most common contrast agents in MRI is gadolinium-diethylenetriaminepentacetate (Gd-DTPA). It is a relatively small molecule (molecular weight, $0.6 \mathrm{kDa}$ ) which is thought to pass the BBB through the tight junctions if they are damaged ${ }^{69}$. Although gadolinium-based agents are considered relatively safe compared to iodine-based agents, there are rare reports of nephrogenic systemic fibrosis as a consequence of $\mathrm{Gd}$ administration ${ }^{70}$. The most widely used technique to investigate the BBB in vivo is dynamic contrast enhanced-MRI (DCE-MRI), which applies dynamic imaging to visualize contrast agent distribution over time.

Wang et al. ${ }^{64}$ used DCE-MRI in patients with $\mathrm{MCl}$ to investigate the hippocampus, with the cerebellum as a reference. Signal enhancement in the cerebellum and left hippocampus appeared not to be different between patients with $\mathrm{MCl}$ and healthy controls. However, in the right hippocampus the signal enhancement curve was less steep and was higher after contrast agent administration in the patients compared to controls. They interpreted this as a lower local blood volume and longer signal retention, indicative of vascular changes in the hippocampus rather than an indication of BBB permeability. Starr et al. ${ }^{61}$ used DCE-MRI in 15 patients diagnosed with probable AD and their healthy spouses. They placed multiple ROls throughout the brain and showed aberrant temporal patterns of gadolinium-based signal enhancement, indicating altered blood-brain-CSF compartment kinetics. They also discussed whether the likely pathway of the contrast agent accumulation in the brain was blood-CSF-brain, or blood-brainCSF. Based on their results, they concluded that the latter pathway was more plausible in the timeframe (30 minutes) of the study. Unfortunately, no other study reported similar results, so further confirmation of this finding is needed. 


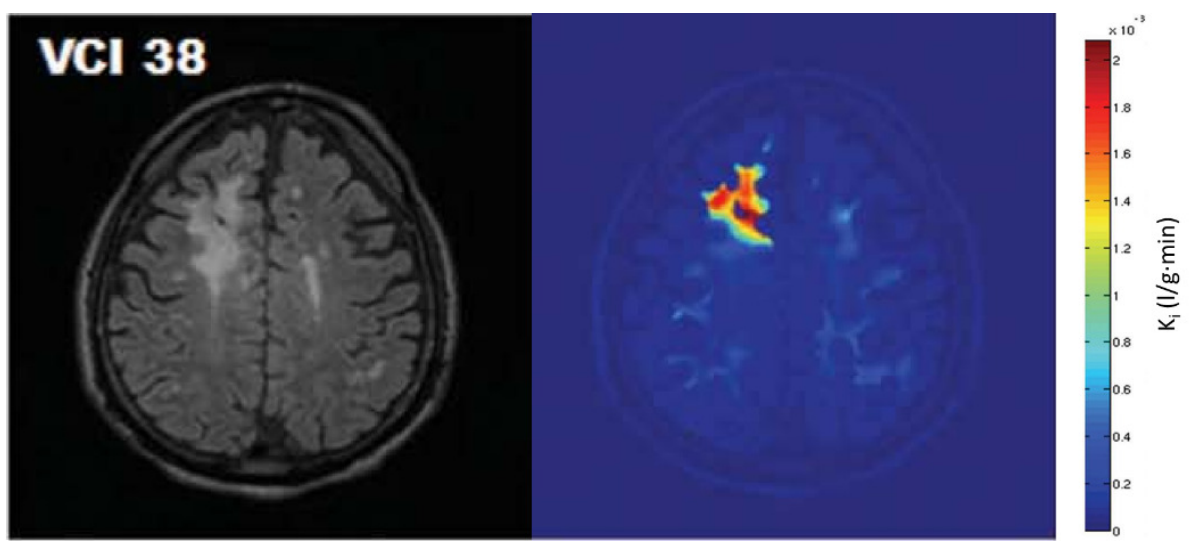

Figure 2.6: An example of BBB leakage as shown by Taheri et al. in a $\mathrm{VCl}$ patient. On the left is a FLAIR image of a patient with vascular cognitive impairment. The image on the right displays a colorcoded BBB leakage map ${ }^{62}$.

Using DCE-MRI, Bronge and Wahlund ${ }^{56}$ focused on white matter hyperintensities (WMHs) in dementia to investigate the role of WMHs in the pathophysiology of dementia. They examined 10 patients with WMHs, 5 with elevated and 5 with normal albumin ratio. However, no significant difference was found in the WMHs between the two groups. Hanyu et al. ${ }^{59}$ also used DCE-MRI in patients with Binswanger's disease (a rare, more diffuse and subcortical form of vascular dementia ${ }^{71}$ ). They analyzed periventricular WMHs in these patients and compared the results to periventricular WMHs in patients with ischemic cerebrovascular events and normal appearing white matter of healthy control subjects by measuring T1 relaxation times before and after contrast injection. The $\mathrm{T} 1$ relaxation time, which shortens with increased contrast agent concentration, was shorter in the patients with Binswanger's disease compared to those in the WMHs of the patients with ischemic cerebrovascular events and to the normal appearing white matter in the control group. The lowered relaxation times in the patients with Binswanger's disease also correlated with the mini-mental state evaluation test score, indicating a link between BBB dysfunction in WMHs in Binswanger's disease and cognitive function.

Two studies by Taheri et al.62,63 investigated BBB dysfunction in relatively large groups ( $n=45$ and 60 , respectively) of patients with vascular cognitive impairment ( $\mathrm{VCl}$ ) using DCE-MRI. In both studies they used a postcontrast scanning time of 24.5 minutes and the 
Patlak graphical approach was used to calculate BBB permeability and local blood volume maps. The resulting leakage maps were investigated by means of distribution histograms. They demonstrated an increased contrast agent leakage localized in the center of some WMHs, though not in every WMH, nor in its periphery (for an example, see Figure 2.6). An explanation for this not specific localization of BBB leakage was not given. Overall, the MRI based studies provide evidence for BBB dysfunction in $A D$, especially in patients with WMHs and vascular pathology.

\section{Computed tomography}

Contrast agents in computed tomography (CT) are often iodine-based and have a much stronger absorption of X-rays compared to, for instance, bone or soft tissue. This way, the distribution of the contrast agent becomes visible on a CT image.

In our literature search we found 2 studies that used contrast-enhanced CT to investigate possible BBB dysfunction in dementia ${ }^{57,58}$. Dysken et al. ${ }^{57}$ used CT to investigate primary degenerative dementia. For this study, multiple ROls were placed to determine the signal intensity course over time. However, no significant differences were found between the primary degenerative dementia patients and healthy controls. The dynamic CT measurement lasted up to $35 \mathrm{~s}$ after contrast administration, which is rather short compared to other studies using contrast-enhanced imaging (Table 2.2). If any weak BBB leakage exists, it would be more easily detected when the total measurement would be longer. A second CT study is by Caserta et al. ${ }^{58}$, who used a two compartment model for evaluating the temporal signal changes in a number of ROl's in the gray and white matter. However, none of the pharmacokinetic measures appeared different between the patients with (probable) $A D$ and healthy controls. In sum, the number of contrastenhanced CT studies is limited, and they do not provide any evidence for BBB impairment in dementia.

\section{Positron emission tomography}

Positron Emission Tomography (PET) is a technique which utilizes an administered radioactive tracer (radiopharmacon) that emits energetic gamma photons (511 keV) that can be detected in a spatially resolved way. Radiopharmacons can be designed to bind to specific biomolecules expressed in certain diseases. The most common PET tracer $18 \mathrm{~F}$-Fluorodeoxyglucose (FDG) can be used to investigate glucose metabolism in the brain. The uptake of FDG in the brain is usually very strong and lowered in certain brain regions for patients with dementia ${ }^{72}$. However, because FDG-PET cannot differentiate 
between a dysfunctional BBB through a lowered expression of the GLUT-1 transporter (an influx transporter which regulates the active transport of glucose across the $\mathrm{BBB}$ ) and a decreased glucose metabolism rate, it seems an unsuitable technique for BBB assessment. Also, evidence indicates that the lower expression of the GLUT-1 transporter is a consequence of $A D$, rather than a caus $\mathrm{e}^{10}$.

There are multiple pathways through which $A B$ is cleared from the brain, but the main receptors are low-density lipoprotein 1 , the receptor for advanced glycation end products (RAGE), and P-glycoprotein ${ }^{73,74}$. Van Assema et al. used (R)- $\left[{ }^{11} \mathrm{C}\right]$ verapamil, which binds to $\mathrm{P}$-glycoprotein binding sites, in 15 patients with $A D^{65,66}$. The binding potential of $(R)-\left[{ }^{11} C\right]$ verapamil in gray matter was stronger in patients than healthy controls which indicated that $A B$ clearance was lowered in $A D$. They also investigated $A D$ patients without evidence of microbleeds compared to those who had microbleeds. The patients with microbleeds did not show any additional $A B$ clearance problems, which indicates that lower clearance of $A B$ is not linked to microbleeds in $A D$.

A possible tracer for (passive) BBB permeability using PET is [68Ga]ethylenediaminetetraacetic acid ([68Ga]EDTA $)^{75}$ (molecular weight $0.3 \mathrm{kDa}$ ), which is biologically inert and should not pass the intact BBB (Hawkins et al., 1984). An advantage of using tracers such as [68Ga]EDTA is that much lower concentrations of contrast reagent can be used compared to other common contrast agents used for $\mathrm{CT}$ or $\mathrm{MRI}^{76}$. This tracer has been used by Schlageter et al. to investigate BBB permeability in five patients with $A D$ compared to five healthy controls ${ }^{60}$. They used a two-compartment kinetic model to analyze the decay curves of [68Ga]EDTA in ROls located in the left and right hemispheres. No difference was found between patients and controls and they suggest that a more efficient scanner and higher dose might improve sensitivity to small leakage. Taken together, PET imaging has provided unique insight into BBB function in AD with regard to the disruption of $A B$ clearance pathways.

\section{Promising future methods}

Currently, detailed knowledge on the nature of BBB impairment in dementia is lacking due to the fact that most of the imaging studies have focused on tight junction damage, while many other $B B B$ pathways related to active transport of molecules may be impaired. The most convincing evidence for BBB impairment in dementia comes from MRI studies which used pharmacokinetic modeling in patients with vascular pathology. Expanding on this, we will discuss methodology improvements for existing techniques 
and propose underutilized methods which can be used to image defects of the BBB in vivo.

\section{DCE-MRI}

DCE-MRI methodology can be improved to increase the sensitivity to BBB permeability. Most progress can be made by increasing signal-to-noise ratio (SNR) or by increasing spatial resolution. A higher SNR would help to improve the sensitivity to low concentrations of gadolinium due to limited BBB leakage, and a better spatial resolution would help to detect small "hot spots" of leakage. Moving to higher field strengths such as $7 \mathrm{~T}$ may help in this regard ${ }^{77,78}$. Also, a multicompartment model ${ }^{68,79}$, combined with proper contrast agent concentration calculations ${ }^{80}$ and whole-brain voxel-wise analysis methods, should increase sensitivity for low and localized leakage.

\section{Microvascular water exchange MRI methods}

Water balance is an important part of the brain homeostasis. Water transport across the BBB is mostly osmotically driven through active uptake of essential salts, but distribution of water is regulated by aquaporins, which reside mainly on the astrocytic endfeet ${ }^{14,81,82}$. If the aquaporin function is impaired, the water balance may be disturbed and transport of water molecules across the BBB may be altered. Besides DCE-MRI, the water regulation can also be evaluated without the use of a contrast agent by using the MRI technique intravoxel incoherent motion $(I V I M)^{83}$. Using IVIM, it is possible to get an indication of BBB aquaporin (dys)function ${ }^{84}$.

\section{PET}

One of the main advantages of PET imaging is that it allows for a very detailed evaluation of metabolic cell processes by binding radioactive labels to specific molecules ${ }^{40}$. This would allow for imaging of specific processes related to BBB function, possibly pinpointing which processes are interrupted in dementia. An example of how tracers may directly interact with specific components of the BBB, are amyloid transporter tracers ${ }^{65,66}$, such as $11 \mathrm{C}$-, 131 - or $18 \mathrm{~F}$-labeled $A B^{85-87}$. These tracers can be used to study intravascular and extravascular $A B$ concentrations. In future studies, specific components and transporters of the BBB can be investigated using similar methods for other transport mechanisms (e.g. the monocarboxylate transporter 1 for lactate and the amino acid transporters). 


\section{Discussion}

\section{Current state}

Most of the imaging of BBB impairment has focused on MRI rather than CT or PET, which are rarely used so far. PET studies show an impaired P-glycoprotein function but not a leaky or damaged BBB per se, while CT studies so far fail to show any effect. MRI studies do show evidence of BBB leakage in patients with dementia. This has particularly been found in patients with WMHs and vascular pathology. Actual BBB impairment in nonvascular forms of dementia, including pure $A D$, has not been reported so far using in vivo imaging. Nevertheless, since most patients with dementia have mixed pathologies, including a certain degree of vascular pathology ${ }^{88}$, it is difficult to distinguish vascular and non-vascular dementia. We therefore cannot conclude that BBB impairment does not exist in pure $A D$.

Our knowledge of BBB dysfunction in dementia from imaging studies is still in an early phase. This is illustrated by several problems. First, there are only 11 studies of in vivo BBB imaging in humans and more studies are prompted for. Second, the investigated patient groups are highly heterogeneous, as can be seen in Table 2.2. A total of seven different types of dementia were included in the studies using in vivo imaging. It would be advantageous to be able to meaningfully group the patients with dementia due to different causes. However, since that would require knowledge of the pathophysiology of dementia, this is currently not possible. Also, roughly half of the investigated patients in Table 2.2 suffered from vascular cognitive impairment ( $\mathrm{VCl}$ ), which implies that these patients already have cerebrovascular abnormalities. This $\mathrm{VCl}$ group is thus overrepresented. An additional problem is that postmortem histopathology often disagrees with clinical diagnosis ${ }^{88}$. It is known that pure $A D$ is rare, as patients often have mixed pathology, including vascular abnormalities. This hampers investigating the link between $A D$ and $B B B$ impairment, because it is almost impossible to exclude the modifying influence of vascular factors. Third, the subject groups investigated so far are relatively small. Larger patient groups should increase sensitivity to subtle effects. Fourth, the limited evidence for BBB leakage in dementia hints at a very slow and subtle kind of leakage, but this was not always considered in the studies investigating BBB impairment in dementia. Using a proper pharmacokinetic model instead of signal enhancement methods may improve sensitivity for such limited leakage. Moreover, it remains uncertain which compartments, blood, extravascular extracellular, and/or 
cerebrospinal fluid, need to be included in the modeling to obtain the most adequate quantification of the observable leakage. The signal enhancement ratio is useful to compare specific ROls, but the use of a pharmacokinetic model would allow for better comparison of various studies, and enables the separation of contrast enhancement by the filling of microvessel lumen from the leakage into the parenchyma.

In conclusion, the currently available literature does not provide sufficient insight into the nature and exact location of the BBB impairment in dementia. However, the data is indicative of (localized) BBB dysfunction in patients who already suffer from cerebrovascular pathology such as white matter lesions. Future research will have to prove the severity and extent of BBB dysfunction in dementia.

\section{Future directions}

Given its complex nature, future research should focus on the investigation of specific components of the BBB. Current research has predominantly focused on tight junction permeability and to some degree on the P-glycoprotein transporter. However, the BBB houses many more molecular transport mechanisms which, through a currently unknown cascade of events, may induce neurodegeneration and dementia. Also, since the loss of tight junctions influences the expression of active transporters, changes of passive transport mechanisms likely have more complicated effects on the entire BBB, making examination of separate transport systems even more challenging. As such, research focused on separate transport systems is needed to explore the exact pathway of the BBB pathology in dementia.

Transport specific research in AD needs novel imaging methods. Assessment of aquaporin function through water exchange measurements and the permeability of the tight junctions using DCE-MRI methods are relatively easy to perform. However, if these BBB components are not or only a small part of the $A D$ cascade, other techniques will have to be utilized. The most promising method is by using PET imaging with specific radiopharmacons that target specific transport receptors. Also, combining PET with MRI will open up even more routes of investigation. Using a PET-MRI, different contrast agents can be investigated at the same time, such as [68Ga]EDTA and Gd-DTPA. This allows for cross-validation of the contrast agents. It would also be possible to simultaneously measure cerebral blood flow with MRI and specific transporter function with PET to investigate interactions between ischemia and the BBB function. 
Transport pathway specific imaging is particularly relevant in an early phase of dementia. Early diagnosis would be very helpful for patient care and research. Currently, diagnosis of $A D$ during life is made mainly though neuropsychological evaluations of cognition. However, AD in its prodromal form may develop much sooner than the initial onset of cognitive decline ${ }^{89}$. The point at which patients consult a caregiver when they experience complaints may be long after the initial pathology starts. Imaging methods have the potential to make such early pathology visible. A complication is that patients suffering from prodromal $A D$ may never actually progress to full $A D^{90}$, which makes finding subjects in the very early stages of the disease even harder. Currently, the most reliable method to differentiate between the non-progressing and progressing groups is by observing the patient at a later stage of the disease when progress is already happening or has happened. Some recent $A B$ based PET tracers (11C-PiB ${ }^{86}$, 18Fflorbetapir ${ }^{91}$ and $18 \mathrm{~F}$-flutemetamo ${ }^{92}$ ) have shown promising results on early diagnosis on $\mathrm{AD}$. Although $11 \mathrm{C}-\mathrm{PiB}$ has been the most extensively studied amyloid tracer, its short half-life of 20 minutes is an evident practical limitation, as it has to be produced inhouse, which requires proper equipment and facilities. The development of fluorine tracers, which have a half-life of 110 minutes, has largely overcome this limitation. The most promising fluorine based amyloid tracers, florbetapir and flutemetamol, are still under development. If these future tracers will actually be able to provide an early diagnosis of $A D$, transport specific investigation at an early stage of the disease will become possible.

In conclusion, imaging studies on BBB function in dementia demonstrate BBB impairment, in particular in patients with vascular abnormalities. However, impairment in non-vascular forms of dementia, including $A D$, remains difficult to infer on. Future research should focus on larger patient groups and explicitly investigate the role of vascular abnormalities. There is also a lot of potential in examination of different BBB transport pathways. The BBB is a complex structure that plays an important role in brain homeostasis, so any specific transport pathway that is impaired may cause pathology. Transport pathway specific imaging using PET and investigation of $A D$ at an early stage will allow for a more detailed examination of the parts of the pathological cascade leading to dementia. 


\section{Acknowledgements}

We kindly thank May Wong, MSC and Eleana Zhang, MD for providing the images of Figure $2.1 \mathrm{~B}$ and $\mathrm{C}$. 


\section{References}

1 Pimplikar SW. Reassessing the amyloid cascade hypothesis of Alzheimer's disease. Int J Biochem Cell Biol 2009; 41: 1261-1268.

De La Torre JC. Is Alzheimer's disease a neurodegenerative or a vascular disorder? Data, dogma, and dialectics. Lancet Neurol 2004; 3: 184-190.

Hardy J, Selkoe DJ. The amyloid hypothesis of Alzheimer's disease: progress and problems on the road to therapeutics. Science 2002; 297: 353-356.

Hardy J. The amyloid hypothesis for Alzheimer's disease: A critical reappraisal. J Neurochem 2009; 110: 1129-1134.

Bell RD, Zlokovic BV. Neurovascular mechanisms and blood-brain barrier disorder in Alzheimer's disease. Acta Neuropathol 2009; 118: 103-113.

Deschaintre Y, Richard F, Leys D, Pasquier F. Treatment of vascular risk factors is associated with slower decline in Alzheimer disease. Neurology 2009; 73: 674-680.

Kalaria RN. The blood-brain barrier and cerebrovascular pathology in Alzheimer's disease. Ann $N$ Y Acad Sci 1999; 893: 113-125.

Wisniewski HM, Kozlowski PB. Evidence for blood-brain barrier changes in senile dementia of the Alzheimer type (SDAT). Ann N Y Acad Sci 1982; 396: 119-129.

Burgmans S, van de Haar HJ, Verhey FRJ, Backes WH. Amyloid-beta interacts with blood-brain barrier function in dementia: A systematic review. J. Alzheimer's Dis. 2013; 35: 859-873.

Erickson MA, Banks WA. Blood-brain barrier dysfunction as a cause and consequence of Alzheimer's disease. J Cereb Blood Flow Metab 2013; 33: 1500-13.

Zlokovic BV. The Blood-Brain Barrier in Health and Chronic Neurodegenerative Disorders. Neuron 2008; 57: 178-201.

Abraham MH. The factors that influence permeation across the blood-brain barrier. Eur J Med Chem 2004; 39: 235-240.

Seelig A, Gottschlich R, Devant RM. A method to determine the ability of drugs to diffuse through the blood-brain barrier. Proc Natl Acad Sci U S A 1994; 91: 68-72.

Abbott NJ, Rönnbäck L, Hansson E. Astrocyte-endothelial interactions at the blood-brain barrier. Nat Rev Neurosci 2006; 7: 41-53.

Wardlaw JM. Blood-brain barrier and cerebral small vessel disease. J Neurol Sci 2010; 299: 66-71.

Pahnke J, Walker LC, Scheffler K, Krohn M. Alzheimer's disease and blood-brain barrier function-Why have anti- $\beta$-amyloid therapies failed to prevent dementia progression? Neurosci Biobehav Rev 2009; 33: 1099-1108.

Deane R, Wu Z, Zlokovic BV. RAGE (yin) versus LRP (yang) balance regulates alzheimer amyloid betapeptide clearance through transport across the blood-brain barrier. Stroke 2004; 35: 2628-31.

Giulian D, Haverkamp L, Li J, Karshin WL, Yu J, Tom D et al. Senile plaques stimulate microglia to release a neurotoxin found in Alzheimer brain. Neurochem Int 1995; 27: 119-137.

Simons ER, Marshall DC, Long HJ, Otto K, Billingslea a, Tibbles $\mathrm{H}$ et al. Blood brain barrier endothelial cells express candidate amyloid precursor protein-cleaving secretases. Amyloid 1998; 5: 153-162. 
Claudio L. Ultrastructural features of the blood-brain barrier in biopsy tissue from Alzheimer's disease patients. Acta Neuropathol 1996; 91: 6-14.

Fiala M, Liu QN, Sayre J, Pop V, Brahmandam V, Graves MC et al. Cyclooxygenase-2-positive macrophages infiltrate the Alzheimer's disease brain and damage the blood-brain barrier. Eur J Clin Invest 2002; 32: 360-371.

Kawai M, Kalaria RN, Harik SI, Perry G. The relationship of amyloid plaques to cerebral capillaries in Alzheimer's disease. Am J Pathol 1990; 137: 1435-1446.

Jonker C, Eikelenboom P, Tavenier P. Immunological indices in the cerebrospinal fluid of patients with presenile dementia of the Alzheimer type. Br J Psychiatry 1982; 140: 44-49.

Ryu JK, McLarnon JG. A leaky blood-brain barrier, fibrinogen infiltration and microglial reactivity in inflamed Alzheimer's disease brain. J Cell Mol Med 2009; 13: 2911-2925.

Stewart PA, Hayakawa K, Akers MA, Vinters HV. A morphometric study of the blood-brain barrier in Alzheimer's disease. Lab Invest 1992; 67: 734-742.

Tomimoto $\mathrm{H}$, Akiguchi I, Suenaga T, Nishimura M, Wakita $\mathrm{H}$, Nakamura $\mathrm{S}$ et al. Alterations of the blood-brain barrier and glial cells in white-matter lesions in cerebrovascular and Alzheimer's disease patients. Stroke 1996; 27: 2069-2074.

Ujiie M, Dickstein DL, Carlow DA, Jefferies WA. Blood-brain barrier permeability precedes senile plaque formation in an Alzheimer disease model. Microcirculation 2003; 10: 463-470.

Zipser BD, Johanson CE, Gonzalez L, Berzin TM, Tavares R, Hulette CM et al. Microvascular injury and blood-brain barrier leakage in Alzheimer's disease. Neurobiol Aging 2007; 28: 977-986.

Alafuzoff I, Adolfsson R, Grundke-Iqbal I, Winblad B. Blood-brain barrier in Alzheimer dementia and in non-demented elderly. An immunocytochemical study. Acta Neuropathol 1987; 73: 160-166.

Munoz DG, Erkinjuntti T, Gaytan-Garcia S, Hachinski V. Serum protein leakage in Alzheimer's disease revisited. Ann N Y Acad Sci 1997; 826: 173-189.

Poduslo JF, Curran GL, Wengenack TM, Malester B, Duff K. Permeability of proteins at the bloodbrain barrier in the normal adult mouse and double transgenic mouse model of Alzheimer's disease. Neurobiol Dis 2001; 8: 555-567.

Rozemuller JM, Eikelenboom P, Kamphorst W, Stam FC. Lack of evidence for dysfunction of the blood-brain barrier in Alzheimer's disease: an immunohistochemical study. Neurobiol Aging 1988; 9: 383-391.

Gunzel D, Yu ASL. Claudins and the Modulation of Tight Junction Permeability. Physiol Rev 2013; 93 : 525-569.

Romanitan MO, Popescu BO, Spulber S, Băjenaru O, Popescu LM, Winblad B et al. Altered expression of claudin family proteins in Alzheimer's disease and vascular dementia brains. J Cell Mol Med 2010; 14: 1088-1100.

Spulber S, Bogdanovic N, Romanitan MO, Bajenaru O a., Popescu BO. Claudin expression profile separates Alzheimer's disease cases from normal aging and from vascular dementia cases. J Neurol Sci 2012; 322: 184-186.

Farrall AJ, Wardlaw JM. Blood-brain barrier: Ageing and microvascular disease - systematic review and meta-analysis. Neurobiol Aging 2009; 30: 337-352.

Götz J, Ittner LM. Animal models of Alzheimer's disease and frontotemporal dementia. Nat Rev Neurosci 2008; 9: 532-544.

Garcia-Alloza M, Robbins EM, Zhang-Nunes SX, Purcell SM, Betensky R a., Raju S et al. 
Characterization of amyloid deposition in the APPswe/PS1dE9 mouse model of Alzheimer disease. Neurobiol Dis 2006; 24: 516-524.

Wunder A, Schoknecht K, Stanimirovic DB, Prager O, Chassidim Y. Imaging blood-brain barrier dysfunction in animal disease models. Epilepsia 2012; 53 Suppl 6: 14-21.

Blennow K, Fredman P, Wallin a, Gottfries CG, Karlsson I, Långström G et al. Protein analysis in cerebrospinal fluid. II. Reference values derived from healthy individuals 18-88 years of age. Eur Neurol 1993; 33: 129-133.

Tibbling G, Link H, Ohman S. Principles of albumin and IgG analyses in neurological disorders. I. Establishment of reference values. Scand J Clin Lab Invest 1977; 37: 385-390.

Algotsson A, Winblad B. The integrity of the blood-brain barrier in Alzheimer's disease. Acta Neurol Scand 2007; 115: 403-408.

Bowman GL, Kaye J a., Quinn JF. Dyslipidemia and blood-brain barrier integrity in Alzheimer's disease. Curr Gerontol Geriatr Res 2012; 2012: 184042.

Elovaara I, Icén A, Palo J, Erkinjuntti T. CSF in Alzheimer's disease. Studies on blood-brain barrier function and intrathecal protein synthesis. J Neurol Sci 1985; 70: 73-80.

Elovaara I, Palo J, Erkinjuntti T, Sulkava R. Serum and cerebrospinal fluid proteins and the bloodbrain barrier in Alzheimer's disease and multi-infarct dementia. Eur Neurol 1987; 26: 229-234.

Frölich L, Kornhuber J, Ihl R, Fritze J, Maurer K, Riederer P. Integrity of the blood-CSF barrier in dementia of Alzheimer type: CSF/serum ratios of albumin and IgG. Eur Arch Psychiatry Clin Neurosci 1991; 240: 363-366.

Skoog I, Wallin A, Fredman P, Hesse C, Aevarsson O, Karlsson I et al. A population study on bloodbrain barrier function in 85-year-olds: Relation to Alzheimer's disease and vascular dementia. Neurology 1998; 50: 966-971.

Wada H. Blood-brain barrier permeability of the demented elderly as studied by cerebrospinal fluidserum albumin ratio. Intern Med 1998; 37: 509-513.

Matsumoto Y, Yanase D, Noguchi-Shinohara M, Ono K, Yoshita M, Yamada M. Blood-brain barrier permeability correlates with medial temporal lobe atrophy but not with amyloid-beta protein transport across the blood-brain barrier in Alzheimer's disease. Dement Geriatr Cogn Disord 2007; 23: $241-245$.

Blennow K, Wallin a, Fredman P, Karlsson I, Gottfries CG, Svennerholm L. Blood-brain barrier disturbance in patients with Alzheimer's disease is related to vascular factors. Acta Neurol Scand 1990; 81: 323-326.

Mecocci P, Parnetti L, Reboldi GP, Santucci C, Gaiti a, Ferri C et al. Blood-brain-barrier in a geriatric population: barrier function in degenerative and vascular dementias. Acta Neurol Scand 1991; 84: 210-213.

Wallin A, Blennow K, Fredman P, Gottfries CG, Karlsson I, Svenner-holm L et al. Blood brain barrier function in vascular dementia. Acta Neurol Scand 1990; 81: 318-322.

Kay AD, May C, Papadopoulos NM, Costello R, Atack JR, Luxenberg JS et al. CSF and serum concentrations of albumin and IgG in Alzheimer's disease. Neurobiol Aging 1987; 8: 21-25.

Mattila KM, Pirttilä T, Blennow K, Wallin A, Viitanen M, Frey H. Altered blood-brain-barrier function in Alzheimer's disease? Acta Neurol Scand 1994; 89: 192-198. 
Dysken MW, Nelson MJ, Hoover KM, Kuskowski M, McGeachie R. Rapid dynamic CT scanning in primary degenerative dementia and age-matched controls. Biol Psychiatry 1990; 28: 425-434.

Caserta MT, Caccioppo D, Lapin GD, Ragin A, Groothuis DR. Blood-brain barrier integrity in Alzheimer's disease patients and elderly control subjects. J Neuropsychiatry Clin Neurosci 1998; 10: 78-84.

Hanyu H, Asano T, Tanaka Y, Iwamoto T, Takasaki M, Abe K. Increased blood-brain barrier permeability in white matter lesions of Binswanger's disease evaluated by contrast-enhanced MRI. Dement Geriatr Cogn Disord 2002; 14: 1-6.

Schlageter NL, Carson RE, Rapoport SI. Examination of blood-brain barrier permeability in dementia of the Alzheimer type with [68Ga]EDTA and positron emission tomography. J Cereb Blood Flow Metab 1987; 7: 1-8.

Starr JM, Farrall AJ, Armitage P, McGurn B, Wardlaw JM. Blood-brain barrier permeability in Alzheimer's disease: a case-control MRI study. Psychiatry Res - Neuroimaging 2009; 171: 232-241.

2

Taheri S, Gasparovic C, Shah NJ, Rosenberg G a. Quantitative measurement of blood-brain barrier permeability in human using dynamic contrast-enhanced MRI with fast T1 mapping. Magn Reson Med 2011; 65: 1036-1042.

Taheri S, Gasparovic C, Huisa BN, Adair JC, Edmonds E, Prestopnik J et al. Blood-brain barrier permeability abnormalities in vascular cognitive impairment. Stroke 2011; 42: 2158-2163.

Wang H, Golob EJ, Su MY. Vascular volume and blood-brain barrier permeability measured by dynamic contrast enhanced MRI in hippocampus and cerebellum of patients with $\mathrm{MCl}$ and normal controls. J Magn Reson Imaging 2006; 24: 695-700.

5

Van Assema DME, Lubberink M, Bauer M, Van Der Flier WM, Schuit RC, Windhorst AD et al. Bloodbrain barrier P-glycoprotein function in Alzheimer's disease. Brain 2012; 135: 181-189.

van Assema DM, Goos JD, van der Flier WM, Lubberink M, Boellaard R, Windhorst AD et al. No evidence for additional blood-brain barrier P-glycoprotein dysfunction in Alzheimer's disease patients with microbleeds. J Cereb Blood Flow Metab 2012; 32: 1468-1471.

Sourbron SP, Buckley DL. Classic models for dynamic contrast-enhanced MRI. NMR Biomed 2013; 26: 1004-1027.

Patlak CS, Blasberg RG, Fenstermacher JD. Graphical evaluation of blood-to-brain transfer constants from multiple-time uptake data. J Cereb Blood Flow Metab 1983; 3: 1-7.

Nitta T, Hata M, Gotoh S, Seo Y, Sasaki H, Hashimoto N et al. Size-selective loosening of the bloodbrain barrier in claudin-5-deficient mice. J Cell Biol 2003; 161: 653-660.

Thomsen HS, Morcos SK, Almén T, Bellin MF, Bertolotto M, Bongartz G et al. Nephrogenic systemic fibrosis and gadolinium-based contrast media: Updated ESUR Contrast Medium Safety Committee guidelines. Eur Radiol 2013; 23: 307-318.

Babikian V, Ropper a H. Binswanger's disease: a review. Stroke 1987; 18: 2-12.

Minoshima S, Giordani B, Berent S, Frey K a, Foster NL, Kuhl DE. Metabolic reduction in the posterior cingulate cortex in very early Alzheimer's disease. Ann Neurol 1997; 42: 85-94.

Deane R, Bell RD, Sagare A, Zlokovic BV. Clearance of amyloid-beta peptide across the blood-brain barrier: implication for therapies in Alzheimer's disease. CNS Neurol Disord Drug Targets 2009; 8: 16-30.

Didziapetris R, Japertas P, Avdeef A, Petrauskas A. Classification analysis of P-glycoprotein substrate specificity. J Drug Target 2003; 11: 391-406. 
Kessler RM, Goble JC, Bird JH, Girton ME, Doppman JL, Rapoport SI et al. Measurement of BloodBrain Barrier Permeability with Positron Emission Tomography and [68Ga]EDTA. J Cereb Blood Flow Metab 1984; 4: 323-328.

Agdeppa ED, Spilker ME. A review of imaging agent development. AAPS J 2009; 11: 286-299.

Umutlu L, Maderwald S, Kraff O, Theysohn JM, Kuemmel S, Hauth E a. et al. Dynamic ContrastEnhanced Breast MRI at 7 Tesla Utilizing a Single-loop Coil. A Feasibility Trial. Acad Radiol 2010; 17: 1050-1056.

Umutlu L, Kraff O, Orzada S, Fischer A, Kinner S, Maderwald S et al. Dynamic contrast-enhanced renal MRI at 7 Tesla: preliminary results. Invest Radiol 2011; 46: 425-433.

Tofts PS, Brix G, Buckley DL, Evelhoch JL, Henderson E, Knopp M V. et al. Estimating kinetic parameters from dynamic contrast-enhanced T1- weighted MRI of a diffusable tracer: Standardized quantities and symbols. J Magn Reson Imaging 1999; 10: 223-232.

Schabel MC, Parker DL. Uncertainty and bias in contrast concentration measurements using spoiled gradient echo pulse sequences. Phys Med Biol 2008; 53: 2345-2373.

Dyrna F, Hanske S, Krueger M, Bechmann I. The blood-brain barrier. J Neuroimmune Pharmacol 2013; 8: 763-773.

Kimelberg HK. Water homeostasis in the brain: Basic concepts. Neuroscience 2004; 129: 851-860.

Le Bihan D, Breton E, Lallemand D, Grenier P, Cabanis E, Laval-Jeantet M. MR imaging of intravoxel incoherent motions: application to diffusion and perfusion in neurologic disorders. Radiology 1986; 161: 401-407.

Hales PW, Clark CA. Combined arterial spin labeling and diffusion-weighted imaging for noninvasive estimation of capillary volume fraction and permeability-surface product in the human brain. J Cereb Blood Flow Metab 2012; 33: 67-75.

Agyare EK, Leonard SR, Curran GL, Yu CC, Lowe VJ, Paravastu AK et al. Traffic Jam at the Blood-Brain Barrier Promotes Greater Accumulation of Alzheimer's Disease Amyloid- $\beta$ Proteins in the Cerebral Vasculature. Mol Pharm 2013; 10: 1557-1565..

Klunk WE, Engler H, Nordberg A, Wang Y, Blomqvist G, Holt DP et al. Imaging Brain Amyloid in Alzheimer's Disease with Pittsburgh Compound-B. Ann Neurol 2004; 55: 306-319.

Small GW, Kepe V, Ercoli LM, Siddarth P, Bookheimer SY, Miller KJ et al. PET of brain amyloid and tau in mild cognitive impairment. N Engl J Med 2006; 355: 2652-2663.

Echávarri C, Burgmans S, Caballero MC, García-Bragado F, Verhey FRJ, Uylings HBM. Co-occurrence of different pathologies in dementia: Implications for dementia diagnosis. J Alzheimer's Dis 2012; 30: 909-917.

Jack CR, Knopman DS, Jagust WJ, Shaw LM, Aisen PS, Weiner MW et al. Hypothetical model of dynamic biomarkers of the Alzheimer's pathological cascade. Lancet Neurol 2010; 9: 119-128.

Tabert MH, Manly JJ, Liu X, Pelton GH, Rosenblum S, Jacobs M et al. Neuropsychological prediction of conversion to Alzheimer disease in patients with mild cognitive impairment. Arch Gen Psychiatry 2006; 63: 916-924.

91 Choi SR, Schneider JA, Bennett DA, Beach TG, Bedell BJ, Zehntner SP et al. Correlation of Amyloid PET Ligand Florbetapir F 18 Binding With $A \beta$ Aggregation and Neuritic Plaque Deposition in Postmortem Brain Tissue. Alzheimer Dis Assoc Disord 2012; 26: 8-16. 
93 Leonardi a, Gandolfo C, Caponnetto C, Arata L, Vecchia R. The integrity of the blood-brain barrier in Alzheimer's type and multi-infarct dementia evaluated by the study of albumin and IgG in serum and cerebrospinal fluid. J Neurol Sci 1985; 67: 253-261.

94 Matsumoto Y, Yanase D, Noguchi-Shinohara M, Ono K, Yoshita M, Yamada M. Cerebrospinal fluid/serum IgG index is correlated with medial temporal lobe atrophy in Alzheimer's disease. Dement Geriatr Cogn Disord 2008; 25: 144-147.

95 Bowman GL, Kaye JA, Moore M, Waichunas D, Carlson NE, Quinn JF. Blood-brain barrier impairment in Alzheimer disease: Stability and functional significance. Neurology 2007; 68: 1809-1814. 



\section{Chapter 3}

\section{Blood-brain barrier leakage in early Alzheimer's disease}

Harm J van de Haar, Saartje Burgmans, Jacobus FA Jansen, Matthias JP van Osch, Mark A van Buchem, Majon Muller, Paul AM Hofman, Frans RJ Verhey, Walter H Backes 


\section{Abstract}

Increased blood-brain barrier (BBB) permeability may represent a key mechanism in the early pathology of Alzheimer's disease (AD). Our aim was to investigate whether the BBB is leaking blood-circulating substances in patients with early forms of $A D$, and if so, to examine the extent and pattern of leakage. As a pilot study, we scanned 16 patients with early $A D$ and 17 healthy age matched controls using a dual time resolution dynamic contrast-enhanced MRI sequence for 25 minutes. The Patlak graphical approach was used to quantify BBB leakage rate $\left(\mathrm{K}_{\mathrm{i}}\right)$ and local blood plasma volume $\left(\mathrm{V}_{\mathrm{p}}\right)$. Subsequent histogram analysis was used to determine the volume fraction of the leaking brain tissue $(\mathrm{V} L)$. Differences were assessed using linear regression analysis, adjusted for confounding variables. $K_{i}$ was significantly higher in patients compared with controls in the total GM $(p<0.05)$, and cortex $(p=0.03)$. Patients had a significantly higher $V_{L}$ in the $G M(p=0.004)$, NAWM $(p<0.04)$, deep GM $(p=0.01)$, and cortex $(p=0.004)$, and a trend in WM $(p=0.06)$. Over all subjects, the MMSE score decreased significantly with increasing $\mathrm{VL}$ in the deep $G M(p=0.007)$, and cortex $(p<0.050)$. This study demonstrates global BBB leakage in patients with early $A D$ that is associated with cognitive decline. We suggest that $a$ compromised BBB is part of a cascade of pathological events, eventually leading to cognitive decline and dementia. 


\section{Introduction}

Evidence is increasing that cerebral microvasculature impairment plays an important role in the pathophysiology of Alzheimer's disease $(A D)^{1,2}$. However, the exact pathway remains unclear. Results from histology and albumin sampling studies show that an increased blood-brain barrier (BBB) permeability is likely a key mechanism³.

The BBB is a collection of cells and subcellular structures in the cerebrovascular wall, which separates the blood from the brain parenchyma. It regulates the delivery of important nutrients to the brain through active and passive transport mechanisms, and prevents neurotoxins from entering the brain ${ }^{4}$. It also has a clearance function, meaning that it removes surplus substances from the brain. A well-functioning BBB is essential to keep the brain tissue in a healthy condition. Previous studies suggest that deterioration of the BBB can cause an ill-conditioned environment for neuronal cells and other pathological changes, such as small vessel pathology, protein deposits, inflammation and neuronal cell death ${ }^{5,6}$. These changes may eventually lead to cognitive decline and dementia.

BBB degradation in advanced stages $A D$ has been shown mainly by using histology and the albumin ratio measurement ${ }^{7}$. For the detection of subtle BBB leakage in vivo, dynamic contrast-enhanced magnetic resonance imaging (DCE-MRI) can be used, which is a well-established method in (neuro)oncology due to relatively strong leakage of gadolinium contrast agent in (high-grade) tumors ${ }^{8}$. Using longer scan times, it is also becoming a promising method in neurodegenerative and cerebrovascular diseases, where the leakage is expected to be much lower ${ }^{9-12}$.

To investigate whether BBB leakage contributes to the early pathophysiology of $A D$, we hypothesized that patients with early forms of $A D$ will already show increased $B B B$ permeability in comparison with age matched controls. For this, we initiated a pilot study that used a dedicated dual time resolution MRI acquisition protocol that separates the filling of the blood vessels from the leakage ${ }^{13}$. We also investigated differences in local blood plasma volume fraction, and the relationship between BBB permeability and global cognition. 


\section{Materials and Methods}

\section{Participants}

Patients with $\mathrm{MCl}$ due to $A D$ or patients at an early phase of $A D$ were prospectively recruited at the memory clinic from the Maastricht University Medical Center (MUMC) and Leiden University Medical Center (LUMC). Since MCI due to $A D$ and $A D$ comprise a continuum of cognitive decline, both groups were combined for our primary analysis. All patients were referred by their general practitioner for memory complaints. They were diagnosed at each center in consensus by a multidisciplinary team according to the Dubois criteria for $\mathrm{MCl}$ and the criteria of the National Institute on Aging and the Alzheimer's Association for $A D^{14,15}$. Patients were included when they were diagnosed with either dementia of the Alzheimer type or mild cognitive impairment due to AD. To ensure the patients had an early stage of $A D$, the clinical dementia rating was $\leq 1^{16}$. Healthy controls were recruited through advertisements in local newspapers. Participants were excluded in the following conditions: contraindications for scanning (e.g. brain surgery, cardiac pacemaker, metal implants, claustrophobia, large body tattoos); contraindications for contrast agent (renal failure) as determined by the estimated Glomular Filtration Rate (eGFR) $<30 \mathrm{~mL} / \mathrm{min}$, or known allergy to gadolinium based contrast agents; major vascular disorders (e.g. stroke, heart disease) history of stroke or other causes for vascular dementia; psychiatric disorders: history of major depression, schizophrenia, bipolar disorder, psychotic disorder NOS or treatment for a psychotic disorder (< 12 months); epilepsy; Parkinson's disease; MS; brain surgery; brain trauma; electroshock therapy; kidney dialysis; Ménière's disease; brain infections; major structural abnormalities of the brain; cognitive impairment due to alcohol/drug abuse or abuse of other substances; absence of reliable informant (for patients). An overview of the results of neuropsychological tests of the patients can be found in Table A3.1 in the Appendix. This study was approved by the local medical ethical committees of both institutes. Written informed consent was obtained from all subjects. All subjects underwent the mini mental state examination (MMSE) test before MRI ${ }^{17}$. The patients had a score $\leq 2$ on the modified Hachinski scale, indicating that the dementia did not have a vascular origin. We included 18 patients and 19 healthy controls ( 5 and 4 at the LUMC, respectively). Two patients were excluded because of incomplete MRI examinations and 2 controls were excluded because of severe motion induced artifacts and a low renal function. Therefore, the data of 16 patients $(7 \mathrm{AD}, 9 \mathrm{MCl})$ and 17 healthy 
Table 3.2: Subject characteristics

\begin{tabular}{llll} 
Mean (sd) or \% & Patients & Healthy controls & $\mathrm{p}$ \\
\hline Number & $16(7 \mathrm{AD}$, & 17 & $\ldots$ \\
& $9 \mathrm{MCl})$ & & \\
Age (y) & $73.6(7.9)$ & $75.8(6.2)$ & 0.4 \\
range & $59-85$ & $65-85$ & \\
Sex (\% women) & 44 & 35 & 0.6 \\
Age of men (y) & $71.0(6.7)$ & $74.6(5.6)$ & 0.2 \\
range & $59-81$ & $65-85$ & \\
Age of women (y) & $76.9(8.6)$ & $78.2(6.9)$ & 0.8 \\
range & $64-85$ & $66-86$ & \\
MMSE & $26.3(1.9)$ & $29.5(0.6)$ & $<0.001$ \\
White matter hyperintensity volume (cm $\left.{ }^{3}\right)$ & $15.8(15.8)$ & $7.8(11.9)$ & 0.11 \\
Fazekas & $1.8(0.9)$ & $1.4(0.9)$ & 0.16 \\
MTA (average I and $\mathrm{r}$ ) & $1.6(1)$ & $0.6(0.7)$ & 0.003 \\
Diabetes (\%) & 38 & 6 & 0.03 \\
Hypertension (\%) & 56 & 53 & 0.8 \\
Other vascular diseases† (\%) & 31 & 18 & 0.4 \\
\hline
\end{tabular}

MMSE: mini-mental state examination. MTA: medial temporal lobe atrophy. $\dagger$ : includes cardiac arrhythmia, coronary disease and atherosclerosis.

controls was used for final analysis. Participant characteristics are provided in Table 3.1 .

\section{Imaging protocol}

To detect BBB leakage, a dual time resolution dynamic contrast-enhanced MRI protocol was implemented on a 3 Tesla MRI system (Philips Achieva, Best, the Netherlands) at both sites. This protocol consisted of two nested pulse sequences, a slow and a fast sequence ${ }^{13}$. The fast sequence was a saturation recovery gradient recalled sequence (TR/TE 5.2/2.5 ms, flip angle $30^{\circ}, 25.6 \times 20 \times 5 \mathrm{~cm}^{3}$ field of view (FOV), $256 \times 200 \times 10$ matrix, dynamic scan interval (DSI) $3.2 \mathrm{~s}$ ) with a saturation prepulse given at a delay time (TD) of $120 \mathrm{~ms}$. It was used during bolus injection (gadobutrol, $0.1 \mathrm{mmol} / \mathrm{kg}$, injected using a power injector with a flow of $3 \mathrm{ml} / \mathrm{s}$, followed by a $20 \mathrm{ml}$ saline flush) for 1.5 minutes. The slow sequence was a saturation recovery gradient recalled sequence (TR/TE 5.6/2.5 ms, $25.6 \times 25.6 \times 10 \mathrm{~cm}^{3} \mathrm{FOV}, 256 \times 256 \times 50$ matrix, DSI $30.5 \mathrm{~s}$ ) 
with the same prepulse. The slow sequence started immediately after the fast sequence, for a total time of 25 minutes. In the dual time resolution scan, the fast sequence allowed for a higher temporal resolution during initial arrival and recirculation of the contrast agent, while the slow sequence provided a higher spatial, but lower temporal resolution during contrast agent distribution and washout (Figure 3.1). To include most white matter hyperintensities (WMHs), the centers of the field of view in the fast and slow sequences were colocalized just under the genu of the corpus callosum. Additional information on the imaging protocol can also be found in the Appendix.

\section{MRI analysis}

Individual vascular input functions (VIF) were extracted from the superior sagittal sinus using an automated method ${ }^{18}$. The sagittal sinus was chosen as this was the largest cerebral blood vessel in the FOV, and has been used in other studies ${ }^{13,19,20}$. A twocompartment pharmacokinetic model was applied per voxel using the Patlak graphical approach $^{21}$, which was found to be the most appropriate model in a low-leakage regime 22,23 . The Patlak graphical approach provided the BBB leakage rate $K_{i}$ and the local blood plasma volume $v_{p}$. The Patlak graphical approach is based on linear fits of scatterplots. The slope of this fit is the blood-brain barrier (BBB) leakage rate $\mathrm{K}_{\mathrm{i}}$ (assuming a tissue density of $1 \mathrm{~g} / \mathrm{ml}$ ), and the intercept is the local blood plasma volume $v_{p}$. However, the scatterplots were rather noisy due to the very low permeability of brain tissue, thus yielding not only positive, but also negative slope values. To further increase the sensitivity of this leakage detection, a histogram approach was used. The histograms were normalized and noise was estimated by assuming that negative slope values can only be attributed to noise, and that a similar distribution of noise is also present in positive slope values. The data were then corrected by subtracting the estimated noise from the measured histogram. The remaining cumulative sum of the bins was defined as the BBB leakage volume fraction VL (Figure 3.2). 


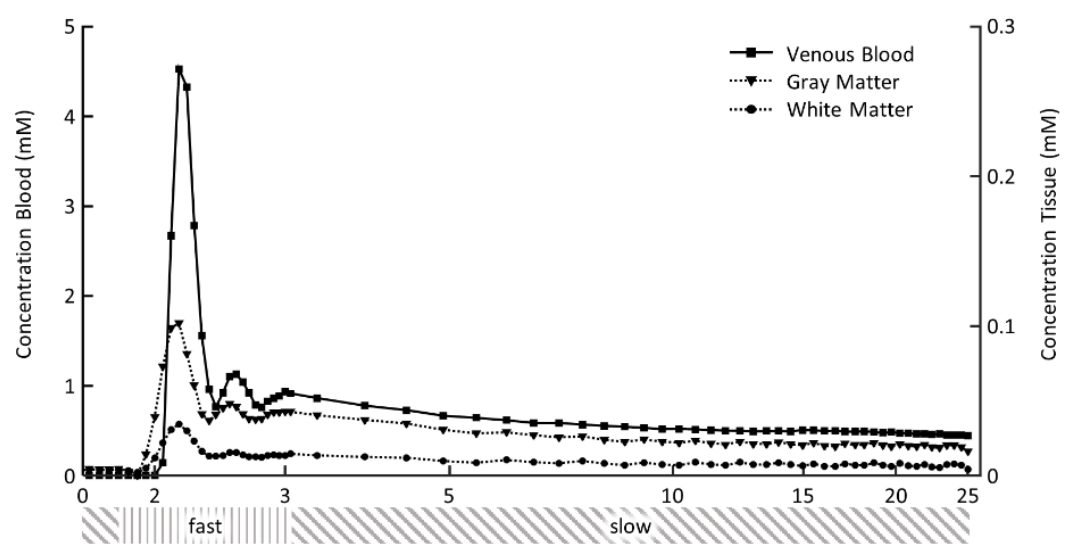

Time (min)

Figure 3.1: Example curves of the contrast agent concentration in venous blood used as a vascular input function, and the entire normal appearing white matter and the total gray matter (deep and cortex) of a single subject (male, age $75 \mathrm{y}$ ). The time axis is on a logarithmic scale to emphasize the rapid changes during contrast agent arrival and initial recirculations (moment of injection \pm 1:45 minutes). Below the time axis is a bar depicting when the fast and slow temporal resolution scan part was running.

A

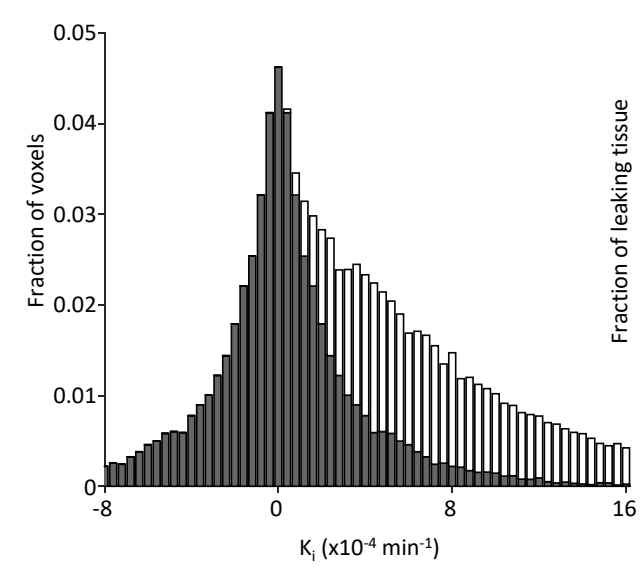

B

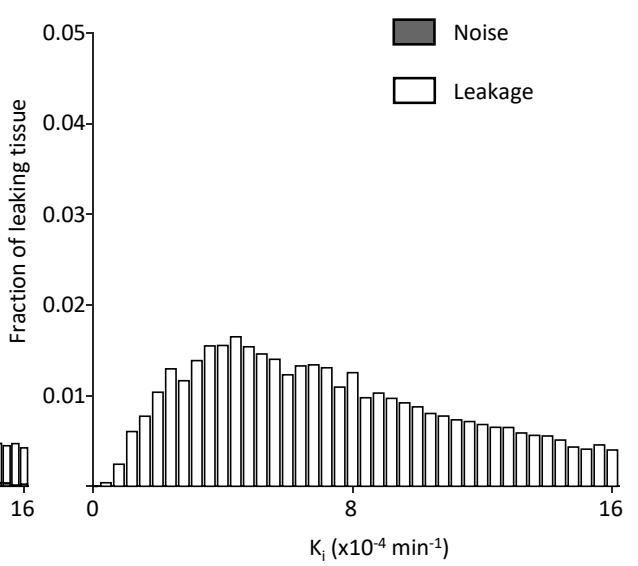

Figure 3.2: Overview of the noise estimation method. The noise estimation method is based on the bins with negative slope values, which are considered as noise. By assuming the same distribution on the positive side, the noise (dark gray bins in A) can be discerned from the detectable leakage (white bins in A). By subtracting the noise from the original histogram, the fraction of leaking tissue $\left(V_{L}\right)$ is obtained $(B)$. 


\section{Statistical analysis}

All statistical analyses were performed using the Statistical Package for the Social Sciences (IBM Corp. Released 2011. IBM SPSS Statistics for Windows, Version 20.0, NY, United States). Group characteristics were compared using chi-squared (categorical variables) and independent-samples two-sided $t$-tests (continuous variables). Differences in the median $K_{i}$ and $v_{p}$ values between the patients and controls were tested with an independent-samples two-sided $t$-test.

The (uncorrected) differences in $\mathrm{VL}$ between the groups was first tested using an independent-samples two-sided $t$-test. Next, linear regression was used to correct for age, sex, relative WMH volume (except when testing BBB leakage in the WMHs), diabetes status, and other non-cerebral vascular disease incidence. We also used linear regression to investigate the association between BBB leakage and MMSE score in the different tissue classes, corrected for age, sex and relative WMH volume. All tests were performed in two steps: first the total white matter (WM) and total gray matter (GM) were investigated, which was then divided into normal appearing white matter (NAWM), deep $\mathrm{GM}$, cortex and WMH. A false discovery rate (FDR) procedure $(\mathrm{q}=0.05)$ was performed to correct for multiple comparisons over various brain tissue regions ${ }^{24}$.

We also did a post-hoc analysis of patients with $\mathrm{MCl}$ and $\mathrm{AD}$ separately, by comparing the BBB leakage fraction using independent-samples two-sided $t$-tests between the three groups. Statistical significance was inferred when $p<0.05$.

\section{Results}

The patients had a significantly lower MMSE score and were significantly more often diagnosed with diabetes than the controls. A chi-squared test revealed no significant difference in center enrollment $(p=0.9)$.

Examples of the obtained concentration time curves are displayed in Figure 3.1 and examples of representative $K_{i}$ maps are displayed in Figures 3.3 and 3.4. There was no detectable leakage in the cerebrospinal fluid (CSF) of the lateral ventricles. An overview of the grouped histograms from which VL was calculated in the WM and GM is displayed in Figure 3.5. 


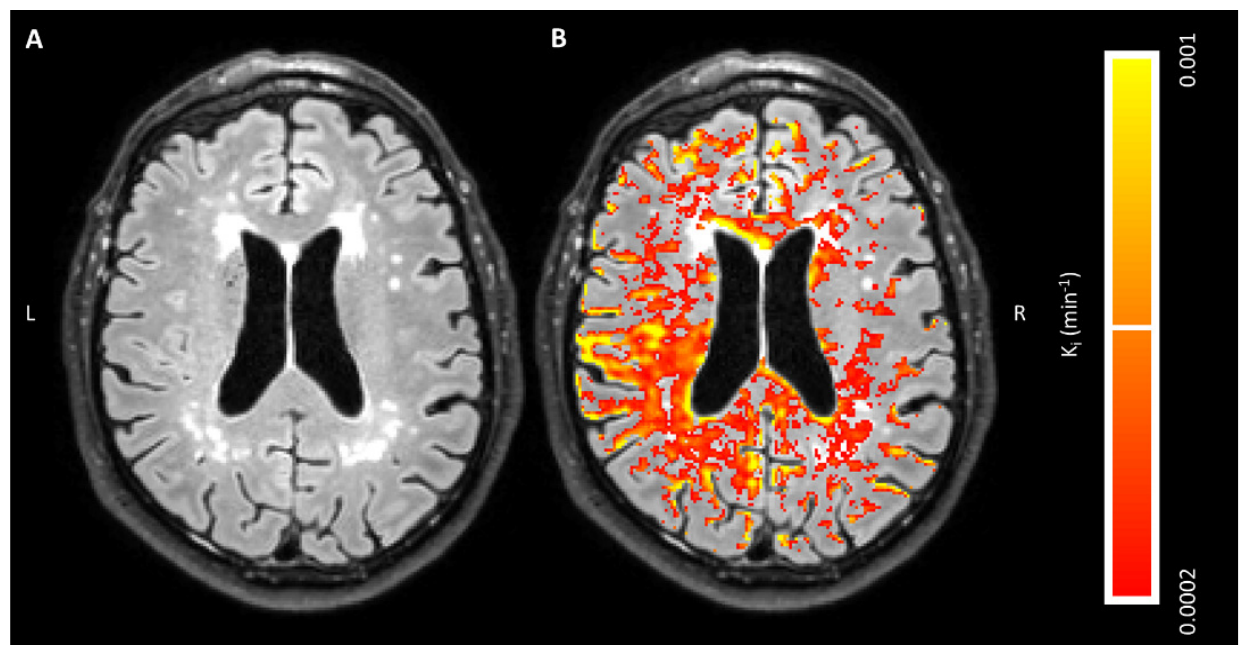

Figure 3.3: Example of a FLAIR image (axial, A) of a patient (male, age $68 \mathrm{y}$ ) with the corresponding $K_{i}$ maps superimposed (B). $K_{i}$ values appear diffusely distributed in both subjects, with some periventricular hotspots. The leakage manifests in the normal appearing white matter, white matter hyperintensities as well as gray matter. Voxels with a low signal-to-noise ratio in the MRI signal intensity were removed, and the $\mathrm{K}_{\mathrm{i}}$ map was masked to the cerebrum.

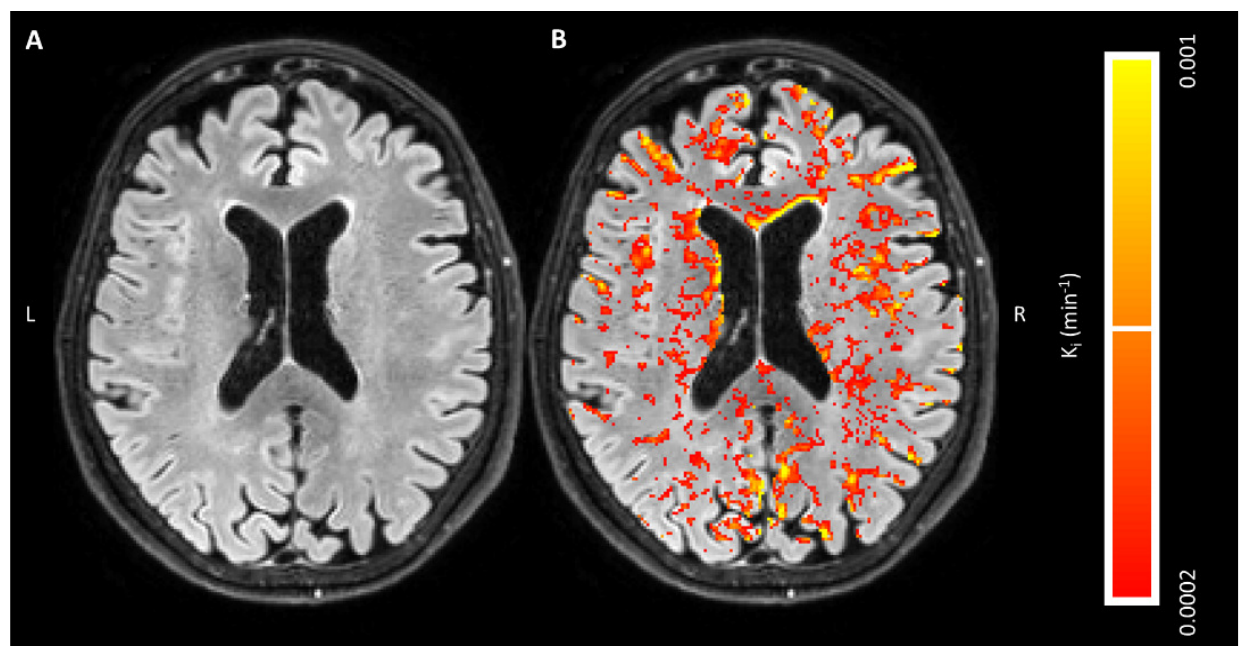

Figure 3.4: Example of a FLAIR image (axial, $A$ ) of a healthy control (B, female, age $80 \mathrm{y}$ ) with the corresponding Ki maps superimposed (B). Voxels with a low signal-to-noise ratio in the MRI signal intensity were removed, and the Ki map was masked to the cerebrum. 
A

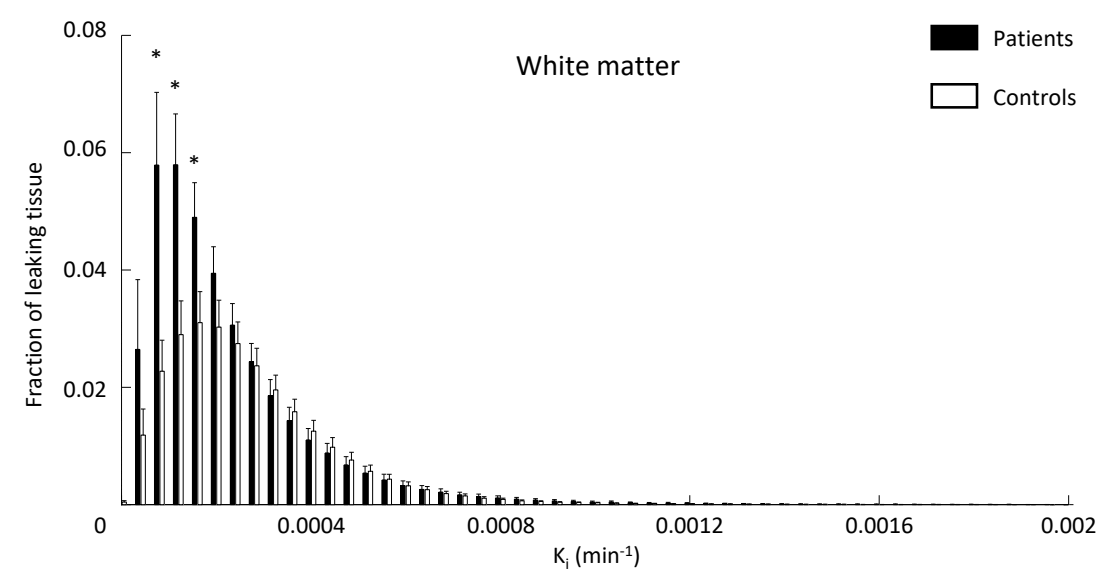

B

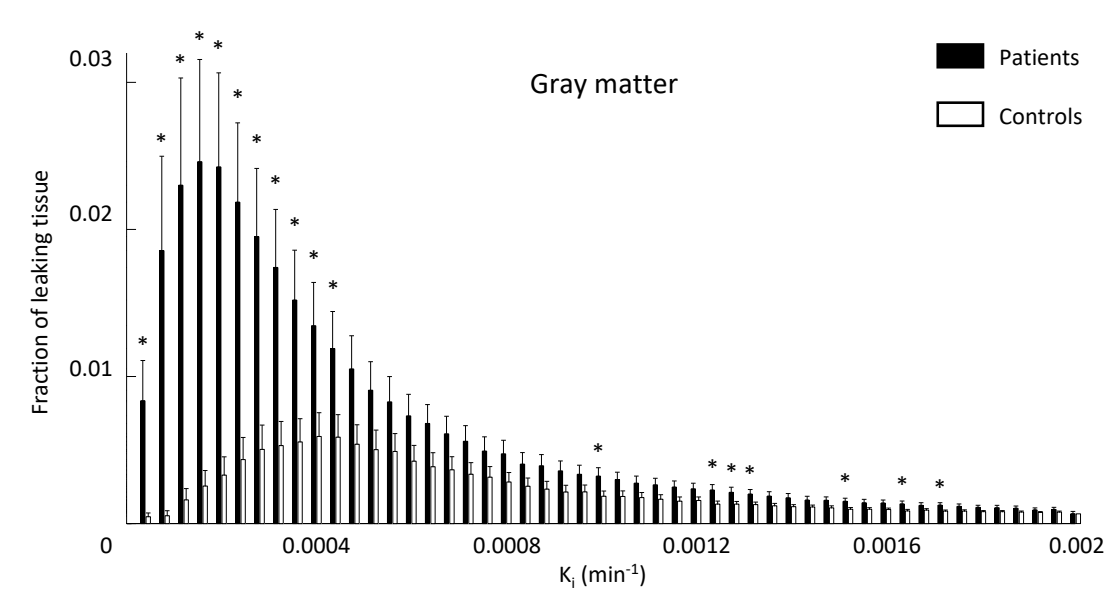

Figure 3.5: Histograms of the fraction of leaking voxels for the patients and controls in the white matter $(A)$ and gray matter $(B)$. Bins containing noise have been removed, so only the bins representing actual leakage are displayed. Note that the patients have a larger proportion of leaking voxels compared with controls. The individual bins were statistically tested $\left({ }^{*} p<0.05\right)$ to illustrate that the nature of the difference between the groups is particularly in the low $\mathrm{K}_{\mathrm{i}}$ range (these tests were not used to draw any further conclusions). The cumulative sum of the bins, $\mathrm{V}_{\mathrm{L}}$, represents a summary measure that is more sensitive than the median $\mathrm{K}_{\mathrm{i}}$. 
Table 3.2: Regional comparison of DCE-MRI measures

\begin{tabular}{|c|c|c|c|c|c|c|c|c|c|}
\hline \multirow[b]{2}{*}{ ROI } & \multicolumn{3}{|c|}{$\begin{array}{l}\text { Leakage rate } \\
\left(\mathrm{K}_{\mathrm{i}} \times 10^{-4} \mathrm{~min}^{-1}\right)\end{array}$} & \multicolumn{3}{|c|}{$\begin{array}{l}\text { Fraction of leaking tissue } \\
\left(v_{L}\right)\end{array}$} & \multicolumn{3}{|c|}{$\begin{array}{l}\text { Fractional blood plasma } \\
\text { volume } \\
\left(v_{p} \times 10^{-2}\right)\end{array}$} \\
\hline & Patients & Controls & $\mathrm{p}$ & Patients & Controls & $p^{5}$ & Patients & Controls & $p$ \\
\hline WM & $\begin{array}{l}0.66 \pm \\
0.44\end{array}$ & $\begin{array}{l}0.70 \pm \\
0.64\end{array}$ & 0.8 & $\begin{array}{l}0.37 \pm \\
0.17\end{array}$ & $\begin{array}{l}0.57 \pm \\
0.14\end{array}$ & $0.019^{\dagger \neq}$ & $0.4 \pm 0.2$ & $0.6 \pm 0.1$ & $0.014^{\dagger}$ \\
\hline GM & $\begin{array}{l}0.89 \pm \\
1.12\end{array}$ & $\begin{array}{l}0.17 \pm \\
0.81\end{array}$ & $<0.05$ & $\begin{array}{l}0.32 \pm \\
0.23\end{array}$ & $\begin{array}{l}0.12 \pm \\
0.10\end{array}$ & $0.004^{\dagger \neq}$ & $1.1 \pm 0.4$ & $1.5 \pm 0.3$ & $<0.001^{\dagger}$ \\
\hline NAWM & $\begin{array}{l}0.65 \pm \\
0.43\end{array}$ & $\begin{array}{l}0.70 \pm \\
0.64\end{array}$ & 0.8 & $\begin{array}{l}0.38 \pm \\
0.16\end{array}$ & $\begin{array}{l}0.27 \pm \\
0.13\end{array}$ & 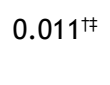 & $0.4 \pm 0.2$ & $0.6 \pm 0.1$ & $0.015^{\dagger}$ \\
\hline $\begin{array}{l}\text { Deep } \\
\text { GM }\end{array}$ & $\begin{array}{l}1.25 \pm \\
1.15\end{array}$ & $\begin{array}{l}0.84 \pm \\
1.41\end{array}$ & 0.4 & $\begin{array}{l}0.28 \pm \\
0.21\end{array}$ & $\begin{array}{l}0.12 \pm \\
0.11\end{array}$ & $0.004^{\dagger \neq}$ & $1.1 \pm 0.4$ & $1.4 \pm 0.2$ & $0.008^{\dagger}$ \\
\hline Cortex & $\begin{array}{l}0.84 \pm \\
1.14\end{array}$ & $\begin{array}{l}0.08 \pm \\
0.08\end{array}$ & 0.03 & $\begin{array}{l}0.31 \pm \\
0.23\end{array}$ & $\begin{array}{l}0.11 \pm \\
0.10\end{array}$ & $0.004^{\dagger \neq}$ & $1.1 \pm 0.4$ & $1.6 \pm 0.3$ & $<0.001^{\dagger}$ \\
\hline WMHs & $\begin{array}{l}1.06 \pm \\
1.11\end{array}$ & $\begin{array}{l}0.61 \pm \\
0.77\end{array}$ & 0.19 & $\begin{array}{l}0.29 \pm \\
0.18\end{array}$ & $\begin{array}{l}0.24 \pm \\
0.16\end{array}$ & 0.2 & $0.6 \pm 0.3$ & $0.9 \pm 0.3$ & $0.003^{\dagger}$ \\
\hline
\end{tabular}

All data (other than $\mathrm{p}$ values) are mean \pm one standard deviation. $\mathrm{ROI}=$ region of interest, $\mathrm{WM}=$ white matter, GM = gray matter, NAWM = normal appearing white matter, WMHs = white matter hyperintensities.

† significant after correction for multiple comparisons using the False Discovery Rate (FDR) approach ‡ significant after additional correction for diabetes and other non-cerebrovascular disease

$\S$ Adjusted for age, sex, and relative WMH volume

The results of the regional analysis of the various measures can be found in Table 3.2. In short, $\mathrm{K}_{\mathrm{i}}$ was significantly higher in patients compared with controls in the total $\mathrm{GM}$, and cortex, but not in the WM, NAWM, deep GM or WMHs (Table 3.2). Patients had a significantly higher $V_{L}$ in the GM $(p=0.004)$, NAWM $(p<0.04)$, deep GM $(p=0.01)$, and cortex $(p=0.004)$, a trend in WM $(p=0.06)$ but not in $W M H$. When adjusting for all covariates the patients exhibited a significantly higher $V_{L}$ in the $W M(B=0.136)$ and $G M$ $(B=0.214)$, and also in the NAWM $(B=0.139)$, deep $G M(B=0.182)$, cortex $(B=0.207)$, but not in WMHs $(B=0.075$, Table 3.2). Investigating the impact of diabetes and other noncerebral vascular diseases in the total model, provided comparable results with patients exhibiting a significantly higher $V L$ in the $W M(B=0.125, p<0.05)$ and $G M(B=0.2229$, $p=0.005)$, and also in the NAWM $(B=0.128, p=0.03)$, deep $G M(B=0.202, p=0.006)$, cortex 
$(B=0.216, p=0.006)$, but again not in WMHs $(B=0.092, p=0.2)$. Neither the presence of diabetes nor other vascular diseases was significant in any of these models. The median $v_{p}$ was significantly lower in the patients compared with the controls (Table 3.2) in all tissue classes. Over all subjects, the MMSE score decreased significantly with increasing $\mathrm{VL}$ in the deep GM $(B=-0.039, p=0.007$, and remained significant after FDR correction), and cortex $(B=-0.033, p<0.050)$, with a trend in GM $(B=-0.033, p=0.06)$ and NAWM $(B=-$ $0.022, p=0.09)$, but not significantly in WM $(p=0.1)$ or WMH $(p=0.3)$.

In the subset of patients with $\mathrm{MCl}$, VL was also higher than for controls in the deep GM $(p=0.02)$ and cortex $(p<0.05)$ with a trend in the GM $(p=0.05)$, WM $(p<0.1)$ and NAWM $(p=0.08)$, and also patients with $A D$ had a higher $v_{L}$ than the controls in the $G M(p=0.04)$ and cortex $(p=0.04)$, with a trend in deep $G M(p=0.07)$. There were no significant differences in other tissue types ( $p>0.1)$. When comparing $\mathrm{MCl}$ patients with $A D$ patients, no significant differences were found in any of the tissue classes investigated $(p>0.6)$.

\section{Discussion}

This study demonstrates increased BBB leakage in patients with early AD. The leakage was globally distributed throughout the cerebrum and associated with declined global cognitive performance. Using dual time resolution DCE-MRI, we found an increased $K_{i}$ in $G M$ for early $A D$. By also showing very subtle BBB impairment in the WM, VL proved to be even more sensitive to the differences in BBB leakage than the leakage rate $\mathrm{K}_{\mathrm{i}}$. Not only does this show that the differences between patients with early $A D$ and healthy controls are in the extent of the BBB leakage rather than the rate (i.e. strength), it also shows that the leakage is widespread rather than localized to a single tissue class such as WMH, NAWM or cortex. Additionally, the BBB impairment did not (fully) originate from vascular pathology, as adding diabetes and other non-cerebral vascular diseases to the analysis model did not change the results. This suggests that the BBB impairment stems from the $A D$ pathology instead of vascular comorbidities. Previously, only a few studies reported on BBB impairment in dementia using contrast-enhanced MRI. Starr et al. investigated the $B B B$ in patients with early $A D^{25}$. They found dynamic signal enhancement differences that suggest altered blood-brain-CSF compartment kinetics compared with the healthy controls, but not direct evidence of increased BBB permeability. Wang et al. investigated patients with $\mathrm{MCl}$ using DCE-MRI and also observed altered temporal enhancement patterns in the hippocampus (slower decay), indicative of increased BBB permeability ${ }^{26}$. Recently, Montagne et al. used gadobenate dimeglumine as a contrast 
agent and found an elevated BBB impairment in the hippocampus of $\mathrm{MCl}$ patients, which increased with age ${ }^{11}$. Compared with these studies, we found more widespread BBB impairment, analogous to previous findings in (prestages of) vascular dementia ${ }^{9,10,27}$. However, Taheri et al. ${ }^{9,10}$ found that the leakage appeared to be mostly in the WMHs in patients with vascular dementia, which we did not find in the current study. An explanation for this is that the current approach may be more sensitive to the very subtle BBB impairment in patients without obvious cerebrovascular pathology, which would also make it applicable to other diseases which may express diffusely distributed leakage.

The leakage observed in this study can be explained by a breakdown of the BBB tight junctions. It has been shown in rodents that tight junction damage allows gadolinium leakage through the $\mathrm{BBB}^{28}$. The regions with high $\mathrm{BBB}$ leakage were diffusely distributed throughout the brain, showing that BBB tight junctions are globally impaired. This could allow the passage of small and lipophilic molecules that cannot cross a healthy BBB. The loss of tight junctions also changes cell polarity, which influences the expression of transporter complexes and thus indirectly affects active transport across the BBB ${ }^{29}$. Therefore, both passive and active transport mechanisms may be impaired in early AD, possibly disturbing homeostasis ${ }^{1,11}$.

Patients with early AD exhibited a global reduction in local blood plasma volume fraction compared with controls. This points toward widespread cerebrovascular differences between the groups, which is also reflected in the diffusely distributed BBB leakage. The lower local blood plasma volume may be a sign of global hypoperfusion of the brain, which has already been shown using other techniques for measuring cerebral blood flow $^{30}$. Hypoperfusion or ischemia might be an underlying factor of diffuse cerebrovascular endothelial failure leading to leaky blood vessels, but many other pathological mechanisms have also been suggested to contribute in small vessel disease $^{31}$.

We found that cognitive decline was associated with stronger BBB leakage, and both the $\mathrm{MCl}$ and the early $\mathrm{AD}$ patients showed increased BBB leakage. These observations suggest that $B B B$ impairment plays a role in the early pathophysiology of $A D$. A possible mechanism is that loss of tight junctions impairs the filter function of the BBB, leading to a toxic accumulation of substances in the brain. This, combined with the altered active transport systems, might add up to a significant impact on neuronal function that eventually leads to dementia ${ }^{5}$. Inserting this information into the hypothetical model by 
Jack et al. ${ }^{32}$ suggests that BBB impairment would manifest earlier than other structural brain changes, although additional information is needed to compare BBB impairment to amyloid $B$ and tau pathology. The current study does not give information on the interaction between amyloid $B$ and the $B B B$, as amyloid $B$ is actively transported across the $\mathrm{BBB}$, whereas gadolinium leaks passively through the tight junctions ${ }^{4}$. Previous work with PET data has shown that clearance of amyloid $B$ in $A D$ is also impaired ${ }^{33}$. An impaired clearance of amyloid $B$ would mean that the BBB is impaired in different ways, contributing to the pathological cascade leading to AD. BBB leakage may therefore help to provide a biomarker for early diagnosis, or at least a marker indicating vulnerability for the development of dementia. Successful prediction of dementia might eventually lead to optimized treatment, delay or even prevention of the disease.

This study has some potential limitations. First, this is a pilot study with a limited group size. However, the fact that even in this relatively small group highly significant differences can be detected indicates that the effect is substantial and that the methodology is sensitive. Second, the diagnosis of the patients was not confirmed with neuropathology. However, the diagnosis was made using the latest research criteria ${ }^{14,15}$, which are stricter and more reliable than standard clinical criteria.

In conclusion, in this pilot study, MRI was used to show global, diffusely distributed BBB leakage in patients with early $A D$, suggesting that a compromised $B B B$ is part of the early pathology of $A D$, and might be part of a cascade of pathological events eventually leading to cognitive decline.

\section{Acknowledgements}

We thank Frank van Bussel, MSc, Tamar van Veenendaal, MSc, May Wong MSc, and Eleana Zhang, MD, for their assistance with scanning the participants. We would also like to thank Heidi Jacobs for her assistance with obtaining the neuropsychological test scores, Etienne Lemaire for his help in the development of the MRI sequence and we thank Martijn Wolters, MSc, for his implementation of the WMH delineation software. This study was made possible by the "Internationale Stichting Alzheimer Onderzoek" (ISAO, grant 10553). 


\section{References}

$1 \quad$ ladecola C. The Pathobiology of Vascular Dementia. Neuron 2013; 80: 844-866.

2 Srinivasa RN, Rossetti HC, Gupta MK, Rosenberg RN, Weiner MF, Peshock RM et al. Cardiovascular Risk Factors Associated with Smaller Brain Volumes in Regions Identified as Early Predictors of Cognitive Decline. Radiology 2015; 000: 142488.

Benarroch EE. Neurovascular unit dysfunction: A vascular component of Alzheimer disease? Neurology. 2008; 70: 1941.

Zlokovic BV. The Blood-Brain Barrier in Health and Chronic Neurodegenerative Disorders. Neuron 2008; 57: 178-201.

Burgmans S, van de Haar HJ, Verhey FRJ, Backes WH. Amyloid-beta interacts with blood-brain barrier function in dementia: A systematic review. J. Alzheimer's Dis. 2013; 35: 859-873.

Wardlaw JM, Doubal FN, Valdes-Hernandez M, Wang X, Chappell FM, Shuler K et al. Blood-brain barrier permeability and long-term clinical and imaging outcomes in cerebral small vessel disease. Stroke 2013; 44: 525-527.

Erickson MA, Banks WA. Blood-brain barrier dysfunction as a cause and consequence of Alzheimer's disease. J Cereb Blood Flow Metab 2013; 33: 1500-13.

Oostendorp M, Post MJ, Backes WH. Vessel growth and function: depiction with contrast-enhanced MR imaging. Radiology 2009; 251: 317-335.

Taheri S, Gasparovic C, Shah NJ, Rosenberg G a. Quantitative measurement of blood-brain barrier permeability in human using dynamic contrast-enhanced MRI with fast T1 mapping. Magn Reson Med 2011; 65: 1036-1042.

Taheri S, Gasparovic C, Huisa BN, Adair JC, Edmonds E, Prestopnik J et al. Blood-brain barrier permeability abnormalities in vascular cognitive impairment. Stroke 2011; 42: 2158-2163.

Montagne A, Barnes SR, Sweeney MD, Halliday MR, Sagare AP, Zhao Z et al. Blood-Brain Barrier Breakdown in the Aging Human Hippocampus. Neuron 2015; 85: 296-302.

van de Haar HJ, Burgmans S, Hofman PAM, Verhey FRJ, Jansen JFA, Backes WH. Blood-brain barrier impairment in dementia: Current and future in vivo assessments. Neurosci Biobehav Rev 2015; 49C: 71-81.

Jelescu IO, Leppert IR, Narayanan S, Araújo D, Arnold DL, Pike GB. Dual-temporal resolution dynamic contrast-enhanced MRI protocol for blood-brain barrier permeability measurement in enhancing multiple sclerosis lesions. J Magn Reson Imaging 2011; 33: 1291-1300.

Dubois B, Feldman HH, Jacova C, DeKosky ST, Barberger-Gateau P, Cummings J et al. Research criteria for the diagnosis of Alzheimer's disease: revising the NINCDS-ADRDA criteria. Lancet Neurol 2007; 6: 734-746.

McKhann GM, Knopman DS, Chertkow H, Hyman BT, Jack CR, Kawas CH et al. The diagnosis of dementia due to Alzheimer's disease: Recommendations from the National Institute on AgingAlzheimer's Association workgroups on diagnostic guidelines for Alzheimer's disease. Alzheimer's Dement 2011; 7: 263-269.

Morris JC. The Clinical Dementia Rating (CDR): Current version and scoring rules. Neurology 1993; 43: 2412-2412. cognitive state of patients for the clinician. J Psychiatr Res 1975; 12: 189-198. 
Butman J, Gupta S. Automated Computation of the Vascular Input Function for Dynamic ContrastEnhanced MRI of the Brain. In: Proceedings 14th Scientific Meeting, International Society for Magnetic Resonance in Medicine. 2006, p 1535.

Li KL, Zhu XP, Waterton J, Jackson A. Improved 3D quantitative mapping of blood volume and endothelial permeability in brain tumors. J Magn Reson Imaging 2000; 12: 347-357.

Lavini C, Verhoeff JJC. Reproducibility of the gadolinium concentration measurements and of the fitting parameters of the vascular input function in the superior sagittal sinus in a patient population. Magn Reson Imaging 2010; 28: 1420-1430.

Patlak CS, Blasberg RG, Fenstermacher JD. Graphical evaluation of blood-to-brain transfer constants from multiple-time uptake data. J Cereb Blood Flow Metab 1983; 3: 1-7.

Barnes SR, Ng TSC, Montagne A, Law M, Zlokovic BV., Jacobs RE. Optimal acquisition and modeling parameters for accurate assessment of low $\mathrm{K}$ trans blood-brain barrier permeability using dynamic contrast-enhanced MRI. Magn Reson Med 2016; 75: 1967-1977.

Cramer SP, Larsson HBW. Accurate determination of blood-brain barrier permeability using dynamic contrast-enhanced T1-weighted MRI: a simulation and in vivo study on healthy subjects and multiple sclerosis patients. J Cereb Blood Flow Metab 2014; 34: 1655-65.

Benjamini Y, Hochberg Y. Controlling the False Discovery Rate: a Practical and Powerful Approach to Multiple Testing. J R Stat Soc 1995; 57: 289-300.

Starr JM, Farrall AJ, Armitage P, McGurn B, Wardlaw JM. Blood-brain barrier permeability in Alzheimer's disease: a case-control MRI study. Psychiatry Res - Neuroimaging 2009; 171: 232-241.

Wang H, Golob EJ, Su MY. Vascular volume and blood-brain barrier permeability measured by dynamic contrast enhanced MRI in hippocampus and cerebellum of patients with $\mathrm{MCl}$ and normal controls. J Magn Reson Imaging 2006; 24: 695-700.

Hanyu H, Asano T, Tanaka Y, Iwamoto T, Takasaki M, Abe K. Increased blood-brain barrier permeability in white matter lesions of Binswanger's disease evaluated by contrast-enhanced MRI. Dement Geriatr Cogn Disord 2002; 14: 1-6.

Nitta T, Hata M, Gotoh S, Seo Y, Sasaki H, Hashimoto N et al. Size-selective loosening of the bloodbrain barrier in claudin-5-deficient mice. J Cell Biol 2003; 161: 653-660.

Abbott NJ, Rönnbäck L, Hansson E. Astrocyte-endothelial interactions at the blood-brain barrier. Nat Rev Neurosci 2006; 7: 41-53.

Wierenga CE, Hays CC, Zlatar ZZ. Cerebral blood flow measured by arterial spin labeling MRI as a preclinical marker of Alzheimer's disease. J Alzheimers Dis 2014; 42: S411-9.

Wardlaw JM, Smith C, Dichgans M. Mechanisms of sporadic cerebral small vessel disease: Insights from neuroimaging. Lancet Neurol 2013; 12: 483-497.

Jack CR, Knopman DS, Jagust WJ, Shaw LM, Aisen PS, Weiner MW et al. Hypothetical model of dynamic biomarkers of the Alzheimer's pathological cascade. Lancet Neurol 2010; 9: 119-128.

Van Assema DME, Lubberink M, Bauer M, Van Der Flier WM, Schuit RC, Windhorst AD et al. Bloodbrain barrier P-glycoprotein function in Alzheimer's disease. Brain 2012; 135: 181-189 


\section{Appendix}

Table A3.1: Overview of neuropsychological test scores of the patients

\begin{tabular}{|c|c|c|}
\hline Neuropsychological test & Cognitive domain & $\begin{array}{l}\text { Mean Score } \pm \text { standard } \\
\text { deviation }\end{array}$ \\
\hline RAVLT - immediate recall (words) ${ }^{\dagger}$ & Working memory & $24.7 \pm 5.3$ \\
\hline RAVLT - delayed recall (words) ${ }^{\dagger}$ & Episodic memory & $2.0 \pm 2.0$ \\
\hline RAVLT - recognition (words) ${ }^{\dagger}$ & Episodic memory & $20.1 \pm 5.7$ \\
\hline Digit span WAIS-III - forward ${ }^{\dagger}$ & Attention & $7.4 \pm 1.3$ \\
\hline Digit span WAIS-III - backward ${ }^{\dagger}$ & Working memory & $4.4 \pm 1.3$ \\
\hline LDST (correct items after $90 \mathrm{~s})^{\dagger}$ & Processing speed & $29.1 \pm 12.2$ \\
\hline LDST (wrong items after $90 \mathrm{~s})^{\dagger}$ & Processing speed & $0.1 \pm 0.4$ \\
\hline Fluency $\left(\right.$ named animals) ${ }^{\ddagger}$ & Language & $15.1 \pm 5.7$ \\
\hline
\end{tabular}

RAVLT = Rey auditory verbal learning test ${ }^{1}$ (memory), WAIS-III = Wechsler adult intelligence scale third edition ${ }^{2,3}$, LDST $=$ letter digit substitution test ${ }^{4}$

$\dagger$ data missing from 2 subjects

¥ data missing from 1 (other) subject

\section{Image analysis}

T1 and FLAIR weighted images were used for automated, manually adjusted tissue classification of total white matter (WM), total gray matter (GM), deep GM, and cortex (FreeSurfer software package combined with FAST, FMRIB's Automated Segmentation Tool). White matter hyperintensity (WMH) segmentation was performed using a semiautomatic method, and used to determine the WMH volume relative to the intracranial volume and the normal appearing white matter (NAWM) ${ }^{5}$. The Fazekas score and medial temporal lobe atrophy (MTA) rating were determined by an experienced researcher blinded to the subject groups ${ }^{6,7}$.

Motion correction and image registration of the dynamic series were performed using a six degrees of freedom linear transformation with an averaged precontrast image as reference (FLIRT, FMRIB's linear image registration tool, fsl.fmrib.ox.ac.uk/fsl/fslwiki). Further analysis was performed using custom made software in Matlab (version 2012b 
MathWorks, Natick, MA, USA). To account for the nonlinear relationship between signal enhancement and (relatively high) contrast agent concentration of the vascular input function (VIF), the signal conversion was implemented using in vitro data (diluted $\mathrm{MnCl}_{2}$ stock solution with different gadobutrol concentrations (1-40 $\mathrm{mM})$, baseline T1 relaxation time of $1650 \mathrm{~ms}$, comparable to human blood $^{8}$ ). Conversion to contrast agent concentration in the tissue was performed assuming a linear relationship and a contrast agent relaxivity of $3.3 \mathrm{~s}^{-1} \mathrm{mM}^{-19}$.

\section{Noise Suppression}

For the calculation of the leakage rate, the Patlak graphical approach ${ }^{10}$ is used, which is based on linear fits of scatterplots. The slope of this fit is the blood-brain barrier (BBB) leakage rate $\mathrm{K}_{\mathrm{i}}$ (assuming a tissue density of $1 \mathrm{~g} / \mathrm{ml}$ ), and the intercept is the local blood plasma volume $v_{p}$. However, the scatterplots were rather noisy due to the very low permeability of brain tissue, thus yielding not only positive, but also negative slope values. To further increase the sensitivity of this leakage detection, a histogram approach was used. The histograms were normalized and noise was estimated by assuming that negative slope values can only be attributed to noise, and that a similar distribution of noise is also present in positive slope values. The data were then corrected by subtracting the estimated noise from the measured histogram. The remaining cumulative sum of the bins was defined as the BBB leakage volume fraction VL.

\section{Dynamic susceptibility contrast (DSC)-MRI}

DSC-MRI was performed to determine the local cerebral blood flow and blood volume.

Acquisition. The DSC-MRI was performed by a 3D gradient echo (PRESTO) sequence ${ }^{11}$ with a TR/TE of $20 / 30 \mathrm{~ms}$, flip angle of $8^{\circ}$, voxel size of $1.9 \times 1.9 \times 3.5 \mathrm{~mm}^{3}$, matrix size of $128 \times 128 \times 30$, Dynamic Scan Interval (DSI) of $1.56 \mathrm{~s}$ with 75 volumes, resulting in a total scan time of $117 \mathrm{~s}$. Contrast agent (gadobutrol, $0.1 \mathrm{~mm} / \mathrm{kg}$, injected using a power injector with a flow of $3 \mathrm{ml} / \mathrm{s}$, followed by a $20 \mathrm{ml}$ saline flush) was injected at the $11^{\text {th }}$ dynamic scan. The DSC-MRI sequence was performed after the DCE-MRI sequence.

Analysis. The motion correction was performed using the mean of the precontrast images as reference. The raw signal-time series were converted to concentration using

$$
C(t)=-k \cdot\left(\frac{1}{\mathrm{TE}}\right) \cdot \ln \left(\frac{S(t)}{S_{0}}\right)
$$


Where $C(t)$ is the contrast agent concentration over time, $k$ is the proportionality constant for MRI in brain tissue, $S(t)$ is the signal at time $t$ and $S_{0}$ is the baseline signal before contrast agent arrival. The proportionality constant $k$ was set to 1 in this study, under the assumption that the transverse relaxivity $\left(r_{2}{ }^{*}\right)$ of tissue and blood is equal, and a change in contrast agent concentration gives a linear increase on the transverse relaxation rate $\left(R_{2}{ }^{*}\right)$. An individual vascular input function (VIF) was chosen in or near the anterior cerebral artery. Cerebral blood volume (CBV) was calculated using

$$
\mathrm{CBV}=\frac{\left(1-H_{l}\right) \cdot \int_{0}^{a} C(t) d t}{\rho \cdot\left(1-H_{s}\right) \cdot \int_{0}^{a} \operatorname{VIF}(t) d t}
$$

Where $H_{l}$ and $H_{s}$ are the hematocrit values in the large and small blood vessels, respectively, $\rho$ is the brain mass density, and $a$ is the time point at which the contrast agent concentration reaches baseline again after the initial injection peak. The total correction factor $\left(1-H_{l}\right) / \rho\left(1-H_{s}\right)$ was set as $0.705 \mathrm{~cm}^{3} / \mathrm{g}^{12}$. Voxels with CBV values above $10 \%$ were considered to represent large blood vessels and were discarded for all further analyses. The CBF was calculated solving

$$
C(t)=\mathrm{CBF} \cdot R(t) \otimes \mathrm{VIF}
$$

Where $R(t)$ is the tissue residue function and $\otimes$ denotes the convolution operator ${ }^{13}$. The deconvolution was performed using a block-circulant singular value decomposition method with a cutoff value of $10 \%$, meaning that singular values below $10 \%$ of the maximal singular value were set to zero ${ }^{13,14}$.

Statistics. Independent two-sided Student's $t$-tests were used to test group differences in CBF and CBV in two steps; first the differences between the groups in the total WM and GM were tested, and in the second step, the NAWM, deep GM, cortex and WMHs. To test whether $\mathrm{CBF}$ and $\mathrm{BBB}$ leakage were correlated, the Pearson correlation coefficient between $C B F$ and $B B B$ leakage rate $K_{i}$ and $V_{L}$ was calculated in every region over all subjects, using the same two-step approach.

Results. An overview of the results is given in table A3.2. In short, there were no significant differences in CBF or CBV between the patients and controls in any of the investigated regions. No significant correlations were found between the CBF and the BBB leakage measures. 
Conclusion. Both the CBF and the CBV values are comparable to those reported in the literature ${ }^{15,16}$. Using $D S C-M R I$, patients with early $A D$ and healthy controls had comparable CBF and CBV values and CBF did not correlate with BBB leakage.

Table A3.2: Regional comparison of DSC-MRI measures and correlation with bloodbrain barrier leakage

\begin{tabular}{|c|c|c|c|c|c|c|c|c|c|c|}
\hline \multirow[b]{2}{*}{ ROI } & \multicolumn{3}{|c|}{ CBF $(\mathrm{ml} / 100 \mathrm{~g} / \mathrm{min})$} & \multicolumn{3}{|l|}{ CBV (\%) } & \multicolumn{2}{|c|}{$\begin{array}{l}\text { Correlation CBF } \\
\text { and } K_{i}\end{array}$} & \multicolumn{2}{|c|}{$\begin{array}{l}\text { Correlation CBF } \\
\text { and } v_{L}\end{array}$} \\
\hline & Patients & Controls & $\mathrm{p}$ & Patients & Controls & $\mathrm{p}$ & Pearson's r & $\mathrm{p}$ & Pearson's r & $\mathrm{p}$ \\
\hline WM & $\begin{array}{l}53.0 \pm \\
21.6\end{array}$ & $\begin{array}{l}54.0 \pm \\
29.7\end{array}$ & 0.9 & $\begin{array}{l}3.4 \pm \\
0.8\end{array}$ & $3.6 \pm 1.2$ & 0.7 & -0.14 & 0.5 & -0.10 & 0.6 \\
\hline GM & $\begin{array}{l}84.7 \pm \\
35.4 \\
\end{array}$ & $\begin{array}{l}81.7 \pm \\
40.1\end{array}$ & 0.8 & $\begin{array}{l}5.2 \pm \\
1.1 \\
\end{array}$ & $5.2 \pm 1.5$ & 1.0 & -0.11 & 0.6 & -0.22 & 0.3 \\
\hline NAWM & $\begin{array}{l}53.4 \pm \\
21.5\end{array}$ & $\begin{array}{l}54.5 \pm \\
29.6\end{array}$ & 0.9 & $\begin{array}{l}3.4 \pm \\
0.8\end{array}$ & $3.6 \pm 1.2$ & 0.7 & -0.14 & 0.5 & -0.12 & 0.5 \\
\hline $\begin{array}{l}\text { Deep } \\
\text { GM }\end{array}$ & $\begin{array}{l}84.6 \pm \\
36.6\end{array}$ & $\begin{array}{l}78.3 \pm \\
38.0\end{array}$ & 0.7 & $\begin{array}{l}5.1 \pm \\
1.2\end{array}$ & $5.0 \pm 1.5$ & 0.8 & -0.15 & 0.4 & 0.04 & 0.8 \\
\hline Cortex & $\begin{array}{l}84.8 \pm \\
35.3\end{array}$ & $\begin{array}{l}82.2 \pm \\
40.3\end{array}$ & 0.9 & $\begin{array}{l}5.2 \pm \\
1.1\end{array}$ & $5.2 \pm 1.5$ & 1.0 & -0.11 & 0.6 & -0.19 & 0.3 \\
\hline WMHs & $\begin{array}{l}42.0 \pm \\
16.6\end{array}$ & $\begin{array}{l}43.2 \pm \\
21.6\end{array}$ & 0.9 & $\begin{array}{l}3.0 \pm \\
0.7\end{array}$ & $3.1 \pm 1.0$ & 0.9 & -0.23 & 0.2 & -0.19 & 0.3 \\
\hline
\end{tabular}

All data (other than $\mathrm{p}$ values) are mean \pm one standard deviation. $\mathrm{ROI}=$ region of interest, $\mathrm{CBF}=$ cerebral blood flow, $\mathrm{CBV}=$ cerebral blood volume, $\mathrm{WM}=$ white matter, $\mathrm{GM}=$ gray matter, NAWM = normal appearing white matter, WMHs = white matter hyperintensities. 


\section{References}

1

2

3

4

5

6

7

Rey A. L'examen clinique en psychologie. Presse Universitaires de France: Paris, 1964.

Wechsler D. WAIS-III/WMS-III Technical Manual Update, 3rd ed. Psychological Corporation: San Antonio, 1997.

Borkowski JG, Benton AL, Spreen O. Word fluency and brain damage. Neuropsychologia 1967; 5: 135-140.

van der Elst W, van Boxtel MPJ, van Breukelen GJP, Jolles J. The Letter Digit Substitution Test: normative data for 1,858 healthy participants aged 24-81 from the Maastricht Aging Study (MAAS): influence of age, education, and sex. J Clin Exp Neuropsychol 2006; 28: 998-1009.

de Boer R, Vrooman HA., van der Lijn F, Vernooij MW, Ikram MA, van der Lugt A et al. White matter lesion extension to automatic brain tissue segmentation on MRI. Neuroimage 2009; 45: 1151-1161.

Fazekas F, Kleinert R, Offenbacher H, Schmidt R, Kleinert G, Payer F et al. Pathologic correlates of incidental MRI white matter signal hyperintensities. Neurology 1993; 43: 1683-1689.

Scheltens P, Leys D, Barkhof F, Huglo D, Weinstein HC, Vermersch P et al. Atrophy of medial temporal lobes on MRI in 'probable' Alzheimer's disease and normal ageing: diagnostic value and neuropsychological correlates. J Neurol Neurosurg Psychiatry 1992; 55: 967-972.

Lu H, Clingman C, Golay X, Van Zijl PCM. Determining the longitudinal relaxation time (T1) of blood at 3.0 tesla. Magn Reson Med 2004; 52: 679-682.

Pintaske J, Martirosian P, Graf H, Erb G, Lodemann K-P, Claussen CD et al. Relaxivity of Gadopentetate Dimeglumine (Magnevist), Gadobutrol (Gadovist), and Gadobenate Dimeglumine (MultiHance) in human blood plasma at 0.2, 1.5, and 3 Tesla. Invest Radiol 2006; 41: 213-221.

10 Patlak CS, Blasberg RG, Fenstermacher JD. Graphical evaluation of blood-to-brain transfer constants from multiple-time uptake data. J Cereb Blood Flow Metab 1983; 3: 1-7.

1 Liu G, Sobering G, Duyn J, Moonen CTW. A. functional MRI technique combining principles of echoshifting with a train of observations (PRESTO). Magn Reson Med 1993; 30: 764-768.

2 Knutsson L, Lindgren E, Ahlgren A, van Osch MJP, Markenroth Bloch K, Surova Y et al. Dynamic susceptibility contrast MRI with a prebolus contrast agent administration design for improved absolute quantification of perfusion. Magn Reson Med 2013; 1006: 996-1006.

Willats L, Calamante F. The 39 steps: Evading error and deciphering the secrets for accurate dynamic susceptibility contrast MRI. NMR Biomed 2013; 26: 913-931.

Wu O, Østergaard L, Weisskoff RM, Benner T, Rosen BR, Sorensen AG. Tracer arrival timinginsensitive technique for estimating flow in MR perfusion-weighted imaging using singular value decomposition with a block-circulant deconvolution matrix. Magn Reson Med 2003; 50: 164-174.

Hauser T, Schönknecht P, Thomann PA, Gerigk L, Schröder J, Henze R et al. Regional Cerebral Perfusion Alterations in Patients with Mild Cognitive Impairment and Alzheimer Disease Using Dynamic Susceptibility Contrast MRI. Acad Radiol 2013; 20: 705-711.

Engvall C, Ryding E, Wirestam R, Holtås S, Ljunggren K, Ohlsson T et al. Human Cerebral Blood Volume (CBV) measured by dynamic susceptibility contrast MRI and 99mTc-RBC SPECT. J Neurosurg Anesth 2008; 20: 41-44. 



\section{Chapter 4}

\section{Subtle blood-brain barrier}

\section{leakage rate and spatial extent:}

considerations for dynamic contrast-enhanced MRI

Harm J van de Haar, Jacobus FA Jansen, Cécile RLPN Jeukens, Matthias JP van Osch, Mark A van Buchem, Majon Muller, Paul AM Hofman, Frans RJ Verhey, Saartje Burgmans, Walter $\mathrm{H}$ Backes 


\section{Abstract}

Dynamic contrast-enhanced (DCE) MRI can be used to measure blood-brain barrier (BBB) leakage. However, in neurodegenerative disorders such as small vessel disease and dementia, the leakage can be very subtle and the measured signal can be noisy, which requires an optimized DCE-MRI measurement and study design. To help improve future studies, a new measure indicative of the spatial extent of leakage is introduced and the effects of scan time and sample size are explored. Dual-time resolution DCE-MRI was performed in 16 patients with early Alzheimer's disease (AD) and 17 healthy controls. The leakage rate $\left(K_{i}\right)$ and volume fraction of detectable leaking tissue $\left(V_{L}\right)$ were calculated in cortical gray matter and white matter using noise-corrected histogram analysis of leakage maps. Computer simulations utilizing realistic $K_{i}$ histograms, mimicking the strong effect of noise and variation in $K_{i}$ values, were performed to understand the influence of scan time on the estimated leakage. The mean $K_{i}$ was very low (in the order of $10^{-4} \mathrm{~min}^{-1}$ ) and highly influenced by noise, causing the $\mathrm{K}_{\mathrm{i}}$ to be increasingly overestimated at shorter scan times. In the white matter, the $K_{i}$ was not different between patients with early $A D$ and controls, but was higher in the cortex for patients, reaching significance after 14.5 minutes of scan time. To detect group differences, VL proved more suitable, showing significantly higher values for patients compared with controls in the cortex after 8 minutes of scan time, and in white matter, VL was significantly higher in patients after 15.5 minutes. Several ways to improve the sensitivity of a DCE-MRI experiment to subtle BBB leakage were presented. We have shown how increasing the scan time affects the $\mathrm{K}_{\mathrm{i}}$ estimation, and have provided $\mathrm{V}_{\mathrm{L}}$ as an attractive and potentially more time-efficient alternative to detect group differences in subtle and widespread blood-brain barrier leakage compared with leakage rate $\mathrm{K}_{\mathrm{i}}$. Recommendations on group size and scan time are made based on statistical power calculations to aid future research. 


\section{Introduction}

Impairment of the blood-brain barrier (BBB) is thought to be a key mechanism in the pathophysiology of several brain diseases such as small vessel disease, Alzheimer's disease $(A D)$ and multiple sclerosis ${ }^{1-4}$. A suitable method to investigate this impairment is dynamic contrast-enhanced (DCE) MRI, which is a non-invasive imaging technique that follows the arrival and distribution of a contrast agent over time. The most common contrast agents in MRI are gadolinium-based compounds, which almost completely remain intravascular in a healthy brain due to the $\mathrm{BBB}^{5,6}$. The $\mathrm{BBB}$ is a collection of anatomical elements in the wall of brain capillaries. It protects the neuronal tissue from neurotoxic compounds, while allowing essential molecules such as oxygen or nutrients to pass. When damaged, certain substances can pass the BBB more easily, which may be harmful to the brain parenchyma. In case of pathology, such as (high-grade) tumors or infarctions, microvessels become hyperpermeable and the contrast agent may extravasate more easily and accumulate in the brain parenchyma. This leakage can be detected, and the difference between intravascular and extravascular contrast agent concentration can be quantified using DCE-MRI ${ }^{7}$. However, compared to high-grade tumors and infarcted brain tissue, the leakage in neurodegenerative disorders such as Alzheimer's disease is found to be very subtle, making it much more difficult to discriminate between intra- and extravascular contrast agent ${ }^{8-10}$. Furthermore, the spatial extent and distribution of the leakage is also of interest. Contrary to tumors, the leakage in a neurodegenerative disease may not be localized at an obvious site, making detection of the leakage within a large region more important. A priori, it remains unknown what better characterizes the BBB damage in a neurodegenerative disease; the magnitude of the leakage rate or the spatial extent of the leakage. Furthermore, investigating this also requires a more sensitive technique compared with tumors, as the leakage is orders of magnitude lower in the brain tissue and difficult to distinguish from noise.

To measure such subtle diffuse leakage, the DCE-MRI methodology has to be further modified to detect low concentrations of contrast agent and slower signal changes over time. Previous studies have looked at the most optimal pharmacokinetic model and the ability to distinguish low leakage from zero ${ }^{6,11}$. However, improving the DCE-MRI experiment to voxelwise mapping of the leakage remains difficult. A straightforward way to improve the sensitivity for subtle leakage is to scan longer, which increases the amount of available data from which leakage can be estimated, and also allows the 
contrast agent more time to extravasate and accumulate in the tissue. In the majority of studies which apply DCE-MRI in neurodegenerative diseases, a protocol of roughly 20-30 minutes is used ${ }^{9,10,12-14}$, although some recent studies use shorter protocols of 16 minutes ${ }^{6,15}$. In a group study, the sensitivity to subtle leakage can also be improved by increasing the group sizes. Additionally, the analysis method can be further improved, reducing the impact of noise on the leakage quantification.

The aim of this study is to explore several ways to improve the ability to detect widespread subtle BBB leakage in neurodegenerative diseases. First, we explore the effect of changing the acquisition time on the measured leakage. Second, to determine the spatial extent of the lowest possible leakage rates, we propose a noise removal method and the alternative leakage measure 'volume fraction of detectable leaking brain tissue voxels' $(\mathrm{V} L)$, which may help to distinguish very low leakage from noise. We further investigated this using data from in vivo DCE-MRI measurements in patients with early $A D$ and healthy age-matched controls. To obtain more insight into the relation between scan time and measurement of subtle BBB leakage, computer model simulations were implemented, using representative leakage rate $\left(K_{i}\right)$ histograms that mimic the effect of voxelwise mapping of the leakage measures with in vivo noise and realistic variations in measured $\mathrm{K}_{\mathrm{i}}$ values. In addition, we investigated the scan time and group size necessary to detect significant group differences, which may assist to motivate the best study design for future DCE-MRI studies. Data from both the white matter and the cortical gray matter are reported, as different requirements are expected for tissues with different degrees of vascularization.

\section{Materials and Methods}

\section{Participants}

Sixteen patients with early $A D$ (mean age 73.6 s.d. 7.9 years, 7 women), defined as being diagnosed with either mild dementia of the $A D$ type or mild cognitive impairment due to $A D$, and 17 healthy age-matched controls (mean age 75.8 s.d. 6.2 years, 6 women) were included. All subjects underwent MRI at the Maastricht University Medical Center or Leiden University Medical Center. Subjects were excluded in case of contraindications for MRI, renal dysfunction, major structural brain abnormalities, alcohol/drug abuse, or other major vascular, psychiatric or neurological disorders. This study was approved by both the Medical Ethical Committee AZM/UM and the Committee for Medical Ethics 
LUMC. Informed consent was obtained from all patients after they received verbal and written descriptions of the study.

\section{Image acquisition}

To detect BBB leakage, a dual time resolution dynamic contrast-enhanced imaging protocol was implemented for 3 T MRI (Philips Achieva, Best, the Netherlands). This protocol consisted of two nested pulse sequences, a slow and a fast sequence. This approach is comparable to the imaging protocol employed by Jelescu et al., which was found to be more precise and less biased at estimating BBB impairment in multiple sclerosis, compared with a conventional single time resolution scan, and was also found to be relatively insensitive to perfusion differences ${ }^{16}$. The slow sequence started immediately after the fast sequence, for a total time of 25 minutes, including 3 precontrast (slow sequence) volumes which are needed for further analysis. All reported scan times include these 3 pre-contrast scans, the fast sequence and the postcontrast slow sequence volumes. In the dual time resolution scan, the fast sequence allowed for a higher temporal resolution during initial arrival and recirculation of the contrast agent, while the slow sequence provided a better SNR, but lower temporal resolution during contrast agent distribution and washout. Both sequences are based on a previously described sequence, for which the relation between signal and longitudinal relaxation rate is known and can be used for T1 mapping ${ }^{5}$. The fast sequence was a saturation recovery $3 \mathrm{D}$ gradient recalled sequence (TR/TE $5.2 / 2.5 \mathrm{~ms}$, flip angle $30^{\circ}, 25.6 \times 20 \times 5$ $\mathrm{cm}^{3} \mathrm{FOV}$ ), 256x200x10 matrix, dynamic scan interval (DSI) $3.2 \mathrm{~s}$ ) with a saturation prepulse given at a delay time (TD) of $120 \mathrm{~ms}$, used during bolus injection (gadobutrol, 0.1 $\mathrm{mmol} / \mathrm{kg}$ ) for 1.5 minutes. The slow sequence was also a saturation recovery gradient

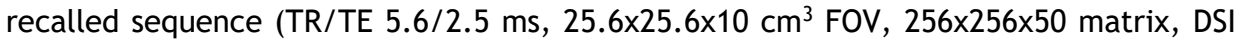
$30.5 \mathrm{~s})$ with the same pre-pulse. The two sequences were combined by upsampling the volumes of the fast sequence using linear interpolation to match the slow sequence FOV and voxel size. Because the sequences were combined on the MRI console, the time indices of each slow and fast volume relative to the start of the DCE-MRI sequence were present in the exported data. T1 weighted structural images were used for automated and manually adjusted tissue classification into white matter (WM) and gray matter (GM) using the FreeSurfer software package (version 5.1.0) ${ }^{17}$. A fluid attenuated inversion recovery (FLAIR) scan was used to segment white matter hyperintensities, which were excluded from the WM mask to create the normal appearing white matter (NAWM) mask $^{18}$. 


\section{Leakage analysis}

The sequential images underwent correction for head displacement using a mutual information algorithm with an averaged pre-contrast image as reference (FLIRT, the linear image registration tool of the Oxford Centre for functional MRI of the brain (FMRIB), http://fsl.fmrib.ox.ac.uk/fsl/fslwiki/FLIRT). An individual vascular input function (VIF) was extracted per participant from the superior sagittal sinus, also used by others ${ }^{16,19-21}$. Further analysis was performed using in-house developed software implemented in Matlab (version 2012b MathWorks, Natick, MA, USA). Translation of the signal enhancement to contrast agent concentration was performed in two ways. For the VIF, the signal enhancement was converted to the contrast agent concentration using in vitro concentration calibrations. These calibrations were performed using a diluted $\mathrm{MnCl}_{2}$ stock solution with a baseline T1 of $1650 \mathrm{~ms}$, which is comparable to human blood. The signal change caused by different gadobutrol concentrations (1-40 mM) in the stock solution was measured for both the fast and the slow sequence. For the brain tissue, the expectedly low contrast agent concentration was calculated assuming a linear relationship between signal change and contrast agent concentration and using a contrast agent relaxivity of $3.3 \mathrm{~s}^{-1} \mathrm{mM}^{-1} 22$. This approximation is valid for the low concentration found in brain tissue in our experiments ${ }^{23}$. Using phantoms with two different gadobutrol concentrations (1 and $40 \mathrm{mM}$ ), we measured the drift over the course of the DCE-MRI sequence. The baseline T1 values were based on T10 measurements using a sequence comparable to the slow sequence but with different TD values $(120-4000 \mathrm{~ms})^{5}$. The mean T10 values for the tissue of the subjects was calculated, and the individual segmentations of the T1 structural scans were used to assign voxelwise T10 values to the WM and GM. These T10 maps were smoothed with a $2 \times 2 \times 2$ kernel to account for partial volume effects of the tissue. Next, a twocompartment pharmacokinetic model was applied voxel-wise, using the Patlak graphical approach $^{24}$. The Patlak plot provided the BBB leakage rate $\left(\mathrm{K}_{\mathrm{i}}\right.$, in $\left.\mathrm{min}^{-1}\right)$ from the slope, and the fractional blood plasma volume $\left(v_{p}\right)$ from the intercept (assuming a hematocrit level of $45 \%$ ). The $v_{p}$ was not further investigated in this study as it was mainly dependent on the data from the fast DCE-MRI sequence, and appeared relatively insensitive to the total scan time including the slow sequence ${ }^{25}$. The median $K_{i}$ of all voxels within the NAWM and GM per subject was calculated, which is for simplicity referred to as leakage rate $K_{i}$ for the remainder of this article. 


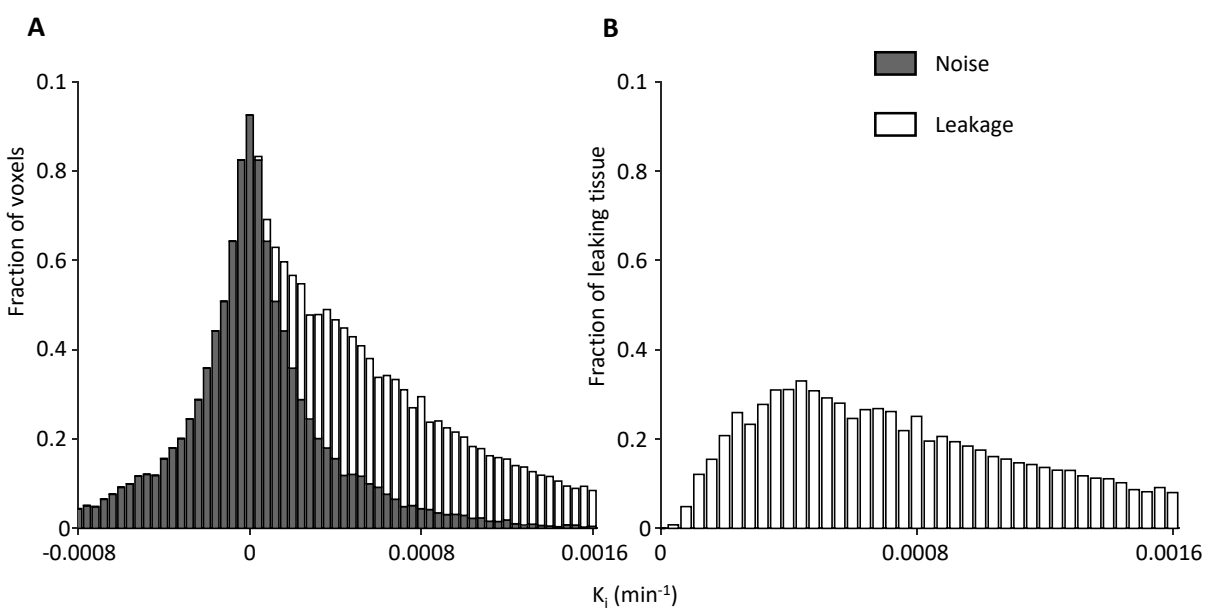

Figure 4.1: An overview of the histogram method in the gray matter of a control subject $(A)$ and the result of noise correction (B). By assuming that all bins with negative $K_{i}$ values are due to noise, the noise in the positive bins can be estimated by mirroring the negative bins to the positive side. The remaining bins are then assumed to represent the voxels that exhibit leakage that can be differentiated from noise (in B).

\section{Noise correction and fractional volume of leaking tissue calculation}

Due to noise on the concentration time curves, the slope of the Patlak plot was occasionally negative, which directly translates to a $\mathrm{K}_{\mathrm{i}}$ value smaller than zero, which is physiologically meaningless. Therefore, we assumed that all negative $K_{i}$ values were erroneous and caused by noise on the concentration time curves. Since noise on top of the signal in a non- (or negligibly-) leaking voxel would equally likely result in a negative as well as a positive $K_{i}$, we also assumed that the noise would give rise to an equal distribution on the positive side. Further investigation of the observed $K_{i}$ histograms showed that the distribution was skewed towards the upper tail containing high $\mathrm{K}_{\mathrm{i}}$ values, which we consider to be likely due to actual leakage instead of noise (Figure 4.1A). By mirroring the negative bins to the positive side, the total noise was estimated and subsequently removed from the histograms, therefore classifying each $K_{i}$ value as either due to noise or leakage. The bins remaining after noise correction were considered to represent the distribution of voxels that exhibit detectable leakage (Figure 4.1B). Note that the bins remaining after noise removal do not represent the 
complete distribution of the leaking brain tissue, but only the detectable part. The cumulative sum of the resulting bins was defined to represent the detectable fractional volume of leaking tissue: VL. Therefore, $V L$ is a measure of the fraction of voxels that exhibit a detectable leakage in an ROI. A higher $V_{L}$ would indicate an increase of voxels showing leakage, but could also be indicative for an improved detection method. A higher $K_{i}$ would indicate a stronger overall leakage. An example to illustrate the difference between $K_{i}$ and $V_{L}$ is displayed in Figure 4.2, which shows two leakage distributions with roughly equal average $K_{i}$ values but a very different $V_{L}$. The behavior of $\mathrm{V} L$ and $K_{i}$ when different input distributions are measured is displayed in Figure 4.3. This figure shows how $V L$ is able to differentiate non-leaking voxels from voxels exhibiting leakage, and how altering the leakage rate and proportion of leaking voxels affects the mean $K_{i}$ and $\mathrm{VL}$.

The aim of this study is to provide information on how to improve the experimental setup of future studies. To facilitate easy comparisons between this study and previous DCE-MRI studies, all $K_{i}$ values reported in this study were calculated without usage of the noise suppression method. The noise suppression method is only utilized to calculate the $\mathrm{V} L$, and any $\mathrm{K}_{\mathrm{i}}$ mentioned in this study is based on the raw (and noisy) values. 
A

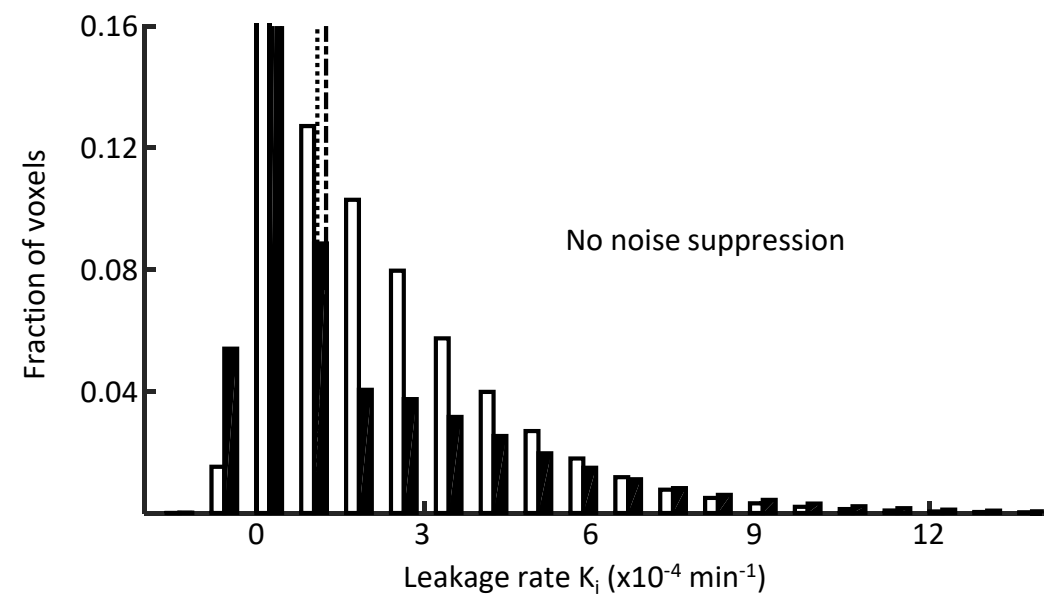

B

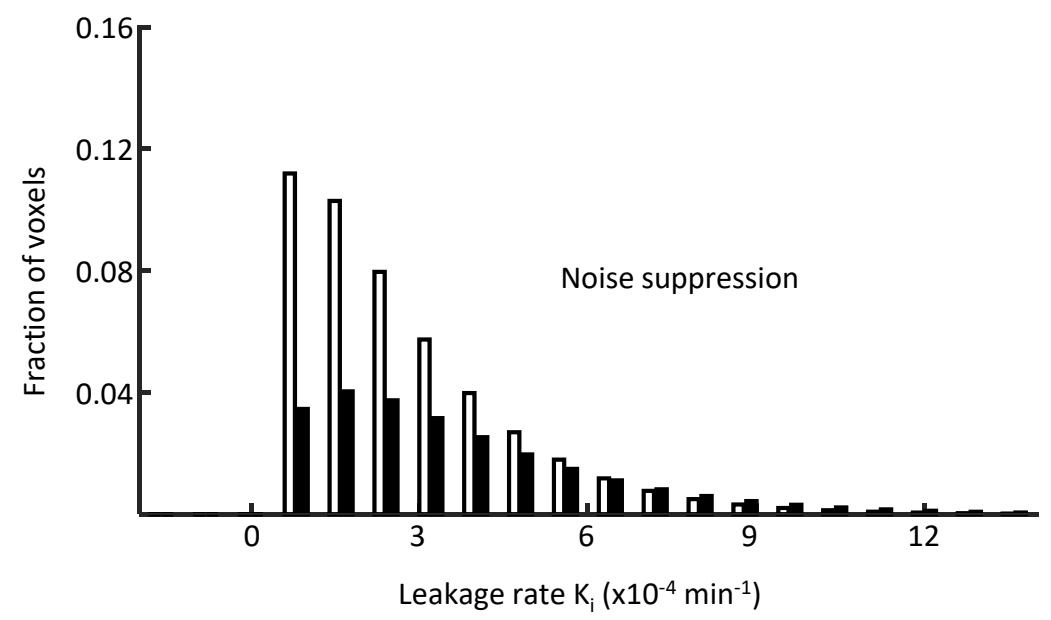

Figure 4.2: An illustration of the effect of the noise suppression on two different histograms. The leakage distributions of $A$, which still exhibit noise and negative leakage rate values, have almost the same mean $\mathrm{K}_{\mathrm{i}}$ (black distribution/dotted line: $1.09 \times 10^{-4} \mathrm{~min}^{-1}$, white distribution/dash-dotted line: $1.24 \times 10^{-4} \mathrm{~min}^{-1}$ ) with a percentage difference of $14 \%$, with the most notable differences in the section of the lowest $K_{i}$ values. The white distribution has more low $K_{i}$ values, close to the zero level, than the black distribution. After noise suppression, the difference between the distributions becomes more clear, showing relatively more voxels exhibiting leakage in the lowest leakage rate range for the white distribution. By calculating $\mathrm{V}_{\mathrm{L}}$, the sum of the bins, this difference becomes quantitatively apparent, revealing a much higher percentage difference of $105 \%$ between the two distributions (black: 0.19 , white: 0.40 ). 
A

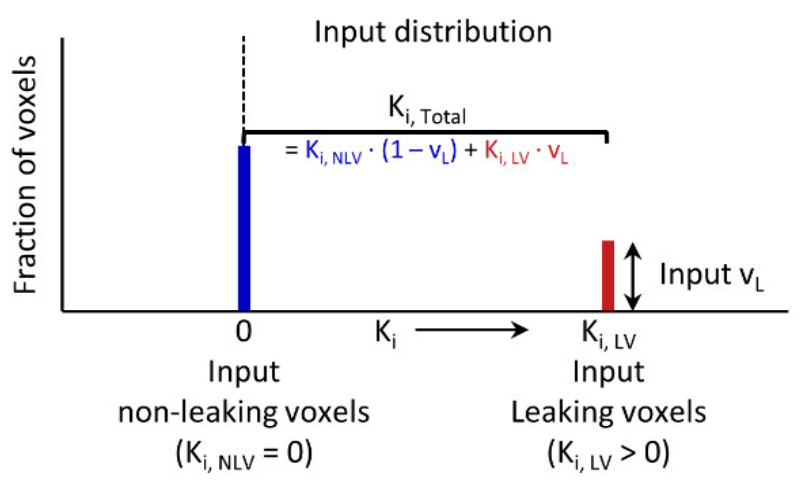

B
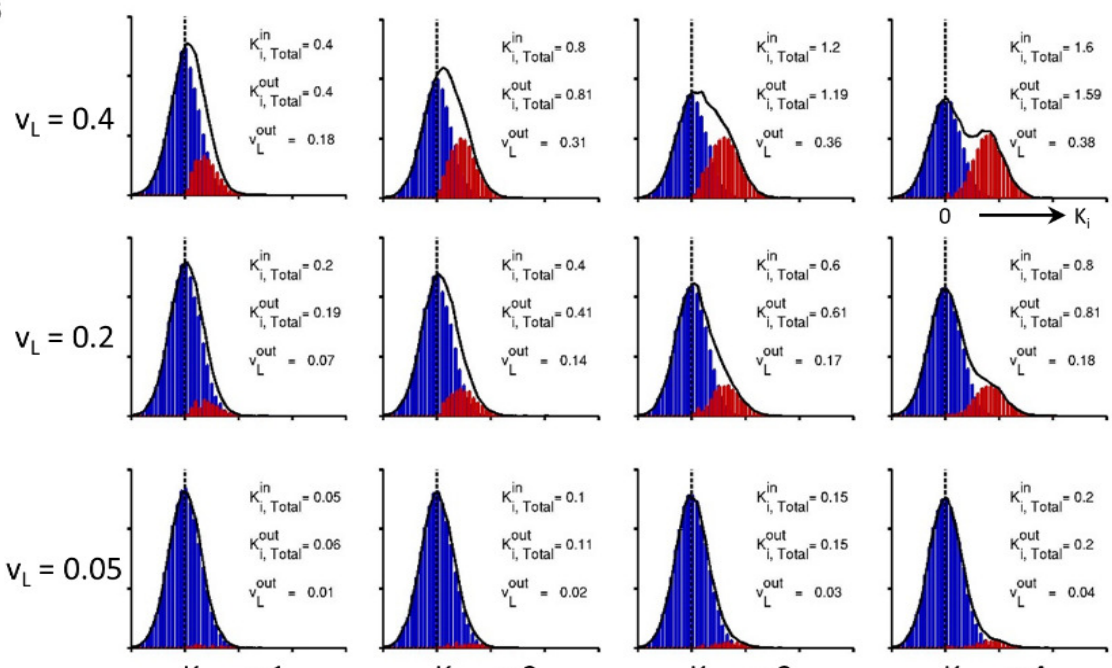

$\mathrm{K}_{\mathrm{i}, \mathrm{LV}}=3$

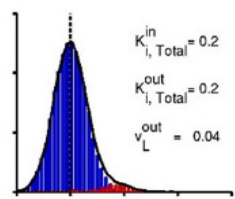

$\mathrm{K}_{\mathrm{i}, \mathrm{LV}}=4$

Figure 4.3: Simulations illustrating the behaviour of $K_{i}$ and $V L$ when altering the ratio and leakage value of leaking versus non-leaking voxels. The basic input distribution is shown in A. In short, the voxels were divided into a non-leaking and leaking portion, the latter of which is the input $\mathrm{V}_{\mathrm{L}}$. The relative proportion of the leaking voxels (input $\mathrm{V}_{\mathrm{L}}$ ) and leakage rate $\left(\mathrm{K}_{\mathrm{i}, \mathrm{LV}}\right)$ was varied to create the different input distributions. After adding noise to the concentration curves (for details, see the Methods section), the $K_{i}$ values were recalculated and the resulting histogram was analyzed in the way described in the manuscript. The $\mathrm{K}_{\mathrm{i}}$, Total displayed in the graphs combines the $\mathrm{K}_{\mathrm{i}}$ of the non-leaking and leaking voxels into the total distribution (denoted by the black curve), which is equal to $\mathrm{K}_{\mathrm{i}}$, Total $=$ $\mathrm{VL} \cdot \mathrm{K}_{\mathrm{i}, \mathrm{LV}}$ (because $\mathrm{K}_{\mathrm{i}, \mathrm{NLV}}=0$ ). The figure shows that the measured (total) $\mathrm{K}_{\mathrm{i}}$ is not equal to the (input) $\mathrm{K}_{\mathrm{i}, \mathrm{LV}}$ of the leaking voxels. It also demonstrates that the relative volume of leaking voxels $\mathrm{V} L$ is more accurately measured when either the relative amount of leaking voxels or the leakage rate of the leaking voxels increases. For these simulations, a scan time of 15 minutes and a signal-to-noise ratio of 1.68 was used. All $\mathrm{K}_{\mathrm{i}}$ values are $\times 10^{-4} \mathrm{~min}^{-1}$. 


\section{Statistical analysis}

A two-sided Student's $t$-test was used to determine significant differences in (median) $\mathrm{K}_{\mathrm{i}}$ and $\mathrm{VL}$ between patients and controls in the NAWM and cortex. Statistical significance was inferred when $p<0.05$.

To determine how shortening of the scan time affects $K_{i}$ and $V L$, the statistical tests were repeated for shorter scan times by discarding the data beyond that time. For example, for a scan time of 10 minutes, the Patlak plot of each relevant voxel was constructed with data obtained from $\mathrm{t}=0$ to 10 minutes.

\section{Sample size estimation}

The imaging data also allowed for a post-hoc power analysis, which was used to estimate the impact of group size and scan time on the statistical power. We used the measured effect and standard deviation as a function of scan time in both the NAWM and cortex over the subjects to calculate the $80 \%$ power levels for different effect sizes and group sizes using ${ }^{26} \mathrm{~N} \approx 2 \sigma^{2}\left(\mathrm{Z}_{\alpha}+\mathrm{Z}_{1-\beta}\right)^{2} / \Delta^{2}$. Here, $\mathrm{N}$ is the group size, $\sigma$ is the pooled standard deviation over the subjects, $\Delta$ is the (absolute) mean difference in $K_{i}$ or VL between the groups (i.e. effect size), $a$ is the probability of a type I error (set at $5 \%$ ), and $B$ is the probability of a type II error (the power, 1-B, set at $80 \%$ ). Smaller effect sizes were expressed as a fraction of the measured effect size, i.e. an effect size of $0.75 \Delta \mathrm{V}$ indicates that the difference in $\mathrm{V} L$ between the patients and controls was reduced by $25 \%$. The difference between the mean of the patients and controls, $\Delta$, was based on the imaging data and thus depended on the scan time. These calculations served to indicate the relation between group size and scan time, and the effect on the power for various $\Delta$ values.

\section{Simulations}

To further investigate the effect on the measured $K_{i}$ and $V L$ when the scan time is shortened, computer model simulations of concentration time-curves were performed using Matlab. A VIF was calculated from the mean blood curve data of the controls by fitting a linear curve to the arrival phase, and a biexponential curve to the washout phase of the contrast agent concentration time-course ${ }^{27}$. SNR's were calculated from the concentration curves in the white matter of the control subjects (SNR = mean / standard deviation from $\mathrm{t}=20$ to 25 minutes) and used to introduce random noise to the simulated tissue concentration curves. To simulate realistic $K_{i}$ values of the region of 
interest (ROI), a Pareto distribution was used to provide input $K_{i}$ values, as previously described by Taheri et al. ${ }^{13}$. Although the true BBB leakage distribution is unknown due to the absence of a golden standard, we consider the Pareto distribution a reasonable model as it comprises a long tail combined with a relatively high fraction of voxels with subtle $K_{i}$ values. It is given by the formula

$$
f(x)=\frac{a b^{a}}{x^{a+1}} \quad \text { for } x \geq b
$$

where $f(x)$ gives the relative incidence of a $K_{i}$ value $(x)$ and $a$ and $b$ define the shape and scale of the distribution, respectively. We varied $a$ and $b$ to examine different leakage histograms (Figure 4.4). Note that only positive $K_{i}$ values were included for the input. Next, the $K_{i}$ values from the distribution were converted to concentration time courses, using pharmacokinetic modeling with the fitted VIF and an assumed $v_{p}$. Noise was added to these curves and the $K_{i}$ and $v_{p}$ measures were recalculated in the same way as the in vivo data for each simulated voxel. This was repeated for scan times increasing from 5 to 25 minutes. We used the SNR (1.68) and $v_{p}(0.005$, comparable to other studies) of the NAWM ${ }^{3,6}$. The NAWM was chosen because BBB leakage is suggested as one of the earlier mechanisms of white matter degeneration, and the measurement of contrast leakage is expected to be more challenging due to the lower blood perfusion compared with the cortex ${ }^{3,28}$. The distributions consisted of $90 \mathrm{~K}$ voxels $\left(180 \mathrm{~cm}^{3}\right)$, which is comparable to the NAWM volume. 
A

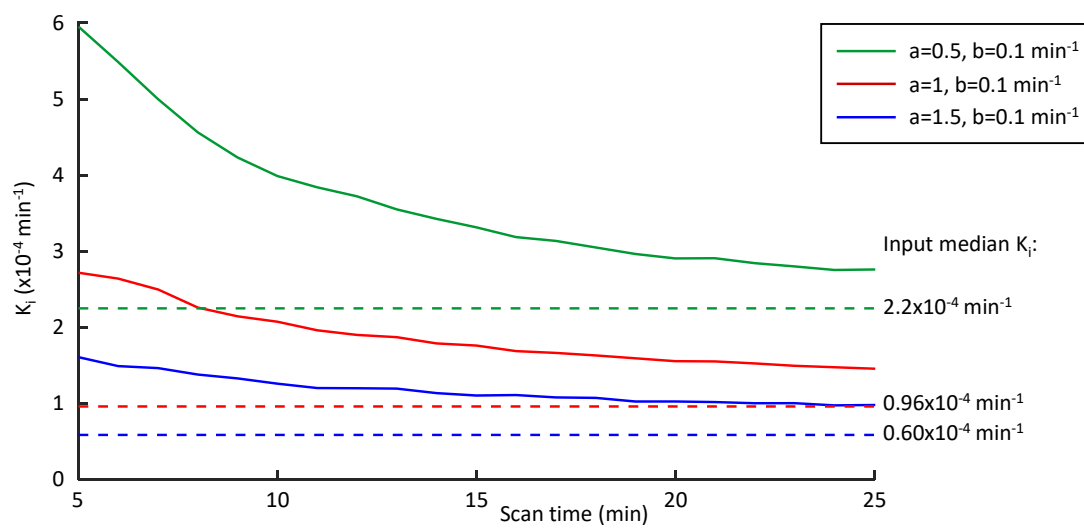

B

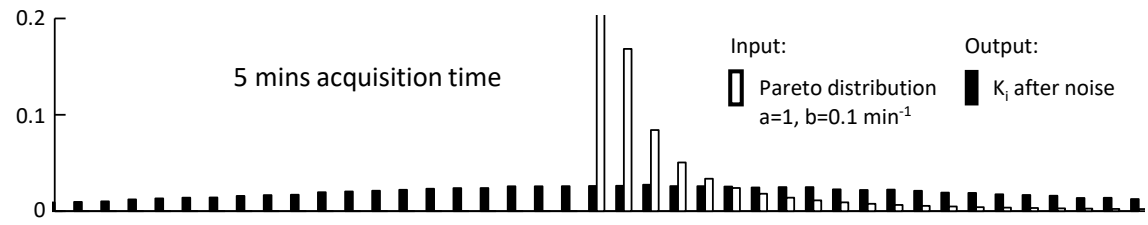

C
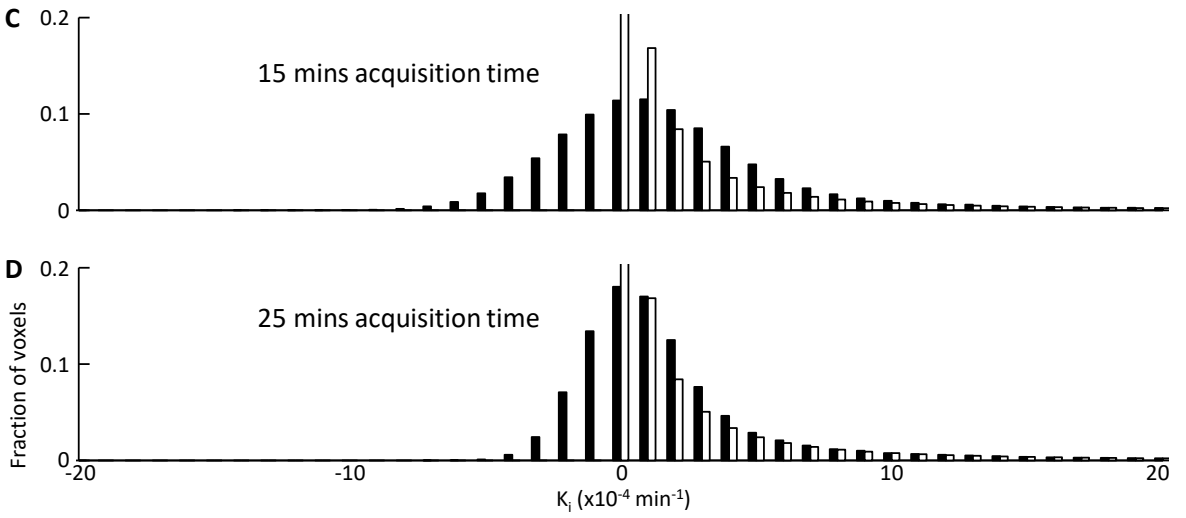

Figure 4.4: The median $\mathrm{K}_{\mathrm{i}}$ of the simulated data versus scan time using different input distribution parameters $(A)$ and $K_{i}$ distributions calculated in the simulations for various scan times $(B, C$ and $D)$. The dashed lines in $A$ indicate the median $K_{i}$ of the profile used as input for the simulations. $K_{i}$ decreases with increasing scan time. The simulations also show that the measured $K_{i}$ is biased, as the input $\mathrm{K}_{\mathrm{i}}$ (dashed lines) is not reached, even after 25 minutes of scan time. This bias is $66 \%$ for the $K_{i}$ profile with the lowest input median (blue) and $23 \%$ for the profile with the highest input median (green). The histograms show the effects of changing the scan time in more detail. The $\mathrm{K}_{\mathrm{i}}$ distribution after noise was added to the concentration-time courses, with a scan time of 5 minutes (B), shows a wide range of $K_{i}$ values. There is no discernable tail at the higher $K_{i}$ values as is shown in the input distribution. A longer scan time $(C)$ reduces the influence of noise and the tail of the $K_{i}$ distribution containina the relevant values becomes better delineated. which continues at lonaer scan times (D). 


\section{Results}

An example of the concentration curves of single voxels with a low and a high $\mathrm{K}_{\mathrm{i}}$, with the corresponding individual VIF and the resulting Patlak plots are shown in Figure 4.5. In the NAWM, the patients had a median $\mathrm{K}_{\mathrm{i}}$ of $0.75 \times 10^{-4}$ (s.d. $0.46 \times 10^{-4}$ ) $\mathrm{min}^{-1}$ and the healthy controls had a slightly lower value of $0.70 \times 10^{-4}$ (s.d. $0.63 \times 10^{-4}$ ) $\mathrm{min}^{-1}$ after 25 minutes of scan time. This difference was not significant $(P=0.8)$. In the cortex, the patients had a higher median $\mathrm{K}_{i}$ of $1.04 \times 10^{-4}$ (s.d. $1.24 \times 10^{-4}$ ) $\mathrm{min}^{-1}$ compared with $0.08 \times 10^{-4}$ (s.d. $0.76 \times 10^{-4}$ ) $\mathrm{min}^{-1}$ in the control subjects after 25 minutes of scan time. This difference was significant $(P=0.014)$, and remained significant until the scan time was shortened to less than 14.5 minutes of scan time (Figure 4.6).

The drift measurements showed a signal decrease of $0.07 \%$ per minute in the $1 \mathrm{mM}$ phantom and a decrease of $0.03 \%$ per minute in the $40 \mathrm{mM}$ phantom. The simulations showed that, assuming an input Pareto distribution with $\mathrm{a}=1$ and $\mathrm{b}=0.1$, this drift results in a relative decrease in $\mathrm{K}_{i}$ of $4.2 \%$ (from $0.96 \times 10^{-4}$ to $0.92 \times 10^{-4} \mathrm{~min}^{-1}$ ), a relative decrease in $V_{L}$ of $7.6 \%$ (from 0.89 to 0.83 ), and no change in $v_{p}(0.005)$.

Both the in vivo measurements and simulations showed that the observed median $\mathrm{K}_{\mathrm{i}}$ will decrease with scan time (Figures 4.4 and 4.6). Further examination of the simulations revealed that at longer scan times, the $K_{i}$ distribution histogram became more skewed towards higher $K_{i}$ values, i.e. the upper tail of the distribution. The decreasing influence of noise results in a decrease of the median $K_{i}$, as less (noisy) voxels are present in the upper tail. The simulations also revealed that even though the measured $K_{i}$ decreases with increasing scan time, the true (i.e. input median) $K_{i}$ is not reached within a scan time of 25 minutes (Figure 4.4). To show the effect of SNR on the $K_{i}$ estimation, we also performed simulations with different SNR but fixed scan time and input $\mathrm{K}_{\mathrm{i}}$. The results of these simulations are displayed in Figure 4.7.

The patients exhibit a significantly higher $\mathrm{V}_{\mathrm{L}}$ compared with the controls in both the NAWM (patients: 0.41 , s.d. 0.17 , controls: 0.27 , s.d. $0.13, p=0.018$ ) and cortical gray matter (patients: 0.31 , s.d. 0.22 , controls: 0.15 , s.d. $0.10, p<0.01$ ) at 25 minutes scan time. We also found that VL decreases with increasing scan time (Figure 4.6).

Leakage maps of a representative patient obtained at different scan times are displayed in Figure 4.8. As can be appreciated from Figure 4.6, 15.5 minutes of total scan time is the minimum to obtain a statistically significant (i.e. $\mathrm{p}<0.05$ ) difference in $\mathrm{vL}$ between 
the patient $(0.52$, s.d. 0.04$)$ and control group $(0.42$, s.d. 0.03$)$ in the NAWM. Only 8 minutes are required in the cortex (patients: 0.58 s.d. 0.06 , controls: 0.42 s.d. 0.04 ), and $P<0.01$ after 12.5 minutes. 
A

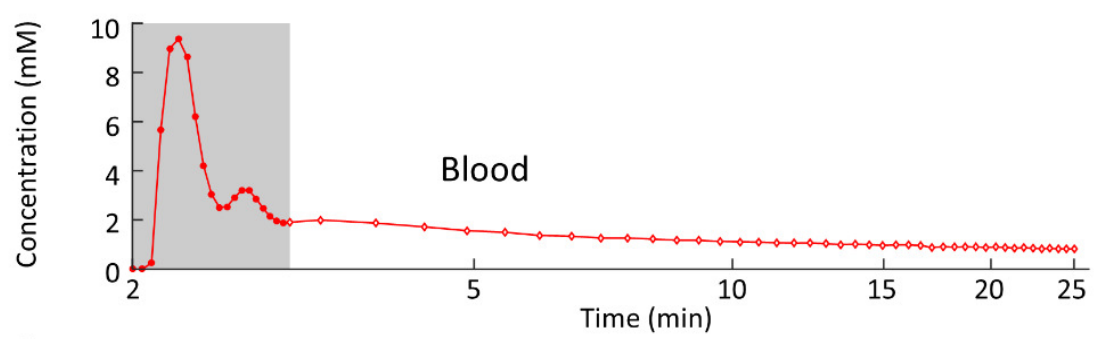

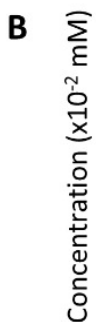

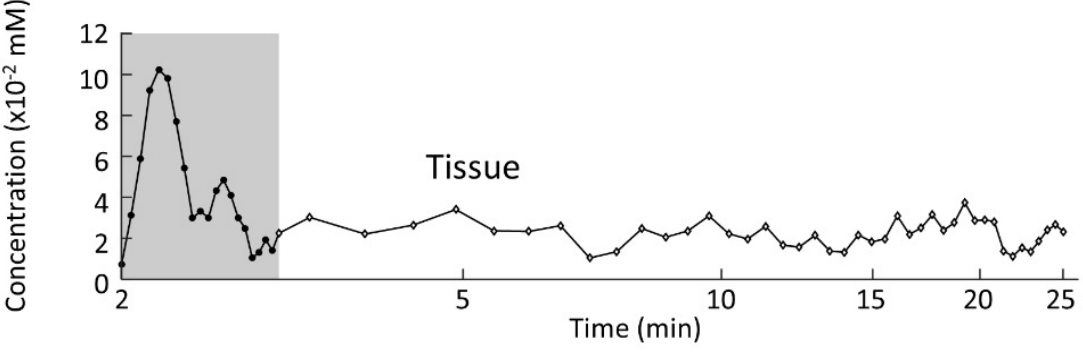

C
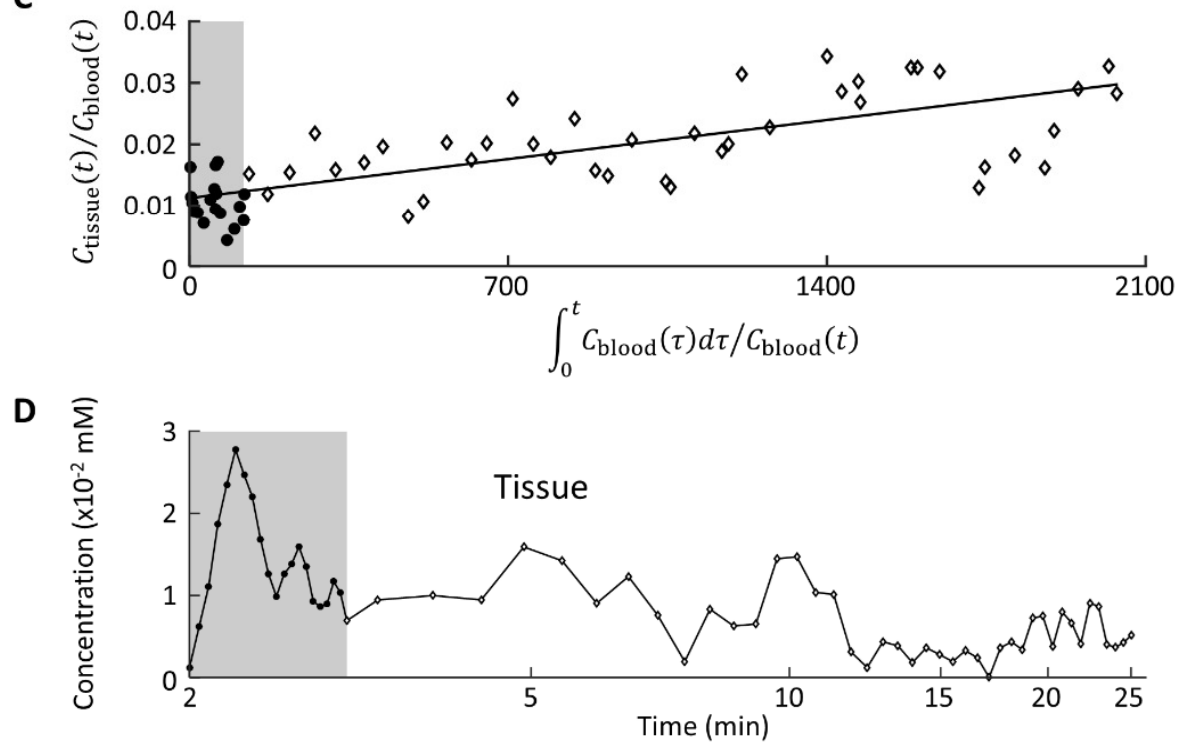

E

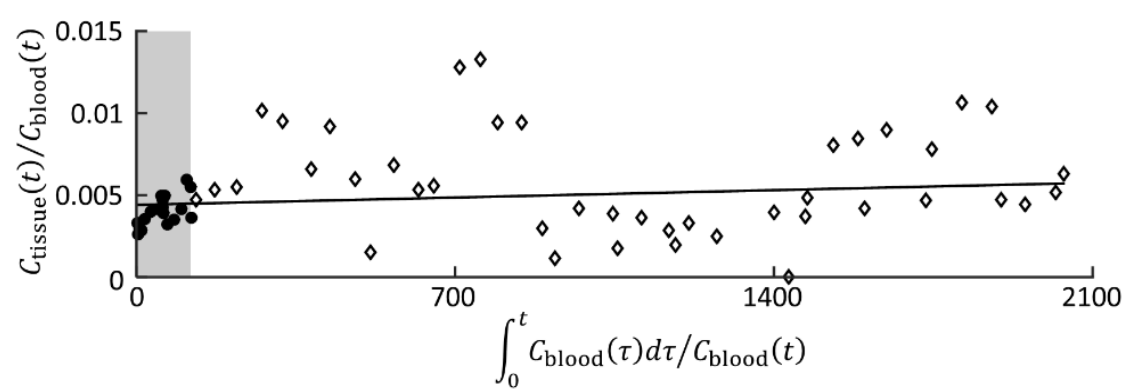


Figure 4.5 (p. 80): Contrast agent concentration time curves in a blood vessel (A) and in tissue (B and $D)$. The Patlak plots resulting from combining the tissue curves with the blood curve, are given $(C$ and $D)$. This resulted in a relatively high $K_{i}(C)$ and a lower $K_{i}$ value $(D)$. The voxels were selected in the normal appearing white matter of a single patient with early Alzheimer's disease. The gray background and filled markers indicate when the fast protocol was used. While the Patlak plot in $\mathrm{C}$ shows a clear upwards trend, resulting in a $\mathrm{K}_{\mathrm{i}}$ of $5.4 \times 10^{-4} \mathrm{~min}^{-1}\left(\right.$ s.e. $1.1 \times 10^{-4} \mathrm{~min}^{-1}$ ) and a $v_{p}=1.1 \%$ (s.e. $0.18 \%$ ), the Patlak plot in $E$ shows a much less coherent pattern, resulting in a $\mathrm{K}_{i} 0.4 \times 10^{-4} \mathrm{~min}^{-1}$ (s.e. $0.34 \times 10^{-4} \mathrm{~min}^{-1}$ ) and $a v_{p}=0.4 \%$ (s.e. $0.06 \%$ ). A slight change in the points of $E$ may cause the slope of the Patlak plot to become negative leading to the measurement of a negative $\mathrm{K}_{\mathrm{i}}$, which illustrates the need for a method to reduce the impact of noise.

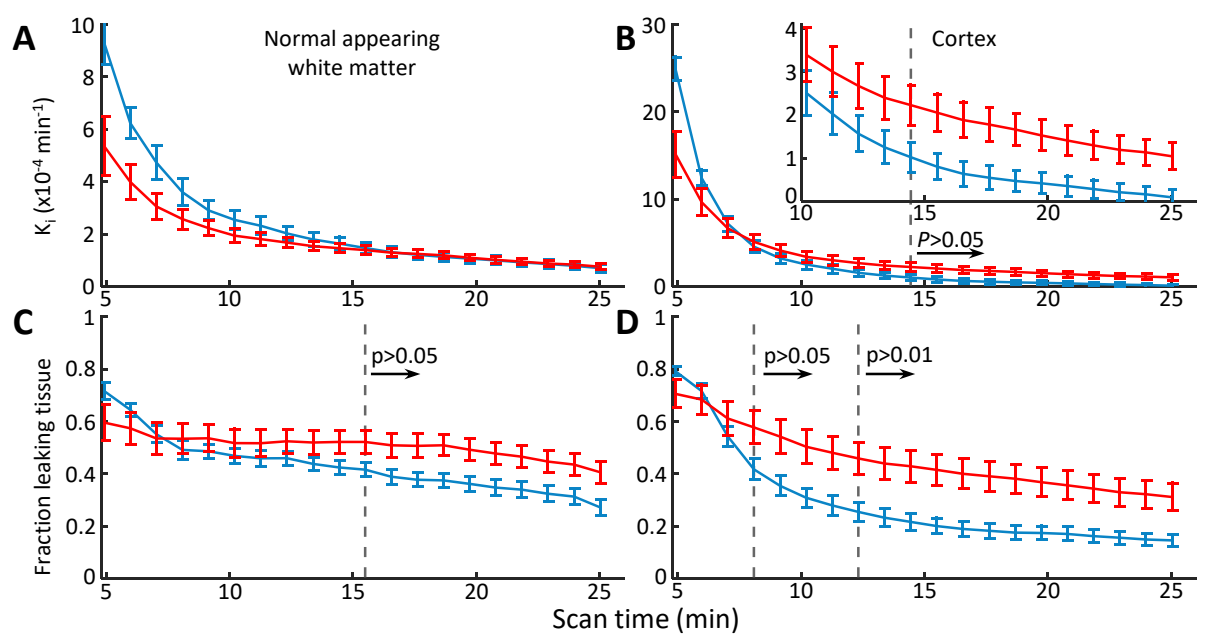

Figure 4.6: Median $\mathrm{K}_{\mathrm{i}}$ of the normal appearing white matter and cortical gray matter of the patients and controls ( $A$ and $B$ ), and fraction leaking tissue $\left(V_{L}\right)(C$ and $D)$ versus scan time. The error bars denote the standard error of the mean. The vertical dashed lines show the scan time at which a significant difference in the fraction of leaking tissue was found between the groups. The second graph in B shows a magnification of the same data in the cortex, to better demonstrate the differences between the groups. As with the simulated data, $K_{i}$ decreases with scan time ( $A$ and $B$ ). Furthermore, $\mathrm{V} L$ also decreases with scan time ( $C$ and $D)$. 
A

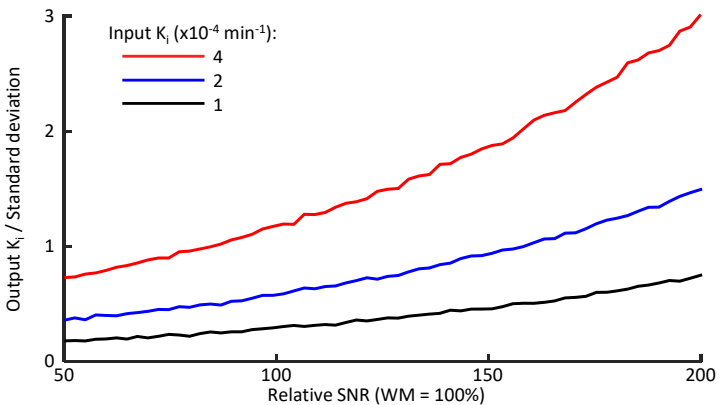

B

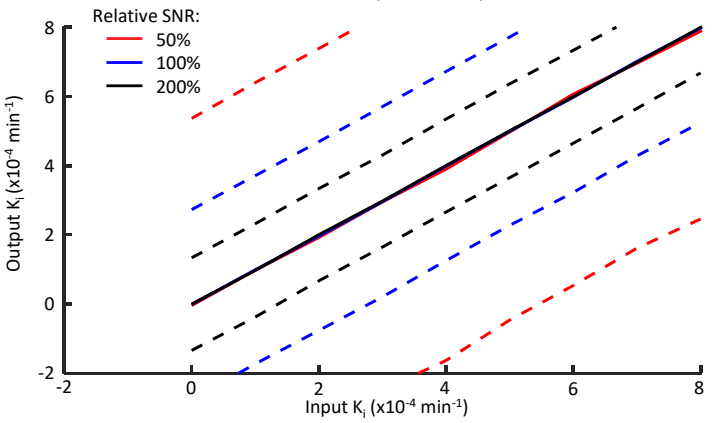

Figure 4.7: Simulations showing the effect of the signal-to-noise ratio (SNR) on the accuracy of the $\mathrm{K}_{\mathrm{i}}$ estimation. The SNR was scaled relative to the SNR found in the white matter (WM) of the in vivo measurement, which was 1.68. Figure $A$ shows the output $K_{i}$ relative to the standard deviation versus the noise level, while $B$ shows the effect of various noise levels on different input $K_{i}$ values.

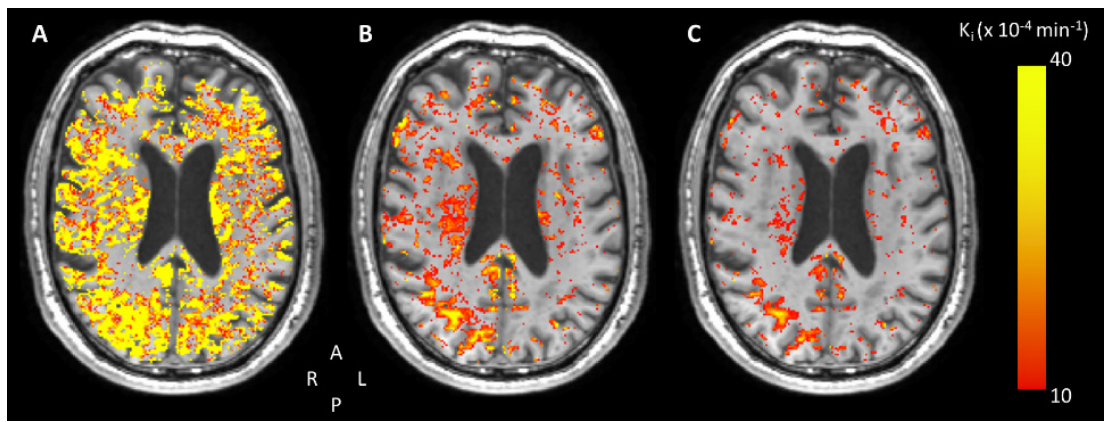

Figure 4.8: Overview of the leakage maps of a patient, superimposed on the T1-weighted image, at 5 (A), 15 (B) and 25 (C) minutes of scan time. Note that voxels with a $K_{i}$ value below the cutoff of $10 \times 10^{-4} \mathrm{~min}^{-1}$ are not shown to focus on the higher and more relevant $\mathrm{K}_{i}$ values. Figure $A$ shows that the voxels have a wide range of $K_{i}$ values at 5 minutes of scan time, but these are heavily influenced by noise. A longer scan time reduces the absolute $K_{i}$ values (as seen by the transition from yellow to red in $B$ ) and less high $K_{i}$ voxels are displayed, while more voxels fall below the threshold of $10 \times 10^{-4}$ $\mathrm{min}^{-1}$. At 25 minutes of scan time $(\mathrm{C})$, the impact of noise is reduced further, and more voxels fall below the display threshold, while higher $\mathrm{K}_{\mathrm{i}}$ voxels become more sparse but also more meaningful. 
A

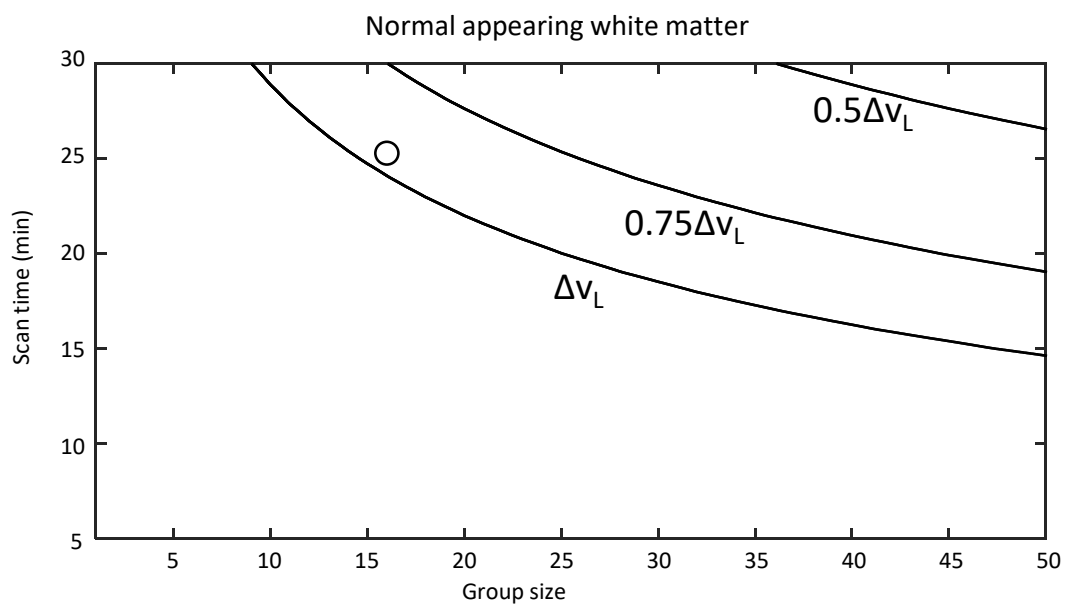

B

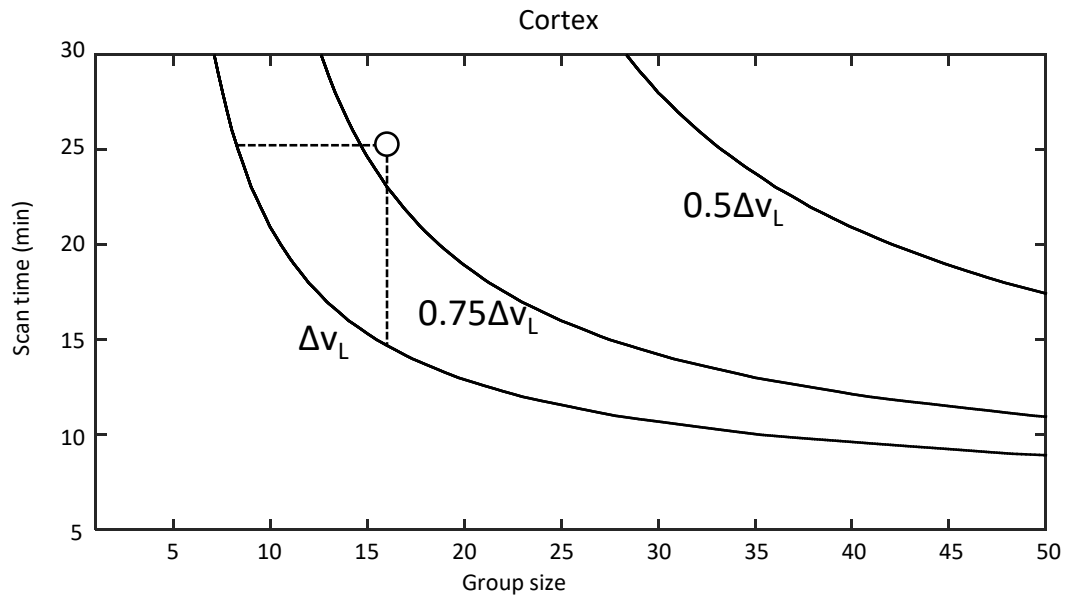

Figure 4.9: The relation between scan time and group size in the normal appearing white matter (NAWM) (A) and cortex (B) at a constant power level of $80 \% . \Delta \mathrm{V}$ is the difference in the fraction of leaking tissue between the patients and the controls. The power contours for different effect sizes are shown $\left(0.5 \Delta V_{L}\right.$ means that the difference between the patients versus the controls is half of the measured difference from the imaging data). The circles denote the current study. The graph shows that $80 \%$ power could still be achieved in the cortex by decreasing the group size to 9 (following the dashed line to the left), or decreasing the scan time to 15 minutes (following the dashed line downwards). However, the current group size and scan time are appropriate if the effect size would be smaller $\left(0.75 \Delta \mathrm{v}_{\mathrm{L}}\right)$ or if, instead of the cortex, the NAWM is of interest. 
Based on the in vivo imaging data, the balance between required group sizes and scan times for a constant statistical power of $80 \%$ was calculated. The depicted relation allows for an estimation of the group sizes and scan time given a certain $\mathrm{K}_{i}$ or $\mathrm{VL}$ difference. The difference in $\mathrm{K}_{i}$ in the NAWM was not significant at any investigated scan time, and the power was quite low (power of $8 \%$ at 25 minutes of scan time and a group size of 16$)$. In the cortex, the $K_{i}$ was significantly different between the groups. The statistical power peaked with $75 \%$ power at 18 minutes of scan time for a group size of 16 (57\% for a scan time of 25 minutes). For the current study, vL proved more sensitive to differences between the groups (Figure 4.9). With 25 minutes of scan time and a group size of 16 , the power of the effect in the NAWM is $85 \%$. In the cortex, the current study reaches a power of $98 \%$. This indicates that the current study had sufficient (statistical) power to find the difference for VL in the NAWM, but shortening the scan time or decreasing the group size would result in the study being underpowered. To measure the effect in the cortex, our data show that $80 \%$ power can still be achieved if the scan time would be lowered to 15 minutes, or the group size would be decreased to 9 (Figure 4.9). However, the current study has $84 \%$ power at $0.75 \Delta \mathrm{V}$, and further shortening of scan time or lowering group size with such an expected effect size will cause the power to fall below the $80 \%$ level.

\section{Discussion}

A number of ways to improve the sensitivity of DCE-MRI to detect widespread subtle BBB leakage were investigated in patients with early $A D$. In vivo imaging data showed that the (median) leakage rate $K_{i}$ is strongly influenced by noise, and that both the observed $K_{i}$ and fraction of leaking tissue $V_{L}$ decrease for longer scan times. Based on the in vivo imaging data, we also calculated the scan time and group size needed for different effect sizes.

DCE-MRI has been used in tumors with success, but applications in neurodegenerative diseases are more difficult. In part, this difficulty can be attributed to the spatial distribution of the leakage. In tumors, increased contrast agent leakage is usually localized in the tumor tissue ${ }^{29}$. However, the leakage may be much more widespread in neurodegenerative diseases. Although voxelwise mapping of leakage may be an appropriate approach to detect widespread leakage, it is also more susceptible to noise compared with region-of-interest approaches. Furthermore, where in tumors the leakage rate may be the most important measure, there is no reason to assume that 
hotspots of high leakage exist in neurodegenerative diseases such as AD. The commonly used mean $\mathrm{K}_{\mathrm{i}}$ in a region may not be the most appropriate measure for an increase in leakage extent rather than rate (i.e. strength). This prompted us to develop the volume of detectable leakage VL. However, future studies may look into other possible ways to measure leakage extent. For example, in functional MRI, approaches based on clustering or independent component analysis have been successfully applied to increase sensitivity to more subtle signal changes due to cortical activation ${ }^{30,31}$. Similar methods may also be useful in DCE-MRI experiments in neurodegenerative diseases.

The effect of noise on the leakage rate distribution changes with scan time, because for longer scan times the impact of noise on the concentration curves is reduced. This is due to the increased number of measurements (time-points) and because the contrast agent has more time to extravasate, resulting in higher extravascular concentrations which are easier to detect. The upper tail of the (skewed) distribution, containing the higher and more relevant voxelwise $K_{i}$ values, becomes more apparent (see also Figure 4.4). Concomitantly, the number of high values that can be attributed to noise are reduced and the noise values in general become more concentrated around zero. On the contrary, for decreasing scan times, the measured concentration-time curves become increasingly dominated by noise, leading to a stronger variation of slope values in the Patlak plots. At shorter scan times the resulting $K_{i}$ histograms are broad and nearly symmetric, with a peak at a positive $K_{i}$ value (due to the skewed input distribution). At such short scan times, actual leakage cannot be distinguished from noise. The skewed input distribution causes the median $K_{i}$ to decrease with longer scan times. The $V L$ also decreases for longer scan times. This is also caused by the decreasing influence of the noisy values in the upper tail of the distribution, causing a larger fraction of voxels to be attributed to noise, lowering the calculated VL.

The subtle leakage profiles found in this study are comparable to previously reported values in neurodegenerative disease, which are as low as $10^{-4} \min ^{-1} 6,12,13,15,32$. The in vivo data show that detecting differences between a group of patients with a neurodegenerative disease and a control group is feasible. However, if the effect is somewhat smaller than what we measured (e.g. $0.75 \Delta \mathrm{V}$ ), the detection of differences becomes more difficult, especially in the white matter. If the difference between the patient and control groups is only half of the original effect $(0.5 \Delta \mathrm{VL})$, the required scan time and group size increase considerably, making such studies more challenging. 
The simulations show an overestimation of $\mathrm{K}_{\mathrm{i}}$, which is still present at scan times up to 25 minutes (Figure 4.4). The median $K_{i}$ observed in the in vivo data is also below the detection limit of $10 \times 10^{-4} \mathrm{~min}^{-1}$ previously reported by Cramer and Larsson ${ }^{6}$. This suggests that determining the absolute $K_{i}$ value seems not feasible given the lower leakage values found in this study. This notion hampers direct comparison of leakage rate values between studies, as the $K_{i}$ values strongly depend on the scan time and noise level. To obtain a representative alternative measure for BBB leakage per participant, we proposed $\mathrm{V} \mathrm{L}$. This measure proved more sensitive to the relatively larger number of subtly leaking voxels in the patients with early $A D$ compared with the controls. Recent studies also emphasized the importance of signal drift on the leakage estimates ${ }^{11,33}$. We found a negative signal drift which, according to the simulations, causes a slight decrease in both $K_{i}$ and $V L$. Because it has such a small influence on the $K_{i}$ and $V L$ and the effect is expected to be similar between the two groups, we did not further correct for it in our analysis.

Given that DCE-MRI is most commonly used in oncology, scan times are often based on much higher $K_{i}$ values than found in neurodegenerative disease. For example, Aerts et al. found that scan times beyond 7 minutes did not further improve leakage estimates in tumor tissue ${ }^{25}$. However, this was established assuming a (tumor) leakage value of 0.1 $\mathrm{min}^{-1}$, which is three orders of magnitude higher than the currently observed values in early $A D\left(K_{i} \approx 1 \times 10^{-4} \mathrm{~min}^{-1}\right)$. A recent study showed the effect of scan time on the contrast-to-noise ratio of the leakage rate $K^{\text {trans }}$ (where $K_{i}=K^{\text {trans }} /(1 \text {-hematocrit })^{11}$. They concluded that the most optimal scan time depended on the value of $K^{\text {trans }}$. Longer scan times provide a more precise value, but also give more time for the contrast agent to return back into the blood (reflux), which is assumed to be negligible in the Patlak model. The leakage rates found in our in vivo data, are much lower than those reported by Barnes et al. (approximately $20 \times 10^{-4} \mathrm{~min}^{-1}$ after hematocrit correction). They reported that such subtle leakage strongly decreases the influence of reflux. On the other hand, the influence of noise becomes quite strong for such low leakage values, which is the central topic of the current study. Cramer and Larsson have also investigated the requirements for measuring subtle BBB leakage, and focused on the pharmacokinetic model and the sampling frequency ${ }^{6}$. Concerning the scan time, they show that increasing the scan time decreases the influence of noise, which manifests itself as a low standard deviation on the estimated $K_{i}$, but has no or negligible impact on the magnitude of $K_{i}$. There are two important methodological aspects worth comparing this study to that of Cramer and Larsson. First, we used a dual time resolution 
DCE-MRI protocol. The high temporal resolution in the study of Cramer and Larsson should prevent undersampling of the VIF, similar to the high temporal resolution part of the dual time resolution protocol ${ }^{16}$. If a lower temporal resolution would be used, the undersampling of the VIF would cause the $\mathrm{K}_{\mathrm{i}}$ to be overestimated, resulting in faulty leakage estimates ${ }^{16,25}$. The dual time resolution sequence also allows for a higher SNR during the long washout of the contrast agent, which may improve the leakage estimation. Second is the use of a $K_{i}$ histogram to simulate the effects of a DCE-MRI experiment, whereas Cramer and Larsson used the more common Monte Carlo approach of single voxel $K_{i}$ values. We feel that the histogram more closely simulates the combination of variations and noise in maps of (very low) $K_{i}$ values, which way provides novel insights into the consequences of varying the scan time.

The necessary scan time can also be influenced by other methodological choices, such as the choice of contrast agent and the pharmacokinetic model ${ }^{6,16,34,35}$. The intrinsic properties of the contrast agent such as lipophilicity, charge and size, determine how easily it may pass the $\mathrm{BBB}^{36}$. If a new contrast agent would be developed that can pass the BBB more easily, the subtle signal differences on MRI would be larger. This would make it easier to measure the leakage, which may translate to a shorter scan time. For the pharmacokinetic model we chose to use the Patlak plot to quantify the leakage, as this was previously shown to be the best approach for subtle leakages when the reflux can be ignored ${ }^{6,11}$. Different models require different scan times, depending on the underlying assumptions of the model ${ }^{11}$. Besides these methodological considerations, it is also important to consider the impact of a long scan time on the subject. It is common that a patient with a neurodegenerative disease has some form of cognitive impairment, which would increase the burden of an MRI experiment compared with a healthy control subject.

In conclusion, we have introduced the leakage volume $\mathrm{V}$, which appears more suitable and time-efficient than leakage rate $K_{i}$ to detect subtle BBB leakage differences between patients with early $A D$ and controls. Compared with $K_{i}, V_{L}$ is a measure for the spatial extent of the leakage rather than the leakage strength. Computer simulations, showing how the measured leakage is affected by changing the scan time, and statistical power calculations were used to help to improve methodological study design. Overall, this information should assist future research aimed at investigating the subtle BBB leakage in neurodegenerative diseases. 


\section{References}

1 Erickson MA, Banks WA. Blood-brain barrier dysfunction as a cause and consequence of Alzheimer's disease. J Cereb Blood Flow Metab 2013; 33: 1500-13.

2 Wardlaw JM, Smith C, Dichgans M. Mechanisms of sporadic cerebral small vessel disease: Insights from neuroimaging. Lancet Neurol 2013; 12: 483-497.

Sourbron S, Ingrisch M, Siefert A, Reiser M, Herrmann K. Quantification of cerebral blood flow, cerebral blood volume, and blood-brain-barrier leakage with DCE-MRI. Magn Reson Med 2009; 62: 205-217.

Zlokovic BV. The Blood-Brain Barrier in Health and Chronic Neurodegenerative Disorders. Neuron 2008; 57: 178-201.

Larsson HBW, Courivaud F, Rostrup E, Hansen AE. Measurement of brain perfusion, blood volume, and blood-brain barrier permeability, using dynamic contrast-enhanced T1-weighted MRI at 3 tesla. Magn Reson Med 2009; 62: 1270-1281.

Cramer SP, Larsson HBW. Accurate determination of blood-brain barrier permeability using dynamic contrast-enhanced T1-weighted MRI: a simulation and in vivo study on healthy subjects and multiple sclerosis patients. J Cereb Blood Flow Metab 2014; : 1-11.

Khalifa F, Soliman A, El-Baz A, Abou El-Ghar M, El-Diasty T, Gimel'farb G et al. Models and methods for analyzing DCE-MRI: A review. Med Phys 2014; 41: 124301.

van de Haar HJ, Burgmans S, Hofman PAM, Verhey FRJ, Jansen JFA, Backes WH. Blood-brain barrier impairment in dementia: Current and future in vivo assessments. Neurosci Biobehav Rev 2015; 49C: 71-81.

Starr JM, Farrall AJ, Armitage P, McGurn B, Wardlaw JM. Blood-brain barrier permeability in Alzheimer's disease: a case-control MRI study. Psychiatry Res - Neuroimaging 2009; 171: 232-241.

Armitage PA, Farrall AJ, Carpenter TK, Doubal FN, Wardlaw JM. Use of dynamic contrast-enhanced MRI to measure subtle blood-brain barrier abnormalities. Magn Reson Imaging 2011; 29: 305-314.

Barnes SR, Ng TSC, Montagne A, Law M, Zlokovic BV., Jacobs RE. Optimal acquisition and modeling parameters for accurate assessment of low $\mathrm{K}$ trans blood-brain barrier permeability using dynamic contrast-enhanced MRI. Magn Reson Med 2016; 75: 1967-1977.

Taheri S, Gasparovic C, Huisa BN, Adair JC, Edmonds E, Prestopnik J et al. Blood-brain barrier permeability abnormalities in vascular cognitive impairment. Stroke 2011; 42: 2158-2163.

Taheri S, Gasparovic C, Shah NJ, Rosenberg G a. Quantitative measurement of blood-brain barrier permeability in human using dynamic contrast-enhanced MRI with fast T1 mapping. Magn Reson Med 2011; 65: 1036-1042.

Bronge L, Wahlund L-OO. Contrast-enhanced MRI of White Matter Lesions in Patients with BloodBrain Barrier Dysfunction. Dement Geriatr Cogn Disord 2000; 11: 477-481.

Montagne A, Barnes SR, Sweeney MD, Halliday MR, Sagare AP, Zhao Z et al. Blood-Brain Barrier Breakdown in the Aging Human Hippocampus. Neuron 2015; 85: 296-302.

Jelescu IO, Leppert IR, Narayanan S, Araújo D, Arnold DL, Pike GB. Dual-temporal resolution dynamic contrast-enhanced MRI protocol for blood-brain barrier permeability measurement in enhancing multiple sclerosis lesions. J Magn Reson Imaging 2011; 33: 1291-1300.

Fischl B, Salat DH, Busa E, Albert M, Dieterich M, Haselgrove C et al. Whole brain segmentation: Automated labeling of neuroanatomical structures in the human brain. Neuron 2002; 33: 341-355. 
de Boer R, Vrooman HA., van der Lijn F, Vernooij MW, Ikram MA, van der Lugt A et al. White matter lesion extension to automatic brain tissue segmentation on MRI. Neuroimage 2009; 45: 1151-1161.

Haroon HA, Buckley DL, Patankar TA, Dow GR, Rutherford SA, Balériaux D et al. A Comparison of Ktrans Measurements Obtained with Conventional and First Pass Pharmacokinetic Models in Human Gliomas. J Magn Reson Imaging 2004; 19: 527-536.

Li KL, Zhu XP, Waterton J, Jackson a. Improved 3D quantitative mapping of blood volume and endothelial permeability in brain tumors. J Magn Reson Imaging 2000; 12: 347-357.

Lavini C, Verhoeff JJC. Reproducibility of the gadolinium concentration measurements and of the fitting parameters of the vascular input function in the superior sagittal sinus in a patient population. Magn Reson Imaging 2010; 28: 1420-1430.

Pintaske J, Martirosian P, Graf H, Erb G, Lodemann K-P, Claussen CD et al. Relaxivity of Gadopentetate Dimeglumine (Magnevist), Gadobutrol (Gadovist), and Gadobenate Dimeglumine (MultiHance) in human blood plasma at 0.2, 1.5, and 3 Tesla. Invest Radiol 2006; 41: 213-221.

Schabel MC, Parker DL. Uncertainty and bias in contrast concentration measurements using spoiled gradient echo pulse sequences. Phys Med Biol 2008; 53: 2345-2373.

Patlak CS, Blasberg RG, Fenstermacher JD. Graphical evaluation of blood-to-brain transfer constants from multiple-time uptake data. J Cereb Blood Flow Metab 1983; 3: 1-7.

Aerts HJWL, Jaspers K, Backes WH. The precision of pharmacokinetic parameters in dynamic contrast-enhanced magnetic resonance imaging: the effect of sampling frequency and duration. Phys Med Biol 2011; 56: 5665-5678.

Zar JH. Biostatistical Analysis. 4th ed. Prentice Hall, 1999.

McGrath DM, Bradley DP, Tessier JL, Lacey T, Taylor CJ, Parker GJM. Comparison of model-based arterial input functions for dynamic contrast-enhanced MRI in tumor bearing rats. Magn Reson Med 2009; 61: 1173-1184.

Wardlaw JM, Sandercock PAG, Dennis MS, Starr J. Is breakdown of the blood-brain barrier responsible for lacunar stroke, leukoaraiosis, and dementia? Stroke 2003; 34: 806-811.

Choyke PL, Dwyer AJ, Knopp M V. Functional tumor imaging with dynamic contrast-enhanced magnetic resonance imaging. J Magn Reson Imaging 2003; 17: 509-520.

Lee $\mathrm{MH}$, Smyser $\mathrm{CD}$, Shimony JS. Resting-state $\mathrm{fMRI}$ : a review of methods and clinical applications. Am J Neuroradiol 2013; 34: 1866-1872.

Woo C-W, Krishnan A, Wager TD. Cluster-extent based thresholding in fMRI analyses: Pitfalls and recommendations. Neuroimage 2014; 91: 412-419.

Heye AK, Culling RD, Valdés Hernández MDC, Thrippleton MJ, Wardlaw JM. Assessment of bloodbrain barrier disruption using dynamic contrast-enhanced MRI. A systematic review. Neurolmage Clin 2014; 6: 262-74.

Heye AK, Thrippleton MJ, Armitage PA, Valdés Hernández M del C, Makin SD, Glatz A et al. Tracer kinetic modelling for DCE-MRI quantification of subtle blood-brain barrier permeability. Neuroimage 2016; 125: 446-455.

Cao Y, Li D, Shen Z, Normolle D. Sensitivity of Quantitative Metrics Derived from DCE MRI and a Pharmacokinetic Model to Image Quality and Acquisition Parameters. Acad Radiol 2010; 17: 468478.

Kershaw LE, Cheng HLM. Temporal resolution and SNR requirements for accurate DCE-MRI data analysis using the AATH model. Magn Reson Med 2010; 64: 1772-1780. 
Chapter 4

36

Seelig A, Gottschlich R, Devant RM. A method to determine the ability of drugs to diffuse through the blood-brain barrier. Proc Natl Acad Sci U S A 1994; 91: 68-72. 


\section{Chapter 5}

Neurovascular unit impairment in early Alzheimer's disease measured with MRI

Harm J van de Haar, Jacobus FA Jansen, Matthias JP van Osch, Mark A van Buchem, Majon Muller, Sau May Wong, Paul AM Hofman, Saartje Burgmans, Frans RJ Verhey, Walter H Backes Neurobiology of Aging, in press 


\section{Abstract}

The neurovascular unit, which protects neuronal cells and supplies them with essential molecules, plays an important role in the pathophysiology of AD. The aim of this study was to noninvasively investigate two linked functional elements of the neurovascular unit, blood-brain barrier (BBB) permeability and cerebral blood flow (CBF), in patients with early $A D$ and healthy controls. Therefore, both dynamic contrast-enhanced $M R I$ and arterial spin labeling MRI were applied to measure BBB permeability and CBF, respectively. The patients with early $A D$ showed significantly lower CBF and local blood volume in the gray matter, compared with controls. In the patients, we also found that a reduction in $\mathrm{CBF}$ is correlated with an increase in leakage rate. This finding supports the hypothesis that neurovascular damage, and in particular impairment of the neurovascular unit constitutes the pathophysiological link between CBF reduction and BBB impairment in AD. 


\section{Introduction}

The pathogenesis of Alzheimer's Disease (AD) is still unknown, despite decades of research. A popular hypothesis states that the accumulation of insoluble proteins is a central part of the pathological cascade that leads to $A D^{1}$. However, there is also very strong evidence that cerebrovascular damage is a key element in this cascade ${ }^{2}$. The notion that cerebrovascular pathology contributes to $A D$ has recently gained more popularity, as the amyloid hypothesis does not satisfactory represents the cause of the disease $^{3}$. Therefore, assessment of the brain microvasculature in the brain is of particular interest in $A D$.

Previously, it has been hypothesized that hypoperfusion and blood-brain barrier (BBB) impairment in $A D$ are both integral parts of the pathological cascade leading to $A D^{4,5}$. This hypothesis is centered around the neurovascular unit, a collection of capillary endothelial cells and smooth muscle cells, neuronal cells, neurons and subcellular structures in the brain ${ }^{6}$. Different parts of the neurovascular unit form functional elements, which work together to perform necessary functions. One of these functions is that the BBB protects the brain from circulating neurotoxins while allowing molecules essential to the parenchyma to pass ${ }^{7,8}$. Another important function of the neurovascular unit is the regulation of blood flow.

A general hypothesis is that impairment of the neurovascular unit is a central feature in the pathophysiology that leads to AD. In short, the neurovascular unit hypothesis states that any damage to the neurovascular unit can start a complicated cascade involving a reduced cerebral blood flow (CBF) and BBB disruption ${ }^{9-13}$. This is a self-reinforcing loop which triggers other damaging responses including inflammation, oxidative stress and upregulation of vasoconstrictors ${ }^{4,9,14,15}$. It is also known that hypoperfusion is correlated with dementia severity ${ }^{16,17}$, indicating that, over time, the neurovascular unit impairment increases and chronic damage results in neurodegeneration and cognitive decline. The neurovascular unit has been found to be impaired in dementia, mainly due to inflammation, oxidative stress and a nitric oxide deficit, leading to reduced cerebral blood flow (CBF) and increased BBB permeability ${ }^{10,14,18}$. Given the gradual progression of $A D$, this impairment is expected to start early in the disease process. Furthermore, under the assumption that this loop is an integral part of the pathophysiology of early $A D$, the impairment of the different functional elements of the neurovascular unit are expected to co-occur, and thus may be correlated. Thus far, these mechanisms are, to 
a large extent, deduced from animal and in vitro studies ${ }^{12}$. However, in vivo human studies are scarce.

If there is widespread damage to the neurovascular unit, global deterioration of measures that should be strictly regulated by the neurovascular unit can be expected. Advanced MRI techniques allow for spatially resolved measurement of some of these measures that are relevant to $A D$, namely the $C B F$, BBB leakage and local blood volume. In this study, we measured CBF with arterial spin labeling (ASL), which magnetically labels protons of the arterial blood and subsequently follows their distribution throughout the brain ${ }^{19}$. These CBF measurements were combined with a dedicated dynamic contrast-enhanced (DCE-) MRI protocol which allows for the measurement of subtle BBB leakage. With DCE-MRI the spatiotemporal distribution of a contrast agent over brain vasculature and parenchyma is measured. It is a suitable method to examine local cerebral blood plasma volume $\left(\mathrm{V}_{\mathrm{p}}\right)$ and the leakage rate $\left(\mathrm{K}_{\mathrm{i}}\right.$, in $\left.\mathrm{min}^{-1}\right)$ of a contrast agent from the blood space into the parenchyma ${ }^{20,21}$.

Our aim was to investigate the functioning of neurovascular unit in terms of blood flow regulation and $B B B$ function in $A D$ by examining the possible link between cerebral (microvascular) perfusion and BBB leakage in the gray matter of patients with early $A D$ and healthy controls.

\section{Materials and Methods}

\section{Participants}

Patients were recruited at the memory clinics from the Maastricht University Medical Center (MUMC) and Leiden University Medical Center (LUMC). All patients were clinically diagnosed with either mild cognitive impairment $(\mathrm{MCl})$ due to $A D$ or mild dementia of the Alzheimer type, which we combined into one group and termed early AD. Diagnosis was made by consensus of a multidisciplinary team according to the Dubois criteria for $\mathrm{MCl}$ and the criteria of the National Institute on Aging and the Alzheimer's Association for $A D^{22,23}$. None of the patients had an evident cerebrovascular origin for the dementia such as a history of stroke. In addition, participants were excluded in case of contraindications for MRI, renal dysfunction, major structural brain abnormalities such as tumors, alcohol/drug abuse, cerebrovascular pathology, or other major psychiatric or neurological disorders. The controls were recruited through advertisements in local newspapers. All participants underwent the mini mental state examination (MMSE) test 
before $M R I^{24}$. This study was approved by both the Medical Ethical Committee AZM/UM and the Committee for Medical Ethics LUMC. Written informed consent was obtained from all participants. We included 16 patients and 18 healthy controls ( 4 and 3 at the LUMC, respectively). Two patients were excluded because of incomplete MRI examinations and 2 controls were excluded because of severe motion induced artefacts and a lowered renal function. Therefore, the data of 14 patients $(7 \mathrm{AD}, 7 \mathrm{MCl}$, mean age $75.3 \mathrm{y}$, range 65 - $85 \mathrm{y}, 8$ males) and 16 healthy controls (mean age $76.4 \mathrm{y}$, range 65 85 y, 11 males) was used for final analysis.

\section{Image acquisition}

CBF was measured using a pseudo-continuous (PC)ASL 2D multislice sequence with a TR/TE of $3847 / 14 \mathrm{~ms}$, voxel size of $3 \times 3 \times 7 \mathrm{~mm}^{3}$, matrix size of $80 \times 80 \times 17$, a $1525 \mathrm{~ms}$ postlabeling delay (PLD) of the most inferior slice, and a label duration of $1650 \mathrm{~ms}$. Slices were obtained in the inferior-superior direction ( $35 \mathrm{~ms}$ per slice), and 50 controllabel pairs were acquired. The labeling slice was positioned perpendicular to the internal carotid arteries, with the help of coronally and sagittally oriented phase contrast angiograms. A single proton density (PD) sequence was acquired with the same geometric properties as the PCASL sequence and a TR of $10 \mathrm{~s}$ to scale the PCASL signal intensity to absolute $\mathrm{CBF}$ in $\mathrm{ml} / \mathrm{min} / 100 \mathrm{~g}$ of brain tissue.

Blood-brain barrier leakage and local blood plasma volume were measured using a dualtime resolution DCE-MRI sequence, which was developed to increase sensitivity to the subtle leakage expected in early AD. This protocol utilizes a high temporal resolution during contrast agent arrival and initial distribution to properly sample the initial arrival peak (the fast sequence), and a lower temporal but higher signal-to-noise during the longer washout (the slow sequence) ${ }^{25}$. The acquisition protocol and analysis method are analogous to a previously described procedure ${ }^{26}$. The fast sequence was a 3D saturation recovery gradient recalled sequence with a TR/TE $5.2 / 2.5 \mathrm{~ms}$, flip angle $30^{\circ}$, voxel size $1 \times 1 \times 5 \mathrm{~mm}^{3}$, matrix size $256 \times 200 \times 10$, SENSE factor 2 (direction RL), with a saturation prepulse given at a delay time (TD) of $120 \mathrm{~ms}, 29$ volumes total, dynamic scan interval (DSI) of $3.2 \mathrm{~s}$ resulting in a scan time of 1.55 minutes. The contrast agent was injected at the fourth dynamic scan (gadobutrol, $0.1 \mathrm{mmol} / \mathrm{kg}$, injected using a power injector with a flow of $3 \mathrm{ml} / \mathrm{s}$, followed by a $20 \mathrm{ml}$ saline flush). The slow sequence was a saturation recovery gradient recalled sequence (TR/TE $5.6 / 2.5 \mathrm{~ms}$, flip angle $30^{\circ}$, voxel size $1 \times 1 \times 2 \mathrm{~mm}^{3}$, matrix size 256×256x50 matrix, SENSE factor 2 (direction RL), DSI 31.8 s, 45 volumes (including 3 precontrast volumes), with the same prepulse. The slow 
sequence started immediately after the fast sequence, for a total scan time of 25 minutes. The field-of-view (FOV) was centered on the corpus callosum, ensuring optimal coverage of the brain surrounding the lateral ventricles, which is the most common location of white matter hyperintensities. Since the white matter hyperintensities are presumed to be of vascular origin, we expected the periventricular region to show the most microvascular pathology ${ }^{27}$. The two sequences were combined by upsampling the volumes of the fast sequence using linear interpolation to match the slow sequence FOV and voxel size, although it should be noted that this posed a limit on the final resolution.

A T1-weighted structural scan (TR/TI/TE of $8.4 / 706 / 4.0 \mathrm{~ms}$, flip angle of $8^{\circ}$, cubic voxel size of $1 \mathrm{~mm}$, matrix size $256 \times 155 \times 256$ ) was used for structural reference and to perform image registration between the various contrasts. All imaging was performed on a dualtransmit 3 Tesla system (Philips Achieva, Best, the Netherlands), with a 32-channel receiver head coil.

\section{Image analysis}

Motion correction for all sequences was performed using FMRIB's linear image registration tool (FLIRT ${ }^{28}$ ) using a mutual-information algorithm. Further analysis was performed by a researcher blinded to the using in-house developed software implemented in Matlab (version 2012b MathWorks, Natick, MA, USA).

\section{Arterial Spin Labeling MRI}

Motion correction was performed relative to the mean of the control images. Next, the label images were subtracted from the control images. Control-label pairs were removed when visual inspection of the subtraction result showed artefacts. CBF calculation was based on an adaptation of the formula proposed in the ASL whitepaper presented by the ISMRM Perfusion Study Group and the European Consortium for ASL in Dementia ${ }^{19}$ to correct for the 2D multislice readout scheme and by including a correction factor for background suppression ${ }^{29}$ :

$$
\mathrm{CBF}=\frac{6000 \cdot \lambda \cdot\left(\mathrm{SI}_{\text {control }}-\mathrm{SI}_{\text {label }}\right) \cdot e^{T_{\text {delay }}+T_{\text {slice }}(z-1) / T_{1, \text { blood }}}}{2 \cdot \alpha \cdot \alpha_{\text {inv }} \cdot T_{1, \text { blood }} \cdot \mathrm{SI}_{\mathrm{PD}} \cdot\left(1-\mathrm{e}^{-\tau / T_{1, \text { blood }}}\right)}
$$

Here $\lambda$ is the blood-brain partition coefficient (set at $0.9 \mathrm{ml} / \mathrm{g}^{30}$ ), Sl control and Slabel are the means over time of the control and label images, respectively, $T_{\text {delay }}$ is the post label delay $(1525 \mathrm{~ms}), T_{\text {slice }}$ is the acquisition time for a single slice $(35 \mathrm{~ms}), z$ is the slice 96 
number, $T_{1}$,blood is the longitudinal relaxation time of blood (set at $1650 \mathrm{~ms}$ for $3 \mathrm{~T}^{31}$ ), $a$ is the labeling efficiency (set at $0.85^{19}$ ), $a_{\text {inv }}$ is a correction factor for the background suppression (set at $0.83^{29}$ ), SlPD is the signal intensity of the proton density image and $\tau$ is the label duration (1650 ms). This study will focus on effects in the gray matter (GM), as ASL in the white matter (WM) remains challenging ${ }^{29}$.

\section{Dynamic contrast-enhanced MRI}

Motion correction was performed relative to the mean of the precontrast images. Individual vascular input functions (VIF) were extracted from the superior sagittal sinus, which was chosen as this was the largest cerebral blood vessel in the FOV, and has been used successfully in other studies ${ }^{25,32-34}$. Translation of signal enhancement to contrast agent concentration was performed differently for the VIF and for tissue. The conversion of signal of the VIF to contrast agent concentration was implemented using an in vitro diluted $\mathrm{MnCl} 2$ stock solution with different gadobutrol concentrations (range 0-40 mM, baseline T1 relaxation time of $1650 \mathrm{~ms}$, comparable to human blood). For tissue, we observed much lower signal changes. Therefore, a linear relationship between signal change and contrast agent concentration $\left(C_{t}\right)$ was assumed for tissue, using ${ }^{35}: C_{t}(t)=$ $\frac{S(t) / S_{0}-1}{T 10 \cdot r_{1}}$ with a contrast agent relaxivity $r_{1}$ of $3.3 \mathrm{~s}^{-1} \mathrm{mM}^{-1}$ and $S_{0}$ as the precontrast signal intensity ${ }^{36}$. The range of contrast agent concentration found in brain tissue was approximately 0-0.2 $\mathrm{mM}$. At $0.2 \mathrm{mM}$, the linear approximation showed an error margin of $2.2 \%$. For this calculation baseline T10 values were used, based on measurements using a pulse sequence comparable to the slow sequence but with different TD values (120-4000 ms) using $S=M_{0} \cdot\left(1-e^{-T D / T 10}\right)$ where $S$ is the measured signal and $M_{0}$ is a fit parameter in which the magnetization and scanner-specific properties are combined ${ }^{37}$. The mean T10 values for the tissue of the participants was calculated, and the T1 structural scans were used to assign voxelwise T10 values. These T10 maps were smoothed with a $2 \times 2 \times 2 \mathrm{~mm}$ kernel. We also measured signal drift by using the total DCEMRI sequence on phantoms with two different gadobutrol concentrations ( 1 and $40 \mathrm{mM}$ ). After calculation of the concentration-time courses, the BBB leakage rate $K_{i}$ (in $\min ^{-1}$ ) and local blood plasma volume $v_{p}$ (fraction of total voxel volume) were determined using the Patlak graphical approach using ${ }^{38}$ :

$$
C_{t}(t)=v_{p} C_{p}(t)+K_{i}(1-\mathrm{Hct}) \int_{0}^{t} C_{p}(\tau) d \tau
$$


Where Hct is the hematocrit, which was set to $45 \%$ and $C_{p}$ and $C_{t}$ are the contrast agent concentration in blood plasma and tissue, respectively. Next, the mean $K_{i}$ and $v_{p}$ were calculated per participant and used for statistical analysis.

The noise on the concentration curves sometimes caused negative slope values in the Patlak plot. To further increase the sensitivity of this leakage detection for the lowest possible $K_{i}$ values close to the noise level, a histogram approach was used. The histograms were normalized and noise was estimated by assuming that negative slope values can only be attributed to noise, and that a similar distribution of noise is also present in positive slope values. The data were then corrected by subtracting the estimated noise from the measured histogram. The remaining cumulative sum of the bins was defined as the BBB leakage volume fraction $V_{L}$. For further details on this procedure, we refer to our previous work ${ }^{26}$. Note that the noise suppression method was only used to calculate $\mathrm{VL}$, and when the $\mathrm{K}_{\mathrm{i}}$ is reported, the mean of the entire histogram is meant without applying the noise suppression method.

\section{Structural reference}

The T1-weighted structural scan was processed using the FreeSurfer software package to automatically define the gray matter ${ }^{39}$. This software was also used to calculate an atrophy score per participant (atrophy score $=1-(G M$ volume + WM volume) $/$ intracranial volume). Next, the mean values of $\mathrm{CBF}, \mathrm{K}_{\mathrm{i}}$, and $\mathrm{v}_{\mathrm{p}}$ of the total $\mathrm{GM}$ were calculated per participant. As the ASL images had the lowest spatial resolution, it was chosen as a reference, and the T1-weighted image and the DCE-MRI images were linearly transformed to the ASL images to calculate the correlation between CBF and the DCE measures.

\section{Statistics}

All statistical tests were performed using SPSS (IBM SPSS Statistics for Windows, Version 20.0, NY, United States). The difference in CBF, $K_{i}, V_{L}$, and $v_{p}$ in the GM between the patients and controls, corrected for age and gender, was first tested using linear regression. Next, the Pearson correlation coefficient was calculated between the GM $\mathrm{CBF}$ and the DCE measures in the patient and control groups. Because the effect of age and atrophy may influence these correlations, they were subsequently added as covariates in a multivariable regression analysis. To find any regional differences, the group comparison analysis was repeated for different gray matter regions, the frontal, temporal parietal and occipital cortex and the deep (subcortical) GM. To correct for the 
increasing number of statistical tests in the regional analyses, a false discovery rate $(F D R)$ procedure for multiple comparisons $(q=0.05)$ was applied. Statistical significance was inferred when $\mathrm{p}<0.05$.

\section{Results}

The patients had a score $\leq 2$ on the modified Hachinski scale, indicating that the dementia did not have a vascular origin ${ }^{40}$. All patients diagnosed with $A D$ had a Clinical Dementia Rating (CDR) of 1 , indicating a mild stage of dementia ${ }^{41,42}$. MRI revealed that the patients had a mean medial temporal lobe atrophy (MTA) score of 1.6 (s.d. 1.0) ${ }^{43}$ and a mean Fazekas score of 1.9 (range $0-3$, controls: 1.4 , range $0-3, p=0.12)^{44}$. The incidence of other vascular risk factors, including atherosclerosis, cardiac arrhythmia and coronary disease, was not significantly different between the groups (patients: 5 , controls: $2, p=0.14)$. On neuropsychological tests, the patients had a mean digit span Wechsler adult intelligence scale (WAIS-III) test score of 11.9 (s.d. 2.0) ) $^{45}$, and a mean letter digit substitution test (LDST, correct items after $90 \mathrm{~s}$ ) score of 29.5 (s.d. 12.7) correct and 0.1 (s.d. 0.3) incorrect ${ }^{46}$.

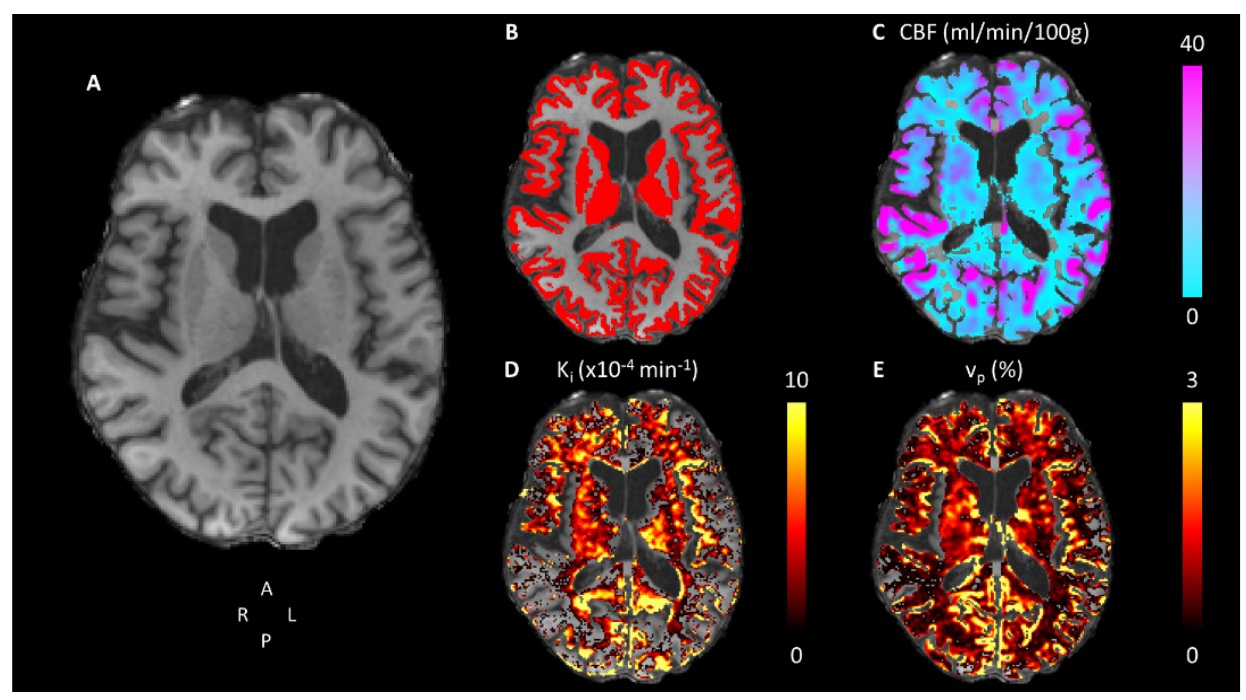

Figure 5.1: A T1-weighted structural image for anatomical reference $(A)$, and the gray matter segmentation as overlay on the T1-weighted image (B). Maps showing the cerebral blood flow measured using arterial spin labeling $(C)$, the dynamic contrast enhanced MRI measures blood-brain barrier leakage rate $K_{i}(D)$ and blood plasma volume $v_{p}(E)$ as color-coded overlays on this T1weighted image. All images are from the same patient with early Alzheimer's disease. The maps show all values greater than zero and were masked to the brain (gray and white matter) tissue. 
Example maps of the CBF, $K_{i}$ and $v_{p}$ measures can be seen in Figure 5.1. To demonstrate the quality of the data, example concentration-time curves and a Patlak plot are shown in Figure 5.2. An overview of the results of the CBF, $K_{i}, V_{L}$ and $v_{p}$ values in the $G M$ is displayed in Figure 5.3. The mean CBF in the GM was significantly lower in the patients (32.1 s.d. $7.0 \mathrm{ml} / \mathrm{min} / 100 \mathrm{~g}$ ) compared with the controls $(39.3 \mathrm{s.d} .6 .4 \mathrm{ml} / \mathrm{min} / 100 \mathrm{~g}$, $p<0.001$, also see Table 5.1). The mean $K_{i}$ value in the GM showed a trend of a higher $K_{i}$ in the patients $\left(2.7 \times 10^{-4}\right.$ s.d. $\left.1.4 \times 10^{-4} \mathrm{~min}^{-1}\right)$ compared with controls $\left(1.8 \times 10^{-4}\right.$ s.d., $\left.1.3 \times 10^{-4} \mathrm{~min}^{-1}, \mathrm{p}=0.055\right)$. The $\mathrm{VL}$ was significantly higher in patients $(0.38 \mathrm{s.d}$. 0.29) compared with controls $(0.12$ s.d. $0.10, p=0.001)$. The $v_{p}$ in the $G M$ was significantly lower in the patients $(1.5$ s.d. $0.5 \%)$ compared with controls $(2.0$ s.d. $0.3 \%, p<0.001)$. The atrophy score was not significantly different between the patients (34.4\% s.d. $7.3 \%$ ) and controls $(32.7 \%$ s.d. $3.4 \%, p=0.4)$. Linear regression revealed a significant negative correlation between CBF and $K_{i}$ in the GM of the patients (Pearson's $r=-0.69, p=0.007$, also see Figure 5.4). There was no significant correlation between CBF and $K_{i}$ in the controls $(p=0.6)$. Between $C B F$ and $V_{L}$, a significant correlation was found in the $G M$ of the patients (Pearson's $r=-0.54, p<0.05$, Figure 5.4), but not for the controls $(p=0.6)$. There were no significant correlations between CBF and $v_{p}$ for the patients $(p=0.4)$ or the controls $(p=0.9)$. Adding age and atrophy as potential confounders to these correlations had a minor effect on the correlation between CBF and $K_{i}$ (Pearson's $r=-$ 0.73, $p=0.011$ ), and for the correlation between $C B F$ and $V_{L}$ it raised the $p$ value to 0.07 (Pearson's $r=-0.57$ ), making it a trend. The results of the regional CBF are listed in Table 5.1. In short, all regions exhibit significant hypoperfusion in the patients compared with the controls, which remains after FDR correction. The drift measurements showed a signal decrease of $0.07 \%$ per minute in the low concentration $(1 \mathrm{mM})$ phantom and a decrease of $0.03 \%$ per minute in the high concentration $(40 \mathrm{mM})$ phantom. This causes a decrease in $\mathrm{K}_{\mathrm{i}}$ (5\% in patients and $9 \%$ in controls) and no change in $\mathrm{v}_{\mathrm{p}}$. 

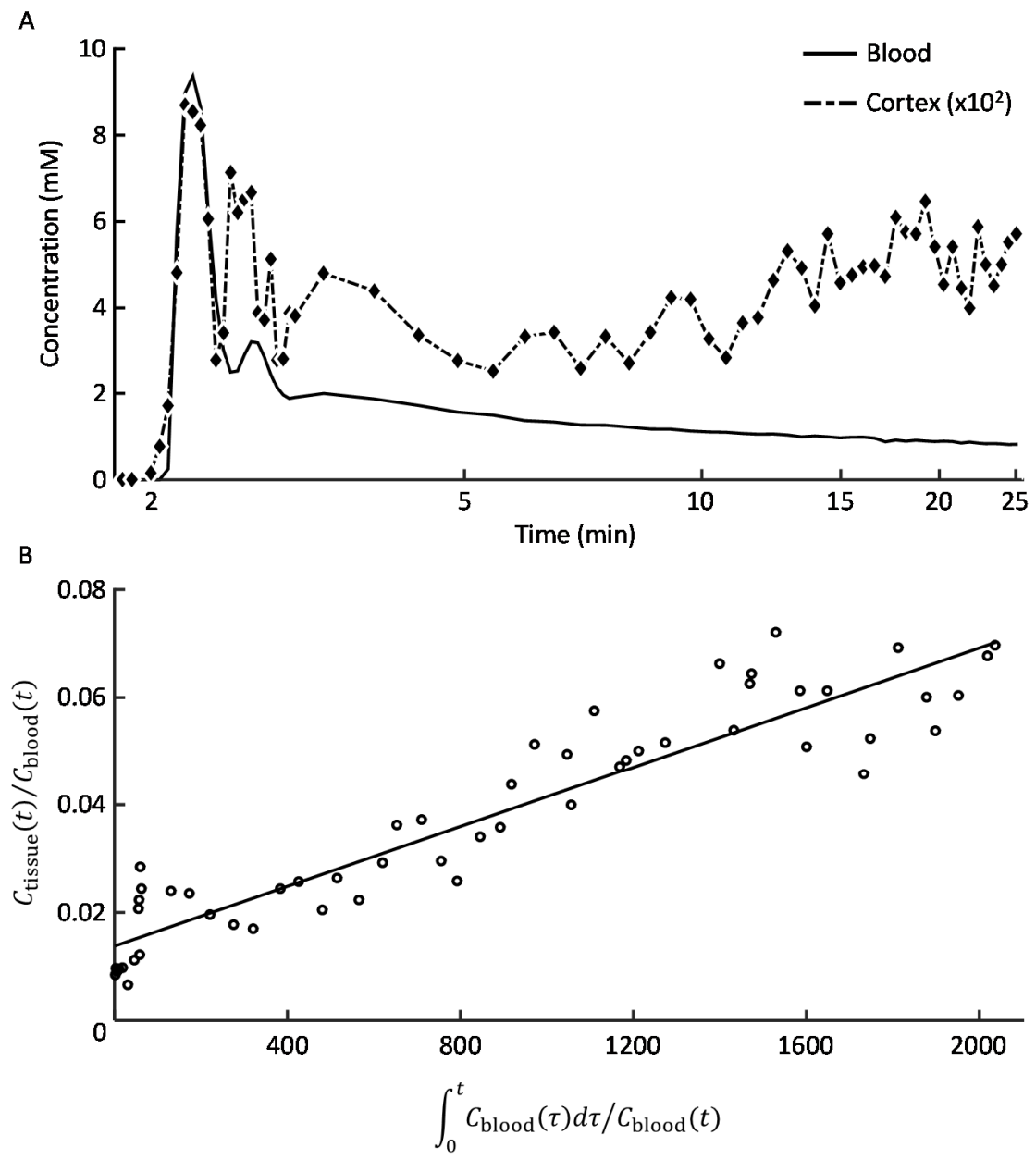

Figure 5.2: Example concentration-time curves (A) and a Patlak plot (B) of a patient. The vascular input function and a concentration curve of a single cortex voxel exhibiting relatively strong leakage are shown $(A)$. Note that the time scale is logarithmic to better show the initial arrival of the contrast agent. The corresponding Patlak plot (B) results in a $v_{p}$ of $1.4 \%$ and $a K_{i}$ of $1.6 \times 10^{-3} \mathrm{~min}^{-1}$. 

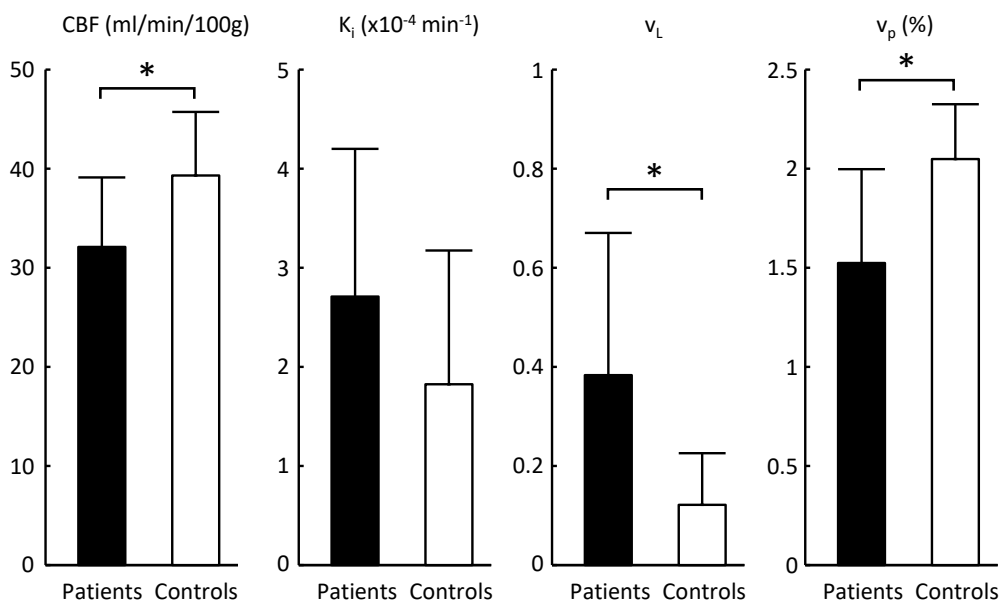

Figure 5.3: The differences in cerebral blood flow (CBF), blood-brain barrier leakage rate $\left(\mathrm{K}_{\mathrm{i}}\right)$, fraction of leaking gray matter voxels $\left(\mathrm{V}_{\mathrm{L}}\right)$, and local blood plasma volume $\left(\mathrm{V}_{\mathrm{p}}\right)$ of the total gray matter between the patients with early $A D$ and controls. The error bars denote the standard deviation. *: $p<0.05$.

Table 5.1: Cerebral blood flow per brain region

\begin{tabular}{lllll} 
Brain region & $\begin{array}{l}\text { Patients } \\
(\mathrm{mean} \pm \mathrm{s.d} . \\
\mathrm{ml} / 100 \mathrm{~g} / \mathrm{min})\end{array}$ & $\begin{array}{l}\text { Controls } \\
(\mathrm{mean} \pm \mathrm{s.d} . \\
\mathrm{ml} / 100 \mathrm{~g} / \mathrm{min})\end{array}$ & $\begin{array}{l}\text { Difference } \\
\text { relative to } \\
\text { controls }(\%)\end{array}$ & $\mathrm{p}^{\#}$ \\
\hline Total cortex & $32.8 \pm 7.2$ & $40.2 \pm 6.7$ & -22.6 & $<0.001^{*}$ \\
Frontal cortex & $33.3 \pm 7.6$ & $38.9 \pm 7.2$ & -16.8 & $0.009^{*}$ \\
Parietal cortex & $32.4 \pm 7.4$ & $39.6 \pm 6.7$ & -22.2 & $0.001^{*}$ \\
Temporal cortex & $31.1 \pm 5.5$ & $36.8 \pm 6.2$ & -18.3 & $0.003^{*}$ \\
Occipital cortex & $30.7 \pm 9.1$ & $37.9 \pm 8.3$ & -23.5 & $0.009^{*}$ \\
$\begin{array}{l}\text { Deep gray } \\
\text { matter }\end{array}$ & $27.9 \pm 6.4$ & $33.8 \pm 5.2$ & -21.2 & $0.002^{*}$ \\
\hline
\end{tabular}

\# corrected for age and gender

* remains significant after correction for multiple comparisons 
A

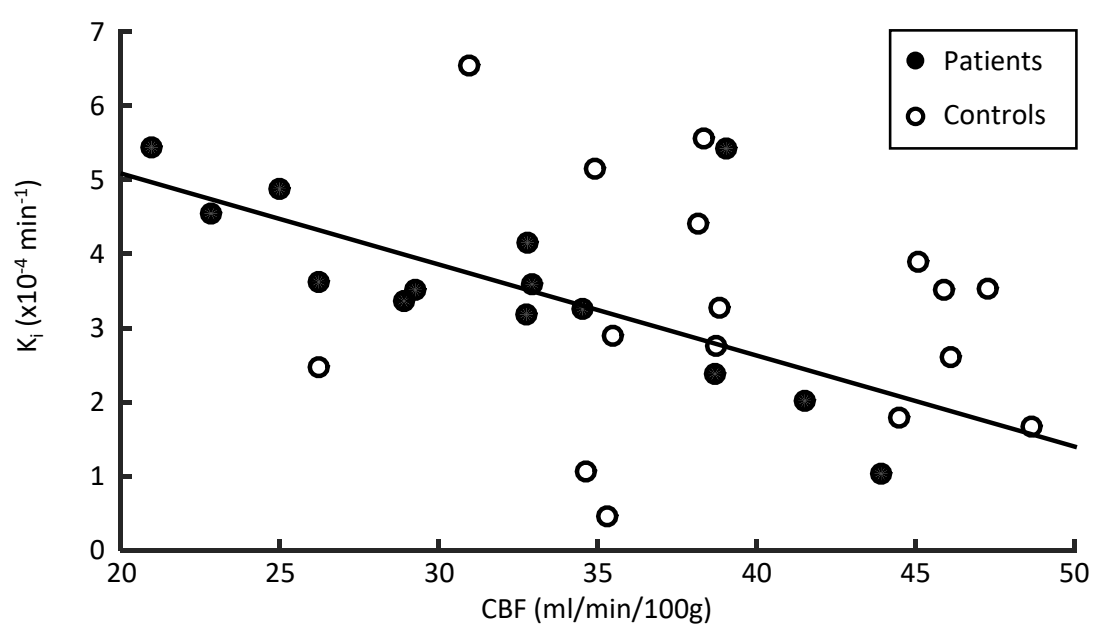

B

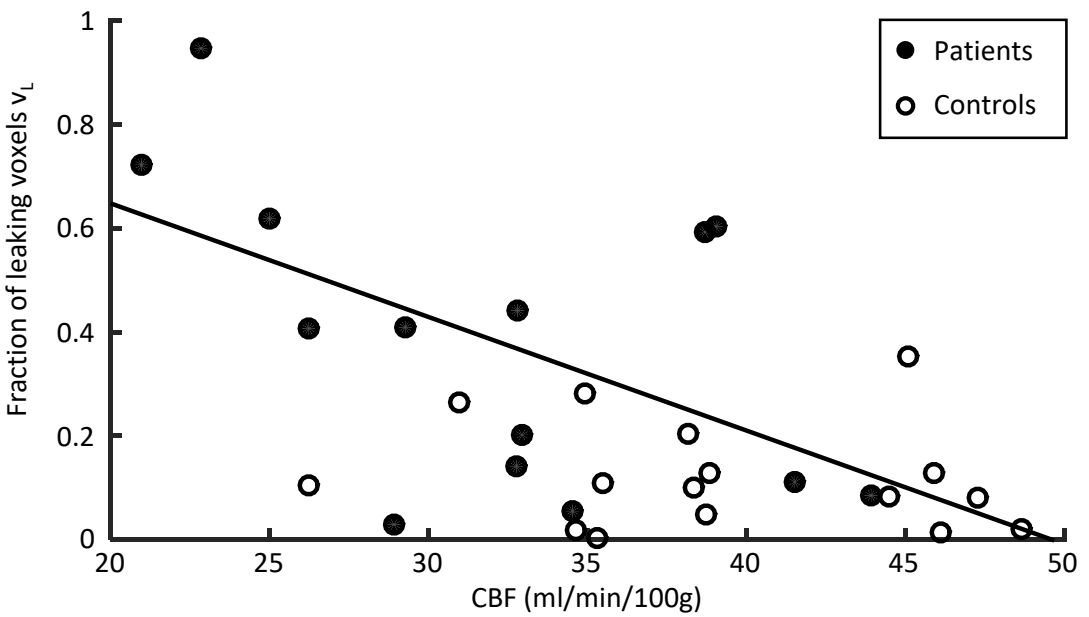

Figure 5.4: Scatter plot of local cerebral blood flow (CBF) versus blood-brain barrier leakage rate $\mathrm{K}_{\mathrm{i}}$ (A) and fraction of leaking voxels $V_{L}(B)$ of the total gray matter of the patients and controls. The line represents the (unadjusted) linear regression fit for the patients, with Pearson's $r=-0.66, p=0.006$ for $\mathrm{K}_{\mathrm{i}}$ and Pearson's $\mathrm{r}=-0.54, \mathrm{p}<0.05$ for $\mathrm{V}$. 


\section{Discussion}

In the gray matter of patients with early $A D$, we have found evidence of global hypoperfusion, a trend for a higher leakage rate and a significant increase in leakage extent and decrease of blood volume. Most interestingly, we observed that a global reduction in $\mathrm{CBF}$ is correlated with an increase in $\mathrm{BBB}$ leakage in these patients.

The cerebral blood flow values of this study (roughly $30-50 \mathrm{ml} / \mathrm{min} / 100 \mathrm{~g}$ ) are in the range of commonly reported values in healthy elderly controls and patients with $A D$

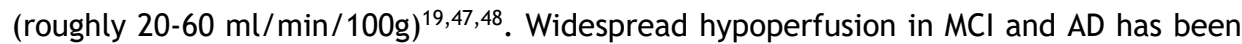
found by numerous other studies using ASL and other modalities ${ }^{49-51}$. The most common finding is hypoperfusion in the frontal and temporoparietal regions, which mostly covers the region investigated in the current study ${ }^{52}$. The relative difference in CBF between the groups is roughly the same in all lobes and in the deep gray matter (Table 5.1), indicating that the hypoperfusion is global, instead of regional, as was found in some other studies ${ }^{50,53}$. Given that the atrophy between in patients did not significantly differ from healthy controls, the observed results are unlikely to be affected by differences in cortical thickness and inherent partial volume effects of the cortex voxels. We found $K_{i}$ values of $1-3 \times 10^{-4} \mathrm{~min}^{-1}$, which are comparable to previously reported values in patients with vascular cognitive impairment $\left(0-6 \times 10^{-4} \mathrm{~min}^{-1}\right)^{54}$. The cerebral blood volume in AD has usually been studied using Dynamic Susceptibility Contrast (DSC-)MRI, where a lower blood volume is usually found in the temporoparietal regions, which are known to be impaired in $A D^{49}$. Furthermore, it is frequently mentioned that blood volume differences are smaller and harder to detect compared with blood flow ${ }^{55,56}$.

The subtle contrast agent leakage found in this study is often considered to be limited by the permeability-surface area product ${ }^{21,57,58}$. This means that, for more contrast agent to leak out of the blood space, the blood vessels have to become more permeable to the contrast agent. An alternative would be that the leakage is flow-limited, meaning that the leakage is limited by the amount of contrast agent supplied by the blood during a certain time period. For the current expected low permeability regime, the leakage should be more or less independent of the blood flow ${ }^{21,57,58}$. However, the observed correlation between CBF and BBB leakage indicates that either the leakage rate is not purely limited by the permeability-surface area product, or a common pathophysiological pathway involves both BBB breakdown and hypoperfusion. Given the very low leakage values and modest reductions in $\mathrm{CBF}$ found in this study, we consider it 
unlikely that the leakage rate is limited by the amount of contrast agent supplied by the blood. Therefore, it is more likely that a physiological process involving the neurovascular unit is responsible for this link, especially since the BBB and local compensatory mechanisms for hypoperfusion are essential functional elements of the neurovascular unit.

The link between hypoperfusion and BBB leakage extent and strength complements the previous finding that hypoperfusion is correlated with cognitive decline and hippocampal volume decrease ${ }^{59}$. Atrophy is one of the hallmarks of $A D$, but adding atrophy as a confounder did not change the (qualitative) results. Adding age as a confounder also did not change the correlation, which shows that increased BBB leakage due to age or partial volume effects caused by atrophy cannot explain the observed link between hypoperfusion and BBB leakage. Together, this all points toward a complicated progressive cascade of events that involves decreased CBF, BBB leakage and inflammation ${ }^{60}$. Although the precise pathways remain to be elucidated, the correlation between hypoperfusion and increased BBB leakage does fit to the hypothesized positive feedback loop linking the BBB and perfusion management. Such a feedback loop would cause an increase in BBB leakage when the CBF decreases and, vice versa, a decrease of $\mathrm{CBF}$ when $\mathrm{BBB}$ leakage increases. BBB leakage can be caused by ischemia triggered inflammation, while the hypoperfusion is caused by upregulation of vasoconstrictors, oxidative stress, and insufficient clearance of amyloid $B^{9}$. The recent finding that amyloid $B$ can passively pass the $B B B$ in a rat model of $A D$ further supports the neurovascular hypothesis ${ }^{61}$. Furthermore, upregulation of vasoconstrictors would by definition decrease local blood volume as was found in this study. The lower blood volume may be further explained by a lower vascular density which has also been found in $A D^{60}$.

Measuring very low BBB leakage using DCE-MRI is very challenging, and it requires a modified acquisition and analysis scheme compared with DCE-MRI in high leakage scenarios such as (high-grade) tumors ${ }^{62}$. Several studies have investigated the effect of temporal resolution, the choice of the pharmacokinetic model, signal drift, and the total scan time on the ability to detect subtle BBB leakage ${ }^{63-66}$. It is also important to use a modified acquisition sequence. For this, we decided to combine the dual-time resolution DCE-MRI protocol used by Jelescu et al. with the sequence described by Larsson et al. ${ }^{25,37}$. The dual-time resolution protocol allows for accurate sampling of the rapid signal changes during the initial arrival of the contrast agent, while the slower scan has 
a higher signal-to-noise during the slow washout, when it is more important to have a higher sensitivity to small signal changes. The sequence described by Larsson et al. allows for measurement of the T10 with slight adaptations to the scan parameters, and it also provides an analytical formula to convert the signal changes to the contrast agent concentration. However, further improvements of DCE-MRI protocols to increase sensitivity to subtle BBB leakage and decrease the influence of noise are necessary. This is especially relevant when voxelwise mapping of the leakage is desired, such as in neurodegenerative diseases where the leakage may not be localized to hotspots or clear anatomically defined regions.

In this study, the neurovascular unit was found to be impaired in patients with early AD, resulting in a lower cerebral hypoperfusion and local blood volume, and a larger fraction of leaking voxels and a trend for increased BBB leakage. This, combined with our finding that hypoperfusion and increased BBB leakage are correlated, provides further evidence for the hypothesis that vascular damage, and neurovascular impairment in particular, play a key role in the pathophysiology of $A D$.

\section{Acknowledgements}

This study was performed with support from the "Internationale Stichting Alzheimer Onderzoek" (ISAO, grant 10553). 


\section{References}

Jack CR, Knopman DS, Jagust WJ, Shaw LM, Aisen PS, Weiner MW et al. Hypothetical model of dynamic biomarkers of the Alzheimer's pathological cascade. Lancet Neurol 2010; 9: 119-128.

De La Torre JC. Is Alzheimer's disease a neurodegenerative or a vascular disorder? Data, dogma, and dialectics. Lancet Neurol 2004; 3: 184-190.

Drachman DA. The amyloid hypothesis, time to move on: Amyloid is the downstream result, not cause, of Alzheimer's disease. Alzheimer's Dement 2014; 10: 372-380.

Benarroch EE. Neurovascular unit dysfunction: A vascular component of Alzheimer disease? Neurology. 2008; 70: 1941.

Zlokovic BV. Neurovascular pathways to neurodegeneration in Alzheimer's disease and other disorders. Nat Rev Neurosci 2011; 12: 723-738.

Lecrux C, Hamel E. The neurovascular unit in brain function and disease. Acta Physiol 2011; 203: 4759.

Abbott NJ, Patabendige AAK, Dolman DEM, Yusof SR, Begley DJ. Structure and function of the bloodbrain barrier. Neurobiol. Dis. 2010; 37: 13-25.

Hawkins BT, Davis TP. The Blood-Brain Barrier / Neurovascular Unit in Health and Disease. Pharmacol Rev 2005; 57: 173-185.

ladecola C. The overlap between neurodegenerative and vascular factors in the pathogenesis of dementia. Acta Neuropathol 2010; 120: 287-296.

ladecola C. The Pathobiology of Vascular Dementia. Neuron 2013; 80: 844-866.

11 Zlokovic BV. Neurovascular mechanisms of Alzheimer's neurodegeneration. Trends Neurosci 2005; 28: 202-208.

Sagare AP, Bell RD, Zlokovic B V. Neurovascular dysfunction and faulty amyloid $\beta$-peptide clearance in Alzheimer disease. Cold Spring Harb Perspect Med 2012; 2. doi:10.1101/cshperspect.a011452.

Stanimirovic DB, Friedman A. Pathophysiology of the neurovascular unit: disease cause or consequence? J Cereb Blood Flow Metab 2012; 32: 1207-1221.

Lyros E, Bakogiannis C, Liu Y, Fassbender K. Molecular Links Between Endothelial Dysfunction and Neurodegeneration in Alzheimer's Disease. Curr Alzheimer Res 2014; 11: 18-26.

Barker R, Ashby EL, Wellington D, Barrow VM, Palmer JC, Kehoe PG et al. Pathophysiology of white matter perfusion in Alzheimer's disease and vascular dementia. Brain 2014; 137: 1524-1532.

Nicolakakis N, Hamel E. Neurovascular function in Alzheimer's disease patients and experimental models. J Cereb Blood Flow Metab 2011; 31: 1354-1370.

Farkas E, Luiten PG. Cerebral microvascular pathology in aging and Alzheimer's disease. Prog Neurobiol 2001; 64: 575-611.

Montagne A, Barnes SR, Sweeney MD, Halliday MR, Sagare AP, Zhao Z et al. Blood-Brain Barrier Breakdown in the Aging Human Hippocampus. Neuron 2015; 85: 296-302.

Alsop DC, Detre JA, Golay X, Günther M, Hendrikse J, Hernandez-Garcia L et al. Recommended implementation of arterial spin-labeled perfusion MRI for clinical applications: A consensus of the ISMRM perfusion study group and the European consortium for ASL in dementia. Magn Reson Med 2015; 73: 102-116. 
Tofts PS, Brix G, Buckley DL, Evelhoch JL, Henderson E, Knopp MV et al. Estimating kinetic parameters from dynamic contrast-enhanced T1- weighted MRI of a diffusable tracer: Standardized quantities and symbols. J Magn Reson Imaging 1999; 10: 223-232.

Dubois B, Feldman HH, Jacova C, DeKosky ST, Barberger-Gateau P, Cummings J et al. Research criteria for the diagnosis of Alzheimer's disease: revising the NINCDS-ADRDA criteria. Lancet Neurol 2007; 6: 734-746.

McKhann GM, Knopman DS, Chertkow H, Hyman BT, Jack CR, Kawas CH et al. The diagnosis of dementia due to Alzheimer's disease: Recommendations from the National Institute on AgingAlzheimer's Association workgroups on diagnostic guidelines for Alzheimer's disease. Alzheimer's Dement 2011; 7: 263-269.

Folstein MF, Folstein SE, McHugh PR. 'Mini-mental state'. A practical method for grading the cognitive state of patients for the clinician. J Psychiatr Res 1975; 12: 189-198.

Jelescu IO, Leppert IR, Narayanan S, Araújo D, Arnold DL, Pike GB. Dual-temporal resolution dynamic contrast-enhanced MRI protocol for blood-brain barrier permeability measurement in enhancing multiple sclerosis lesions. J Magn Reson Imaging 2011; 33: 1291-1300.

van de Haar HJ, Burgmans S, Jansen JFA, van Osch MJP, van Buchem MA, Muller M et al. Blood-brain barrier leakage in early Alzheimer's disease. Radiology 2016; in press.

Prins ND, Scheltens P. White matter hyperintensities, cognitive impairment and dementia: an update. Nat Rev Neurol 2015; 11: 157-165.

Jenkinson M, Bannister P, Brady M, Smith SM. Improved optimisation for the robust and accurate linear registration and motion correction of brain images. Neuroimage 2002; 17: 825-841.

Van Osch MJP, Teeuwisse WM, Van Walderveen M a a, Hendrikse J, Kies D a., Van Buchem M a. Can arterial spin labeling detect white matter perfusion signal? Magn Reson Med 2009; 62: 165-173.

Herscovitch P, Raichle ME. What Is the Correct Value for the Brain Blood Partition-Coefficient for Water. J Cereb Blood Flow Metab 1985; 5: 65-69.

Lu H, Clingman C, Golay X, Van Zijl PCM. Determining the longitudinal relaxation time (T1) of blood at 3.0 tesla. Magn Reson Med 2004; 52: 679-682.

Haroon HA, Buckley DL, Patankar TA, Dow GR, Rutherford SA, Balériaux D et al. A Comparison of Ktrans Measurements Obtained with Conventional and First Pass Pharmacokinetic Models in Human Gliomas. J Magn Reson Imaging 2004; 19: 527-536.

Li KL, Zhu XP, Waterton J, Jackson a. Improved 3D quantitative mapping of blood volume and endothelial permeability in brain tumors. J Magn Reson Imaging 2000; 12: 347-357.

Lavini C, Verhoeff JJC. Reproducibility of the gadolinium concentration measurements and of the fitting parameters of the vascular input function in the superior sagittal sinus in a patient population. Magn Reson Imaging 2010; 28: 1420-1430.

Schabel MC, Parker DL. Uncertainty and bias in contrast concentration measurements using spoiled gradient echo pulse sequences. Phys Med Biol 2008; 53: 2345-2373.

Pintaske J, Martirosian P, Graf H, Erb G, Lodemann K-P, Claussen CD et al. Relaxivity of Gadopentetate Dimeglumine (Magnevist), Gadobutrol (Gadovist), and Gadobenate Dimeglumine (MultiHance) in human blood plasma at 0.2, 1.5, and 3 Tesla. Invest Radiol 2006; 41: 213-221. and blood-brain barrier permeability, using dynamic contrast-enhanced T1-weighted MRI at 3 tesla. Magn Reson Med 2009; 62: 1270-1281.

Patlak CS, Blasberg RG, Fenstermacher JD. Graphical evaluation of blood-to-brain transfer constants 
from multiple-time uptake data. J Cereb Blood Flow Metab 1983; 3: 1-7.

Fischl B, Salat DH, Busa E, Albert M, Dieterich M, Haselgrove C et al. Whole brain segmentation: Automated labeling of neuroanatomical structures in the human brain. Neuron 2002; 33: 341-355.

Moroney JT, Bagiella E, Desmond DW, Hachinski VC, Mölsä PK, Gustafson L et al. Meta-analysis of the Hachinski Ischemic Score in pathologically verified dementias. Neurology 1997; 49: 1096-1105.

Hughes CP, Berg L, Danziger WL, Coben LA, Martin RL. A new clinical scale for the staging of dementia. Br J Psychiatry 1982; 140: 566-572.

Morris JC. The Clinical Dementia Rating (CDR): Current version and scoring rules. Neurology 1993; 43: 2412-2412.

Scheltens P, Leys D, Barkhof F, Huglo D, Weinstein HC, Vermersch P et al. Atrophy of medial temporal lobes on MRI in 'probable' Alzheimer's disease and normal ageing: diagnostic value and neuropsychological correlates. J Neurol Neurosurg Psychiatry 1992; 55: 967-972.

Fazekas F, Kleinert R, Offenbacher H, Schmidt R, Kleinert G, Payer F et al. Pathologic correlates of incidental MRI white matter signal hyperintensities. Neurology 1993; 43: 1683-1689.

Wechsler D. WAIS-III/WMS-III Technical Manual Update, 3rd ed. Psychological Corporation: San Antonio, 1997.

van der Elst W, van Boxtel MPJ, van Breukelen GJP, Jolles J. The Letter Digit Substitution Test: normative data for 1,858 healthy participants aged 24-81 from the Maastricht Aging Study (MAAS): influence of age, education, and sex. J Clin Exp Neuropsychol 2006; 28: 998-1009.

47

Binnewijzend MA, Kuijer JPA, Van Der Flier WM, Benedictus MR, Möller CM, Pijnenburg YAL et al. Distinct perfusion patterns in Alzheimer's disease, frontotemporal dementia and dementia with Lewy bodies. Eur Radiol 2014; 24: 2326-2333.

Dai W, Lopez OL, Carmichael OT, Becker JT, Kuller LH, Gach HM. Mild cognitive impairment and alzheimer disease: patterns of altered cerebral blood flow at MR imaging. Radiology 2009; 250: 856866.

Chen W, Song X, Beyea S, Arcy RD, Zhang Y. Advances in perfusion magnetic resonance imaging in Alzheimer's disease. Alzheimer's Dement 2011; 7: 185-196.

Alsop DC, Dai W, Grossman M, Detre JA. Arterial spin labeling blood flow MRI: Its role in the early characterization of Alzheimer's disease. J. Alzheimer's Dis. 2010; 20: 871-880.

Austin BP. Effects of Hypoperfusion in Alzheimer's Disease. J Alzheimers Dis 2011; 26: 123-133.

Kehoe EG, McNulty JP, Mullins PG, Bokde ALW. Advances in MRI biomarkers for the diagnosis of Alzheimer's disease. Biomark Med 2014; 8: 1151-69.

Mattsson N, Tosun D, Insel PS, Simonson A, Jack CR, Beckett LA et al. Association of brain amyloid- $\beta$ with cerebral perfusion and structure in Alzheimer's disease and mild cognitive impairment. Brain 2014; 137: 1550-1561.

4

Taheri S, Gasparovic C, Shah NJ, Rosenberg G a. Quantitative measurement of blood-brain barrier permeability in human using dynamic contrast-enhanced MRI with fast T1 mapping. Magn Reson Med 2011; 65: 1036-1042.

Lacalle-Aurioles M, Mateos-Pérez JM, Guzmán-De-Villoria J a, Olazarán J, Cruz-Orduña I, AlemánGómez $Y$ et al. Cerebral blood flow is an earlier indicator of perfusion abnormalities than cerebral blood volume in Alzheimer's disease. J Cereb Blood Flow Metab 2014; : 654-659.

6 Uh J, Lewis-Amezcua K, Martin-Cook K, Cheng Y, Weiner M, Diaz-Arrastia R et al. Cerebral blood volume in Alzheimer's disease and correlation with tissue structural integrity. Neurobiol Aging 2010; 
31: $2038-2046$.

57 Sourbron SP, Buckley DL. Classic models for dynamic contrast-enhanced MRI. NMR Biomed 2013; 26: 1004-1027.

58 Larsson HBW, Tofts PS. Measurement of blood-brain barrier permeability using dynamic Gd-DTPA scanning-a comparison of methods. Magn Reson Med 1992; 24: 174-176.

59 Roher A, Debbins, Malek-Ahmadi M, Pipe, Maze, Belden et al. Cerebral blood flow in Alzheimer's disease. Vasc Health Risk Manag 2012; 42: 599.

60 Janota C, Lemere C a., Brito MA. Dissecting the Contribution of Vascular Alterations and Aging to Alzheimer's Disease. Mol Neurobiol 2015. doi:10.1007/s12035-015-9319-7.

Keaney J, Walsh DM, O'Malley T, Hudson N, Crosbie DE, Loftus T et al. Autoregulated paracellular clearance of amyloid- across the blood-brain barrier. Sci Adv 2015; 1: e1500472-e1500472.

Armitage PA, Farrall AJ, Carpenter TK, Doubal FN, Wardlaw JM. Use of dynamic contrast-enhanced MRI to measure subtle blood-brain barrier abnormalities. Magn Reson Imaging 2011; 29: 305-314.

Cramer SP, Larsson HBW. Accurate determination of blood-brain barrier permeability using dynamic contrast-enhanced T1-weighted MRI: a simulation and in vivo study on healthy subjects and multiple sclerosis patients. J Cereb Blood Flow Metab 2014; 34: 1655-65.

Heye AK, Thrippleton MJ, Armitage PA, Valdés Hernández M del C, Makin SD, Glatz A et al. Tracer kinetic modelling for DCE-MRI quantification of subtle blood-brain barrier permeability. Neuroimage 2016; 125: 446-455.

Barnes SR, Ng TSC, Montagne A, Law M, Zlokovic BV., Jacobs RE. Optimal acquisition and modeling parameters for accurate assessment of low $\mathrm{K}$ trans blood-brain barrier permeability using dynamic contrast-enhanced MRI. Magn Reson Med 2016; 75: 1967-1977.

Cramer SP, Simonsen H, Frederiksen JL, Rostrup E, Larsson HBW. Abnormal blood-brain barrier permeability in normal appearing white matter in multiple sclerosis investigated by MRI. Neurolmage Clin 2014; 4: 182-189. 


\section{Chapter 6}

\section{The effect of white matter}

hyperintensities on

\section{hippocampal structural connectivity}

in Alzheimer's disease

Harm J van de Haar, Jacobus FA Jansen, Heidi IL Jacobs, Saartje Burgmans, Ed HBM Gronenschild, Ron LH Handels, Pauline Aalten, Lies Clerx, Inez Ramakers, Frans RJ Verhey, Walter H Backes 


\section{Abstract}

White matter hyperintensities (WMHs) are commonly found in Alzheimer's disease (AD), and are presumed to represent effects due to microvascular pathology. Previous studies indicate that WMHs affect the white matter connections. The aim of this study was to investigate the association between WMH load and the volume of the hippocampal tracts. Using diffusion MRI combined with tractography, the tracts connecting the hippocampi with the cerebrum were reconstructed in 26 participants with subjective memory complaints, 20 with mild cognitive impairment, and 14 with AD. The tractspecific WMH load and relative tract volume of the hippocampal tracts were calculated. The results show that tract-specific WMH load increases with relative tract volume. A possible explanation for this observation may be that WMHs cause pathological hypertrophy of the white matter tracts, or that the white matter fibers reposition near the WMHs. This work further elucidates the effect of WMHs on the white matter tracts and on cognition. 


\section{Introduction}

Although traditionally not regarded a manifest neuropathological sign of Alzheimer's disease $(A D)$, many patients diagnosed with $A D$ exhibit vascular pathology ${ }^{1}$. Signs of such vascular pathology are hyperintensities in the white matter (WMHs) on brain MR images ${ }^{2}$. While their precise etiology is still unclear, WMHs are assumed to be an indication of cerebrovascular pathology ${ }^{3,4}$. WMHs are considered a normal part of aging, but severe and confluent WMHs are associated with cognitive decline $e^{2,4,5}$. Besides vascular pathology, WMHs also show demyelination and axonal loss ${ }^{6}$. Therefore, WMHs may negatively affect white matter bundles intersecting them. This effect of the WMHs would likely influence the entire white matter tract rather than merely causing local changes (also referred to as diaschisis), and therefore also influence the effectiveness of information transfer throughout the brain. For example, it has been found that a higher diffusivity of streamlines passing through WMHs in dementia is associated with stronger cognitive decline ${ }^{7}$. In multiple sclerosis, it was found that a single localized lesion causes altered diffusivity markers along the entire bundle, and the local damage may ultimately cause degeneration of the entire neuronal bundle ${ }^{8,9}$. There is also evidence that WMHs cause a decoupling of the structural connectivity of white matter tracts and the functional connectivity of the regions connected by these tracts ${ }^{10}$. Overall, it seems likely that WMHs are associated with disruption of the white matter tracts, which ultimately leads to cognitive decline.

Structural brain connectivity can be measured using diffusion MRI (dMRI), combined with tractography to visualize the white matter tracts. In the presence of white matter fibers, the diffusion of water becomes more directional in the direction of the white matter fibers (anisotropic) rather than random (isotropic), which can be measured using dMRI ${ }^{11}$. Application of $d M R I$ in $A D$ has shown widespread damage to the white matter, which is expressed through a decreased fractional anisotropy and increased mean diffusivity mainly in the frontal and temporal white matter, which is already detectable at a very early stage ${ }^{12}$. Using tractography, the three-dimensional space taken up by the white matter fibers can be estimated ${ }^{13-15}$. The reconstructed white matter fiber pathways give an indication of the architectural connectivity patterns of the brain.

In the current study, we primarily set out to investigate the effect of the white matter tracts when they cross WMHs. The study was performed in older adults evaluated for cognitive impairment. The hippocampal region is known to be affected already before 
cognitive symptoms in $A D^{16}$. In addition, various white matter tracts connecting the hippocampus with other parts of the brain, such as the cingulum and uncinate fasciculus, have been found to exhibit decreased fractional anisotropy in $A D^{17}$. In this study, we focused on the white matter tracts connecting the hippocampi with the rest of the cerebrum. We used dMRI to reconstruct the pathways of the structural connections between the hippocampus and the rest of the brain in participants who were referred to the memory clinic and diagnosed with subjective memory complaints (SMC), mild cognitive impairment $(\mathrm{MCl})$ or with dementia due to AD. Secondarily, we also studied the possible effect of WMHs in the hippocampal tracts on episodic memory, processing speed and executive functioning.

\section{Materials and Methods}

\section{Participants}

The participants represent the first-time visitors at the memory clinic of the Maastricht University Medical Centre (MUMC+), the Netherlands, who were referred due to of cognitive complaints. All participants were selected from the Maastricht data of the Leiden-Alzheimer Research Netherlands (LeARN) consortium, a multicenter study on novel biomarkers for $A D$. Further information on participant inclusion and exclusion criteria, is described in a previously published paper ${ }^{18}$. Ultimately, from a total of 76 , we included 60 participants (10 were excluded for missing data and 6 for failure of the preprocessing pipeline caused by imaging artifacts). Of these 60 participants, 26 were diagnosed with subjective memory complaints (SMC), 20 with mild cognitive impairment $(\mathrm{MCl})$ and 14 with mild dementia due to $A D$. The diagnoses of $\mathrm{MCl}$ due to $A D$ and dementia due to $A D$ were made by experts at the memory clinic, based on the core clinical criteria of the National Institute on Aging-Alzheimer's Association workgroups on diagnostic guidelines for Alzheimer's disease ${ }^{19,20}$.

\section{Medical background}

All participants were interviewed regarding their medical history including (cerebro)vascular pathology and underwent standard neurological and psychiatric assessments, and neuropsychological tests, including the Mini-Mental State Evaluation (MMSE) for global cognition ${ }^{21}$, 15-word Verbal Learning Test (VLT) (immediate recall and delayed recall for episodic memory $)^{22,23}$, and the Stroop Color-Word test for processing speed (part 1) as well as executive functioning (part 3) ${ }^{24}$. Based on structural MRI scans, 
the visually rated medial temporal lobe atrophy score was determined ${ }^{25}$. Written informed consent was obtained from all participants, and the study was approved by the Medical Ethical Committee of MUMC+.

\section{MRI acquisition}

All imaging was performed on a 3T MRI system (Philips Achieva, Best, the Netherlands) with an 8-channel head coil. Diffusion weighted imaging was performed using a highangular resolution diffusion imaging (HARDI) sequence with 61 directions and a b-value of $1000 \mathrm{~s} / \mathrm{mm}^{2}$, one $\mathrm{b}=0 \mathrm{scan}, \mathrm{TR} / \mathrm{TE} 8250 / 80 \mathrm{~ms}, 128 \times 128$ matrix, 70 slices and a $2 \times 2 \times 2$ $\mathrm{mm}^{3}$ voxel size. A T1-weighted structural scan (TR/TE $8.2 / 3.7 \mathrm{~ms}, 8^{\circ}$ flip angle, $240 \times 240$ matrix, 180 slices and a $1 \times 1 \times 1 \mathrm{~mm}^{3}$ voxel size) was used for anatomical reference, and a fluid-attenuated inversion recovery sequence (FLAIR, TR/TE/TI 11000/125/2800 ms, $512 \times 512$ matrix, 48 slices and a $0.5 \times 0.5 \times 3 \mathrm{~mm}^{3}$ voxel size) was used for $\mathrm{WMH}$ assessment (further details follow in the next paragraphs).

\section{MRI analysis}

The dMRI data were preprocessed using ExploreDTI (www.exploredti.com, v4.8.326). First, the diffusion weighted images were corrected for head motion and eddy current induced distortions. Next, using the T1-weighted image as reference, corrections were performed for echo-planar imaging distortions ${ }^{27}$. After the corrections, whole-brain deterministic tractography was performed based on constrained spherical deconvolution (CSD) reconstruction of the fiber pathways. The CSD tractography parameters included uniformly distributed seeds with a seed-point resolution of $0.5 \times 0.5 \times 0.5 \mathrm{~mm}^{3}$, a fiber orientation distribution threshold of 0.1 , a maximum harmonic degree of 8 , a maximum fiber deflection angle of $30^{\circ}$ and a minimum fiber length of $50 \mathrm{~mm}$. This resulted in $\sim 2.5$ million tracts per dataset (range 1.9 - 3.1 million). The diffusion tensor was estimated per voxel to calculate the mean diffusivity $(M D)$ and the fractional anisotropy (FA).

The T1-weighted structural scans were segmented using the FreeSurfer software package (www.freesurfer.net, version 5.1.0, manually corrected where necessary ${ }^{28}$ ), which yielded 82 cortical and subcortical gray matter regions of interest (including both hippocampi), and also provided intracranial, gray matter and white matter volumes. To obtain a measure of global atrophy, an atrophy score was calculated using (gray matter volume + white matter volume) / intracranial volume ${ }^{29}$. The FLAIR images were used to semi-automatically detect the WMHs, based on a method described by De Boer et al. ${ }^{30}$. 
All WMHs were manually checked and corrected where necessary. A global WMH load was calculated by dividing the WMH volume by the total white matter volume.

Further analysis was performed in Matlab (version 2012b, MathWorks, Natick, MA, USA $)^{31}$. The tractography data were used to find white matter tracts connecting the hippocampi with the remaining 80 cortical and subcortical gray matter regions and the tracts connecting the left with the right hippocampus. A connection was defined if a streamline passed through both regions. In cases where streamlines did not start or end within the regions of interest, only the section of the streamline connecting the regions was considered. The coordinates of all streamlines connecting the hippocampus with the region-of-interest were used to calculate the total volume of the space covered by the connections. A voxel was included if a minimum of three connecting streamlines crossed the voxel, to avoid false positives ${ }^{32}$. The volume of the tract which overlapped with WMHs was also determined. The white matter tract volume and the tract-specific WMH volume of the hippocampal tracts were normalized to the total white matter volume as obtained with FreeSurfer. This resulted in the relative tract volume and the relative tract-specific WMH load, respectively.

\section{Statistical analysis}

All statistical analyses were performed using Matlab. The group characteristics were compared using independent-samples (two-sided) Student's t-tests for continuous variables and chi-squared tests for dichotomous variables (i.e. sex and the incidence of (cerebro)vascular pathology). The scores of the neuropsychological tests were compared between the groups using linear regression analyses, corrected for age, sex and years of education.

The difference between the groups in mean volume of the white matter tracts connecting the hippocampi with the rest of the cerebrum was first tested using multivariate linear regression. The mean MD and FA of this tract volume were also compared between the groups using regression analysis. Next, the relations between white matter tract volume, tract specific WMH load and cognition were calculated. Three different cognitive domains were tested: episodic memory measured by the VLT delayed word recall (VLTDR) 22,23 , processing speed measured by the Stroop color-word test part 1 (Stroop 1) and executive function measured by the Stroop $3^{24}$. A hierarchical method was employed: first relations between the relevant variables spanning the entire population were calculated, and if significant correlations were found, the participants 
were divided into the three groups, based on the clinical diagnosis. To correct for age, sex, the incidence of hypertension, and years of education, these characteristics were included as covariates in all regression models. To account for potential group differences, the MMSE score was also added as a covariate when testing over all participants, as a proxy for the diagnosis. A false discovery rate (FDR) procedure $(q=0.1)$ was applied to correct for multiple comparisons of the groups, brain regions or cognitive tests, when relevant ${ }^{33}$. All regression is performed using a robust least-squares method to account for outliers, all reported B's are standardized, and statistical significance was inferred when $\mathrm{p}<0.05$.

\section{Results}

\section{Participant characteristics}

The group characteristics are listed in Table 6.1. The participants with AD exhibited a significantly higher global WMH load and medial temporal lobe atrophy score compared with the SMC participants, and significantly higher global atrophy compared with both the SMC and $\mathrm{MCl}$ participants. There was also a significant difference in sex between the $S M C$ and $A D$ groups, and the $A D$ patients were significantly older than the SMC and $M C I$ patients, which was adjusted for in the subsequent regression analysis.

An overview of the performance on the neuropsychological tests per group is provided in Table 6.2. The immediate recall part of the VLT showed significant differences between all three groups, while the Stroop 1 score did not show significant differences, and the other neuropsychological tests showed significant differences between some, but not all, of the groups. 
Table 6.1: Group characteristics

\begin{tabular}{llll} 
& SMC & MCI & AD \\
\hline Number of participants & 26 & 20 & 14 \\
Sex (\% female) & 31 & 45 & $64^{*}$ \\
Age (y) & $61.7(12.0)$ & $67.9(8.2)$ & $78.0(6.7)^{*} \dagger$ \\
Education (y) & $11.0(3.4)$ & $10.1(2.7)$ & $10.3(3.7)$ \\
Total cerebral tissue volume $\left(\mathrm{mlx} 10^{3}\right)$ & $1.07(0.07)$ & $1.07(0.11)$ & $0.96(0.08)$ \\
White matter volume $\left(\mathrm{mlx} 10^{3}\right)$ & $0.47(0.05)$ & $0.47(0.07)$ & $0.43(0.04)$ \\
Gray matter volume $\left(\mathrm{mlx} 10^{3}\right)$ & $0.60(0.04)$ & $0.60(0.05)$ & $0.53(0.04)$ \\
Atrophy score (\%) & $68.4(4.2)$ & $67.6(4.1)$ & $63.4(2.8)^{*} \dagger$ \\
MTA score & $1.3(1.5)$ & $2.3(1.7)$ & $3.2(1.4)^{*}$ \\
Global WMH load (\%) & $2.4(3.6)$ & $3.1(3.0)$ & $5.7(5.2)^{*} \dagger$ \\
Number of microbleeds & $1.3(2.4)$ & $0.5(0.7)$ & $2.1(5.1)$ \\
Number of lacunes & $0.7(1.1)$ & $0.2(0.6)$ & $0.5(1.1)$ \\
Hypertension (\%) & 19 & 15 & 21 \\
Cerebrovascular pathology (\%) & 19 & $0 \ddagger$ & 7 \\
Cardiovascular pathology (\%) & 27 & 35 & 36 \\
\hline
\end{tabular}

SMC: Subjective Memory Complaints, MCI: Mild Cognitive Impairment, AD: Alzheimer's Disease, WMH: White Matter Hyperintensities, MTA: Medial Temporal lobe Atrophy ${ }^{25}$.

Cerebrovascular pathology includes a history of stroke, aneurysm, or evidence of traumatic brain injury. Cardiovascular pathology includes hypertension, coronary artery disease, and cardiac pathology. The values between parentheses are standard deviations. All statistical tests were independent two-sided t-tests, except for sex and the presence of cerebrovascular and cardiovascular pathology, which were tested using a chi-squared test. Global WMH load was log-transformed prior to statistical testing because of the skewed distribution. *: significant difference between SMC and $A D, \ddagger$ : significant difference between $S M C$ and $M C l, \dagger$ : significant difference between $M C l$ and $A D$. Significance was inferred at $p<0.05$. 


\section{Effect of WMH on tract volume}

An example of the hippocampal tracts is displayed in Figure 6.1. The absolute tract volumes, relative tract volumes and the tract WMH loads are listed in Table 6.3. The regression analysis did not show a significant difference in the mean volume of tracts connecting the hippocampus with the rest of the gray matter between any of the groups $(p>0.2)$. There was a significant positive association between tract volume and the mean relative WMH load over all participants $(B=0.86, p<0.001$, also see Figure 6.2$)$. Repeating the same analysis in the three groups showed that this association was mainly present in the $A D$ patients $(B=1.01, p<0.001)$ and in the $S M C$ group $(B=0.51, p=0.035$, both of which remained significant after FDR correction over the groups), but not in the $\mathrm{MCI}$ patients $(p=0.10)$. Neither the mean MD $(p>0.11)$ or FA $(p>0.07)$ in the hippocampal tracts was significantly different between any of the groups after FDR correction over the groups.

Table 6.2: Neuropsychological test scores

\begin{tabular}{lllll}
$\begin{array}{l}\text { Neuropsychological test } \\
\text { (score range) }\end{array}$ & $\begin{array}{l}\text { Cognitive } \\
\text { domain }\end{array}$ & SMC & MCI & AD \\
\hline MMSE (0-30) & $\begin{array}{l}\text { Global } \\
\text { cognition }\end{array}$ & $28.8(1.4)$ & $28.1(2.2)$ & $24.5(2.6)^{*} \dagger$ \\
VLT, immediate recall (0-75) & $\begin{array}{l}\text { Working } \\
\text { memory }\end{array}$ & $42.0(9.9)$ & $30.2(6.8) \ddagger$ & $20.4(5.8)^{*} \dagger$ \\
VLT, delayed recall (0-15) & $\begin{array}{l}\text { Episodic } \\
\text { memory }\end{array}$ & $8.9(3.3)$ & $3.6(2.6) \neq$ & $2.2(4.1)^{*}$ \\
Stroop 1 (in sec) & $\begin{array}{l}\text { Processing } \\
\text { speed }\end{array}$ & $51.3(8.8)$ & $54.9(12.6)$ & $65.7(20.3)$ \\
Stroop 3 (in sec) & $\begin{array}{l}\text { Executive } \\
\text { function }\end{array}$ & $112.0(34.6)$ & $149.0(46.7)$ & $187.4(71.2)^{*}$ \\
\hline
\end{tabular}

MMSE: Mini-Mental State Evaluation, VLT: Verbal Learning Test.

The values between parentheses are standard deviations. All statistical tests were corrected for age, 


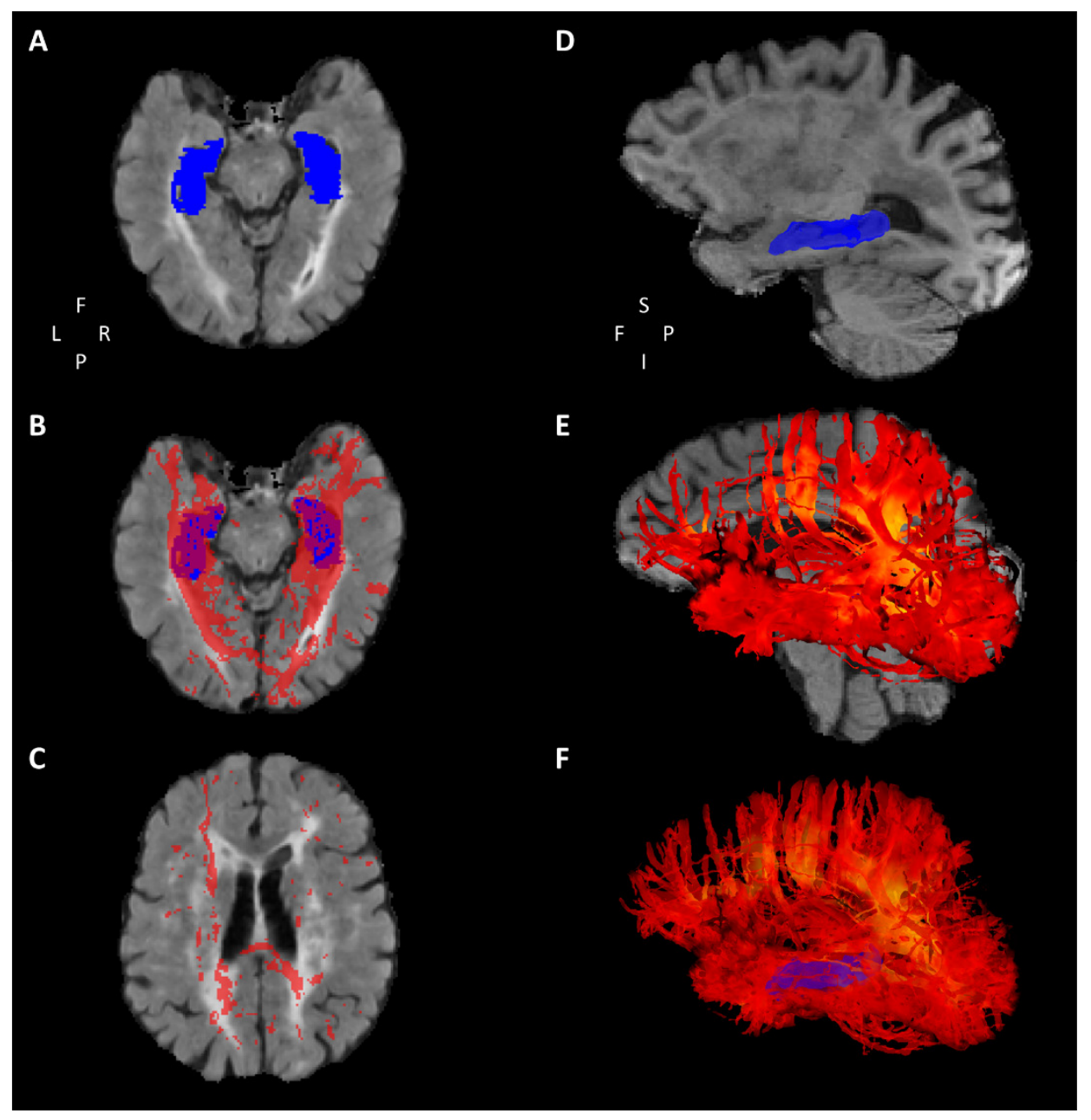

Figure 6.1: An overview of the hippocampal tracts of a single patient diagnosed with Alzheimer's disease. In A, the FLAIR is shown with the hippocampi colored blue. The tracts are displayed as a red overlay on the same slice (B), and at a higher slice (C), which is above the hippocampi so they are not visible. In D, the T1 structural image with a blue 3D render of the left hippocampus is shown. The tracts of the left hemisphere are shown as a 3D render in E, with the T1 slice located just beyond the interhemispheric fissure. The tract volume is colored orange to yellow, scaled by the intensity of the FLAIR image. Therefore, areas colored yellow correspond to areas where the hippocampal tracts pass through white matter hyperintensities. In $F$, the $3 D$ render of the tracts is shown for both hemispheres. Note how the tracts in B, C, E and F overlap the white matter hyperintensities, indicating that white matter fibers pass through these areas. 
Table 6.3: Volumetry of hippocampal connections

\begin{tabular}{|c|c|c|c|c|}
\hline & Region connected with hippocampus & SMC & $\mathrm{MCl}$ & $A D$ \\
\hline \multirow{5}{*}{$\begin{array}{l}\text { Tract } \\
\text { volume } \\
\text { (ml) }\end{array}$} & Whole cerebral cortex & $0.90(0.23)$ & $0.82(0.14)$ & $0.82(0.26)$ \\
\hline & Frontal & $0.39(0.18)$ & $0.29(0.11)$ & $0.31(0.17)$ \\
\hline & Temporal & $1.12(0.26)$ & $1.03(0.22)$ & $1.04(0.27)$ \\
\hline & Parietal & $0.84(0.27)$ & $0.93(0.35)$ & $0.71(0.29)$ \\
\hline & Occipital & $1.28(0.54)$ & $1.29(0.56)$ & $1.30(0.84)$ \\
\hline \multirow{5}{*}{$\begin{array}{l}\text { Relative } \\
\text { tract } \\
\text { volume } \\
(\%)\end{array}$} & Whole cerebral cortex & $1.94(0.52)$ & $1.76(0.31)$ & $1.91(0.62)$ \\
\hline & Frontal & $1.41(0.42)$ & $1.31(0.37)$ & $1.48(0.65)$ \\
\hline & Temporal & $1.71(0.51)$ & $1.64(0.31)$ & $1.56(0.55)$ \\
\hline & Parietal & $2.44(0.67)$ & $2.17(0.57)$ & $2.31(0.82)$ \\
\hline & Occipital & $1.43(0.41)$ & $1.40(0.39)$ & $1.44(0.44)$ \\
\hline \multirow{5}{*}{$\begin{array}{l}\text { Tract WMH } \\
\text { load (\%) }\end{array}$} & Whole cerebral cortex & $0.32(0.25)$ & $0.39(0.36)$ & $0.48(0.33)$ \\
\hline & Frontal & $0.16(0.13)$ & $0.22(0.17)$ & $0.34(0.28)$ \\
\hline & Temporal & $0.25(0.26)$ & $0.29(0.22)$ & $0.30(0.30)$ \\
\hline & Parietal & $0.30(0.32)$ & $0.42(0.56)$ & $0.50(0.50)$ \\
\hline & Occipital & $0.29(0.27)$ & $0.52(0.42)$ & $0.54(0.39)$ \\
\hline $\begin{array}{l}\text { Fractional } \\
\text { anisotropy }\end{array}$ & Whole cerebral cortex & $0.29(0.04)$ & $0.30(0.03)$ & $0.29(0.04)$ \\
\hline $\begin{array}{l}\text { Mean } \\
\text { diffusivity } \\
\left(\times 10^{-3}\right. \\
\left.\mathrm{mm}^{2} / \mathrm{s}\right)\end{array}$ & Whole cerebral cortex & $1.24(0.13)$ & $1.21(0.11)$ & $1.28(0.09)$ \\
\hline
\end{tabular}

SMC: Subjective Memory Complaints, MCl: Mild Cognitive Impairment, AD: Alzheimer's Disease, WMH: White Matter Hyperintensities

The values between parentheses are standard deviations. All statistical tests were corrected for age, gender and years of education. The tract volume represents the mean volume of the individual tracts between the hippocampus and cortical regions. The raw tract volume was not statistically tested. The relative tract volume and the relative WMH load were normalized to the total white matter volume. No statistically significant differences were found. 


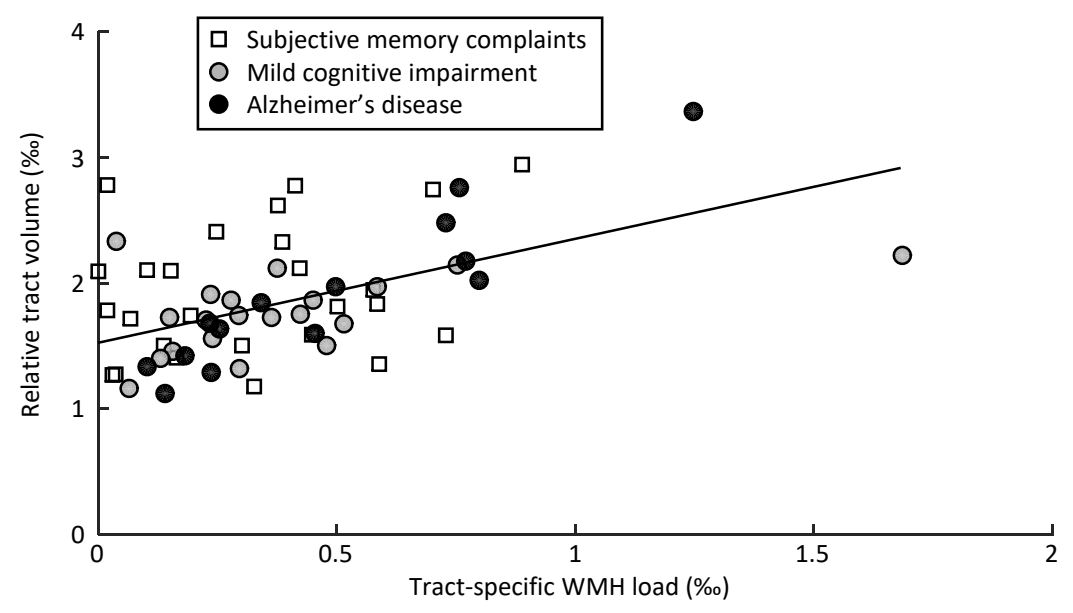

Figure 6.2: The relative hippocampal tract volume versus the tract-specific white matter hyperintensity (WMH) load for the different groups. The displayed fit is based on the raw (not corrected for confounders) data of all the participants for visualization purposes. The fit reported in the manuscript is based on a robust method and corrected for age, gender, the incidence of hypertension, and years of education, and it has a standardized $\beta$ of 0.86 and a p-value smaller than 0.001 .

Post hoc, we also investigated whether there was an association between the volume of the tracts connecting the hippocampi with the various cerebral lobes and the corresponding tract-specific WMH load. The association between relative tract volume and tract-specific WMH load appeared strongest for the fronto-hippocampal connections $(B=0.80, p<0.001)$ and parieto-hippocampal connections $(B=0.64, p<0.001)$, and was also significant for the temporo-hippocampal (intratemporal) connections $(B=0.42, p=0.003$ ), all of which remained significant after correction for multiple comparisons over the four lobes. There was a trend for an association between the relative tract volume of the occipito-hippocampal connections and the corresponding WMH load $(B=0.27, p=0.07$, which remains a trend after FDR correction over the lobes). Also, note that the occipitohippocampal tracts were the largest tracts (Table 6.3).

\section{Effect of tract-specific WMH on cognition}

None of the cognitive domains showed an association with either relative tract volume or tract-specific WMH load $(p>0.1)$. However, because relative tract volume and tractspecific WMH load were correlated, we reasoned that these measures may also reinforce 
one another and together have an effect on cognition. Further investigation revealed that the interaction between increased relative tract volume and tract-specific WMH load was associated with a decreased processing speed $(B=1.98, p<0.001)$ and with a decreased executive function $(B=1.44, p=0.003)$, both of which remained significant after FDR correction over the three cognitive domains. The episodic memory did not show an association with the interaction between increased relative tract volume and tract-specific WMH load $(p=0.4)$. Repeating this analysis for the different groups for both the processing speed and executive function scores revealed that this association mainly presented in the $A D$ patients $(p=0.041$ for processing speed and a trend with $p=0.055$ for executive function), although the association was weak. There were no associations for either cognitive domain in the SMC or $\mathrm{MCl}$ groups $(\mathrm{p}>0.2)$.

\section{Discussion}

\section{Main findings}

The aim of the current study was to investigate the effect of WMHs on the volume of the white matter tracts connecting the hippocampi with the rest of the cerebral gray matter. The study showed a correlation between increased relative white matter tract volume and tract WMH load, which was strongest for the fronto-hippocampal connections. No direct associations between cognition and relative tract volume or tract-specific WMH load were found. However, decreases in both processing speed and executive function were found to be associated with the interaction between an increased relative tract volume and an increase in tract-specific WMH load.

\section{Association tract volume and WMH load}

The results showed that a higher tract-specific WMH load was associated with a higher relative tract volume. When interpreting this result, it is important to note that the patients with $A D$ had a significantly lower global atrophy score and higher medial temporal lobe atrophy score compared with the SMC group. This indicates that structural brain changes were already present in the $A D$ group, which is to be expected as cerebral atrophy is one of the hallmarks of $A D$. Therefore, when interpreting the physiological meaning behind the correlation between a higher tract-specific WMH load and larger white matter tracts, it is likely that the white matter tracts are not enlarged, but that they do not atrophy as fast as other white matter tracts or cortical tissue. This would cause the bundles to remain relatively large compared with the cerebral tissue volume. 
The results displayed in Table 6.3 seem to corroborate this, because they show that the absolute volume of the white matter tracts becomes smaller in the AD patients, while the relative tract volume stays roughly the same, although the differences are not significant.

It is also important to note that a significant correlation between a higher tract volume and a higher tract WMH load may be caused by the larger tracts having an increased chance of incorporating WMHs. However, the results were strongest in the frontohippocampal connections, which also happen to be the smallest. On the other hand, the occipito-hippocampal connections, which are the largest tracts, did not show this correlation. Therefore, larger tracts also incorporating more WMHs by chance is unlikely the explanation for the observed correlation between tract volume and tract WMH load. Other explanations may be that WMHs cause pathological hypertrophy of the white matter tracts, or that the demyelination of the neurons in the WMHs causes the white matter bundles to be rerouted in and around the WMHs. It was previously found that a higher WMH load was associated with cortical thickening (hypertrophy) ${ }^{34}$, hypothetically due to inflammation. This could also explain how the WMHs affect the tracts, as inflammation and glial cell activation have been found in both $A D$ and WMHs specifically ${ }^{35}$. Both the hypertrophy and rerouting explanations assume that WMHs impose an active effect on the white matter tracts. Such structural effects of WMHs would probably be expressed in MD and FA differences, which were not shown by our diffusion metrics. This was surprising, as an increased MD and decreased FA in WMHs are widely reported in the literature ${ }^{36-38}$. The absence of significant differences in diffusion metrics in the current study may be due to the relatively early stage of the disease process of the patients. Future studies will have to validate these findings. Furthermore, the correlations were strongest in the fronto-, parieto-, and temporo-hippocampal connections, which also correspond to the widely reported progression of structural changes occurring over the transition from health to $A D^{39}$. Our results indicated that the correlation between tract-specific WMH load and tract volume was only significant in the $A D$ group, which implies that in early stages of the disease, the white matter tracts are still largely unaffected, even though WMHs may be present.

\section{Association of cognition with interaction tract volume and $\mathrm{WMH}$ load}

The results showed that neither the severity of WMH load on the hippocampal tracts nor the volume of the tracts was significantly associated with cognitive function. However, 
both processing speed and executive function decreased significantly with the interaction between tract-specific WMH load and tract volume. An interpretation of this result is that, although these cognitive measures and the tract measures are not directly correlated, when tracts are relatively large with a relatively high WMH load, they are associated with cognitive decline. The finding that this was strongest in the AD patients indicates that this effect mostly occurs when the cognitive decline has progressed to such an extent that daily living activities are impacted and the WMH load is the largest. The main cognitive domains associated with WMHs are psychomotor speed and executive function, although a wide range of domains have been reported to be affected by $\mathrm{WMHs}^{35,40-42}$. However, despite the fact that the correlation between WMH load and cognitive decline is widely reported, the association can be hard to detect ${ }^{7,43,44}$. A possible explanation for this is the pathological heterogeneity of $A D$, but the current results offer another possible explanation. The interaction between tract volume and WMH load shows that WMHs may only affect cognition if they cause white matter tract alterations, indicating that not all WMHs are detrimental to the connectivity network. This may be because the WMHs are not at critical locations, or possibly because they are not yet large enough to influence function (cognition). This is supported by the findings that not all WMHs affect cognition, and that the location of the WMHs rather than the extent determines the impact on the cognitive function ${ }^{45,46}$. The process through which WMHs affect cognition is still unclear. It has been hypothesized that disruption of white matter may cause cognitive decline through impairing signal transmission ${ }^{47}$. The WMH related demyelination may be the prime cause of this signal transmission impairment, which might prompt compensatory mechanisms such as rerouting or expansion of the white matter tracts. The results of this study provide further evidence that WMHs affect the structure of the white matter tracts, which eventually influences cognition.

\section{Clinical relevance}

WMHs in $A D$ may modify $A D$ pathology, increasing cognitive decline and the risk of dementia ${ }^{35,48}$. The results of this study are in concordance with this hypothesis because the effect of WMHs on cognition is strongest in the $A D$ group and thus seems tied to $A D$ pathology. Furthermore, WMHs are strongly associated with vascular pathology, and, given their interactive role in cognitive decline and $A D$ pathology, may be a sign of vascular problems exacerbating the symptoms ${ }^{4,37,44}$. Therefore, the current results stress the importance of preventing and treating vascular risk factors in the elderly. The 
presence of WMHs indicates vascular pathology and may be interpreted as a warning sign.

\section{Study considerations}

The main strength of this study is that the methodology introduced in this study may be utilized in different brain regions and connections. Applying the connection-specific method to other diseases with possible white matter pathology involvement may reveal new pathological pathways and connections, because it allows for a much more specific and targeted assessment. A limitation of this study is that only three cognitive domains were investigated. Another limitation is that the current patient population was based on first-time visitors of a memory clinic. This means that the patients in the current study were in relatively early stages of $A D$, which is reflected in the relatively high MMSE scores. Possible correlations between tract volume and cognition may only be detectable at later stages of the disease, which has to be further investigated in future studies.

\section{Summary and conclusion}

To summarize, in participants experiencing cognitive decline, white matter tracts connecting the hippocampi with the rest of the brain are relatively larger in size if they incorporate WMHs, illustrating that WMHs affect white matter connectivity. The methodology that was presented in the current study can be applied in other neurodegenerative diseases to find specific white matter connections that are affected by pathological processes.

\section{Acknowledgements}

We would like to thank Swantje Schade for the assessment and correction of the WMH segmentations. This research was performed within the framework of the CTMM, The Center for Translational Molecular Medicine (www.ctmm.nl), project LeARN (grant 02N-101). 


\section{References}

Echávarri C, Burgmans S, Caballero MC, García-Bragado F, Verhey FRJ, Uylings HBM. Co-occurrence of different pathologies in dementia: Implications for dementia diagnosis. J Alzheimer's Dis 2012; 30: 909-917.

Burns JM, Church J a, Johnson DK, Xiong C, Marcus D, Fotenos AF et al. White matter lesions are prevalent but differentially related with cognition in aging and early Alzheimer disease. Arch Neurol 2005; 62: 1870-1876.

Gouw AA, Seewann A, Vrenken H, Van Der Flier WM, Rozemuller JM, Barkhof F et al. Heterogeneity of white matter hyperintensities in Alzheimer's disease: Post-mortem quantitative MRI and neuropathology. Brain 2008; 131: 3286-3298.

Prins ND, Scheltens P. White matter hyperintensities, cognitive impairment and dementia: an update. Nat Rev Neurol 2015; 11: 157-165.

Tomimoto $\mathrm{H}$. White matter integrity and cognitive dysfunction: Radiological and neuropsychological correlations. Geriatr Gerontol Int 2015; 15: 3-9.

Gouw AA, Seewann A, van der Flier WM, Barkhof F, Rozemuller AM, Scheltens P et al. Heterogeneity of small vessel disease: a systematic review of MRI and histopathology correlations. J Neurol Neurosurg Psychiatry 2011; 82: 126-135.

Reginold W, Luedke AC, Tam A, Itorralba J, Fernandez-Ruiz J, Reginold J et al. Cognitive Function and 3-Tesla Magnetic Resonance Imaging Tractography of White Matter Hyperintensities in Elderly Persons. Dement Geriatr Cogn Dis Extra 2015; 5: 387-394.

Droby A, Fleischer V, Carnini M, Zimmermann H, Siffrin V, Gawehn J et al. The impact of isolated lesions on white-matter fiber tracts in multiple sclerosis patients. Neurolmage Clin 2015; 8: 110116.

Siffrin V, Vogt J, Radbruch H, Nitsch R, Zipp F. Multiple sclerosis - candidate mechanisms underlying CNS atrophy. Trends Neurosci 2010; 33: 202-210.

Reijmer YD, Schultz AP, Leemans A, O'Sullivan MJ, Gurol ME, Sperling R et al. Decoupling of structural and functional brain connectivity in older adults with white matter hyperintensities. Neuroimage 2015; 117: 222-9.

Basser PJ, Jones DK. Diffusion-tensor MRI: Theory, experimental design and data analysis - A technical review. NMR Biomed 2002; 15: 456-467.

Amlien IK, Fjell AM. Diffusion tensor imaging of white matter degeneration in Alzheimer's disease and mild cognitive impairment. Neuroscience 2014; 276: 206-215.

Jones DK. Determining and visualizing uncertainty in estimates of fiber orientation from diffusion tensor MRI. Magn Reson Med 2003; 49: 7-12.

Basser PJ, Pajevic S, Pierpaoli C, Duda J, Aldroubi A. In vivo fiber tractography using DT-MRI data. Magn Reson Med 2000; 44: 625-632.

Mori S, Crain BJ, Chacko VP, Van Zijl PCM. Three-dimensional tracking of axonal projections in the brain by magnetic resonance imaging. Ann Neurol 1999; 45: 265-269.

Scahill RI, Schott JM, Stevens JM, Rossor MN, Fox NC. Mapping the evolution of regional atrophy in Alzheimer's disease: unbiased analysis of fluid-registered serial MRI. Proc Natl Acad Sci U S A 2002; 99: 4703-7. Networks in Alzheimer's Disease: A Diffusion MRI Study with DTI and HARDI Models. Neural Plast 
2016; 2016: 1-14.

Handels RL, Aalten P, Wolfs CA, OldeRikkert M, Scheltens P, Visser P et al. Diagnostic and economic evaluation of new biomarkers for Alzheimer's disease: the research protocol of a prospective cohort study. BMC Neurol 2012; 12: 72.

Albert MS, DeKosky ST, Dickson D, Dubois B, Feldman HH, Fox NC et al. The diagnosis of mild cognitive impairment due to Alzheimer's disease: Recommendations from the National Institute on Aging and Alzheimer's Association workgroup. Alzheimer's Dement 2011; 7: 270-279.

McKhann GM, Knopman DS, Chertkow H, Hyman BT, Jack CR, Kawas $\mathrm{CH}$ et al. The diagnosis of dementia due to Alzheimer's disease: Recommendations from the National Institute on AgingAlzheimer's Association workgroups on diagnostic guidelines for Alzheimer's disease. Alzheimer's Dement 2011; 7: 263-269.

Folstein MF, Folstein SE, McHugh PR. 'Mini-mental state'. A practical method for grading the cognitive state of patients for the clinician. J Psychiatr Res 1975; 12: 189-198.

Brand N, Jolles J. Learning and Retrieval Rate of Words Presented Auditorily and Visually. J Gen Psychol 1985; 112: 201-210.

Rey A. L'examen clinique en psychologie. Presse Universitaires de France: Paris, 1964.

Stroop JR. Studies of interference in serial verbal reactions. J Exp Psychol 1935; 18: 643-662.

Scheltens P, Leys D, Barkhof F, Huglo D, Weinstein HC, Vermersch P et al. Atrophy of medial temporal lobes on MRI in 'probable' Alzheimer's disease and normal ageing: diagnostic value and neuropsychological correlates. J Neurol Neurosurg Psychiatry 1992; 55: 967-972.

Leemans A., Jeurissen B, Sijbers J, Jones D. ExploreDTI: a graphical toolbox for processing, analyzing, and visualizing diffusion MR data. Proc 17th Sci Meet Int Soc Magn Reson Med 2009; 17: 3537.

Irfanoglu MO, Walker L, Sarlls J, Marenco S, Pierpaoli C. Effects of image distortions originating from susceptibility variations and concomitant fields on diffusion MRI tractography results. Neuroimage 2012; 61: 275-288.

Fischl B, Salat DH, Busa E, Albert M, Dieterich M, Haselgrove C et al. Whole brain segmentation: Automated labeling of neuroanatomical structures in the human brain. Neuron 2002; 33: 341-355.

Buckner RL, Head D, Parker J, Fotenos AF, Marcus D, Morris JC et al. A unified approach for morphometric and functional data analysis in young, old, and demented adults using automated atlas-based head size normalization: Reliability and validation against manual measurement of total intracranial volume. Neuroimage 2004; 23: 724-738.

de Boer R, Vrooman HA, van der Lijn F, Vernooij MW, Ikram MA, van der Lugt A et al. White matter lesion extension to automatic brain tissue segmentation on MRI. Neuroimage 2009; 45: 1151-1161.

van Bussel FCG, Backes WH, Hofman PAM, van Boxtel MPJ, Schram MT, Stehouwer CDA et al. Altered hippocampal white matter connectivity in Type 2 Diabetes Mellitus and memory decrements. $J$ Neuroendocrinol 2016; : n/a-n/a.

Vaessen MJ, Jansen JF a, Vlooswijk MCG, Hofman P a M, Majoie HJM, Aldenkamp AP et al. White matter network abnormalities are associated with cognitive decline in chronic epilepsy. Cereb Cortex 2012; 22: 2139-2147.

Benjamini Y, Hochberg Y. Controlling the False Discovery Rate: a Practical and Powerful Approach to Multiple Testing. J R Stat Soc 1995; 57: 289-300.

Jacobs HIL, Clerx L, Gronenschild EHBM, Aalten P, Verhey FRJ. White matter hyperintensities are positively associated with cortical thickness in Alzheimer's disease. J Alzheimers Dis 2014; 39: 40922. 
Wardlaw JM, Valdés Hernández MC, Muñoz-Maniega S. What are white matter hyperintensities made of? Relevance to vascular cognitive impairment. J Am Heart Assoc 2015; 4: 001140.

Bastin ME, Clayden JD, Pattie A, Gerrish IF, Wardlaw JM, Deary IJ. Diffusion tensor and magnetization transfer MRI measurements of periventricular white matter hyperintensities in old age. Neurobiol Aging 2009; 30: 125-136.

Maniega SM, Valdés Hernández MC, Clayden JD, Royle NA, Murray C, Morris Z et al. White matter hyperintensities and normal-appearing white matter integrity in the aging brain. Neurobiol Aging 2015; 36: 909-918. MRI of Corpus Callosum Tracts Crossing White Matter Hyperintensities. AJNR Am J Neuroradiol 2016; : 1-6.

Serra L, Cercignani M, Lenzi D, Perri R, Fadda L, Caltagirone C et al. Grey and white matter changes at different stages of Alzheimer's disease. J Alzheimers Dis 2010; 19: 147-159.

Te M, Zhao E, Xingyue Z, Qinjian S, Chuanqiang Q. Leukoaraiosis with mild cognitive impairment. Neurol Res 2015; 37: 410-414.

Frisoni GB, Galluzzi S, Pantoni L, Filippi M. The effect of white matter lesions on cognition in the elderly--small but detectable. Nat Clin Pract Neurol 2007; 3: 620-627.

Van Der Vlies AE, Staekenborg SS, Admiraal-Behloul F, Prins ND, Barkhof F, Vrenken $\mathrm{H}$ et al. Associations between magnetic resonance imaging measures and neuropsychological impairment in early and late onset alzheimer's disease. J Alzheimer's Dis 2013; 35: 169-178.

Ku BD, Na DL, Moon SY, Kim SY, Seo SW, Cheong HK et al. Neuropsychological correlates of the proportional impact of white matter hyperintensities on mild to moderate dementia: The MRI 300 study. Dement Geriatr Cogn Disord 2011; 31: 397-405.

Shim Y, Morris J, Cairns N, Benzinger T, Xiong C. Pathological correlates of white matter hyperintensities on magnetic resonance imaging. Alzheimer's Dement 2011; 7: S706.

Smith EE, Salat DH, Jeng J, McCreary CR, Fischl B, Schmahmann JD et al. Correlations between MRI white matter lesion location and executive function and episodic memory. Neurology 2011; 76 : 1492-1499.

Jacobs HIL, Visser PJ, Van Boxtel MPJ, Frisoni GB, Tsolaki M, Papapostolou P et al. The association between white matter hyperintensities and executive decline in mild cognitive impairment is network dependent. Neurobiol Aging 2012; 33: 201.e1-8. compromise prefrontal cortex function in healthy elderly individuals. J Cogn Neurosci 2006; 18: 41829.

Debette S, Markus HS. The clinical importance of white matter hyperintensities on brain magnetic resonance imaging: systematic review and meta-analysis. BMJ 2010; 341: c3666. 



\section{Chapter 7}

General discussion 


\section{Thesis overview}

The aim of the research presented in this thesis was to investigate the extent and nature of microvascular damage in Alzheimer's disease. To this end, multiple Magnetic Resonance Imaging (MRI) sequences were used to investigate different forms and effects of neurovascular pathology in patients suffering from Alzheimer's disease. This was studied both in the earliest phases of the disease, as well as in full-blown Alzheimer's disease. Specifically, we used Dynamic Contrast-Enhanced (DCE) MRI to investigate leakage of the blood-brain barrier, Arterial Spin Labeling (ASL) to measure local cerebral blood flow, and diffusion MRI to assess the effect of white matter hyperintensities (WMHs) of presumed vascular origin on the white matter bundles connecting the hippocampi with other brain regions. The combination of these methods resulted in a novel evaluation of damage to the cerebral microvessels and the subsequent effects on the white matter in Alzheimer's disease.

\section{Main findings}

The blood-brain barrier is part of the neurovascular unit, which is a collection of cells and subcellular structures incorporated in the wall of the cerebral microvessels, along with other supporting cells, which together protect and support the neurons ${ }^{1-3}$. Vascular pathology has been implicated as being an important part of the pathophysiology of Alzheimer's disease, and the blood-brain barrier is a prime suspect ${ }^{4}$. Therefore, the first questions of this thesis were: "In what ways can the integrity of the blood-brain barrier be non-invasively measured in vivo?" and subsequently "What techniques have already been used to measure blood-brain barrier integrity in early Alzheimer's disease in vivo?". In Chapter 2, an extensive literature search revealed that different methods can be used to measure blood-brain barrier integrity, and that these techniques have been applied in different diseases causing dementia, and at different stages of these diseases. A commonly used method is measuring the amount of albumin in the cerebrospinal fluid relative to the albumin concentration in the blood ${ }^{5}$. Since albumin can enter the cerebrospinal fluid by passing the blood-brain barrier, a process which is strictly regularized, a disproportionate amount of albumin in the cerebrospinal fluid indicates some failure of this regularization ${ }^{6}$. However, even though the albumin ratio may indicate blood-brain barrier impairment, it does not provide any further information on the spatial extent or location of this pathology. Neuroimaging techniques based on the use of contrast agents offer an alternative method to evaluate the blood-brain barrier in vivo. Chapter 2 shows that the first investigation of the blood-brain barrier in patients 
with dementia using neuroimaging was performed with Positron Emission Tomography (PET) in combination with a gallium-68 tracer. Later, Computed Tomography (CT) and MRI were introduced and applied to study the blood-brain barrier in dementia. Different contrast media allowed for the investigation of both active and passive transport mechanisms of the blood-brain barrier. Overall, MRI in combination with a gadolinium based contrast agent seems to be the most popular neuroimaging method for detection of blood-brain barrier impairment. However, actual damage to the blood-brain barrier in a non-vascular form of dementia was not reported at that time, even though postmortem and albumin ratio studies did show that the blood-brain barrier is likely damaged or dysfunctional in Alzheimer's disease.

This prompted the next research question: "Is there blood-brain barrier impairment in early Alzheimer's disease?”. To investigate this, an MRI study, using novel insights and a dedicated DCE-MRI protocol, was applied in patients with early Alzheimer's disease and healthy controls. The protocol and results of this research are discussed in Chapter 3. A dual-time resolution DCE-MRI protocol, in combination with the Patlak graphical approach to separate the blood signal from signal of extravasating contrast agent, was chosen to examine blood-brain barrier leakage in patients with early Alzheimer's disease. A noise suppression method and novel measure for leakage extent (rather than leakage rate) were developed to increase sensitivity. The result was that there is indeed (passive) leakage of contrast agent though the blood-brain barrier in early Alzheimer's disease, although it is very subtle and hard to detect. The in vivo data also showed that the blood-brain barrier leakage is proportional with cognitive decline, which suggests that the leakage worsens as the disease progresses.

Even with the inclusion of the noise suppression method, the subtle blood-brain barrier leakage was still very difficult to measure, which might complicate further investigation of the effects of blood-brain barrier impairment in neurodegenerative diseases. Therefore, the next research question was: "How can the sensitivity of a DCE-MRI experiment be improved to detect subtle blood-brain barrier leakage?". To assist future studies and gain further insight into the effect of noise and methodological differences, a simulation study was performed, which is described in Chapter 4. The simulations revealed that DCE-MRI, which is commonly used to investigate high-grade tumors ${ }^{7}$, overestimates the degree of blood-brain barrier leakage in neurodegenerative diseases, and that the measured leakage rate depends on the total scan time and probably also on the specific MRI sequence. Besides the scan time-dependent bias, it has also been 
shown that such low leakage rates are below the detection limit on the single voxel level, which shows that measuring the absolute voxelwise leakage rate may not be feasible ${ }^{8}$. However, the results also showed that the noise suppression method is feasible, and that comparisons of the leakage rate between groups can be made with proper statistical power.

Besides the blood-brain barrier, other functional elements of the neurovascular unit may also be impaired in Alzheimer's disease ${ }^{4,9-11}$. Damage to the microvessels will likely have an effect on the distribution of blood throughout the brain. This prompted the next question: “Is the local blood perfusion in early Alzheimer's disease altered?". The local blood flow can be measured using MRI with an endogenous contrast agent by utilizing $\mathrm{ASL}^{12}$. In Chapter 5, the details and results of applying ASL in patients with early Alzheimer's disease is described. It was revealed that already in early stages of Alzheimer's disease, patients have global hypoperfusion in the gray matter, indicating that not only the blood-brain barrier may be impaired, but also the local regulation of blood flow. The next question therefore was: “Is there a correlation in early Alzheimer's disease between blood flow impairment and other functional elements of the neurovascular unit?". In Chapter 5, the results of the DCE-MRI experiment were combined with the ASL data to evaluate the relation between local blood flow and the blood-brain barrier impairment. This revealed that, indeed, hypoperfusion is correlated with an increase of the blood-brain barrier leakage rate. Together, the blood-brain barrier leakage and hypoperfusion indicate that different functional elements of the neurovascular unit are impaired and, because they are related, it is likely that Alzheimer's disease affects the neurovascular unit as a whole.

The microvascular effects of Alzheimer's disease also manifest themselves in other ways. A very common finding in the elderly is the presence of white matter hyperintensities, which are presumed to be ischemic in nature and thus reflect microvascular pathology ${ }^{13,14}$. The previous chapters have shown that microvascular damage is widespread in Alzheimer's disease, and that this damage may be linked to cognitive function. However, the pathway through which vascular damage may influence cognitive decline is unknown. One possible hypothesis is that WMHs are markers of local vascular and neuronal impairment, that influence the white matter connections of the brain $^{13,15}$. Therefore, the next question was: "What is the impact of WMHs on the brain white matter in terms of structural connectivity?". Using diffusion MRI, the white matter tracts of participants experiencing different degrees of cognitive decline and 
dementia were visualized. It was found that the WMHs are associated with a relative increase in the volume of the white matter bundles which connect the hippocampi with the rest of the brain. This may indicate either a compensatory effect through the rerouting of the white matter fibers, or a swelling of the bundles caused by the microvascular damage.

\section{Conceptual background}

The brain is responsible for roughly $20 \%$ of the total body oxygen consumption ${ }^{16}$. Given that the brain only comprises $2 \%$ of the human body weight, the brain uses the most energy of all organs. The neurons rely on the neurovascular unit to supply all that energy, while protecting them from neurotoxic substances circulating in the blood. The neurovascular unit is a collection of cells and subcellular structures in the cerebral microvascular wall ${ }^{1}$. These elements cooperate to regulate local cerebral blood flow, the passage of molecules through the vascular wall and generally support the functioning of the neurons.

The classical signs of Alzheimer's disease are the accumulation of insoluble proteins and the atrophy of the cortex of the temporal lobe ${ }^{17}$. There are, however, other signs pointing towards a much broader underlying pathophysiology involving a global energy crisis. One commonly reported sign is hypometabolism found using FDG-PET ${ }^{18}$. Another sign is the reduced cerebral blood flow as we also demonstrated using arterial spin labeling in Chapter 5. This energy crisis could cause dysfunctioning of the neurons, which leads to cognitive impairment and dementia ${ }^{19,20}$.

Unfortunately, the pathophysiology of Alzheimer's disease is still not understood completely. It is clear that accumulation of the protein amyloid $B$ is at least associated with Alzheimer's disease, but exactly how is still unknown ${ }^{21}$. The most important missing piece of the puzzle is the pathway through which amyloid $B$ damages neurons ${ }^{22}$. It also remains unclear what the temporal relationship is between amyloid $B$ aggregation and the cognitive decline, and how other factors, most notably vascular pathology, fit into this hypothesized pathological cascade ${ }^{23}$.

The involvement of the blood-brain barrier in the pathophysiology of Alzheimer's disease is very likely. The blood-brain barrier is a complicated structure which has many different pathways regulating transport of molecules ${ }^{2}$. Many of these pathways have already been shown to be impaired in Alzheimer's disease ${ }^{24}$. In Chapter 3, we have shown that contrast agents leak faster through the blood-brain barrier in patients with 
Alzheimer's disease than in control subjects. This allows for the possibility that also other, undesirable substances can exit the blood pool and could enter the surrounding tissue. This could lead to initiation of inflammation processes, leading to further neuronal damage ${ }^{25}$. The added value of the research described in this research is that it provides in vivo proof for blood-brain barrier leakage in patients with an early stage of Alzheimer's disease. Moreover, it provides information on the localization of this leakage. Instead of hotspots at anatomically identifiable regions, such as were found in the white matter hyperintensities in vascular cognitive impairment ${ }^{26,27}$, the leakage was found to be diffuse and widespread. This widespread leakage fits the global nature of many signs of Alzheimer's disease. For example, the atrophy of cerebral tissue, although starting at the medial temporal lobe and initially following a specific pattern, affects all brain regions in the later stages of the disease ${ }^{28}$. The plaques and tangles are also spread throughout the brain at the last stages ${ }^{29,30}$.

There is also evidence that the opening of the blood-brain barrier may actually be beneficial. It has been shown in a mouse model for Alzheimer's disease that amyloid $B$ can leak from the brain to the blood through the blood-brain barrier and influence tight junction protein levels to facilitating its own leakage, which may show a beneficial effect of opening the blood-brain barrier ${ }^{31}$. In another study using a mouse model of Alzheimer's disease, opening the blood-brain barrier using ultrasound facilitated clearance of amyloid $B$ from the brain tissue, and it also had a significant positive effect on multiple memory tasks ${ }^{32}$. These findings do have to be replicated in humans however, which raises important ethical questions on patient safety and side-effects. Because the blood-brain barrier protects the brain from harmful substances circulating in the blood, opening the blood-brain barrier may cause more harm than good. The exact effects of opening the blood-brain barrier, and whether they are beneficial or harmful, should be investigated in future studies. Furthermore, using ultrasound in the brain also introduces technical challenges due to the size of the human brain and the thickness of the skull prohibiting the use of ultrasound techniques in some brain regions ${ }^{33}$. If these challenges can be overcome in some way, the blood-brain barrier might present a new target for therapeutic intervention.

When considering the different hypotheses for Alzheimer's disease, the research presented in this thesis fits into the neurovascular hypothesis (also see Figure 7.1). According to this hypothesis, vascular risk factors initiate a complex cascade of events that leads to damage to different parts of the neurovascular unit. This cascades involves 
hypoperfusion, impaired clearance of amyloid $B$, and blood-brain barrier leakage , $^{4-11,34}$. Because amyloid $B$ causes blood-brain barrier leakage and, at the same time is also a vasoconstrictor, the damage is also self-reinforcing ${ }^{35,36}$. Therefore, accumulation of amyloid $B$ further reduces CBF and consequently causes ischemia, inflammation, oxidative stress, nitric oxide deficit, demyelination, and upregulation of vasoconstrictors ${ }^{4,20,24,25,37,38}$. In Chapter 3, evidence for widespread impairment of the blood-brain barrier is presented, while Chapter 5 shows global hypoperfusion, and the combined data shows the association between blood-brain barrier leakage, hypoperfusion and global cognition. In Chapter 6, it is shown that local changes to the microvessels, which manifest as WMHs, affects the white matter tracts, and that this is also correlated with cognition. This provides a more direct clue as to how vascular pathology may influence the cognitive function of a patient with Alzheimer's disease.

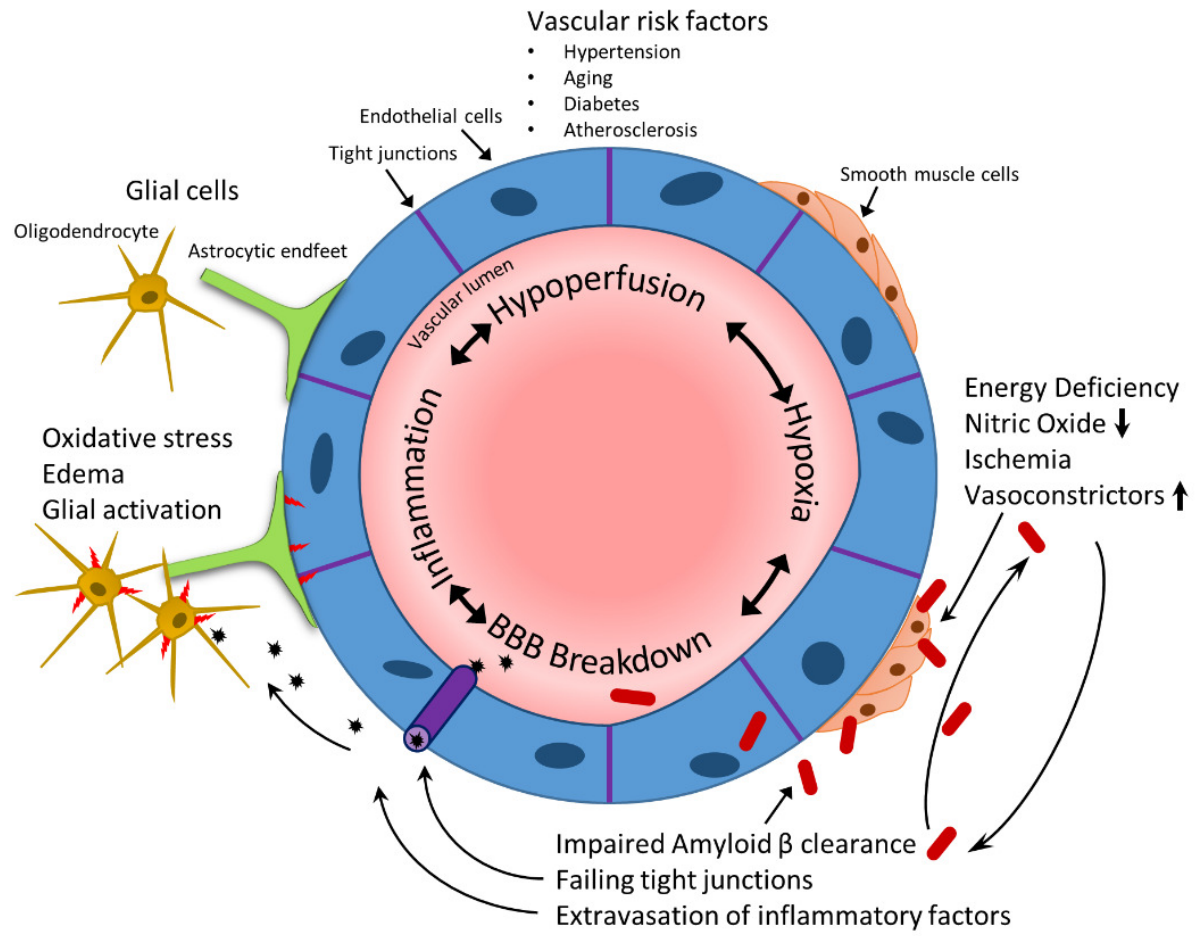

Figure 7.1: A functional biological model of the hypothesized interplay between different elements of the neurovascular unit in Alzheimer's disease. A self-reinforcing cascade of events, thought to be initiated by vascular risk factors, leads to degradation of the neurovascular unit due to increasing hypoperfusion, breakdown of the blood-brain barrier and inflammation. 


\section{Clinical implications}

The research described in this thesis reinforces that vascular pathology is associated with Alzheimer's disease. Putting this information into practice will require a paradigm shift, both for healthcare professionals and for the older adults at risk for Alzheimer's disease.

A possible way to combat widespread diseases is screening of the population. Conditions for screening include low costs, low chance for false positives and negatives and a low impact of the screening procedure for the patient. Given the impact vascular pathology has on the incidence and prognosis of Alzheimer's disease, screening the vascular health of older adults may be worth $i^{39}$. Several options are available. Simply measuring and regulating blood pressure and trying to improve overall cardiac health and condition may significantly improve the quality of life of many. This is cheap, has a relatively low burden for the patients and will also help reduce vascular disease. More extensive tests could include screening for atherosclerosis and peripheral vascular disease ${ }^{40}$.

For healthcare professionals, recognizing the importance of the vascular components of Alzheimer's disease means that treating vascular problems and conveying the importance of physical fitness becomes much more important. Besides the decreased risk of stroke or a heart attack, the decreased risk of cognitive decline and dementia are further arguments for promoting proper vascular health. It is also important to recognize that Alzheimer's disease is not a well-defined, homogeneous disease ${ }^{41}$. Many different factors may play a role in the disease process, both vascular and non-vascular, and multiple methods aiming to prevent, delay, and treat cognitive decline and dementia due to Alzheimer's disease may be needed.

For the general public, awareness of the importance of vascular health needs to be expanded. Most of the vascular risk factors for Alzheimer's disease are modifiable, meaning that the patient is able to change the likelihood of being diagnosed with Alzheimer's disease by changing their lifestyle. It is widely known that obesity and a poor physical condition increases the risk of vascular diseases. However, the association between poor vascular health and dementia is not widely known, and may persuade more people to change their unhealthy habits. To achieve this, a general awareness campaign may be very beneficial. The main goals of this awareness campaign would be to spread knowledge on the current insights into the vascular component of dementia and to provide tips on improving overall fitness and vascular health. 


\section{Strengths and limitations}

Overall, the main strength of the research in this thesis is that it shows that several promising MRI techniques have been improved to a point that they can be applied to examine different aspects of the pathophysiology of Alzheimer's disease. This includes measuring vascular function and health using ASL and DCE-MRI, blood-brain barrier integrity using DCE-MRI, and white matter bundle condition using diffusion-weighted MRI. All this information can be acquired in vivo and with a relatively low burden on the patient, using widely available MRI scanners.

The main limitations of the research presented in this thesis are that most of the findings are based on a pilot study with limited participants, and that the measured group differences are small and were hard to find. This is the consequence of performing clinical research on largely untested hypotheses. The implementation of the dualtemporal resolution DCE-MRI protocol proved to be sensitive to subtle pathology of the blood-brain barrier, but it required a very long scan time and a complicated analysis procedure. Such novel ideas first have to be tested in relatively small groups before they can be used to further investigate the pathophysiology causing cognitive decline and dementia in larger patient groups.

Improving the DCE-MRI sequence and data analysis is needed before widespread use could be advocated. This has already partly been done in Chapter 4, but the scan time of the sequence is almost half an hour, which is too long and presents quite a burden on the patient, and on the availability of the scanner. It is also sensitive to imaging artifacts, and the field-of-view of the fast segment of the dual-time resolution sequence is limited, prohibiting actual whole-brain imaging. Improvements to the hardware and further tweaking of the imaging sequence, such as optimized parallelization settings and pulse sequences, may improve image quality, time resolution, and signal-to-noise. If sufficiently improved, it may become sensitive enough to detect subtle blood-brain barrier leakage in a much shorter total scan time, lessening the burden on the patient and making it more suitable for use in the clinic. The choice of contrast agent also influences the sensitivity of the DCE-MRI experiment to subtle blood-brain barrier leakage. A smaller and more lipophilic molecule will more easily pass the blood-brain barrier, allowing for more contrast agent to extravasate causing larger signal differences between leaking and non-leaking tissue ${ }^{42}$. Recent research has also shown that gadolinium-based contrast agents may accumulate in the brain ${ }^{43}$. Although blood-brain barrier leakage of contrast agent occurs even in healthy subjects, actual long-term 
accumulation of the contrast agent was not anticipated in our work ${ }^{44}$. The effects of gadolinium accumulation from previous contrast agent injections should be considered in future studies using DCE-MRI, as it may affect the measurement. The analysis of the DCE-MRI data can also be significantly improved. In this thesis, the Patlak graphical approach was utilized as a pharmacokinetic model to distinguish between contrast agent in the blood and in the tissue ${ }^{45}$. However, the validity of this approach depends on the underlying assumptions on the behaviour of the contrast agent in vivo. For example, it assumes that once the contrast agent has left the blood pool, it does not "leak back" into the blood again (reflux), and is flow independent. If the leakage is indeed a passive process as is expected given the results in mouse models, this assumption may not be tenable ${ }^{46}$. However, due to the very low leakage rates, violation of this assumption probably has little influence on the outcome parameters of the model. Other, more advanced, pharmacokinetic models such as the two-compartment exchange model are available, which also take other physiological parameters into account, such as local perfusion, water (magnetization) exchange, and reflux. Although recent studies have shown that the Patlak graphical approach is suitable in low-leakage scenarios, a more advanced model may improve sensitivity and accuracy of the DCE-MRI measures, and provide more information on the underlying physiology differences caused by Alzheimer's disease ${ }^{8,47}$.

Several aspects of the ASL sequence can also be improved. First, it suffers from a low signal-to-noise, requiring many repeated measurements to accurately measure perfusion. Second, the resolution of the ASL sequence described in this thesis is also low at $3 \times 3 \times 7 \mathrm{~mm}^{3}$, and third, measuring the perfusion in the brain white matter is still challenging ${ }^{48}$. The recently published and widely supported whitepaper on the use of ASL will assist in standardizing protocols and the comparison of results between different studies $^{12}$. With sufficient improvements, ASL will provide a quick and accurate estimation of local perfusion, without the use of an endogenous contrast agent.

Diffusion MRI is widely used, but the diffusion sequence and the tractography methods could still be improved. A diffusion MRI sequence relies on a diffusion-weighted gradient along a certain axis to measure the diffusion of water in that direction. Measuring at different sensitization strengths and in more directions increases the amount of information available to reconstruct the distribution of the white matter axons. However, it is challenging to develop a sequence which gives enough information while still retaining a clinically feasible scan time. Improving the tractography procedures is 
also challenging. All tractography algorithms can accurately reconstruct the geometry of white matter fiber bundles if they are highly organized in one direction. However, more complex configurations such as crossing, bending, kissing, or fanning fibers still present a challenge and often lead to faulty reconstructions ${ }^{49}$. The constrained spherical deconvolution method does help in this regard, but other techniques may improve the tractography even further. In short, tractography can be very useful to investigate white matter connections, but it should be used with care, realizing that it is a model working under certain assumptions ${ }^{50}$. With further improved tractography methods, the reconstruction of the white matter pathways would likely be much more reliable and may assist in finding which pathways are most affected in Alzheimer's disease.

The MRI sequences used in this thesis have been able to detect group differences between patients with Alzheimer's disease and control subjects. If enough progress is made, it may also be possible in the future to develop sequences that can reliably differentiate between a healthy brain and a brain at risk for Alzheimer's disease, at a single subject level.

\section{Future directions}

Above all, future studies should validate the research presented in this thesis by repeating it in larger patient populations. There is also a need for further investigation of the blood-brain barrier leakage measures. First, to provide solid proof that DCE-MRI is able to provide a reliable and accurate measurement of very subtle leakage of the blood-brain barrier. This will be very difficult, as validation requires either a flow phantom that mimics the in vivo conditions, or a golden standard method that is proven to be able to accurately measure subtle blood-brain barrier leakage, both of which do not exist currently. Second, to discover what underlying process causes the leakage found with DCE-MRI.

To further investigate the role of the neurovascular unit in the pathophysiology of Alzheimer's disease, other pathways and elements of the neurovascular unit should be investigated. For example, cerebral water management is regulated by the blood-brain barrier, and may also be disturbed in Alzheimer's disease ${ }^{51}$. A possible way to measure water exchange is by using DCE-MRI51. Another possibility is to investigate local inflammation, which may be measured using $\mathrm{PET}^{52}$. However neuroinflammation is a complicated process and imaging of the different elements of the inflammation cascade is far from trivial ${ }^{53}$. Additionally, the blood-cerebrospinal fluid (CSF) barrier may also be 
of interest. The blood-CSF barrier mainly resides in the choroid plexus endothelial cells, and there are signs that it is also impaired in Alzheimer's disease ${ }^{54,55}$.

An important are of research on Alzheimer's disease aims to find the earliest marker to diagnose the disease. A reliable and early biomarker will not only make the patient aware of the future at an early stage, but it will also help to discover new treatments by enhancing our understanding of the underlying pathophysiology of Alzheimer's disease and to provide sensitive marker to test the efficacy of such treatments in clinical trials. The research discussed in this thesis introduces blood-brain barrier integrity as a possible future biomarker. Inserting the new information provided in this thesis into the hypothetical biomarker model of Jack et al. provides a single point (Figure 7.2) ${ }^{56}$. If longitudinal research can be performed on the blood-brain barrier integrity at different stages of Alzheimer's disease, this information may be used to extend this model. That should also provide information on the possible use of blood-brain barrier integrity as an early biomarker for Alzheimer's disease. Furthermore, if the blood-brain barrier integrity differs at different stages of the disease, this information may also be used to assess the effectiveness of new drugs. 


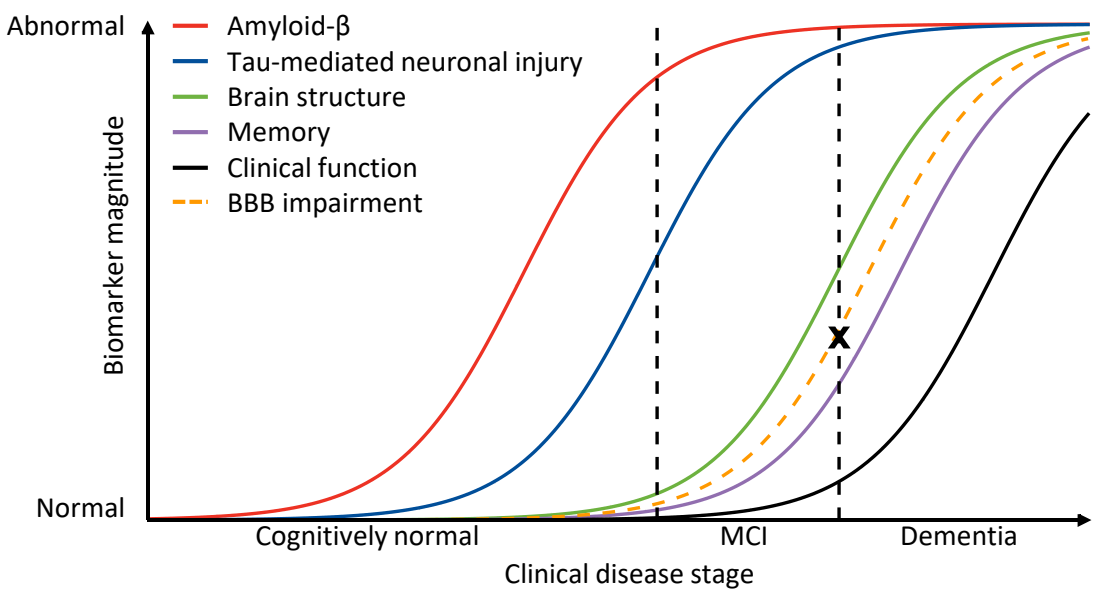

Figure 7.2: hypothetical biomarker model for the different stages of Alzheimer's disease ${ }^{56}$. The insights of this thesis regarding cerebrovascular pathology have been added to the graph in the form of a black " $X$ ". It is a single point due to the cross-sectional study design of the study described in this thesis. The patients either had a diagnosis of $\mathrm{MCl}$ or with early dementia due to Alzheimer's disease, which puts the data on the border between the two diagnoses. The biomarker magnitude of the point is defined by the symptoms of the patients and the relative difficulty with which the blood-brain barrier (BBB) impairment was detected. Clinical function was largely intact, and memory was compromised, but only slightly. The medial temporal lobe atrophy of the patients was significantly higher compared with the healthy controls, which would suggest the BBB impairment was more difficult to detect. The orange dashed line denotes a hypothetical curve of BBB impairment, based on the data presented in this thesis. However, it is very likely that the position of this line will vary from patient to patient, and BBB impairment may start at an earlier phase for some, and later for others. 


\section{Conclusion}

The main message of this thesis is that microvascular pathology plays a factor in Alzheimer's disease and that it can be measured using neuroimaging (MRI) methods. This message is not just meant for researchers, but also for healthcare professionals and the general public. The list of symptoms and signs which together define Alzheimer's disease has changed over the years. Note that the first patient diagnosed with Alzheimer's disease, Auguste $D$, also showed vascular pathology besides the plaques and tangles of insoluble proteins, but this aspect has received little attention in the last decades of research into Alzheimer's disease ${ }^{57}$. The most recent consensus on the diagnosis of Alzheimer's disease did not include a vascular component ${ }^{17}$. However, the cerebral microvascular pathology shown in this thesis and other studies, and the clear impact of vascular pathology on the prognosis of Alzheimer's disease, may indicate the need for an update of the definition of Alzheimer's disease. Perhaps it is time to recognize some cerebrovascular pathology, such as white matter lesions and microbleeds, as a normal part of Alzheimer's disease. The pathophysiology of Alzheimer's disease is likely a complicated cascade involving many elements which experience slow and progressive deterioration. Viewing the impact and deterioration of each element as unique features of the individual disease of a patient will help improve individual care and treatment. 


\section{References}

Lecrux C, Hamel E. The neurovascular unit in brain function and disease. Acta Physiol 2011; 203: 4759.

Abbott NJ, Patabendige AAK, Dolman DEM, Yusof SR, Begley DJ. Structure and function of the bloodbrain barrier. Neurobiol. Dis. 2010; 37: 13-25.

Ballabh P, Braun A, Nedergaard M. The blood-brain barrier: An overview: Structure, regulation, and clinical implications. Neurobiol Dis 2004; 16: 1-13.

ladecola C. The overlap between neurodegenerative and vascular factors in the pathogenesis of dementia. Acta Neuropathol 2010; 120: 287-296.

(1)

Broadwell RD, Sofroniew M V. Serum proteins bypass the blood-brain fluid barriers for extracellular entry to the central nervous system. Exp Neurol 1993; 120: 245-263.

Saunders NR, Liddelow SA, Dziegielewska KM. Barrier mechanisms in the developing brain. Front Pharmacol 2012; 3: 46.

Aerts HJWL, Jaspers K, Backes WH. The precision of pharmacokinetic parameters in dynamic contrast-enhanced magnetic resonance imaging: the effect of sampling frequency and duration. Phys Med Biol 2011; 56: 5665-5678.

Cramer SP, Larsson HBW. Accurate determination of blood-brain barrier permeability using dynamic contrast-enhanced T1-weighted MRI: a simulation and in vivo study on healthy subjects and multiple sclerosis patients. J Cereb Blood Flow Metab 2014; 34: 1655-65.

ladecola C. The Pathobiology of Vascular Dementia. Neuron 2013; 80: 844-866.

Zlokovic BV. Neurovascular mechanisms of Alzheimer's neurodegeneration. Trends Neurosci 2005; 28: 202-208.

Sagare AP, Bell RD, Zlokovic BV. Neurovascular dysfunction and faulty amyloid $\beta$-peptide clearance in Alzheimer disease. Cold Spring Harb Perspect Med 2012; 2. doi:10.1101/cshperspect.a011452.

Alsop DC, Detre J a., Golay X, Günther M, Hendrikse J, Hernandez-Garcia L et al. Recommended implementation of arterial spin-labeled perfusion MRI for clinical applications: A consensus of the ISMRM perfusion study group and the European consortium for ASL in dementia. Magn Reson Med 2015; 73: 102-116.

Prins ND, Scheltens P. White matter hyperintensities, cognitive impairment and dementia: an update. Nat Rev Neurol 2015; 11: 157-165.

Wardlaw JM, Valdés Hernández MC, Muñoz-Maniega S. What are white matter hyperintensities made of? Relevance to vascular cognitive impairment. J Am Heart Assoc 2015; 4: 001140.

Gouw AA, Seewann A, Vrenken H, Van Der Flier WM, Rozemuller JM, Barkhof F et al. Heterogeneity of white matter hyperintensities in Alzheimer's disease: Post-mortem quantitative MRI and neuropathology. Brain 2008; 131: 3286-3298.

Shulman RG, Rothman DL, Behar KL, Hyder F. Energetic basis of brain activity: Implications for neuroimaging. Trends Neurosci 2004; 27: 489-495.

McKhann GM, Knopman DS, Chertkow H, Hyman BT, Jack CR, Kawas $\mathrm{CH}$ et al. The diagnosis of dementia due to Alzheimer's disease: Recommendations from the National Institute on AgingAlzheimer's Association workgroups on diagnostic guidelines for Alzheimer's disease. Alzheimer's Dement 2011; 7: 263-269.

Hoffman JM, Welsh-Bohmer KA, Hanson M, Crain B, Hulette CM, Earl N et al. FDG PET imaging in patients with pathologically verified dementia. J Nucl Med 2000; 41: 1920-1928.

ladecola C. Neurovascular regulation in the normal brain and in Alzheimer's disease. Nat Rev 
Neurosci 2004; 5: 347-360.

Barker R, Ashby EL, Wellington D, Barrow VM, Palmer JC, Kehoe PG et al. Pathophysiology of white matter perfusion in Alzheimer's disease and vascular dementia. Brain 2014; 137: 1524-1532.

Hardy J. The amyloid hypothesis for Alzheimer's disease: A critical reappraisal. J Neurochem 2009; 110: 1129-1134.

Janota C, Lemere C a., Brito MA. Dissecting the Contribution of Vascular Alterations and Aging to Alzheimer's Disease. Mol Neurobiol 2015. doi:10.1007/s12035-015-9319-7.

De La Torre JC. Is Alzheimer's disease a neurodegenerative or a vascular disorder? Data, dogma, and dialectics. Lancet Neurol 2004; 3: 184-190.

Lyros E, Bakogiannis C, Liu Y, Fassbender K. Molecular Links Between Endothelial Dysfunction and Neurodegeneration in Alzheimer's Disease. Curr Alzheimer Res 2014; 11: 18-26.

Heppner FL, Ransohoff RM, Becher B. Immune attack: the role of inflammation in Alzheimer disease. Nat Rev Neurosci 2015; 16: 358-372.

Taheri S, Gasparovic C, Shah NJ, Rosenberg G a. Quantitative measurement of blood-brain barrier permeability in human using dynamic contrast-enhanced MRI with fast T1 mapping. Magn Reson Med 2011; 65: 1036-1042.

Taheri S, Gasparovic C, Huisa BN, Adair JC, Edmonds E, Prestopnik J et al. Blood-brain barrier permeability abnormalities in vascular cognitive impairment. Stroke 2011; 42: 2158-2163.

Scahill RI, Schott JM, Stevens JM, Rossor MN, Fox NC. Mapping the evolution of regional atrophy in Alzheimer's disease: unbiased analysis of fluid-registered serial MRI. Proc Natl Acad Sci U S A 2002; 99: 4703-7.

Braak H, Braak E. Staging of Alzheimer-related cortical destruction. Int Psychogeriatr 1997; 9 Suppl 1: 257-261

Yotter RA, Doshi J, Clark V, Sojkova J, Zhou Y, Wong DF et al. Memory decline shows stronger associations with estimated spatial patterns of amyloid deposition progression than total amyloid burden. Neurobiol Aging 2013; 34: 2835-2842.

Keaney J, Walsh DM, O'Malley T, Hudson N, Crosbie DE, Loftus T et al. Autoregulated paracellular clearance of amyloid- across the blood-brain barrier. Sci Adv 2015; 1: e1500472-e1500472.

Leinenga G, Götz J. Scanning ultrasound removes amyloid- $\beta$ and restores memory in an Alzheimer's disease mouse model. Sci Transl Med 2015; 7: 1-11.

Lorenz MW, Gonzalez M, Lienerth C, Loesel N, Thoelen N, Sitzer M. Influence of Temporal Insonation Window Quality on the Assessment of Cerebral Autoregulation with Transcranial Doppler Sonography. Ultrasound Med Biol 2007; 33: 1540-1545.

Stanimirovic DB, Friedman A. Pathophysiology of the neurovascular unit: disease cause or consequence? J Cereb Blood Flow Metab 2012; 32: 1207-1221.

Wardlaw JM, Smith C, Dichgans M. Mechanisms of sporadic cerebral small vessel disease: Insights from neuroimaging. Lancet Neurol 2013; 12: 483-497.

Lee C-W, Shih Y-H, Kuo Y-M. Cerebrovascular Pathology and Amyloid Plaque Formation in Alzheimer's Disease. Curr Alzheimer Res 2014; 11: 4-10.

Benarroch EE. Neurovascular unit dysfunction: A vascular component of Alzheimer disease? Neurology. 2008; 70: 1941.

Farrall AJ, Wardlaw JM. Blood-brain barrier: Ageing and microvascular disease - systematic review and meta-analysis. Neurobiol Aging 2009; 30: 337-352. 
a dementia risk score vs. the Framingham vascular risk scores. Neurology 2013; 80: 1300-1306.

40

41

Laske C, Stellos K. Vascular pathophysiology of Alzheimer's disease. Curr Alzheimer Res 2014; 11: 13.

Richard E, Schmand B, Eikelenboom P, Westendorp RG, Van Gool WA. The Alzheimer myth and biomarker research in dementia. J Alzheimers Dis 2012; 31 Suppl 3: S203-s209.

Seelig A, Gottschlich R, Devant RM. A method to determine the ability of drugs to diffuse through the blood-brain barrier. Proc Natl Acad Sci U S A 1994; 91: 68-72.

Kanda T, Fukusato T, Matsuda M, Toyoda K, Oba H, Kotoku J et al. Gadolinium-based Contrast Agent Accumulates in the Brain Even in Subjects without Severe Renal Dysfunction: Evaluation of Autopsy Brain Specimens with Inductively Coupled Plasma Mass Spectroscopy. Radiology 2015; 276: 228232.

Montagne A, Barnes SR, Sweeney MD, Halliday MR, Sagare AP, Zhao Z et al. Blood-Brain Barrier Breakdown in the Aging Human Hippocampus. Neuron 2015; 85: 296-302.

Patlak CS, Blasberg RG, Fenstermacher JD. Graphical evaluation of blood-to-brain transfer constants from multiple-time uptake data. J Cereb Blood Flow Metab 1983; 3: 1-7.

Nitta T, Hata M, Gotoh S, Seo Y, Sasaki H, Hashimoto N et al. Size-selective loosening of the bloodbrain barrier in claudin-5-deficient mice. J Cell Biol 2003; 161: 653-660.

Barnes SR, Ng TSC, Montagne A, Law M, Zlokovic BV., Jacobs RE. Optimal acquisition and modeling parameters for accurate assessment of low $\mathrm{K}$ trans blood-brain barrier permeability using dynamic contrast-enhanced MRI. Magn Reson Med 2016; 75: 1967-1977.

Van Osch MJP, Teeuwisse WM, Van Walderveen M a a, Hendrikse J, Kies D a., Van Buchem M a. Can arterial spin labeling detect white matter perfusion signal? Magn Reson Med 2009; 62: 165-173.

Jbabdi S, Johansen-Berg H. Tractography: Where Do We Go from Here? Brain Connect 2011; 1: 169183.

Jones DK, Knösche TR, Turner R. White matter integrity, fiber count, and other fallacies: The do's and don'ts of diffusion MRI. Neuroimage 2013; 73: 239-254.

Anderson VC, Lenar DP, Quinn JF, Rooney WD. The Blood-Brain Barrier and Microvascular Water Exchange in Alzheimer's Disease. Cardiovasc Psychiatry Neurol 2011; 2011: 1-9.

Wu C, Li F, Niu G, Chen X. PET imaging of inflammation biomarkers. Theranostics 2013; 3: 448-466.

Jacobs AH, Tavitian B. Noninvasive molecular imaging of neuroinflammation. J Cereb Blood Flow Metab 2012; 32: 1393-415.

Engelhardt B, Sorokin L. The blood-brain and the blood-cerebrospinal fluid barriers: Function and dysfunction. Semin Immunopathol 2009; 31: 497-511.

Starr JM, Farrall AJ, Armitage P, McGurn B, Wardlaw JM. Blood-brain barrier permeability in Alzheimer's disease: a case-control MRI study. Psychiatry Res - Neuroimaging 2009; 171: 232-241.

Jack CR, Knopman DS, Jagust WJ, Shaw LM, Aisen PS, Weiner MW et al. Hypothetical model of dynamic biomarkers of the Alzheimer's pathological cascade. Lancet Neurol 2010; 9: 119-128.

De La Torre JC. Is Alzheimer's disease preceded by neurodegeneration or cerebral hypoperfusion? Ann Neurol 2005; 57: 783-784. 


\section{Summary}

Alzheimer's disease $(A D)$ is the most common cause of cognitive decline and dementia in the elderly. Increasing evidence supports that vascular pathology may play an important role in the pathophysiology of AD. However, the pathways through which vascular pathology would cause neurodegeneration in $A D$ are unclear. Neuroimaging, in the form of advanced Magnetic Resonance Imaging (MRI) methods, may assist in revealing the role of vascular pathology, although the effects are hypothesized to represent small changes which may be hard to detect. MRI sequences generally used in $A D$ are mainly aimed at detecting (macro)structural brain abnormalities such as atrophy and excluding other conditions that may cause the symptoms. However, these sequences cannot be used to detect microvascular abnormalities. Hardware and analysis methods for MRI sequences have improved to a point that these small changes may become detectable by using advanced techniques. The main aim of this thesis was to apply several of these techniques in patients with an early stage of $A D$ and control subjects to study cerebrovascular pathology in early AD. Specifically, Dynamic Contrast-Enhanced (DCE-)MRI was used to assess the blood-brain barrier (BBB), Arterial Spin Labeling (ASL) was used to asses local perfusion, and diffusion MRI was used to assess hippocampal structural connectivity of the white matter and its relation to white matter hyperintensities (WMHs).

In Chapter 1, a general overview on AD, its known link with vascular pathology, and the neuroimaging techniques applied in this thesis are given. Furthermore, a short description of the aim, research questions and contents of the chapters of this thesis are provided.

In Chapter 2, a literature review is presented to investigate different ways through which the BBB can be investigated non-invasively and in vivo. The results showed that multiple techniques can be used, but that the most common technique, the measurement of the concentration of albumin protein in the cerebrospinal fluid relative to that in the blood, does not provide information on the spatial extent or location of the BBB impairment. Neuroimaging techniques were also applied in $A D$, but it was found that most studies focused on patients who already exhibited cerebrovascular pathology, and results of $B B B$ impairment in pure $A D$ without any vascular pathology were scarce. It was also found that the most promising techniques to measure BBB impairment in $A D$ in a spatially resolved way are to apply neuroimaging techniques with a contrast agent. 
In Chapter 3, a pilot study was conducted, in which a novel dual-time resolution DCEMRI technique was applied in 16 patients with early $A D$ and 17 healthy controls. The dual-time resolution protocol allowed for a proper separation of signal arising from contrast agent in the blood and the contrast agent which has leaked through the BBB into the brain parenchyma, although a total scan time of approximately 25 minutes was used. Using a pharmacokinetic model, the BBB leakage rate $K_{i}$ was calculated. It was shown that the leakage rate was very low (order of $10^{-4} \mathrm{~min}^{-1}$ ) and difficult to measure. The patients showed a significantly higher $\mathrm{K}_{\mathrm{i}}$ in the cortex, but not in other brain regions. However, a histogram analysis allowed for the calculation of the total (spatial) leakage extent, defined as the fraction of detectably leaking voxels VL. The VL proved more sensitive to detect group differences, and the patients showed a greater extent of BBB leakage compared with the controls both in the gray matter and white matter. The leakage extent was also found to be associated with a score for global cognition, indicating a progressive process of $B B B$ leakage in $A D$.

In Chapter 4, several methods to increase the sensitivity of a DCE-MRI experiment to subtle BBB leakage were explored. This was necessary to find ways to decrease the burden of the scan on the patient while retaining the possibility of measuring very subtle BBB leakage. The investigated methods included varying the total scan time and the application of a noise suppression method in combination with a measure for leakage extent. Computer simulations were combined with data from in vivo experiments to assess the effects of changing the scan time and applying the noise reduction method. By using a distribution of leakage rate values rather than single leakage rates in the computer simulations, realistic effects of noise and in vivo variations of the leakage could be investigated. It was found that the measured leakage rate differed from the true leakage rate, and that it depended on the total scan time. However, it was also found that measuring the differences between a group of patients exhibiting subtle BBB leakage and healthy controls was feasible at shorter scan times with proper statistical power, given a large enough group and effect size. For example, the statistical power calculations showed that with group sizes of 15 , a scan time of 15 minutes is sufficient to detect differences in $\mathrm{V}_{\mathrm{L}}$ in the cortex.

In Chapter 5, the results of the DCE-MRI experiment were combined with the ASL results. Both local perfusion and the permeability of the microvascular wall to contrast agent is tightly regularized by the neurovascular unit, a collection of cells and subcellular structures surrounding the brain capillaries which support and protect the neurons. 
Therefore, differences in local perfusion and BBB permeability between healthy controls and patients with early $A D$ point towards dysfunction of the neurovascular unit. Therefore, combining the DCE-MRI results with ASL allowed for the simultaneous investigation of different aspects of the cerebral microvessels. The results showed BBB leakage and global hypoperfusion. Furthermore, it was found that the hypoperfusion and increased BBB leakage were correlated. This suggests that the AD pathology likely interacts with multiple elements of the neurovascular unit.

In Chapter 6, microvascular pathology in $A D$ was investigated by using diffusion MRI to study the impact of WMHS on the white matter connections. WMHs are hyperintense regions on Fluid-Attenuated Inversion Recovery (FLAIR) MRI scans of the brain, and are frequently found in patients with $A D$. They are considered pathological manifestations of vascular origin, and are associated with cognitive decline in AD. Demyelination is a common histological finding in WMHs, and it is likely that WMHs affect the white matter connections between different cortical regions. Using diffusion MRI in combination with tractography, the structural white matter connections were reconstructed and hippocampal connections were investigated in patients with subjective memory complaints, Mild Cognitive Impairment $(\mathrm{MCl}$, a prodromal form of $\mathrm{AD}$ ) and $\mathrm{AD}$. It was found that white matter tracts were relatively larger when they incorporated more WMHs. This increase in volume might indicate either a pathological process through swelling of the white matter, or a compensatory process through a rerouting of the white matter tracts to avoid the WMHs.

In Chapter 7, the results of the research of this thesis are discussed in a broader perspective, and the research questions of the thesis, which were introduced in Chapter 1, are answered. Furthermore, the conceptual background, clinical implications, strengths and limitations, possible future directions, and a general conclusion are presented.

Overall, the advanced MRI methods described in this thesis were used to find differences between patients with $A D$ and controls on a group level, to gain further insight into the pathophysiology of $A D$. Further improvement of these techniques will allow for the detection of even more subtle pathology. Primarily the DCE-MRI sequence may be improved to shorten the scan time, decrease sensitivity to noise and imaging artefacts, and acquire whole-brain images to make it clinically feasible. With sufficient improvements it may ultimately become possible to apply these techniques to diagnose $A D$ on an individual level at an early stage. 


\section{Samenvatting}

De ziekte van Alzheimer is de meest voorkomende oorzaak van cognitieve achteruitgang en dementie bij ouderen. $\mathrm{Er}$ is steeds meer bewijs dat aantoont dat vasculaire pathologie, naast neurodegeneratie, een belangrijke rol speelt in de pathofysiologie van de ziekte van Alzheimer. De relatie tussen vasculaire pathologie en neurodegeneratie in de ziekte van Alzheimer is nog steeds onduidelijk. Medische beeldvorming, en dan met name het gebruik van geavanceerde Magnetic Resonance Imaging (MRI), kan helpen bij het onderzoek naar de rol van vasculaire pathologie, alhoewel de veronderstelde effecten waarschijnlijk klein en moeilijk te detecteren zijn. Algemeen toegepaste MRI sequenties bij de ziekte van Alzheimer zijn vooral gericht op het opsporen van (macro) structurele afwijkingen in de hersenen, zoals atrofie, en worden daarnaast ook gebruikt om andere aandoeningen uit te sluiten die de symptomen kunnen veroorzaken. Deze sequenties kunnen echter niet worden gebruikt om microvasculaire afwijkingen te detecteren. In de loop der tijd zijn MRI hardware en analysemethoden sterk verbeterd, waardoor deze kleine veranderingen gedetecteerd kunnen worden met behulp van geavanceerde technieken. Het belangrijkste doel van dit proefschrift was om een aantal van deze technieken toe te passen bij patiënten met een vroeg stadium van de ziekte van Alzheimer en controle proefpersonen, om zo cerebrovasculaire pathologie te bestuderen in het beginstadium van de ziekte. Dynamic Contrast-Enhanced (DCE) MRI werd gebruikt om de integriteit van de bloed-hersenbarrière (BBB) te bepalen, Arterial Spin Labeling (ASL) werd toegepast om lokale perfusie te meten, en diffusie MRI werd ingezet om de hippocampale structurele connectiviteit van de witte stof te beoordelen, vooral in relatie tot witte stof afwijkingen.

Hoofdstuk 1 geeft een algemene inleiding over de ziekte van Alzheimer en het verband met vasculaire pathologie, en een overzicht van de MRI technieken die worden toegepast in dit proefschrift. Daarna volgt een korte beschrijving van het doel, de onderzoeksvragen, en de inhoud van de hoofdstukken.

Hoofdstuk 2 bevat een literatuuroverzicht van de verschillende technieken waarmee de integriteit van de BBB kan worden gemeten op een non-invasieve manier en in vivo. De resultaten van het literatuuronderzoek gaven aan dat er verschillende technieken kunnen worden gebruikt. De meest gebruikte techniek is het meten van de albumine ratio. Bij deze techniek wordt de concentratie van het eiwit albumine in de cerebrospinale vloeistof vergeleken met die in het bloed. Helaas geeft de albumine ratio 
geen informatie over de omvang of locatie van de BBB schade. Om BBB schade te meten in de ziekte van Alzheimer zijn ook beeldvormingstechnieken toegepast, die uitwezen dat er waarschijnlijk BBB lekkage aanwezig is bij mensen met de ziekte. Het bleek ook dat de meeste studies gericht waren op patiënten die al op een andere wijze cerebrovasculaire pathologie vertoonden, en resultaten van BBB schade in de mensen met de ziekte van Alzheimer zonder vasculaire pathologie waren schaars. Daarnaast bleek dat het mogelijk is om BBB schade te meten op een manier die het mogelijk maakt om de locatie in het brein en de distributie van de schade vast te stellen. De meest veelbelovende technieken hiervoor zijn beeldvormingstechnieken waarbij een contrastmiddel wordt gebruikt.

Hoofdstuk 3 bevat de resultaten van een studie waarin een DCE-MRI techniek met dubbele tijdsresolutie werd toegepast in 16 patiënten met vroege ziekte van Alzheimer en 17 gezonde ouderen. De dubbele tijdsresolutie maakte het mogelijk om het signaal van het contrastmiddel in het bloed te scheiden van het signaal van het contrastmiddel dat door de BBB heen is gelekt naar het hersenweefsel. Daarvoor was wel een totale scantijd van ongeveer 25 minuten nodig. Met behulp van een farmacokinetisch model werd de BBB lekkagemaat $K_{i}$ berekend. Deze lekkage bleek erg laag (ongeveer $10^{-4} \mathrm{~min}^{-1}$ ) en moeilijk te meten. De patiënten hadden een significant hogere $K_{i}$ in de cortex, maar er waren geen significante verschillen in andere hersengebieden zoals de diepe grijze stof of witte stof. Het toepassen van een histogram analysemethode maakte het mogelijk om een nieuwe lekkagemaat te ontwikkelen. Deze lekkagemaat is gedefinieerd als de fractie van het aantal detecteerbaar lekkende voxels $\mathrm{VL}$, uitgedrukt als een relatief volume ten opzichte van het totale hersenweefselvolume. De $v_{\llcorner}$bleek gevoeliger de gemiddelde lekkage $K_{i}$ voor verschillen tussen de groepen, en de patiënten vertoonden hiermee een sterkere mate van BBB lekkage dan de controles, zowel in de grijze als witte stof. De lekkagemaat vL bleek ook toe te nemen naarmate de globale cognitie (gemeten met een algemeen neuropsychologisch onderzoek) slechter werd. Dit suggereert dat BBB lekkage in de ziekte van Alzheimer een progressief proces is, omdat de BBB lekkage erger wordt naarmate de ziekte vordert.

Hoofdstuk 4 beschrijft een onderzoek naar verschillende manieren om de gevoeligheid van een DCE-MRI experiment voor subtiele BBB lekkage te verhogen. Het DCE-MRI protocol duurt lang, ongeveer 25 minuten, wat erg belastend kan zijn voor een patiënt. Als de gevoeligheid van het protocol vergroot kan worden, kan de scan makkelijker op grotere schaal worden toegepast. Om de gevoeligheid te vergroten zijn verschillende 
manieren onderzocht, namelijk het variëren van de totale scantijd, en de toepassing van een methode om ruis te onderdrukken in combinatie met de $\mathrm{V}_{\mathrm{L}}$ maat voor de fractie van lekkende voxels. Computersimulaties zijn gecombineerd met de data van in vivo experimenten om de effecten van het veranderen van de scantijd en het toepassen van de ruisonderdrukking beoordelen. Door het gebruik van een verdeling van lekkagewaarden in de computersimulaties, in tegenstelling tot het gebruik van een enkele lekkagewaarde, konden realistische effecten van ruis en in vivo variaties van de lekkage worden onderzocht. Het bleek dat de gemeten lekkage verschilde van de lekkage die als input was gebruikt, en dat de grootte van het verschil afhing van de totale scantijd. Er werd ook vastgesteld dat er genoeg onderscheidend vermogen was om verschillen te kunnen meten tussen een groep patiënten met subtiele BBB lekkage en gezonde controles bij kortere scantijden, onder voorwaarde dat de groepsgrootte en de grootte van het effect voldoende waren. Zo wezen statistische berekeningen bijvoorbeeld uit dat bij een groepsgrootte van 15, een scantijd van 15 minuten voldoende was om verschillen in $V_{L}$ in de cortex te kunnen detecteren, mits die verschillen ongeveer even groot waren als de verschillen die in de proefpersonen waren gevonden.

Hoofdstuk 5 beschrijft de resultaten van een onderzoek naar de integriteit van de neurovasculaire unit in de ziekte van Alzheimer. De neurovasculaire unit is een verzameling van cellen en subcellulaire structuren in en rondom de vaatwand van de capillairen in de hersenen. Deze structuren bieden ondersteuning aan de neuronen en beschermen ze tegen neurotoxische stoffen die zich in de bloedbaan bevinden. Zowel de bloedperfusie als de permeabiliteit van de BBB worden streng gereguleerd door de neurovasculaire unit, en kunnen worden gemeten met behulp van ASL en DCE-MRI. Daarom zijn deze technieken gezamenlijk toegepast in patiënten met een vroege vorm van de ziekte van Alzheimer en gezonde controles. Uit de resultaten van dit onderzoek bleek dat de patiënten globale hypoperfusie en BBB lekkage vertoonden vergeleken met de gezonde controles. Daarnaast werd een correlatie tussen de hypoperfusie en de verhoogde BBB lekkage gevonden. Dit suggereert dat de achterliggende pathofysiologie van de ziekte van Alzheimer invloed heeft op de neurovasculaire unit als geheel, in plaats van op losse functionele onderdelen zoals de BBB.

In hoofdstuk 6 worden de resultaten besproken van het toepassen van diffusie MRI om de impact van witte stof afwijkingen (Witte Matter Hyperintensities, WMHs) op de witte stof-verbindingen in de ziekte van Alzheimer te bestuderen. De WMHs zijn hyperintense 
gebieden op Fluid-Attenuated Inversion Recovery (FLAIR) MRI-scans van de hersenen, en zijn een veelvoorkomende bevinding bij patiënten met de ziekte van Alzheimer. De etiologie van WMHs is niet geheel duidelijk, maar het wordt vaak aangenomen dat ze worden veroorzaakt door vasculaire pathologie. Daarnaast worden ze geassocieerd met cognitieve achteruitgang in ziekte van Alzheimer. Door middel van histologie is demyelinisatie gevonden in WMHs, en het is waarschijnlijk dat ze de witte stof banen tussen verschillende corticale gebieden beïnvloeden. Door het toepassen van diffusie MRI in combinatie met tractografie zijn de witte stof banen gereconstrueerd en zijn de hippocampale verbindingen van patiënten met subjectieve geheugenklachten, Mild Cognitive Impairment ( $\mathrm{MCl}$, een prodromale vorm van ziekte van Alzheimer) en de ziekte van Alzheimer onderzocht. Het bleek dat de witte stof banen relatief groter waren als er veel WMHs in voor kwamen. Dit was een verassend resultaat aangezien atrofie een van de kenmerkende verschijnselen is in de ziekte van Alzheimer. Deze toename in volume duidt op verminderde atrofiëring van het weefsel, en kan ofwel door zwelling komen als gevolg van een pathologisch proces, of een compensatieproces zijn dat aangeeft dat de witte stof banen veranderen om de WMHs te omzeilen.

In hoofdstuk 7 worden de resultaten van het onderzoek van dit proefschrift besproken in een breder kader, en worden de onderzoeksvragen uit hoofdstuk 1 beantwoord. Daarnaast worden de klinische implicaties, sterke punten en beperkingen van het onderzoek, mogelijke toekomstige onderzoeksrichtingen, en een algemene conclusie beschreven.

De geavanceerde MRI methoden beschreven in dit proefschrift zijn gebruikt om verschillen te zoeken tussen patiënten met de ziekte van Alzheimer en controles op groepsniveau. Het doel hiervan was om meer inzicht te krijgen in de pathofysiologie van de ziekte van Alzheimer. De verschillende MRI technieken kunnen nog verbeterd worden in de toekomst. Mogelijke verbeteringen van de DCE-MRI sequentie kunnen bijvoorbeeld de scanduur verkorten, de gevoeligheid voor ruis en beeldvormingsartefacten verminderen, en het bereik van de sequentie vergroten zodat BBB lekkage over de gehele hersenen kan worden gemeten. Pas als deze technieken verder worden verbeterd, zal het detecteren van de subtiele pathologie mogelijk worden in individuele patiënten, en kunnen de technieken in een klinische setting gebruikt worden. 


\section{Samenvatting voor niet-deskundigen}

Centraal in dit proefschrift staan de kleine bloedvaten in de hersenen van mensen met de ziekte van Alzheimer. De ziekte van Alzheimer is de meest voorkomende oorzaak van achteruitgang van het verstandelijk vermogen en dementie onder ouderen in Nederland. Ondanks vele tientallen jaren onderzoek is het nog steeds niet bekend welke onderliggende ziekteprocessen de ziekte van Alzheimer veroorzaken. Tot nu toe werden ophopingen van eiwitten in de hersenen, de zogenaamde plaques, als hoofdoorzaak gezien, en het onderzoek naar de ziekte van Alzheimer is daar dan ook vooral op toegespitst. Helaas heeft dat nog niet tot een duidelijke oorzaak van het ziektebeeld geleid, en het onderzoek heeft ook nog geen medicijn tegen de ziekte opgeleverd. Er zijn steeds meer aanwijzingen dat hart- en vaatziekten de kleine bloedvaten in de hersenen aantasten. Om die reden, was het doel van het onderzoek in dit proefschrift om de kleine bloedvaten in de hersenen van mensen met de ziekte van Alzheimer te onderzoeken.

De bloedvaten in de hersenen zijn anders dan elders in het lichaam. De kleinste haarvaatjes die het hersenweefsel doorkruisen zijn namelijk gespecialiseerd om de hersencellen optimaal te kunnen ondersteunen en beschermen. Daarom hebben deze bloedvaatjes, in tegenstelling tot die in de rest van het lichaam, een bijzondere vaatwand die alle transport van stoffen van en naar het omliggende weefsel streng reguleert. Deze vaatwand bestaat uit meerdere soorten cellen en structuren, die samen de zogenaamde bloed-hersenbarrière vormen. De bloed-hersenbarrière zorgt ervoor dat de hersencellen voldoende voedingsstoffen krijgen om te kunnen blijven werken. Het zorgt er ook voor dat andere ongewenste stoffen die schadelijk kunnen zijn voor de hersencellen strikt in de bloedbaan worden gehouden, en niet de hersencellen kunnen bereiken.

Om de haarvaten in de hersenen te kunnen onderzoeken is voor het onderzoek in dit proefschrift gebruik gemaakt van Magnetic Resonance Imaging (MRI). Met een MRI scanner kunnen afbeeldingen gemaakt worden van het lichaam, waaronder de hersenen. De MRI scanner is een veelzijdig apparaat dat met verschillende technieken een breed scala aan hersenfoto's kan genereren, die gebruikt kunnen worden om verschillende soorten afwijkingen detecteren. Zo zijn er bijvoorbeeld scans die de lokale doorbloeding kunnen meten, en scans die hersenactiviteit kunnen meten. 
In Hoofdstuk 1 is een korte introductie over de ziekte van Alzheimer en de rol van vaatziekten daarin gegeven. Het blijkt dat de aanwezigheid van hart- en vaatziekten ook de kans op de ziekte van Alzheimer vergroten, en ook de achteruitgang van de patiënt kunnen verergeren. Daarnaast is het zo dat bij mensen met de ziekte van Alzheimer bijna altijd ook een hart- of vaatziekte blijkt te zijn, de hersendoorbloeding verlaagd is, en er afwijkingen zijn in de kleine bloedvaten in de hersenen. Die afwijkingen bestaan vooral uit kleine ontstekingen rond de bloedvaten en verstoord transport van stoffen van en naar het breinweefsel, wat kan wijzen op problemen met de bloed-hersenbarrière.

Door middel van het bestuderen van de huidige wetenschappelijke literatuur is eerst vastgesteld of al bekend is of er mogelijk schade is aan de bloed-hersenbarrière in mensen met de ziekte van Alzheimer. De uitkomsten van dit literatuuronderzoek zijn besproken in Hoofdstuk 2. Uit de literatuur bleek dat de bloed-hersenbarrière waarschijnlijk beschadigd is in de ziekte van Alzheimer. Het bewijs hiervoor kwam hoofdzakelijk van studies waarbij de concentratie van het eiwit albumine in het bloed en in het hersenvocht is gemeten. Als er teveel albumine in het hersenvocht zit vergeleken met de hoeveelheid albumine in het bloed, dan is de kans groot dat het albumine door de bloed-hersenbarrière is gelekt. Helaas kan deze methode alleen gebruikt worden om te meten óf het lekt, maar kan het niet gebruikt worden om te achterhalen waar de schade zich bevindt. Er zijn technieken waarbij het meten en bepalen van de locatie van de schade wel mogelijk is. Deze technieken maken meestal gebruik van een methode om het brein in beeld te brengen in combinatie met een contrastmiddel. De mate waarin het contrastmiddel dan de bloedbaan verlaat en in het weefsel komt is dan een maat voor bloed-hersenbarrière lekkage. Het bleek ook dat deze methodes zelden waren toegepast bij mensen met de ziekte van Alzheimer.

Omdat er meer informatie nodig was over de bloed-hersenbarrière in de ziekte van Alzheimer, is er een onderzoek opgestart waarbij één van de meest veelbelovende technieken werd toegepast bij mensen met een vroeg stadium van deze ziekte. De opzet en resultaten van dit onderzoek zijn besproken in Hoofdstuk 3. De techniek die is gebruikt heet contrast-versterkte MRI. Tijdens het contrast-versterkte MRI onderzoek wordt een contrastmiddel ingespoten in de bloedbaan. Door continu MRI-beelden te blijven maken van het brein kan de aankomst en verspreiding van het contrastmiddel in beeld gebracht worden als een soort film. Aangezien het contrastmiddel niet door de bloed-hersenbarrière zou mogen gaan en dus in de bloedbaan zou moeten blijven, is de 
aanwezigheid van contrastmiddel in het breinweefsel een teken dat de bloedhersenbarrière lek is. Met behulp van contrast-versterkte MRI is aangetoond dat de bloed-hersenbarrière bij mensen met een vroeg stadium van de ziekte van Alzheimer meer contrastmiddel doorlaat dan bij gezonde ouderen. Daarnaast bleek de mate van lekkage proportioneel aan de achteruitgang van het verstandelijk vermogen, wat aangeeft dat de lekkage erger wordt naarmate het ziekteproces vordert.

De gemeten bloed-hersenbarrière lekkage was zeer subtiel, en een speciaal daarvoor aangepaste vorm van contrast-versterkte MRI was noodzakelijk om het toch te kunnen detecteren. In Hoofdstuk 4 zijn verschillende mogelijke aanpassingen aan het contrastversterkte MRI onderzoek besproken, die het onderzoek gevoeliger kunnen maken voor deze zeer subtiele lekkage. Voorbeelden van zulke aanpassingen zijn het toepassen van een methode om de ruis op de MRI beelden te schatten en te verwijderen, en de effecten van het langer meten van de verspreiding van het contrastmiddel. Uit dit onderzoek bleek dat de gemeten lekkage sterk wordt beïnvloed door ruis op de beelden. Dat is ook de reden dat onderscheid maken tussen het brein van een patiënt met de ziekte van Alzheimer en een gezond persoon nog niet mogelijk is. Daarnaast is onderzocht wat de kans is dat verschillen in bloed-hersenbarrière lekkage tussen een groep patiënten met de ziekte van Alzheimer en een controlegroep door toeval komen. Het bleek dat deze kans klein genoeg is om te kunnen stellen dat er daadwerkelijk verschillen zijn tussen de groepen, alhoewel deze kans wel afhangt van de grootte van de groepen en het verschil in lekkage.

Op de wanden van de kleine bloedvaatjes in de hersenen zitten spiercellen, die de lokale bloedtoevoer reguleren. Deze spiercellen, samen met de bloed-hersenbarrière en andere ondersteunende cellen, vormen de neurovasculaire unit. De neurovasculaire unit is een naam voor de intensieve samenwerking van de zojuist genoemde cellen, en heeft als doel te zorgen dat de neuronen in de hersenen alles krijgen om optimaal kunnen functioneren. De neurovasculaire unit regelt de toevoer en afvoer van bloed, energie, en essentiële stoffen. Met behulp van de MRI techniek Arteriële Spin Labeling (ASL), kan de lokale bloedtoevoer in het brein gemeten worden zonder dat er contrastmiddel ingespoten hoeft te worden. In Hoofdstuk 5 zijn de ASL en contrast-versterkte MRI technieken gecombineerd, om zo te kunnen bepalen of de werking van verschillende elementen van de neurovasculaire unit in mensen met de ziekte van Alzheimer even goed is als bij gezonde controles. Uit de resultaten bleek dat mensen met de ziekte van Alzheimer een verminderde doorbloeding van de hersenen hebben, en dat de mate van 
verminderde doorbloeding proportioneel is aan de lekkage van de bloed-hersenbarrière. Dat wijst erop dat in de ziekte van Alzheimer de gehele neurovasculaire unit is aangetast, en niet alleen de werking van losse onderdelen.

Op MRI hersenscans van mensen met de ziekte van Alzheimer zijn vaak abnormale witte vlekken te zien. Deze vlekken bevinden zich in de witte stof van het brein, en worden daarom ook wel witte stof hyperintensiteiten genoemd. Naast de witte stof bevat het brein ook grijze stof, ook wel bekend als de hersenschors. Alhoewel er nog niet heel veel bekend is over deze witte stof hyperintensiteiten, is wel vastgesteld dat ze een uiting zijn van schade aan de witte stof, en dat deze schade waarschijnlijk is veroorzaakt door een langdurig bloedtoevoerprobleem. Daarom is het aannemelijk dat witte stof hyperintensiteiten een symptoom zijn van vaatschade in de hersenen. De witte stof van de hersenen bestaat uit zenuwcellen die verschillende delen van de hersenschors met elkaar verbinden, ofwel de bekabeling van het brein. De hersenschors is onder andere verantwoordelijk voor het denk- en rekenwerk, en de witte stof zorgt ervoor dat de verschillende stukken hersenschors goed kunnen samenwerken. Met behulp van diffusiegewogen MRI kunnen de witte stofbanen in beeld worden gebracht. In Hoofdstuk 6 is diffusie-gewogen MRI toegepast in mensen met verschillende maten van geheugenklachten, waaronder ook mensen met de ziekte van Alzheimer. De witte stofbanen van en naar de hippocampus, het gedeelte van de hersenschors dat verantwoordelijk is voor geheugen, zijn gereconstrueerd, en vervolgens is bekeken wanneer deze banen door witte stof hyperintensiteiten gingen. Het bleek dat als bij mensen de banen door meer witte stof hyperintensiteiten gingen, ze ook relatief groter werden. Een mogelijke verklaring is dat de witte stof hyperintensiteiten schade vertegenwoordigen die zwelling van de witte stofbanen veroorzaakt. Een andere mogelijke verklaring is dat de witte stofbanen de witte stof hyperintensiteiten gaan omzeilen, om zo de schade in de witte stof hyperintensiteiten te vermijden. Verder onderzoek zal moeten uitwijzen wat de verklaring voor deze bevinding is.

In Hoofdstuk 7 worden conclusies getrokken uit de resultaten van het proefschrift. Ook worden de resultaten vanuit verschillende perspectieven besproken, en worden aanbevelingen gedaan voor toekomstig onderzoek. 


\section{Valorization Addendum}

\section{Healthcare problem}

Alzheimer's Disease (AD) is the most common cause of cognitive decline and dementia in the elderly in the developed world. It is characterized by an insidious onset and slow deterioration of cognitive function, progressing until death. The impact of $A D$ on daily life is enormous, both for the patient and loved ones. Increasing standards of living and healthcare also increases the prevalence of $A D$ in developing countries as people live longer, and it is estimated that in 2040 roughly 90 million people worldwide will suffer from $A D$, making it a global healthcare problem.

Despite the fact that AD has been identified and investigated since 1906, research efforts still have not been able to find the cause of $A D$, nor is a cure currently available. The main focus of $A D$ research has been the accumulations of insoluble proteins amyloid $B$ causing plaques, and $\tau$ causing tangles in the brains of patients with AD. However, treatments aimed at preventing or removing the plaques and tangles have so far not yielded a proper treatment for the symptoms of AD. Furthermore, biomarkers which aim for early diagnosis of $A D$, have so far shown mixed results, and sensitively and reliably diagnosing $A D$ at an early (presymptomatic) state is not possible. This has prompted some researchers to search for alternate explanations that might cause AD. One of the most promising candidates is vascular pathology. It has been shown that patients exhibiting pure $A D$ without vascular pathology are rare, and many risk factors have a vascular component or basis. A prime suspect for vascular pathology in the brain is the neurovascular unit, a structure located in the microvascular wall and unique to the brain, which is specialized to support and protect neuronal function.

\section{Main findings}

The main results of this thesis is that different elements of the neurovascular unit are damaged in AD. The blood-brain barrier is an important part of the neurovascular unit which strictly regulates transport between the blood and brain tissue. Using Dynamic Contrast-Enhanced (DCE) MRI, the blood-brain barrier was found to be impaired in AD, allowing small contrast agent molecules to leak out of the blood space. Although the consequences of the blood-brain barrier impairment are currently unclear, the uncontrolled passage of substances into the vulnerable brain parenchyma is very likely to be harmful. The research in this thesis also aims to assist future research, as possible 
improvements to the DCE-MRI sequence and analysis have been investigated, making it more sensitive to the subtle blood-brain barrier leakage found in AD. Combining the DCEMRI technique with Arterial Spin Labeling (ASL) MRI not only showed that patients with an early stage of $A D$ exhibit cerebral hypoperfusion, but that the hypoperfusion is also linked to the blood-brain barrier leakage severity. Given that local perfusion is also regulated by the neurovascular unit, this shows that $A D$ affects multiple elements of the neurovascular unit, and that the neurovascular unit impairment is progressive.

Another aspect of cerebrovascular pathology are White Matter Hyperintensities (WMHs), which are a common finding on brain MRI images of patients with AD. WMHs are considered to represent vascular pathology, and often exhibit local pathological effects such as demyelination. Using diffusion MRI, the research in this thesis has shown that the WMHs exhibit a direct effect on the hippocampal white matter bundles connecting the hippocampi with different gray matter regions. Specifically, it was found that WMHs cause an enlargement of the white matter tracts going through them. This indicates either a pathological swelling of the white matter tracts, or a compensation mechanism where white matter fibers are rerouted to avoid WMHs. Both hypothesized mechanisms could have a detrimental effect on information transfer speed, and consequently cognitive function.

Combined, the research in this thesis provides additional evidence and possible pathways for cerebrovascular pathology in AD. Although the exact pathways through which $A D$ develops or causes cognitive decline and dementia are still unknown, any knowledge on the complicated pathological cascade of $A D$ will help finding new treatments, prevention methods, or biomarkers for early diagnosis.

\section{Target population}

The main targets of the results of this thesis are researchers aiming to further investigate the underlying pathological processes responsible for $A D$. The results provide new targets for investigation, which will have to be researched in future studies, as most of the results are based on limited numbers of patients, using novel and experimental techniques. This thesis also contains new neuroimaging and analysis techniques which can be applied not only in $A D$, but also in other neurodegenerative diseases such as small vessel disease and multiple sclerosis. With the information provided in this thesis, improved techniques can be developed which are less burdening and more sensitive, to further study $A D$. 
The current results are also important for the general population, as anyone is at risk of developing $A D$. This thesis has provided further evidence for a vascular component of $A D$, but a cure or prevention method will require much more research. Still, if the role of vascular pathology in $A D$ receives more attention, awareness of the importance of vascular health at a stage before $A D$ symptoms arise will increase. A better vascular health for the general population will not only decrease the amount of vascular diseases, but may also help combat the symptoms of $A D$. An awareness campaign for the importance of vascular health to prevent dementia is likely to reach a large portion of the population, given the broad interest in $A D$. This is evident from the amount of attention $A D$ gets in the media. For example, when the results of Chapter 3 were published, the Radiological Society of North America issued a press release which was widely shared by international media outlets. This shows a global interest in $A D$, and in particular novel starting points, which can be capitalized upon.

\section{Innovation and future directions}

The innovative aspect of this thesis is that novel neuroimaging methods were applied to investigate microvascular pathology in $A D$. This required adaptation of existing methods and advanced analyses to increase sensitivity, as $A D$ is a progressive disease showing very subtle pathological changes in early stages. Given the results, the methods are proven to be feasible to detect group differences, but the neuroimaging methods applied in this thesis require improvement before clinical integration is possible and useful. Further application of the dual time resolution DCE-MRI sequence will elucidate the role of the blood-brain barrier in AD. Mainly, the results of the current thesis should be replicated in larger patient groups and in longitudinal studies to assess age-dependent effects. Also, the links between $A D$, cognitive decline, vascular pathology, and the blood-brain barrier should be further investigated. Furthermore, investigating the reproducibility of the DCE-MRI protocol is necessary to confirm that the method is reliable before clinical application can be considered.

The DCE-MRI protocol may also be useful to develop pharmaceuticals that target the brain. Because the blood-brain barrier very tightly regulates the passage of molecules from the blood into the brain, drugs that target the brain may also be stopped, which poses a challenge for the development of new drugs. Certain techniques may be used to temporarily open the blood-brain barrier to allow for the passage of drugs, such as the use of high-intensity focused ultrasound. DCE-MRI may be applied to validate these techniques and to measure their effectiveness. 
Improvements to the dual time resolution DCE-MRI sequence should include decreasing the scan time, increasing the spatial coverage of the fast portion of the protocol, decreasing the noise and imaging artefacts and improving the analysis to incorporate more physiological parameters to better model the in vivo conditions. Currently, the measured concentration differences and blood-brain barrier leakage rates are very small. As is shown in Chapter 4, improvements to the DCE-MRI sequence are both necessary and possible. If it is sufficiently improved and the reproducibility of the technique has been proven, DCE-MRI will be able to provide a truly quantitative measurement of blood-brain barrier leakage. The clinical application of the DCE-MRI protocol may include the ability to distinguish between a healthy and a non-healthy brain at risk for $A D$ on an individual basis. Other possible future uses of the DCE-MRI protocol may be to evaluate the effectiveness of treatments for the BBB, and to provide a general overview of cerebrovascular health to help monitor potential vascular risk factors. 


\section{Dankwoord}

Dit proefschrift is tot stand gekomen dankzij vele personen, die direct of indirect invloed hebben gehad op de inhoud van het proefschrift of op de schrijver ervan. Ik kan met zekerheid stellen dat deze invloeden overwegend positief waren, en daarom wil ik ook al die mensen bedanken voor hun bijdragen.

Allereerst wil ik mijn promotieteam bedanken. Beste prof. dr. ir. Walter Backes, prof. dr. Frans Verhey, dr. Jaap Jansen en dr. ir. Thijs van Osch, van harte bedankt voor jullie begeleiding, hulp en steun. Ik heb onze samenwerking altijd als zeer prettig ervaren, en jullie stonden altijd voor me klaar. Jullie open houding en feedback hebben tijdens mijn promotie een klimaat gecreëerd waarin ik me heb kunnen ontwikkelen en ik heb ontzettend veel geleerd.

Beste Walter, bedankt dat je zo veel tijd voor me hebt vrijgemaakt. De vele momenten dat je het hele bord volgeklad had met formules om een mathematische onderbouwing te geven zal ik me altijd herinneren. De dagelijkse begeleiding was altijd geweldig, en je talent om een tegenslag om te toveren in een positief punt hebben me door menig crisis heen geholpen.

Beste Jaap, ook jij stond altijd voor me klaar. Je inzicht en andere kijk op zaken waren bijzonder leerzaam, en samen met Walter zijn jullie een geweldig team. Ik heb me geen betere dagelijkse begeleiders kunnen voorstellen. Ontzettend bedankt voor alles!

Beste Saartje, je achtergrond in de psychologie en je open houding heb ik ontzettend gewaardeerd. De klinische kant van het verhaal raakt soms wat ondergesneeuwd bij de wat meer technisch georiënteerde mensen, terwijl dat misschien nu juist het belangrijkste is aan ons onderzoek. Je feedback op de artikelen was altijd bijzonder nuttig, en ik heb je dan ook ontzettend gemist nadat je begon aan je nieuwe baan.

Beste Frans, bedankt voor al je hulp en begeleiding. Je feedback en inzichten waren altijd bijzonder nuttig, en ik heb de samenwerking altijd erg goed gevonden. Bedankt dat je altijd voor me klaar stond!

Beste Thijs, bedankt voor de gezellige scansessies in Leiden, en bedankt voor je hulp met de scanprotocollen en analyses. Jouw perspectieven over het onderzoek waren altijd heel waardevol. 
Daarnaast wil ik graag alle coauteurs bedanken voor hun werk en ondersteuning. Jullie hulp was essentieel voor de totstandkoming van dit proefschrift. Ook wil ik de beoordelingscommissie, bestaande uit prof. dr. R.J. van Oostenbrugge, prof. dr. H.E. de Vries, dr. M.P.J. van Boxtel, dr. J.A.H.R. Claassen en natuurlijk prof. dr. J.E. Wildberger, van harte bedanken voor hun tijd en moeite, en voor het feit dat ze dit proefschrift positief beoordeeld hebben.

Voor al mijn collega's, zowel bij de Radiologie als bij de Neuropsychologie \& Psychiatrie, bedankt! Ik heb een ontzettend fijne tijd gehad tijdens mijn promotie, en dat is ook vooral tijdens de gezellige gesprekken tijdens het koffie drinken of vlaai eten. Ook zal ik de leuke feestjes van de Radiologie en de geweldige uitjes van de Neuropsychologie nooit meer vergeten. Marc en Jos, sorry voor het constant stuk maken van de radstations. Bedankt voor jullie geduld en de geweldige faciliteiten die jullie hebben gerealiseerd!

Dit proefschrift was niet mogelijk geweest zonder proefpersonen en hun begeleiders. Bedankt dat jullie de tijd en moeite wilden nemen om mee te helpen met dit onderzoek. Whitney, bedankt voor je steun. We hebben prachtige stukjes Nederland gezien op onze tandemfiets (en niet gezien toen het donker werd). Dankzij jouw navigatie hebben we het gered, en het was een geweldig avontuur!

Beste Ed en Heidi, dank voor jullie werk en steun. Ik heb veel gehad aan jullie input en ik heb onze samenwerking altijd bijzonder veel gewaardeerd.

Beste René, Frank, Tamar, May, Gerald, en Dorien, je kamergenoten zijn misschien wel de belangrijkste mensen tijdens een promotie, en ik had me geen betere kunnen wensen. Behalve natuurlijk wat de stand van de verwarming betreft, want dat bleef een strijd tot het bittere eind. Maar afgezien van dat heb ik altijd veel plezier gehad aan de discussies over promoveren, Marty plant, en andere willekeurige onderwerpen. Bedankt voor jullie steun en gezelschap, en ik wens jullie ontzettend veel succes voor de toekomst!

Paps en mams, jullie hebben me gemaakt tot wie ik vandaag ben. Zonder het doorzettingsvermogen dat ik van jullie heb geleerd, had ik het nooit gered. Daarom mogen jullie dan ook bijzonder trots zijn op dit resultaat. Bedankt!

Dag lief. Maastricht zit er op. Bedankt dat je me hebt gesteund, en bedankt dat je mee bent gegaan naar het zonnige zuiden. Ik weet niet of ik het gered had als je in Utrecht 164 
was blijven wonen. We hebben geweldige dingen meegemaakt, en onze bruiloft overtrof alle verwachtingen. $\mathrm{Nu}$ is het tijd voor een nieuw hoofdstuk. Laten we er wat moois van maken! Ik hou van je! 



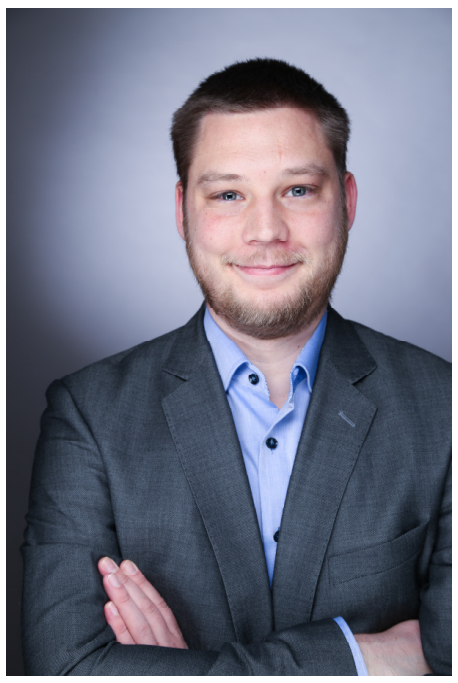

Harmen Jan van de Haar was born on the $21^{\text {st }}$ of January 1987 in Ede, the Netherlands, where he also graduated secondary school (VWO) at Het Streek in 2005. Due to his interest in the practical applications of technology in the hospital, he studied Technical Medicine at the University of Twente, in Enschede, the Netherlands, where he obtained his Bachelor of Science (BSC) in 2008 and his Master of Science (MSC) in 2012. During his Master's program, he did multiple internships in multiple institutions in the Netherlands, which were the University Medical Center Groningen, the Radboud University Medical Center in Nijmegen, and the Medical Center Amersfoort. After these internships, he started his master thesis, which was based on an internship from February 2011 to February 2012 at the Donders institute in Nijmegen. This internship was supervised by prof. dr. Norris, prof. dr. Buitelaar, and dr. Visser, and he researched tract analysis using diffusion weighted MRI for the investigation of brain white matter in autism. His master thesis sparked an interest in Neuroimaging, which is why he started his $\mathrm{PhD}$ project to research microvascular damage in Alzheimer's disease at the Maastricht University Medical Center at the department of Radiology, and the department of Neuropsychology \& Psychiatry, resulting in this PhD thesis. The project was performed under the direct supervision of prof. dr. ir. Backes, dr. Jansen and dr. Burgmans. He attended several national and international conferences and earned multiple awards for his research. His work on blood-brain barrier leakage in early Alzheimer's disease was met with much interest from the international press, resulting in multiple articles in newspapers and news websites. After finishing his $\mathrm{PhD}$, he started working as a software engineer for the international company Alten. 



\section{List of publications}

\section{Thesis}

HJ van de Haar, S Burgmans, PAM Hofman, FRJ Verhey, JFA Jansen, and WH Backes, "Blood-brain barrier impairment in dementia: Current and future in vivo assessments", Neuroscience and Biobehavioral Reviews, vol. 49, pp. 71-81, 2015.

HJ van de Haar, S Burgmans, JFA Jansen, MJP van Osch, MA van Buchem, M Muller, PAM Hofman, FRJ Verhey, and WH Backes, "Blood-Brain Barrier Leakage in Patients with Early Alzheimer Disease”, Radiology, 2016.

HJ van de Haar, JFA Jansen, CRLPN Jeukens, MJP van Osch, M A van Buchem, M Muller, PAM Hofman, FRJ Verhey, S Burgmans, and WH Backes, "Subtle blood-brain barrier leakage rate and spatial extent: considerations for dynamic contrast-enhanced MRI“, submitted

HJ van de Haar, JFA Jansen, MJP van Osch, MA van Buchem, M Muller, SM Wong, PAM Hofman, S Burgmans, FRJ Verhey, and WH Backes, "Neurovascular unit impairment in early Alzheimer's disease measured with magnetic resonance imaging”, Neurobiology of Aging, vol. 45, pp. 190-196, 2016.

HJ van de Haar, JFA Jansen, HIL Jacobs, S Burgmans, EHBM Gronenschild, RLH Handels, P Aalten, L Clerx, I Ramakers, FRJ Verhey, and WH Backes, "The effect of white matter hyperintensities on hippocampal structural connectivity in Alzheimer's disease", submitted.

\section{Other}

S Burgmans, HJ van de Haar, FRJ Verhey, and WH Backes, "Amyloid-beta interacts with blood-brain barrier function in dementia: A systematic review”, Journal of Alzheimer's Disease, vol. 35, no. 4. pp. 859-873, 2013.

JFA Jansen*, FCG van Bussel*, HJ van de Haar, MJP van Osch, PAM Hofman, MPJ van Boxtel, RJ van Oostenbrugge, MT Schram, CDA Stehouwer, JE Wildberger, and WH Backes, "Cerebral blood flow, blood supply, and cognition in Type 2 Diabetes Mellitus", Scientific Reports, 2016. 
CE Zhang*, SM Wong*, HJ van de Haar, J Staals, JFA Jansen, CRLPN Jeukens, PAM Hofman, RJ van Oostenbrugge, and WH Backes, "Blood-brain barrier leakage is more widespread in patients with cerebral small vessel disease", Neurology, 2017.

*: both authors contributed equally 